\title{
Atg21 functions during autophagy as a scaffold for the E3 ubiquitin-like complex in Atg8 lipidation
}

\author{
Dissertation \\ for the award of the degree \\ "Doctor rerum naturalium" \\ of the Georg-August-University Göttingen
}

within the doctoral program "Molecular Biology of Cells"

of the Georg-August-University School of Science (GAUSS)

submitted by

Lisa Angelika Juris

from Hamm (Westf.)

Göttingen, 2013 
Member of the Thesis Committee (First Reviewer):

Prof. Dr. Michael Thumm

Department of Molecular Cellbiology

Center for Biochemistry and Molecular Cell Biology

Georg-August-Universität, Göttingen

Member of the Thesis Committee (Second Reviewer):

Prof. Dr. Volker Lipka

Department of Plant Cell Biology

Schwann-Schleiden Centre

Georg-August-Universität, Göttingen

Member of the Thesis Committee:

Dr. Karin Kühnel

Department of Neurobiology

Max Planck Institute for Biophysical Chemistry, Göttingen

Date of oral examination: 05.02.2014 
Affidavit:

Herewith I declare, that I prepared this thesis on my own and with no other sources and aids than quoted.

Lisa Angelika Juris 


\section{Contents}

1 SUMMARY 1

2 INTRODUCTION 4

2.1 The yeast Saccharomyces cerevisiae as a model organism 4

2.2 Autophagy 5

2.2.1 Microautophagy 5

$\begin{array}{ll}\text { 2.2.2 Macroautophagy } & 6\end{array}$

$\begin{array}{lll}\text { 2.2.3 The Cytoplasm-to-Vacuole (Cvt) Pathway } & 7\end{array}$

$\begin{array}{lll}\text { 2.2.4 The pre-autophagosomal structure (PAS) } & 8\end{array}$

2.2.4.1 The Atg1 kinase complex 9

$\begin{array}{lll}\text { 2.2.4.2 The Atg9-containing vesicles } & 10\end{array}$

2.2.4.3 The Atg14-containing phosphoinositide 3-kinase complex 13

2.2.4.4 The Atg12- and Atg8-conjugating system 14

$\begin{array}{lll}\text { 2.2.4.5 The Atg2-Atg18 complex } & 17\end{array}$

3 MATERIALS AND METHODS

$\begin{array}{llr}3.1 & \text { Material } & 21\end{array}$

3.1.1 Saccharomyces cerevisiae strains $\quad 21$

3.1.2 Escherichia coli strains 23

3.1.3 Plasmids 23

3.1.4 Oligonucleotides 26

$\begin{array}{lll}3.1 .5 & \text { Media } & 30\end{array}$

3.1.5.1 YPD-medium, pH $5.5 \quad 30$

3.1.5.2 CM-medium, pH $5.6 \quad 30$

$\begin{array}{lll}\text { 3.1.5.3 SD(-N)-medium } & 31\end{array}$

3.1.5.4 LB-medium, $\mathrm{pH} 7.5 \quad 31$

3.1.5.5 SOC-medium, $\mathrm{pH} 7.5 \quad 31$

3.1.6 Antibodies 31

3.1.7 Commercial available Kits 32

3.1.8 Chemicals and consumables 32

$\begin{array}{lll}3.1 .9 & \text { Equipment } & 34\end{array}$

3.2 Cultivation of $S$. cerevisiae

3.2.1 Growth of liquid yeast cultures 35 
$\begin{array}{lll}\text { 3.2.2 } & \text { Short-term storage } & 35\end{array}$

3.2.3 Long-term storage 36

3.2.4 Cell density determination 36

$\begin{array}{lll}3.2 .5 & \text { Split-ubiquitin assay } & 36\end{array}$

3.3 Cultivation of $E$. coli cultures $\quad 36$

3.3.1 Growth of liquid E. coli cultures 36

$\begin{array}{lll}3.3 .2 & \text { E. coli short-term storing } & 36\end{array}$

$\begin{array}{lll}\text { 3.3.3 } & \text { E. coli long-term storing } & 37\end{array}$

3.3.4 Preparation of electrocompetent E. coli cells 37

$\begin{array}{llr}3.4 & \text { Microscopy } & 37\end{array}$

$\begin{array}{lll}3.4 .1 & \text { Fluorescence microscopy } & 37\end{array}$

3.4.2 Bimolecular Fluorescence Complementation (BiFC) 38

$\begin{array}{lll}\text { 3.4.3 Determination of colocalization rates } & 39\end{array}$

$\begin{array}{lll}3.5 & \text { Molecular biology methods } & 39\end{array}$

$\begin{array}{lll}\text { 3.5.1 Isolation of chromosomal DNA } & 39\end{array}$

3.5.2 High efficiency transformation of DNA in yeast 40

3.5.3 "Quick and Dirty" transformation of plasmid-DNA in yeast 40

3.5.4 Transformation of plasmid-DNA into E. coli 41

3.5.5 Plasmid-DNA isolation from E. coli 41

3.5.6 DNA agarose gel electrophoresis 41

3.5.7 DNA gel extraction (Gel Extraction Kit) 42

$\begin{array}{lll}3.5 .8 & \text { Polymerase chain reaction } & 42\end{array}$

3.5.9 Molecular cloning $\quad 42$

3.5.10 Plasmid construction $\quad 43$

3.5.10.1 Plasmid constructs used in split-ubiquitin assay 43

3.5.10.2 Plasmid constructs for BiFC 44

3.5.10.3 Atg16 plasmid constructs $\quad 45$

3.5.11 Site-directed mutagenesis of plasmids $\quad 46$

3.5.12 Sequencing of DNA 46

3.5.13 Gene deletion using homologous recombination 46

\section{$\begin{array}{llr}3.6 & \text { Biochemical methods } & 47\end{array}$}

$\begin{array}{lll}\text { 3.6.1 SDS-Polyacrylamide-Gel-Electrophorese (SDS-PAGE) } & 47\end{array}$

$\begin{array}{lll}3.6 .2 & \text { Western Blot analysis } & 48\end{array}$

$\begin{array}{lll}\text { 3.6.3 Colloidal Coomassie staining } & 49\end{array}$

3.6.4 Determination of protein concentrations $\quad 50$

$\begin{array}{ll}3.6 .5 & \text { Alkaline lysis of yeast cells } \\ & 50\end{array}$

3.6.6 Cell lysis for detection of Atg8-PE $\quad 50$ 
3.6.7 Preparation of whole cell extracts - the mild cell lysis procedure 51

$\begin{array}{lll}3.6 .8 & \text { GFP-TRAP } & \\ & & 51\end{array}$

3.6.9 Gelfiltration chromatography 52

3.6.10 Density gradient centrifugation 53

3.6.11 Pull down of His-SUMO-Atg21 53

3.6.11.1 Purification of recombinant 6xHis-SUMO-Atg21 and 6xHis-SUMO 53

3.6.11.2 6xHis-SUMO-Atg21 pull down with crude yeast cell extract 54

3.6.11.3 Purification of GST-Atg16

3.6.11.4 6xHis-SUMO-Atg21 pull down with recombinant proteins 55

3.6.12 Mass spectrometry analysis 56

3.6.12.1 Materials $\quad 56$

3.6.12.2 In-gel digestion $\quad 56$

3.6.12.3 Peptide extraction $\quad 56$

3.6.12.4 Mass spectrometric analysis $\quad 57$

$\begin{array}{lll}\text { 3.6.12.5 Database Searching } & 57\end{array}$

$\begin{array}{llr}4 & \text { RESULTS } & 58\end{array}$

$\begin{array}{llr}4.1 & \text { Aim of the study } & 58\end{array}$

$\begin{array}{llr}4.2 & \text { Localization studies of Atg21 } & 58\end{array}$

4.2.1 Part of Atg21-YFP was detected at the PAS 58

4.2.2 Atg21 is not transported into the vacuole 63

4.3 Identification of interaction partner of Atg21 65

4.3.1 Atg21 is part of a high molecular weight complex 65

4.3.1.1 Gelfiltration analysis of Atg21 65

4.3.1.2 Sorbitol density gradient centrifugation 69

4.3.2 Pull down und mass spectrometry analysis to identify potential Atg21 interaction partner $\quad 72$

4.3.3 Split-ubiquitin analysis of Atg21

4.3.4 Interaction studies of Atg21 und At27 76

4.3.4.1 Atg27 and Atg21 interact with each other in split-ubiquitin assay $\quad 77$

4.3.4.2 Atg21 and Atg27 colocalized in direct fluorescence microscopy 80

4.3.4.3 Analysis of the potential interaction of Atg21 and Atg27 by pull down and GFP-

TRAP ${ }^{\circledR} \quad 82$

4.3.5 Interaction studies of Atg21 und Atg8 84

4.3.5.1 Bimolecular Fluorescence Complementation (BiFC) analysis 85

4.3.5.2 Is Atg8 part of the Atg21 high molecular weight complex detected in gelfiltration? 
4.3.6 Interaction studies of Atg21 und Atg16 94

4.3.6.1 Atg21 and Atg16 interact with each other in the split-ubiquitin assay 94

4.3.6.2 Fluorescence microscopy analysis of Atg5 and Atg16 96

4.3.6.3 Interaction studies of Atg16 and Atg21 using pull down and GFP-TRAP ${ }^{\circledR}$ analysis

100

4.3.6.4 Determination of the Atg21- interacting domain of Atg16 102

5 DISCUSSION 111

$\begin{array}{llr}5.1 & \text { Part of Atg21 localizes to the PAS } & 112\end{array}$

$\begin{array}{rrr}5.2 & \text { Atg21 is located on the outer autophagosomal membrane } & 118\end{array}$

$\begin{array}{lll}5.3 & \text { Atg21 is part of a high molecular weight complex } & 120\end{array}$

$\begin{array}{lll}5.4 & \text { Identification of Atg21 interaction partner } & 122\end{array}$

$\begin{array}{lll}5.5 & \text { Interaction studies of Atg21 and Atg27 } & 124\end{array}$

\begin{tabular}{|rr}
5.6 & Interaction studies of Atg21 and Atg8
\end{tabular}

$\begin{array}{lll}5.7 & \text { Interaction studies of Atg21 and Atg16 } & 130\end{array}$

6 BIBLIOGRAPHY 141

7 ACKNOWLEDGEMENT 151

8 CURRICULUM VITAE ERROR! BOOKMARK NOT DEFINED. 


\section{List of Figures}

$\begin{array}{lr}\text { Figure 1: Life cycle of Saccharomyces cerevisiae } & 4\end{array}$

$\begin{array}{ll}\text { Figure 2: Scheme of macroautophagy and the Cvt-pathway } & 7\end{array}$

$\begin{array}{ll}\text { Figure 3: Scheme of the Atg1 kinase complex } & 10\end{array}$

Figure 4: Atg9-containing vesicles contribute in membrane dynamics in autophagy 11

Figure 5: Model of the Atg23-Atg9-Atg27 complex 12

Figure 6: Vps34 is present in two complexes required for different cellular processes 13

Figure 7: The two ubiquitin-like protein conjugation machineries in autophagy $\quad 14$

$\begin{array}{lr}\text { Figure 8: Structure of Kluyveromyces lactis Hsv2 } & 18\end{array}$

$\begin{array}{lr}\text { Figure 9: Scheme of semi dry Western Blot setting } & 48\end{array}$

Figure 10: Scheme of Wet Western Blot settings $\quad 49$

Figure 11: Part of Atg21 is localized at the PAS $\quad 60$

Figure 12: Additional analysis of the PAS localization of Atg21-YFP 61

Figure 13: Complementation study of mCherry-Atg21 by monitoring ApeI maturation and determination of its PAS rate.

Figure 14: mCherry-Atg21 is not transported into the vacuole 64

Figure 15: Atg21-TAP was present in a sensitive high molecular weight complex in $\begin{array}{ll}\text { gelfiltration } & 66\end{array}$

Figure 16: Alternative mild cell lysis procedure keeps the sensitive high molecular weight $\begin{array}{ll}\text { Atg21 complex intact } & 68\end{array}$

Figure 17: The high molecular weight Atg21 complex could not be detected after glycerol $\begin{array}{ll}\text { density gradient centrifugation } & 69\end{array}$

Figure 18: mCherry-Atg21 is present as a high molecular weight complex in sorbitol $\begin{array}{ll}\text { density gradient centrifugation } & 71\end{array}$

$\begin{array}{ll}\text { Figure 19: Pull down analysis of SUMO-Atg21 } & 73\end{array}$

Figure 20: Scheme of the principle of the split-ubiquitin assay 75

Figure 21: An interaction of Atg27 and Atg21 in split-ubiquitin assay was detected 77

Figure 22: Interaction analysis of truncated Atg27 in split-ubiquitin assay $\quad 79$

Figure 23: mCherry-Atg21 and Atg27-GFP colocalized in direct fluorescence microscopy 81

Figure 24: Atg27 does not specifically interact with Atg21 in pull down and GFP-TRAP ${ }^{\circledR}$ $\begin{array}{ll}\text { experiments } & 83\end{array}$

Figure 25: Atg21 and Atg8 interact with each other in split-ubiquitin assay 85

Figure 26: Bimolecular Fluorescence Complementation (BiFC) analysis of Atg8 and Atg21

\begin{tabular}{ll} 
Figure 27: BiFC study of MET25-Atg21 C-YC and ADH-N-YN Atg8 & 87 \\
\hline
\end{tabular}

Figure 28: BiFC study of MET25 Atg21 C-YC and MET25 N-YN Atg8 91

Figure 29: Atg8 distribution in gelfiltration was not changed in the absence of Atg21 93

Figure 30: Atg16 and Atg21 interact in the split-ubiquitin assay 95

Figure 31: Atg5 and Atg16 are absent from the PAS in growing atg8 $\operatorname{atg} 21 \Delta$ cells 97 
Figure 32: Atg16 and Atg21 colocalize in the absence of Atg5

Figure 33: Interaction studies of Atg16 and Atg21 using GFP-TRAP ${ }^{\circledR}$ and pull down experiments

Figure 34: Atg21 interacts with the coiled-coil domain of Atg16

Figure 35: Fluorescence microscopic analysis of the Atg16-GFP point mutants

Figure 36: Amino acids D101 and E102 of Atg16 mediate the interaction with Atg21

\section{List of Tables}

Table 1: Yeast strains used in this study

Table 2: E. coli strains used in this study

Table 3: Plasmids used in this study

Table 4: Oligonucleotides used in this study

Table 5: Antibodies used in this study

Table 6: Commercial available Kits used in this study

Table 7: Special chemicals

Table 8: Equipment used in this study

Table 9: Overview of the used filter sets in fluorescence microscopy

Table 10: Contents of the separating gel

Table 11: Contents of the stacking gel

Table 12: Densities of the glycerol or sorbitol solutions used in density gradient centrifugation

\section{List of Equations}

Equation 1: Equation to calculate optimal plasmid-insert ratio in ligation mix 


\begin{tabular}{|c|c|}
\hline Abbreviation & \\
\hline- & without \\
\hline${ }^{\circ} \mathrm{C}$ & Degree in Celsius \\
\hline A & Ampere \\
\hline aа & Amino acid(s) \\
\hline ade & Adenine \\
\hline $\mathrm{ADH}$ & Alcohol dehydrogenase \\
\hline AIM & Atg8-interacting motif \\
\hline Arg & Arginine \\
\hline ApeI & Aminopeptidase I \\
\hline APS & Ammonium persulfate \\
\hline Atg & Autophagy-related protein \\
\hline $\mathrm{BiFC}$ & Bimolecular Fluorescence Complementation \\
\hline $\operatorname{ccd}$ & coiled-coil domain \\
\hline CM medium & Complete minimal medium \\
\hline CPY & Carboxypeptidase C \\
\hline Cub & C-terminal part of ubiquitin \\
\hline Cvt & Cytoplasm-to-Vacuole \\
\hline DNA & Desoxyribonucleic acid \\
\hline DTT & Dithiothreitol \\
\hline E. coli & Escherichia coli \\
\hline EDTA & Ethylenediamintetraacetate-disodium salt \\
\hline eYFP & enhanced YFP \\
\hline FOA & 5-Fluoroorotic Acid \\
\hline g & gram \\
\hline g & Gravity \\
\hline GFP & Green fluorescent protein \\
\hline Gly & Glycine \\
\hline GST & Glutathione-S-transferase \\
\hline $\mathrm{h}$ & hour \\
\hline HA & Human influenza hemagglutinin \\
\hline His & Histidine \\
\hline HRPO & Horsereddish peroxidase \\
\hline
\end{tabular}




\begin{tabular}{|c|c|}
\hline IPTG & Isopropyl- $\alpha$-D-thiogalactopyranoside \\
\hline KAN & Kanamycin \\
\hline $\mathrm{kDa}$ & kilodalton \\
\hline 1 & liter \\
\hline LB & Lysogeny broth \\
\hline Leu & Leucine \\
\hline LIR & LC3-interacting region \\
\hline Lys & Lysine \\
\hline M & molar \\
\hline $\mathrm{m}$ & mature \\
\hline $\mathrm{m}$ & meter \\
\hline $\mathrm{m}$ & milli \\
\hline mCherry & monomeric Cherry \\
\hline met & Methionine \\
\hline $\min$ & minute \\
\hline $\mathrm{N}$ & Nitrogen \\
\hline $\mathrm{n}$ & nano \\
\hline NatNT2 & Nourseotricine \\
\hline Nui & $\mathrm{N}$-terminal part of ubiquitin \\
\hline $\mathrm{OD}_{600}$ & Optical density (600 nm) \\
\hline PAS & Pre-autophagosomal structure \\
\hline PE & Phosphatidylethanolamine \\
\hline PGK & Phosphoglycerate kinase \\
\hline $\mathrm{pH}$ & negative logarithm of $\mathrm{H}^{+}$concentration \\
\hline Phe & Phenylalanine \\
\hline PI3P & Phosphatidylinositol 3-phosphate \\
\hline $\mathrm{PI}(3,5) \mathrm{P}_{2}$ & Phosphatidylinositol 3,5-bisphosphate \\
\hline PMN & Piecemeal Microautophagy of the Nucleus \\
\hline PMSF & Phenylmethylsulfonylfluoride \\
\hline $\mathrm{pr}$ & Precursor \\
\hline PROPPIN & $\beta$-propeller that binds polyphosphoinositides \\
\hline RFP & Red fluorescent protein \\
\hline rpm & round per minute \\
\hline
\end{tabular}


RT

S

S. cerevisiae

SD

SDS

TAP

TCA

TORC1

$\operatorname{Trp}$

Ura

$\mathrm{v} / \mathrm{v}$

$\mathrm{V}$

$\mathrm{w} / \mathrm{v}$

WT

YC

YFP

YN

YPD

$\mu$

$\beta-\mathrm{ME}$
Room temperature

second

Saccharomyces cerevisiae

Synthetic defined

Sodium dodecyl sulfate

Tandem Affinity Purification

Trichloroacetic acid

Target of Rapamycin Complex 1

Tryptophan

Uracile

volume per volume

volt

weight per volume

Wild type

C-terminal part of eYFP

Yellow fluorescent protein

$\mathrm{N}$-terminal part of eYFP

Yeast peptone dextrose

micro

Beta mercaptoethanol 


\section{Summary}

Autophagy summarizes a family of intracellular lysosomal (vacuolar) degradative transport process. It is used for the removal and recycling of cytoplasm, protein aggregates and organelles to maintain cellular homeostasis. During macroautophagy in yeast, the double membrane layered transport vesicles (autophagosomes) are formed at the pre-autophagosomal structure (PAS). They finally fuse with the vacuole for degradation and recycling of their contents. Beside this unselective degradation of bulk cytoplasm including organelles, there are several selective types of autophagy. In yeast, the Cytoplasm-to-vacuole (Cvt) pathway selectively transports the hydrolases $\alpha$ mannosidase, proaminopeptidase I and aspartyl aminopeptidase to the vacuole under nutrient rich conditions.

A hallmark of autophagy is the use of two ubiquitin-like conjugation systems. One covalently couples the ubiquitin-like Atg12 to Atg5. The resulting Atg12Atg5 conjugate forms a complex with Atg16 that acts as an E3-like enzyme in the second ubiquitin-like conjugation reaction, which couples Atg8 to the head group of the membrane lipid phosphatidylethanolamine (PE). Atg16 specifies the lipidation site, but how it selectively recognizes and binds to the PI3P-positive PAS remained unclear.

In this study, the localization and function of Atg21 was investigated using the model organism Saccharomyces cerevisiae. Atg21 forms a WD40 propeller that is peripherally membrane associated by binding to $\mathrm{PI} 3 \mathrm{P}$ and $\mathrm{PI}(3,5) \mathrm{P}_{2}$. So far, Atg21 has only been detected at endosomal compartments and at vertices of vacuolar junctions. Atg21 is required for selective types of autophagy as the Cvt pathway and efficient bulk macroautophagy. In the absence of Atg21 no Cvt vesicles are formed, suggesting a fundamental role for Atg21 in the PAS assembly especially during selective types of autophagy. Indeed, growing atg21 4 cells fail to efficiently lipidate Atg8 and to recruit Atg8 and Atg5 to the PAS. Since Atg21 has not been detected at the PAS so far, its precise molecular function during autophagy remained elusive. 
Using direct fluorescence microscopy, this study revealed, for the first time, that part of Atg21 is also present at the PAS. The presence of Atg21 at the PAS is dependent on Atg14 and therefore on the PAS-specific PI3P pool. Moreover, it could be shown in this study, that Atg21 recruits the Atg12-Atg5/Atg16 complex to the PAS via direct interaction with Atg16. Two conserved surface residues within the coiled-coil domain of Atg16 (D101 and E102) were identified to specifically bind Atg21. Thereby the underlying mechanism of Atg16-mediated recruitment of the Atg12-Atg5/Atg16 complex to autophagosomal membranes has been elucidated in this study. More importantly, it was shown, that the interaction of Atg21 and Atg16 was not only a prerequisite for the proper PAS localization of the Atg12-Atg5/Atg16 complex but also for its autophagic function. Consequently, interaction of Atg16 and Atg21 is needed for efficient Atg8 lipidation.

Importantly, this study also revealed that Atg21 and Atg8 probably interact with each other. This interaction occurred with unlipidated Atg8, suggesting, that Atg21 not only mediates the membrane association of the E3 enzyme Atg12Atg5/Atg16, but also acts as a scaffold for Atg8 lipidation reaction. Therefore, Atg21 represents the missing link that mediates PI3P-dependent PAS association of the lipidation machinery by recruiting the activated substrate Atg8 and the E3 enzyme required for the transfer of Atg8 to PE. Atg21 thus represents a key factor to specify the lipidation site. This model provides a reasonable explanation for the elementary function of Atg21 in Cvt vesicle formation.

By using gelfiltration chromatography and sorbitol gradient density centrifugation Atg21 was identified as part of a high molecular weight complex. This Atg21 complex is very sensitive to the cell lysis procedure and buffer conditions. Therefore, a mild cell lysis procedure was established to keep the sensible complex intact as a prerequisite not only for further analysis of the complex but also for the identification and verification of additional potential Atg21 interacting proteins.

Pull down experiments and following mass spectrometry analysis identified the target of rapamycin complex I (TORC1) as potential interaction partner of Atg21. 
It is already known, that the kinase activity of TORC1 negatively regulates autophagy.

A specific interaction of Atg21 with the type I membrane protein Atg27 but with no other member of the trimeric Atg27-Atg9-Atg23 complex was detected using the split-ubiquitin system. Moreover, it could be suggested that Atg21 interacted with the cytosol-facing C-terminal part of Atg27. In addition, using direct fluorescent microscopy it could be shown here that a considerable part of Atg21 colocalized with Atg27, supporting a potential interaction. The relevance of this putative interaction will be topic of further studies. 


\section{Introduction}

\subsection{The yeast Saccharomyces cerevisiae as a model organism}

The eukaryotic organism Saccharomyces cerevisiae belongs to the family of the budding yeasts. It is a unicellular organism and has a round to oval shape with 5 to $10 \mu \mathrm{m}$ in diameter. S. cerevisiae has a simple life cycle (Figure 1). It can exist in a either haploid or diploid form. Both forms grow by mitosis and budding and are able to double in about $100 \mathrm{~min}$, when exposed to sufficient nutrients. Under nutrient-deficient conditions, diploid cells undergo meiosis to produce an ascus containing four haploid spores. After a change in nutrient conditions, the spores can grow as haploid cells. Two haploid cells with different mating type (a and $\alpha$ ) can mate to form a diploid cell.

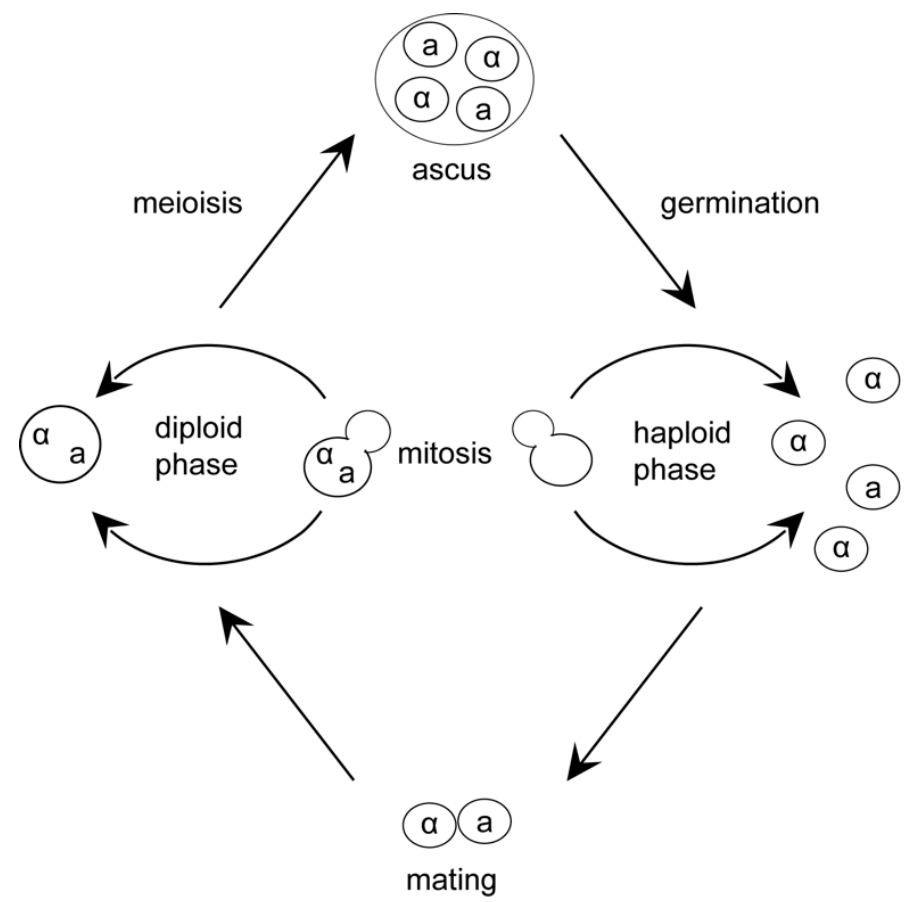

Figure 1: Life cycle of Saccharomyces cerevisiae

Using S. cerevisiae as a model organism, it can be grown on defined media to control its chemical and physical environment. The S. cerevisiae genome is fully sequenced. A haploid cell harbors a set of 16 chromosomes coding for about 6000 genes (Goffeau et al., 1996). In the last decades, efficient techniques have been developed, that easily enable a modification or deletion of a target allele 
with absolute precision (Goffeau et al., 1996; Janke et al., 2004; Knop et al., 1999; Longtine et al., 1998; Wach et al., 1997; Wach et al., 1994). Most of the proteins in S. cerevisiae are highly conserved and information obtained in yeast can often be transferred to higher eukaryotes making the baker's yeast $S$. cerevisiae to an ideal and favored model organism in genetic and biochemical research.

\subsection{Autophagy}

Autophagy describes a specific transport mechanism to the degradative organelle within a cell. It is highly conserved among all eukaryotes and primarily protects cells under stress conditions. Under starvation conditions, the unspecific degradation of cytoplasm and organelles produces free amino and fatty acids, which are subsequently used for the synthesis of new proteins or as energy source. In addition to cellular homeostasis, autophagy plays a crucial role in several physiological processes as autophagic programmed cell death, organelle homeostasis, developmental processes, ageing, immunity and defense against microbial invasion (Chen and Klionsky, 2011). Autophagy is also involved in several human pathophysiologies such as cancer, myopathies, neurodegeneration, heart and liver diseases and gastrointestinal disorders (Choi et al., 2013). In yeast, autophagy can be divided into two major groups: microautophagy and macroautophagy.

\subsubsection{Microautophagy}

During microautophagy a cargo is directly engulfed by the vacuolar membrane. In S. cerevisiae, micronucleophagy or piecemeal microautophagy of the nucleus (PMN) is one specific variant of microautophagy (Krick et al., 2008b). During PMN non-essential portions of the nucleus are directly sequestrated and degraded by the vacuole. PMN occurs at nucleus-vacuole (NV) junctions, which are generated by the interaction of the vacuolar membrane protein Vac8 and the outer nuclear membrane protein Nvj1 (Pan et al., 2000; Roberts et al., 2003). Nvj1 additionally recruits Tsc13 and Osh1, both involved in lipid metabolism, to the NV junctions (Kvam and Goldfarb, 2007). Upon starvation, the NV junctions bud into invaginations at the vacuolar membrane to create a PMN vesicle. This PMN vesicle is released into the vacuolar lumen after fusion of the nuclear and the vacuole membrane and finally degraded by vacuolar hydrolases (Krick et al., 
2008b). Three membranes limit a PMN vesicle; the two inner membranes originate from the nuclear envelope, the outermost membrane from the vacuole membrane (Krick et al., 2008b). PMN requires both, core components of the macroautophagic machinery and components of the selective autophagic machinery, defining PMN as a novel variant of selective microautophagy (Krick et al., 2008b; Roberts et al., 2003). Interestingly, PMN does not require the homotypic vacuole fusion machinery, suggesting a crucial role for the autophagic machinery in the terminal engulfment and fusion stages of PMN (Krick et al., 2008b).

\subsubsection{Macroautophagy}

In contrast to microautophagy, macroautophagy is characterized by the formation of double-membrane layered transport vesicles at the preautophagosomal structure (PAS). In yeast, the formation of the transport vesicles starts with a cup-shaped membrane, the phagophore, which expands and finally closes to form a double-membrane layered transport vesicle, named autophagosome (Yorimitsu and Klionsky, 2005) (Figure 2). The origin of the membranes for autophagosome biogenesis is still under debate and there are several organelles discussed as possible sources such as the plasma membrane, the endoplasmatic reticulum, the Golgi apparatus, endosomes or mitochondria (Lynch-Day et al., 2010; Mari et al., 2010; Taylor et al., 2012; Yamamoto et al., 2012). Due to the extensive need of membranes during autophagy, most probably different rather than one single organelle might serve as a membrane source.

The outer membrane of the autophagosome fuses with the vacuolar membrane to release a still one membrane-layered vesicle, the autophagic body, into the vacuole (Baba et al., 1994). This fusion event depends on the homotypic vacuolar fusion machinery (Ishihara et al., 2001). Within the vacuole, the membrane of the autophagic body is lysed and the cargoes are subsequently degraded or processed by vacuolar hydrolases. Under normal growth conditions macroautophagy acts as constitutive process at a low level, but it is strongly upregulated under stress conditions as nutrient or energy starvation (Parzych and Klionsky, 2013; Yorimitsu and Klionsky, 2005). 


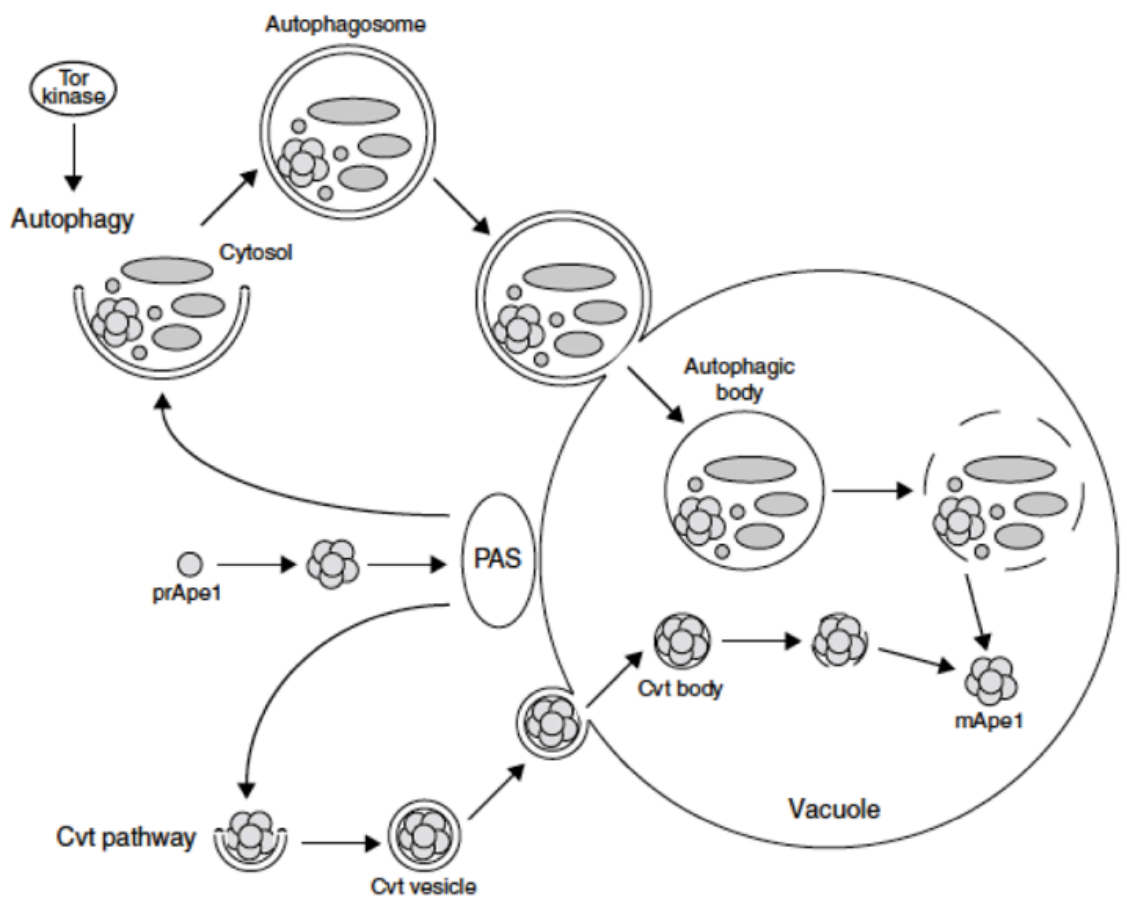

Figure 2: Scheme of macroautophagy and the Cvt-pathway (Yorimitsu and Klionsky, 2005)

The term macroautophagy is often used for the unselective degradation of bulk cytoplasm including organelles. There are also several selective variants of autophagy for the specific degradation of superfluous or damaged organelles as mitochondria (mitophagy), peroxisomes (pexophagy), endoplasmatic reticulum (reticulophagy) and ribosomes (ribophagy) (He and Klionsky, 2009). In general, selective macroautophagy is characterized by the use of a receptor protein, which links the cargo to the autophagic machinery (Mijaljica et al., 2012). In addition, the transport vesicles are specifically formed around a particular cargo und exclude therefore cytoplasm or unspecific components (Reggiori and Klionsky, 2013).

\subsubsection{The Cytoplasm-to-Vacuole (Cvt) Pathway}

In yeast, the Cytoplasm-to-Vacuole (Cvt) pathway displays another selective variant of autophagy and represents the constitutive transport of the hydrolases $\alpha$-mannosidase I (AmsI), the aspartyl aminopeptidase (Ape4) and aminopeptidase I (ApeI) to the vacuole (Harding et al., 1995; Hutchins and Klionsky, 2001; Yuga et al., 2011). ApeI is synthesized as a precursor form with a N-terminal propeptide (prApeI) in the cytosol, where it forms a dodecameric complex. The propeptide mediates the formation of prApeI dodecamers into a 
large oligomeric structure (ApeI-complex) and also binds to the cargo receptor Atg19, which links the ApeI-complex to the autophagic machinery at the PAS (Chang and Huang, 2007; Morales Quinones et al., 2012; Scott et al., 2001; Shintani and Klionsky, 2004; Yorimitsu and Klionsky, 2005). In addition, Atg19 binds to the additional cargoes AmsI and Ape4, allowing them to use the ApeI transport system (Shintani et al., 2002; Yuga et al., 2011). prApeI, Atg19, AmsI and Ape4 are packed into a sequestering vesicle called Cvt vesicle, that is significantly smaller in size compared to autophagosomes and exclude cytoplasm. The Cvt vesicle is transported to the vacuole, where prApeI is processed by cleavage of its propeptide to generate the matured and active form of ApeI (mApeI) (Klionsky et al., 1992; Morales Quinones et al., 2012; Scott et al., 1997; Scott et al., 1996; Shintani et al., 2002; Suzuki, 2012) (Figure 2). mApeI still forms dodecamers, but due to the lack of the propeptide, does not form the ApeI complex in the vacuole (Morales Quinones et al., 2012). The Cvt pathway is a constitutive transport mechanism and takes place under growing conditions (Scott et al., 1997). As a selective form of autophagy, it relies on the same core autophagy related proteins like macroautophagy and additionally requires some specific proteins. Therefore, information obtained in selective types of autophagy, can often be transferred to other autophagic pathways (Lynch-Day and Klionsky, 2010; Morales Quinones et al., 2012).

\subsubsection{The pre-autophagosomal structure (PAS)}

The pre-autophagosomal structure (PAS) is defined as that site within a cell, where the formation of the autophagosomes and Cvt vesicles is initiated (Suzuki et al., 2001; Suzuki et al., 2007). Yeast cells usually have one PAS, located near the vacuole under both normal and starvation conditions. Most of the autophagyrelated proteins, termed Atg, are at least partially localized to the PAS (Klionsky et al., 2003; Suzuki et al., 2007) In yeast, more than 30 Atgs are known. Among them, 18 proteins belong to the core autophagic machinery, characterizing those proteins required for both macroautophagy and its selective variants (Araki et al., 2013; Motley et al., 2012; Suzuki, 2012; Suzuki and Ohsumi, 2010). These proteins are recruited to the PAS in a specific hierarchy (Suzuki et al., 2007) and are commonly classified into six functional groups: I) the Atg1 kinase complex 
II) the Atg9-containing vesicles III) the Atg14-containing phosphoinositides 3kinase complex IV) the Atg12-conjugating system V) the Atg8-conjugating system and VI) the Atg2-Atg18 complex.

\subsubsection{The Atg1 kinase complex}

The Atg1 kinase complex serves as a basic scaffold structure for the PAS assembly and is required for the recruitment of further autophagy-related proteins. Atg1 is the solely serine/threonine kinase involved in autophagy (Matsuura et al., 1997). Besides Atg1 itself, no other target for the kinase has been identified so far. Atg1 directly interacts with its regulator Atg13, what enhances its kinase activity (Kamada et al., 2000). Atg1 and Atg13 probably exhibit a core regulator function at an early step in autophagy induction (Reggiori and Klionsky, 2013). The Atg13-Atg1 complex itself is regulated by nutrients. Under nutrient-rich conditions, Atg13 and Atg1 are highly hyperphosphorylated (Scott et al., 2000). The phosphorylation is partly mediated by the nutrient sensors Target of rapamycin complex 1 (TORC1) and/or protein kinase A (PKA) (Budovskaya et al., 2005; Kamada et al., 2010; Yorimitsu et al., 2009). The kinase TORC1 acts as a negative regulator of autophagy. It is active under nutrient-rich conditions and inhibits autophagy. Upon starvation it is inactivated and autophagy is induced. Since TORC regulates Atg13 phosphorylation, Atg13 becomes rapidly dephosphorylated upon autophagy induction (Loewith and Hall, 2011; Scott et al., 2000). For yeast, it was originally reported that the phosphorylation of Atg13 leads to a reduced Atg1 binding affinity, preventing the Atg1 kinase activity under nutrient-rich conditions (Kamada et al., 2000). However, recent studies revealed, that Atg1 and Atg13 might interact constitutively with each other, independent of the nutrient conditions (Kraft et al., 2012). This model would fit nicely with results from higher eukaryotes and indicates that the phosphorylation of yeast Atg1 and most likely Atg13 rather than their interaction is essential for Atg1 activity (Kraft et al., 2012). In general, Atg1 has a dual role in autophagy. Atg1 mutants defective in Atg13 binding, fail to efficiently assemble the PAS under starvation conditions, suggesting a scaffold function for Atg1 at the PAS. In contrast, Atg1 kinase mutants assemble the PAS but are defective in Atg protein dissociation 
kinetics (Cheong et al., 2008). Thus, the Atg1 kinase activity is probably involved in later steps of autophagy.

In addition to Atg1 and Atg13, the Atg1 kinase complex includes the ternary Atg17-Atg31-Atg29 complex and also Atg11, Atg20 and Atg24 (Figure 3).

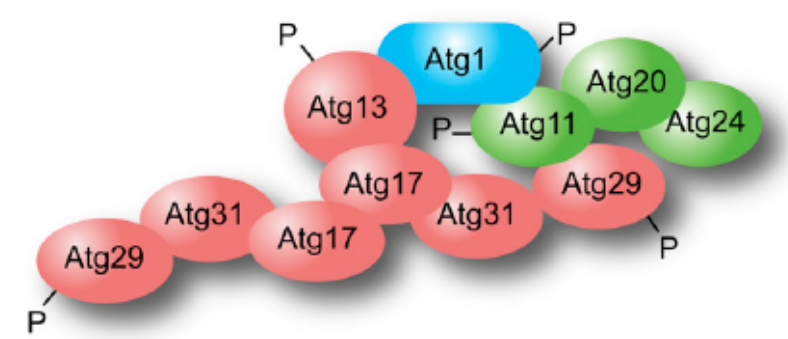

Figure 3: Scheme of the Atg1 kinase complex (Reggiori and Klionsky, 2013)

So far, it is not known, if all of these proteins are present in the complex at the same time or if there are subcomplexes that vary in their composition depending on nutrient conditions (Inoue and Klionsky, 2010). Atg17 acts as a central component in PAS assembly under autophagy-inducing conditions (Cheong et al., 2008; Suzuki et al., 2007). It forms a constitutive complex with Atg29 and Atg31 (Kabeya et al., 2009; Kawamata et al., 2008). In addition, self-interaction of Atg17 leads to a stable dimeric Atg17-Atg29-Atg31 complex, which is probably recruited to the PAS under both, nutrient-rich condition and starvation (Chew et al., 2013; Ragusa et al., 2012). Atg17 also enhances the Atg1 kinase activity by an unknown mechanism (Kamada et al., 2000). Atg17 is essential for PAS assembly during macroautophagy, but not for selective types of autophagy. For selective types of autophagy, Atg11 adopts the function of Atg17 and acts as a scaffold protein that directs further Atg proteins to the PAS (Suzuki et al., 2007). However, though involved in the formation of the starvation-specific PAS, Atg11 is not required for bulk autophagy. Atg11 also serves as an adaptor for selective autophagic cargoes at the PAS by binding receptor proteins involved in selective autophagy such as Atg19 (Cvt pathway) and Atg32 (mitophagy) (Mijaljica et al., 2012).

\subsubsection{The Atg9-containing vesicles}

Among the core autophagy machinery, Atg9 is the sole integral membrane protein. It contains six membrane-spanning domains, its $\mathrm{C}$ - and $\mathrm{N}$-terminus 
facing the cytosol (Legakis et al., 2007). Atg9 is one of the first proteins recruited to the PAS (Suzuki et al., 2007). It cycles between the PAS and a non-PAS pool and was reported to directly participate in the phagophore formation (Legakis et al., 2007; Mari et al., 2010; Yamamoto et al., 2012). At the non-PAS pool, Atg9 resides in tubular and vesicular structures. These Atg9 reservoirs consist of clustered 20-30 nm vesicles and are often located close to mitochondria. In yeast, they derive from Golgi-related secretory and endosomal pathways, but their origin is still under debate (Mari et al., 2010; Ohashi and Munro, 2010; Yamamoto et al., 2012). At the PAS, Atg9 interacts with the Atg1 kinase complex by direct interaction with the scaffold proteins Atg17 and/or Atg11 (He et al., 2006; Sekito et al., 2009). In addition, Atg1 contains a C-terminal EAT (early autophagosome targeting/tethering) region that has been reported to selectively bind highly curved $20-30 \mathrm{~nm}$ vesicles and to be capable of tethering them (Ragusa et al., 2012). In the current model, the Atg9 containing vesicles are brought together at the PAS mediated by lipid binding of Atg1 and additional protein interaction with Atg17. The vesicles then fuse in a probably SNARE- and the tethering proteins Ypt1 and Trs85-dependent manner to form the phagophore, that finally expands to an autophagosome (Kakuta et al., 2012; Lipatova et al., 2012; Lynch-Day et al., 2010; Ragusa et al., 2012) (Figure 4).

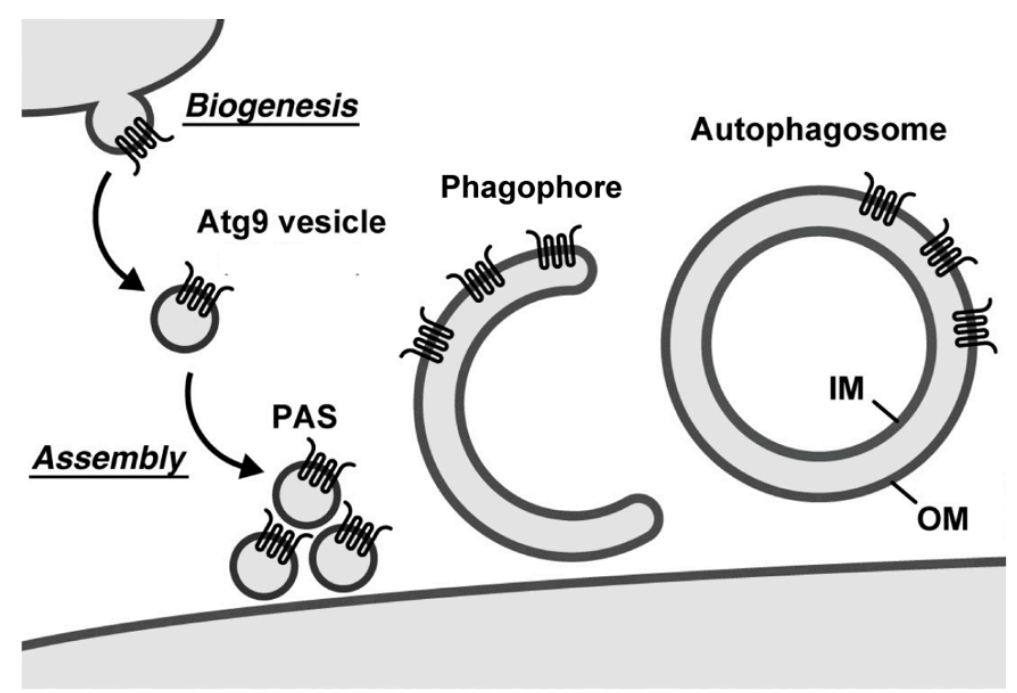

Figure 4: Atg9-containing vesicles contribute in membrane dynamics in autophagy

(IM=inner autophagosomal membrane; OM=outer autophagosomal membrane) ((Yamamoto et al., 2012); modified) 
However, quantitative fluorescence microscopy revealed that only three Atg9 vesicles contribute to the formation of a single autophagosome during starvation (Yamamoto et al., 2012). Based on the reported small diameter of these vesicles, most likely essential amounts of the phagophore lipids derive from other membrane sources (Stanley et al., 2013). Atg9 vesicles are translocated to the PAS immediately after assembly of the scaffold complex, but before recruitment of further Atg protein. Therefore, Atg9 may play a crucial role in a very early step of autophagosome formation such as nucleation of the phagophore or recruitment of other Atg proteins to the PAS (Yamamoto et al., 2012).

The peripherally membrane associated protein Atg23 and the type I membrane protein Atg27 form a trimeric complex with Atg9 that links both proteins (Legakis et al., 2007) (Figure 5). Interestingly, Atg9 belongs to the core autophagy machinery, but Atg27 is only essential for the Cvt pathway (Legakis et al., 2007; Yen et al., 2007).

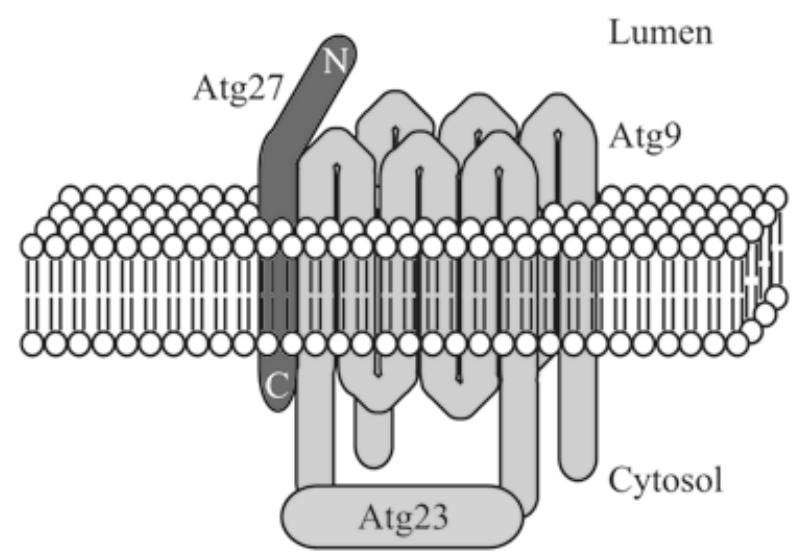

Figure 5: Model of the Atg23-Atg9-Atg27 complex ((Legakis et al., 2007); modified)

As Atg9, Atg23 and Atg27 cycle between the non-PAS pool and the PAS and the cycling of all three proteins depends upon one another. Atg23 and Atg27 are essential for both, transport of Atg9 vesicles from the Golgi to its reservoir (Ragusa et al., 2012; Stanley et al., 2013; Yamamoto et al., 2012) and from its reservoir to the PAS (Legakis et al., 2007). After autophagosome formation, Atg9 clusters on the outer membrane of the autophagosomes and recruits the Atg2Atg18 complex, which mediates together with Atg1 the Atg9 recycling by an unknown mechanism (Ragusa et al., 2012; Stanley et al., 2013; Yamamoto et al., 2012). 


\subsubsection{The Atg14-containing phosphoinositide 3-kinase complex}

The yeast S. cerevisiae has only one phosphoinositide 3-kinase, named Vps34, which specifically phosphorylates phosphoinositides at the D-3 position of the inositol ring to create phosphatidylinositol-3-phosphate (PI3P) (Schu et al., 1993). Vps34 is present in two distinct complexes, which share the subunits Vps34, Vps15 and Atg6. Each complex additionally contains a unique factor, which specifies the localization and cellular function of the respective complex (Kihara et al., 2001) (Figure 6).

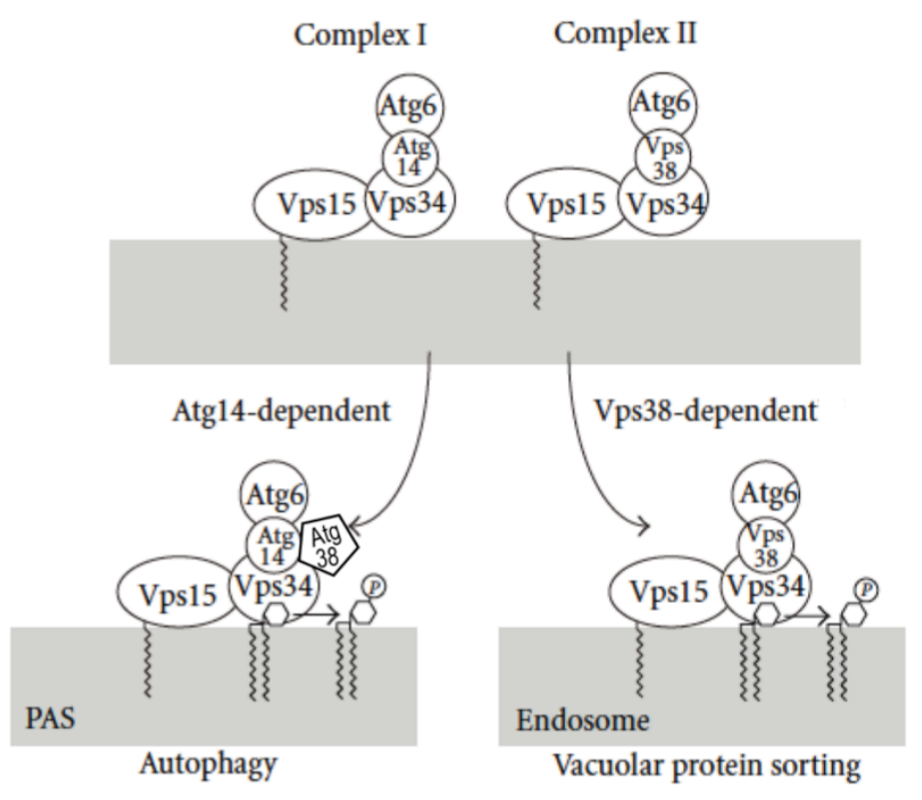

Figure 6: Vps34 is present in two complexes required for different cellular processes ((Obara et al., 2011); modified)

Complex II, containing Vps38, localizes to endosomes and acts in the vacuolar protein sorting pathway (Kihara et al., 2001; Obara et al., 2006). Complex I specifically integrates Atg14 and Atg38 and is essential for autophagy (Araki et al., 2013; Kihara et al., 2001). Atg14 links complex I to the PAS, where it generates the PAS-specific PI3P pool (Jao et al., 2013; Obara et al., 2006). PI3P is essential for autophagy since it recruits autophagy relevant PI3P effectors to the PAS. Deletion of ATG14 and therefore the PI3P pool at the PAS, affects the recruitment of Atg8, the Atg12-Atg5/Atg16 complex and the Atg2-Atg18 complex (see below) to the PAS leading to a complete block in the autophagic process (Suzuki et al., 2007). Consequently, the deletion of VPS38 does not affect autophagy, whereas in the absence of Atg14 the vacuolar protein sorting is unaffected (Kihara et al., 2001). This provides a suitable tool to distinguish 
between the two Vps34 complexes. Furthermore, the influence of the PASspecific PI3P pool on the localization of particular proteins can be specifically investigated.

\subsubsection{The Atg12- and Atg8-conjugating system}

With the Atg12- and Atg8-conjugating systems, autophagy uses two unique ubiquitin-like protein conjugation procedures, which involve approximately half of the core autophagy machinery. The primary sequence of both, Atg12 and Atg8, has low similarity to ubiquitin, but the crystal structure of the mammalian Atg8 homologue (LC3) revealed a C-terminal ubiquitin-like domain for LC3/Atg8. Compared to ubiquitin they additionally contain a N-terminal helical domain (Sugawara et al., 2004). Similarly, based on the plant Atg12 homologue (ATG12b), Atg12 is proposed to contain a ubiquitin-like fold at its C-terminus (Suzuki et al., 2005). Furthermore, Atg12 and Atg8 are conjugated to their specific targets in a manner similar to protein ubiquitination (Figure $7 \mathrm{~A}$ ).

A

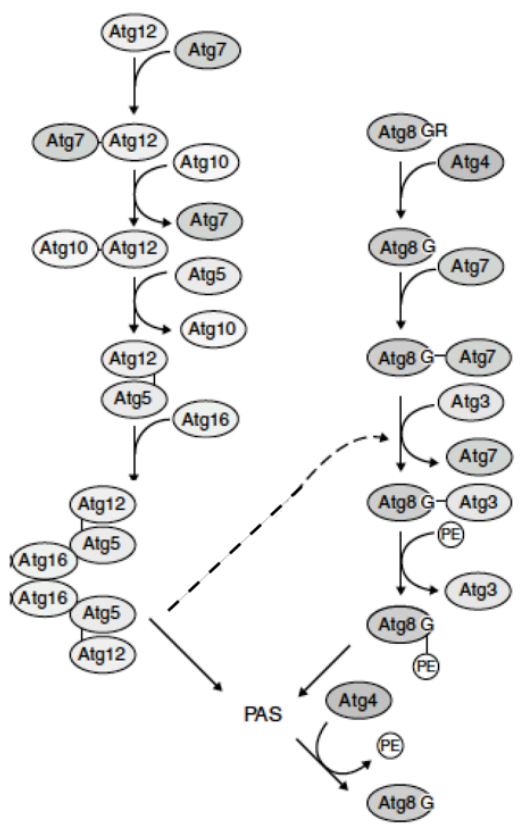

B

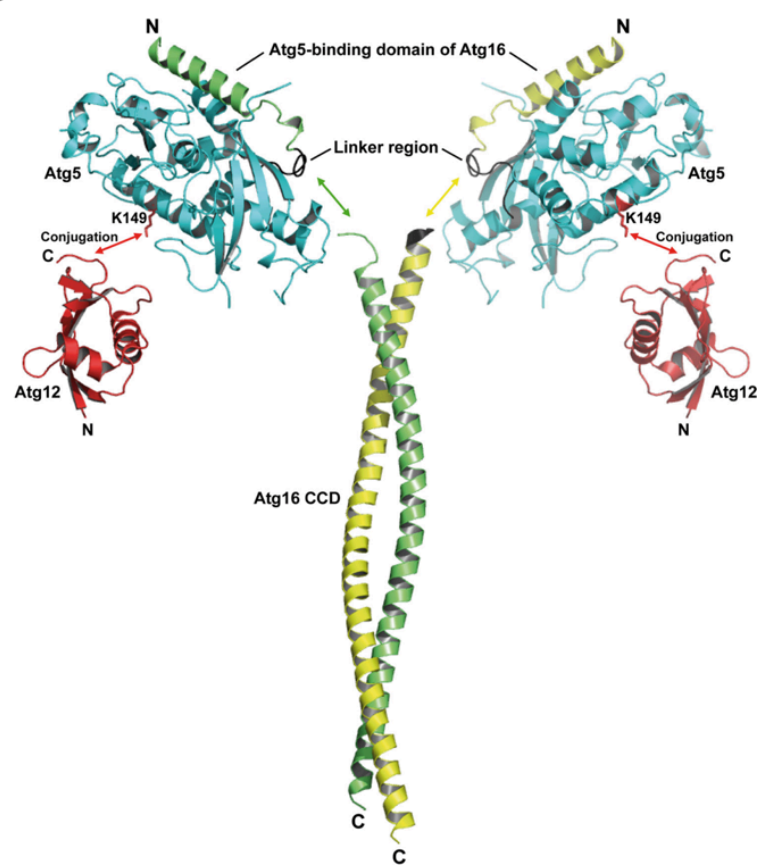

Figure 7: The two ubiquitin-like protein conjugation machineries in autophagy

(A) Scheme of both ubiquitin-like conjugation reactions ((Yorimitsu and Klionsky, 2005); modified) (B) Model of the Atg12-Atg5/Atg16 complex; CCD: coiled-coil domain (Fujioka et al., 2010) 
The C-terminal glycine of Atg12 is activated by the E1 enzyme Atg7, transferred to the E2 enzyme Atg10 and finally covalently attached to an internal lysine of the target protein Atg5 (Kuma et al., 2002; Mizushima et al., 1998; Shintani et al., 1999; Tanida et al., 1999). Remarkably, no E3 enzyme is involved in the conjugation reaction. The substrate specificity is mediated by direct recognition of the target protein Atg5 by the E2 enzyme Atg10 (Yamaguchi et al., 2012). Atg5, preferentially as Atg12-Atg5 conjugate, non-covalently binds to the N-terminus of Atg16 (Atg12-Atg5/Atg16 complex) (Mizushima et al., 1999). Atg12Atg5/Atg16 is most likely a dimeric complex mediated by dimerization of the Cterminal coiled-coil domain of Atg16 (Fujioka et al., 2010; Kuma et al., 2002) (Figure $7 \mathrm{~B}$ ). The dimerization of Atg16 is crucial for the Atg12-Atg5/Atg16 complex activity as an E3-like enzyme in the Atg8 conjugation reaction (Kuma et al., 2002).

Atg8 is synthesized with an additional arginine at its C-terminus. Before conjugation reaction, the cysteine protease Atg 4 cleaves this arginine resulting in an exposed C-terminal glycine (Kirisako et al., 2000). This glycine is also activated by the E1 enzyme Atg7, transferred to the unique E2 enzyme Atg3 and finally covalently attached to the head group of the membrane lipid phosphatidylethanolamine (PE) (Ichimura et al., 2000; Kim et al., 1999; Kirisako et al., 2000; Shintani et al., 1999; Tanida et al., 1999). The conjugation reaction of Atg8 represents a unique mechanism different to ubiquitination as it results in a covalent membrane anchoring of Atg8 via conjugation to a lipid and not a protein.

As mentioned before, the Atg12-Atg5/Atg16 complex promotes this conjugation as an E3-like enzyme. Atg12 directly binds and probably activates Atg3, so that the Atg12-Atg5/Atg16 complex may act as a platform to bring the Atg8-carrying E2 enzyme Atg3 into close proximity to the substrate PE (Noda et al., 2012; Romanov et al., 2012; Sakoh-Nakatogawa et al., 2013). The Atg12-Atg5 conjugate alone was shown to accelerate the Atg8-PE conjugation in vitro, revealing that Atg16 is dispensable for the E3-like function of the Atg12-Atg5 conjugate. However, as a prerequisite for the conjugation of Atg8 to PE, Atg16 directs the Atg12-Atg5/Atg16 complex to autophagy-related membranes in vivo (Hanada et al., 2007). The Atg16-mediated PAS localization of the Atg12-Atg5/Atg16 
complex is dependent on Atg14 respectively the PAS-specific PI3P pool. Because Atg16 has no obvious PI3P binding motif, the mechanism of membrane association remains still elusive (Cebollero et al., 2012; Matsushita et al., 2007; Suzuki et al., 2001).

Atg8-PE is found on all autophagy-related membranes (Kabeya et al., 2000; Kirisako et al., 1999). At the forming autophagosomes, it is located on the inner and outer membrane (Kirisako et al., 2000). The part, present on the inner membrane of the expanding phagophore, is trapped inside the matured autophagosome and transported to the vacuole, where it is finally degraded (Huang et al., 2000; Kabeya et al., 2000; Kirisako et al., 1999). In contrast, the part of Atg8, located on the outer membrane of the forming autophagosome, is cleaved off by the protease Atg4 and subsequently used for another round of lipidation (Kirisako et al., 2000). Beside the recycling aspect, the release of Atg8 from the outer membrane of the expanding phagophore is crucial to facilitate the maturation into a fusion-capable autophagosome (Nair et al., 2012; Yu et al., 2012).

The expression of Atg8 is highly upregulated upon autophagy induction (Huang et al., 2000; Kirisako et al., 1999) and the amount of Atg8 directly correlates with the size of the autophagosomes, suggesting that Atg8 plays a role in autophagosome expansion (Nakatogawa et al., 2007; Xie et al., 2008). In addition, the part of Atg8, which is present on the inner membrane of the forming autophagosome, binds receptor proteins in selective types of autophagy and serves therefore as an adaptor to link the specific cargoes to the forming autophagosomes (Chang and Huang, 2007; Mijaljica et al., 2012; Motley et al., 2012; Shintani et al., 2002). Almost all of the Atg8-interacting proteins bind to a certain region within Atg8 via a particular binding motif, called AIM (Atg8interacting motif) or, with regard to the mammalian Atg8 homologue, LIR (LC3interacting region). The AIM typically consists of a four amino acid motif, beginning with a tryptophan, followed by two, often acidic amino acids and ending with a lysine (WXXL) (Alemu et al., 2012; Mijaljica et al., 2012; Noda et al., 2008). This binding motif is specific for Atg8 homologues, since the side chains of the tryptophan and the lysine within the AIM bind to hydrophobic pockets in 
Atg8, which are conserved among all Atg8 homologues but not among other ubiquitin-like proteins (Noda et al., 2009).

\subsubsection{The Atg2-Atg18 complex}

The Atg18-family proteins belong to the family of $\beta$-propellers that bind polyphosphoinositides (PROPPINs). PROPPINs are conserved from yeast to humans. With Atg18, Atg21 and Hsv2, yeast contains three PROPPINs (Dove et al., 2009; Dove et al., 2004; Krick et al., 2006; Michell et al., 2006; Stromhaug et al., 2004). Mammalia exhibits four PROPPIN orthologs, called WIPIs (WD40 repeat-containing protein that interacts with PtdIns) (Jeffries et al., 2004; Proikas-Cezanne et al., 2004). WIPI1 and WIPI2 share common ancestry with Atg18, whereas WIPI3 and WIPI4 form a clade with Hsv2 (Krick et al., 2012; Polson et al., 2010). Atg21 orthologs, however, have been only found in yeast species so far (Meijer et al., 2007).

Atg18 and its homologues contain seven WD-40 repeats, each consisting of around 40 amino acids and ending with a tryptophan (W) and an aspartate (D) residue. They fold into a seven-bladed $\beta$-propeller. The blades are comprised of four-stranded antiparallel $\beta$-sheets, and are interconnected through six loops (Barth et al., 2001; Dove et al., 2004). Like members of the PROPPIN family from other species, they contain a conserved lipid binding motif, consisting of the four amino acid Phe-Arg-Arg-Gly (FRRG) (Dove et al., 2004; Krick et al., 2006; Nair et al., 2010; Stromhaug et al., 2004). Recent studies revealed, that this motif participate in two different lipid binding sites (Baskaran et al., 2012; Krick et al., 2012; Watanabe et al., 2012), which preferentially bind to PI3P and PI(3,5) $\mathrm{P}_{2}$ and mediate their peripheral membrane association (Dove et al., 2004; Krick et al., 2006) (Figure 8). 
A

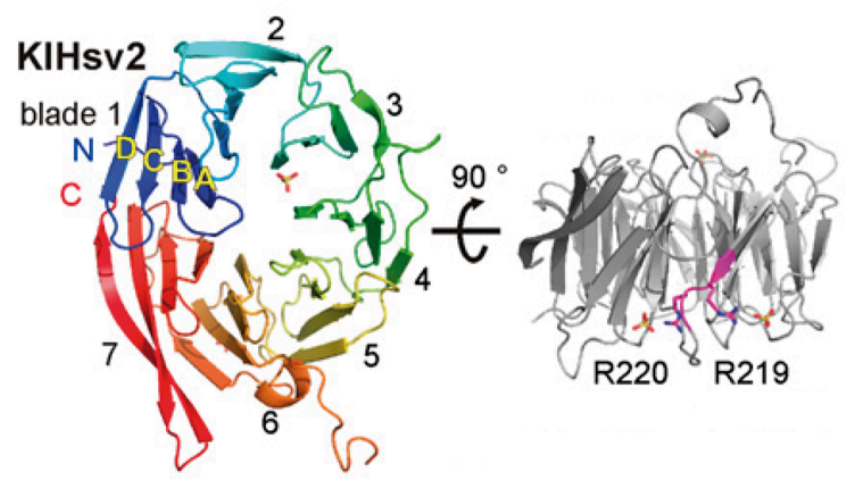

B

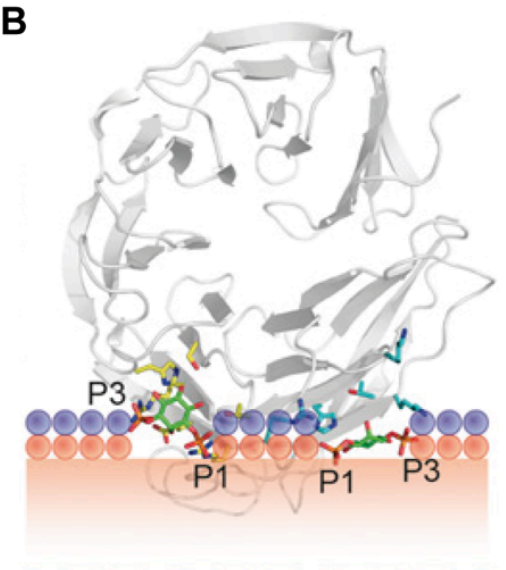

Figure 8: Structure of Kluyveromyces lactis Hsv2

(A) KlHsv2 forms a seven-bladed $\beta$-propeller scaffold. Each blade consists of a four-stranded antiparallel $\beta$-sheet (A-D). The lipid binding motif (FRRG) is located within the fifth loop between blade 5 and 6 and participate in two different lipid-binding pockets. (B) Model of membrane recognition of PROPPINs. The headgroup groups of the two PI3P molecules are indicated in green. ((Krick et al., 2012); modified)

WD-40 domain containing proteins commonly serve as a stable protein-protein interaction platform to coordinate the assembly of protein complexes $\mathrm{Xu}$ and Min, 2011).

Atg18, Atg21 and Hsv2 are highly homologues, but they are required for different types of autophagy. Atg18 as a member of the core autophagy machinery is essential for all types of autophagy, whereas Hsv2 only affects efficient PMN (Barth et al., 2001; Guan et al., 2001; Krick et al., 2008b; Stromhaug et al., 2004). Bulk macroautophagy is severely impaired in the absence of Atg21. In contrast, Atg21 is required for selective types of autophagy as the Cvt pathway or PMN. Consequently, the biogenesis of Cvt vesicles is blocked in the absence of Atg21. In detail, Atg21 is essential for the recruitment of Atg8 and Atg5 to the PAS under nutrient-rich conditions and efficient lipidation of Atg8, suggesting a crucial role for Atg21 in PAS organization during the Cvt pathway. Though, Atg21 has not been detected at the PAS so far (Barth et al., 2002; Krick et al., 2008b; Meiling-Wesse et al., 2004; Stromhaug et al., 2004).

All three homologues localize at endosomal compartments in a PI3P-dependent manner, but their function there remains elusive (Krick et al., 2008a). 
Atg18 also localizes PI(3,5) $\mathrm{P}_{2}$ - and PI3P-dependent at the vacuolar membrane and the PAS, where it exhibits autophagic and non-autophagic functions (Dove et al., 2004; Guan et al., 2001). At the vacuolar membrane it is involved in retrograde vesicular transport from the vacuole to the Golgi and in vacuole homeostasis by regulating the PI3P 5-kinase Fab1 (Dove et al., 2004; Efe et al., 2007; Jin et al., 2008; Michell and Dove, 2009). Atg21 is also found at vertices of vacuolar junctions. However, Atg21 is not required for the retrograde transport from the vacuole as its homolog Atg18 (Dove et al., 2004; Krick et al., 2006; Meiling-Wesse et al., 2004). At the PAS, Atg18 in a complex with Atg2 is required for the retrograde transport of Atg9 from the PAS to its non-PAS pool, but the underlying mechanism remains elusive so far (Legakis et al., 2007; Reggiori et al., 2004). In addition to PI3P, the protein interaction partner Atg2 is required for proper PAS localization of Atg18 (Obara et al., 2008b; Rieter et al., 2013). Atg2 interacts with the transmembrane protein Atg9, thereby mediating the membrane association of the Atg18-Atg2 complex (Obara et al., 2008b; Reggiori et al., 2004). The proper PAS localization of Atg18 requires both, PI3P and the protein interaction partner Atg2. The absence of only one of the components leads to a cytosolic localization and as a consequence to a block in autophagosome biogenesis at an early step (Rieter et al., 2013). Atg18 and Atg2 have been reported to constitutively form a complex in the cytosol, which is subsequently recruited to the PAS dependent on the PI3P binding ability of Atg18 (Obara et al., 2008b). In contrast, recent studies revealed, that Atg2 is recruited to the PAS independently from Atg18 and that the presence of both, Atg2 and PI3P, cooperatively mediates Atg18 PAS recruitment (Rieter et al., 2013). Furthermore, the Atg2-binding site within Atg18 has been located in the loop connecting blade 2 and 3 of the $\beta$-propeller and therefore on the opposite side to the PIP binding region located in loop 5 (Rieter et al., 2013; Watanabe et al., 2012) (Figure 8 A+B). Thus, a simultaneous binding of PIP and Atg2 should be possible.

Not only the localization of Atg18 at the PAS is mediated by PI3P and a protein interaction partner. The localization at the vacuolar membrane is determined by binding to $\mathrm{PI}(3,5) \mathrm{P}_{2}$ and interaction with the PI3P 5-kinase Fab1 complex (Dove et al., 2004; Efe et al., 2007; Krick et al., 2008a). At endosomes the interaction 
partner of Atg18 and the other homologues Atg21 and Hsv2 are still missing. PI3P and $\mathrm{PI}(3,5) \mathrm{P}_{2}$ are enriched at the PAS, endosomes and the vacuolar membrane (Gillooly et al., 2000; Obara and Ohsumi, 2008). Therefore, the correct temporal and spatial PROPPIN localization in the living cell seems to be mainly determined by the particular protein interaction partner (Krick et al., 2012; Rieter et al., 2013). 


\section{Materials and Methods}

\subsection{Material}

\subsubsection{Saccharomyces cerevisiae strains}

Table 1: Yeast strains used in this study

\begin{tabular}{|c|c|c|}
\hline S. cerevisiae strain & Genotype & Reference \\
\hline WCG4 & $\begin{array}{l}\text { WCG4a MAT } \alpha \text { his 2-11,15 leu 2-3,112 } \\
\text { ura } 3\end{array}$ & (Thumm et al., 1994) \\
\hline $\operatorname{atg} 1 \Delta$ & $\begin{array}{l}\text { WCG4a MAT } \alpha \text { his 2-11,15 leu 2-3,112 } \\
\text { ura } 3 \text { atg1 }:: K A N\end{array}$ & (Straub et al., 1997) \\
\hline $\operatorname{atg} 2 \Delta \operatorname{atg} 21 \Delta$ & $\begin{array}{l}\text { WCG4a MAT } \alpha \text { his 2-11,15 leu 2-3,112 } \\
\text { ura } 3 \text { atg2 }:: H I S M X 6 \text { atg21 }:: K A N\end{array}$ & $\begin{array}{l}\text { AG Thumm (University } \\
\text { Göttingen) }\end{array}$ \\
\hline $\operatorname{atg} 3 \Delta$ & $\begin{array}{l}\text { WCG4a MAT } \alpha \text { his 2-11,15 leu 2-3,112 } \\
\text { ura } 3 \text { ade } 2 \Delta 1 \text { atg } 3 \Delta 1:: A D E 2\end{array}$ & (Schlumpberger et al., 1997) \\
\hline $\operatorname{atg} 4 \Delta$ & $\begin{array}{l}\text { WCG4a MAT } \alpha \text { his 2-11,15 leu 2-3,112 } \\
\text { ura } 3 \text { atg } 4 \Delta:: H I S M X 6\end{array}$ & $\begin{array}{l}\text { T. Lang (AG Thumm, } \\
\text { University Göttingen) }\end{array}$ \\
\hline $\operatorname{atg} 5 \Delta$ & $\begin{array}{l}\text { WCG4a MAT } \alpha \text { his 2-11,15 leu 2-3,112 } \\
\text { ura } 3 \text { atg5 }:: K A N\end{array}$ & $\begin{array}{l}\text { AG Thumm (University } \\
\text { Göttingen) }\end{array}$ \\
\hline $\operatorname{atg} 8 \Delta$ & $\begin{array}{l}\text { WCG4a MAT } \alpha \text { his 2-11,15 leu 2-3,112 } \\
\text { ura } 3 \text { atg } 8 \Delta:: K A N\end{array}$ & $\begin{array}{l}\text { AG Thumm (University } \\
\text { Göttingen) }\end{array}$ \\
\hline $\operatorname{atg} 8 \Delta \operatorname{atg} 16 \Delta$ & $\begin{array}{l}\text { WCG4a MAT } \alpha \text { his 2-11,15 leu 2-3,112 } \\
\text { ura } 3 \text { atg8D::KAN atg164::NatNT2 }\end{array}$ & $\begin{array}{l}\text { AG Thumm (University } \\
\text { Göttingen) }\end{array}$ \\
\hline $\operatorname{atg} 8 \Delta \operatorname{atg} 21 \Delta$ & $\begin{array}{l}\text { WCG4a MAT } \alpha \text { his 2-11,15 leu 2-3,112 } \\
\text { ura } 3 \text { atg8D::KAN atg214::NatNT2 }\end{array}$ & $\begin{array}{l}\text { AG Thumm (University } \\
\text { Göttingen) }\end{array}$ \\
\hline $\operatorname{atg} 9 \Delta$ & $\begin{array}{l}\text { WCG4a MAT } \alpha \text { his 2-11,15 leu 2-3,112 } \\
\text { ura } 3 \text { atg9 }:: K A N\end{array}$ & $\begin{array}{l}\text { AG Thumm (University } \\
\text { Göttingen) }\end{array}$ \\
\hline $\operatorname{atg} 11 \Delta$ & $\begin{array}{l}\text { WCG4a MAT } \alpha \text { his 2-11,15 leu 2-3,112 } \\
\text { ura } 3 \text { atg114::HISMX6 }\end{array}$ & $\begin{array}{l}\text { AG Thumm (University } \\
\text { Göttingen) }\end{array}$ \\
\hline $\operatorname{atg} 14 \Delta$ & $\begin{array}{l}\text { WCG4a MAT } \alpha \text { his 2-11,15 leu 2-3,112 } \\
\text { ura } 3 \text { atg144::NatNT2 }\end{array}$ & this study \\
\hline $\operatorname{atg} 164$ & $\begin{array}{l}\text { WCG4a MAT } \alpha \text { his 2-11,15 leu 2-3,112 } \\
\text { ura } 3 \text { atg164::NatNT2 }\end{array}$ & $\begin{array}{l}\text { AG Thumm (University } \\
\text { Göttingen) }\end{array}$ \\
\hline Atg16-HA & $\begin{array}{l}\text { WCG4a MAT } \alpha \text { his 2-11,15 leu 2-3,112 } \\
\text { ura } 3 \text { ATG16-6xHA::NatNT2 }\end{array}$ & $\begin{array}{l}\text { R. Krick (AG Thumm, } \\
\text { University Göttingen) }\end{array}$ \\
\hline Atg161-57-HA & $\begin{array}{l}\text { WCG4a MAT } \alpha \text { his 2-11,15 leu 2-3,112 } \\
\text { ura } 3 \text { ATG161-57-6xHA::NatNT2 }\end{array}$ & this study \\
\hline
\end{tabular}


Materials and Methods

\begin{tabular}{|c|c|c|}
\hline Atg161-119-HA & $\begin{array}{l}\text { WCG4a MAT } \alpha \text { his 2-11,15 leu 2-3,112 } \\
\text { ura } 3 \text { ATG161-119-6xHA::NatNT2 }\end{array}$ & this study \\
\hline Atg16-HA atg8 & $\begin{array}{l}\text { WCG4a MAT } \alpha \text { his 2-11,15 leu 2-3,112 } \\
\text { ura } 3 \quad \text { ATG16-6xHA::NatNT2 } \\
\text { atg8D::KAN }\end{array}$ & this study \\
\hline $\operatorname{atg} 19 \Delta$ & $\begin{array}{l}\text { WCG4a MAT } \alpha \text { his 2-11,15 leu 2-3,112 } \\
\text { ura } 3 \text { atg194::KAN }\end{array}$ & $\begin{array}{l}\text { AG Thumm (University } \\
\text { Göttingen) }\end{array}$ \\
\hline $\operatorname{atg} 21 \Delta$ & $\begin{array}{l}\text { WCG4a MAT } \alpha \text { his 2-11,15 leu 2-3,112 } \\
\text { ura } 3 \text { atg214::KAN }\end{array}$ & (Barth et al., 2002) \\
\hline $\operatorname{atg} 21 \Delta$ рер $4 \Delta$ & 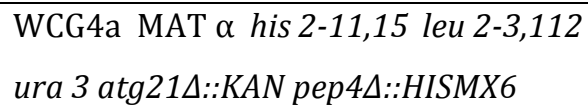 & $\begin{array}{l}\text { AG Thumm (University } \\
\text { Göttingen) }\end{array}$ \\
\hline $\operatorname{atg} 23 \Delta$ & $\begin{array}{l}\text { WCG4a MAT } \alpha \text { his 2-11,15 leu 2-3,112 } \\
\text { ura } 3 \text { atg23D::KAN }\end{array}$ & $\begin{array}{l}\text { AG Thumm (University } \\
\text { Göttingen) }\end{array}$ \\
\hline $\operatorname{atg} 27 \Delta$ & $\begin{array}{l}\text { WCG4a MAT } \alpha \text { his 2-11,15 leu 2-3,112 } \\
\text { ura } 3 \text { atg27L::HISMX6 }\end{array}$ & $\begin{array}{l}\text { AG Thumm } \text { (University } \\
\text { Göttingen) }\end{array}$ \\
\hline Atg27-HA & $\begin{array}{l}\text { WCG4a MAT } \alpha \text { his 2-11,15 leu 2-3,112 } \\
\text { ura } 3 \text { ATG27-6xHA::NatNT2 }\end{array}$ & $\begin{array}{l}\text { R. Krick (AG Thumm, } \\
\text { University Göttingen) }\end{array}$ \\
\hline Sey 6210 & $\begin{array}{l}\text { Sey } 6210 \text { MAT } \alpha \text { ura 3-52 leu 2-3,112 } \\
\text { his 3- } \Delta 200 \text { lys 2-801 trp 1- } \Delta 901 \text { suc 2- } \\
\triangle 9 \text { mel GAL }\end{array}$ & $\begin{array}{l}\text { G. Fischer von Mollard } \\
\text { (University Bielefeld) }\end{array}$ \\
\hline $\operatorname{atg} 4 \Delta$ & $\begin{array}{l}\text { Sey } 6210 \text { MAT } \alpha \text { ura 3-52 leu 2-3,112 } \\
\text { his 3- } \Delta 200 \text { lys 2-801 trp 1- }-901 \text { suc 2- } \\
\triangle 9 \text { mel GAL atg } 4 \Delta:: N a t N T 2\end{array}$ & (Krick et al., 2010) \\
\hline $\operatorname{atg} 5 \Delta$ & 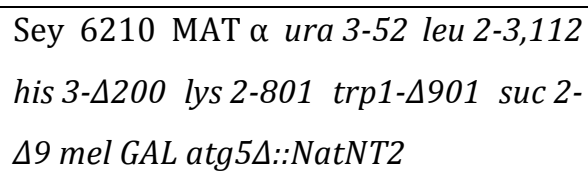 & this study \\
\hline $\operatorname{atg} 16 \Delta$ & $\begin{array}{l}\text { Sey } 6210 \text { MAT } \alpha \text { ura 3-52 leu 2-3,112 } \\
\text { his 3- } \Delta 200 \text { lys 2-801 trp 1- } 4901 \text { suc2- } \\
\Delta 9 \text { mel GAL atg164::NatNT2 }\end{array}$ & this study \\
\hline Atg27-GFP & $\begin{array}{l}\text { ATTC201388 Mat a ura } 3 \text { leu } 2 \text { his } 3 \\
\text { ATG27-GFP::HIS3MX6 }\end{array}$ & Invitrogen \\
\hline Atg27-GFP atg21ه & $\begin{array}{l}\text { ATTC201388 Mat a ura } 3 \text { leu } 2 \text { his } 3 \\
\text { ATG27-GFP::HIS3MX6 ATG21::NatNT2 }\end{array}$ & this study \\
\hline
\end{tabular}




\subsubsection{Escherichia coli strains}

Table 2: E. coli strains used in this study

\begin{tabular}{lll}
\hline E. coli strain & Genotyp & Reference \\
\hline DH5 $\alpha$ & $\mathrm{F}^{\prime}(\Phi$ 80 (UlacZ) M15) $\Delta$ (lacZYA-argF) U169 & (Hanahan, 1983) \\
& recA1 endA1 hsdR17 $r_{K}-m_{K}+$ supE44 thi-1 gyrA & \\
& $r e l A$ & \\
\hline BL21 (DE3) pLysS & $\mathrm{F}^{\prime} d c m$ ompT hsdSB (rB-, mB-) gal $\lambda(D E 3) ;$ & Stratagene \\
& $p L y s S(C a m R)$ & \\
\hline \hline
\end{tabular}

\subsubsection{Plasmids}

Table 3: Plasmids used in this study

\begin{tabular}{|c|c|c|}
\hline Name & Genotype & Reference \\
\hline ApeI-RFP & pRS313 CEN6 HIS3 ApeI-RFP & $\begin{array}{l}\text { AG Thumm } \\
\text { (University Göttingen) }\end{array}$ \\
\hline ApeI-RFP & pRS315 CEN6 LEU2 ApeI-RFP & $\begin{array}{l}\text { AG Thumm } \\
\text { (University Göttingen) }\end{array}$ \\
\hline ApeI-YFP & pRS313 CEN6 HIS3 ApeI-YFP & $\begin{array}{l}\text { AG Thumm } \\
\text { (University Göttingen) }\end{array}$ \\
\hline Atg4 C-YC & p426 2 $\mu$ URA3 ADH Atg4 C-YC & this study \\
\hline Atg5-Cub & pRS313 CEN6 HIS3 MET25 Atg5-Cub-RURA3 & this study \\
\hline Atg5-YFP & pRS316 CEN6 URA3 Atg5-YFP & $\begin{array}{l}\text { Y. Ohsumi } \\
\text { (Tokyo Institute of } \\
\text { Technology) }\end{array}$ \\
\hline Atg16-Cub & pRS313 CEN6 HIS3 MET25 Atg16-Cub-RURA3 & this study \\
\hline Atg16-GFP & pRS313 CEN6 HIS3 Atg16-GFP & $\begin{array}{l}\text { AG Thumm } \\
\text { (University Göttingen) }\end{array}$ \\
\hline Atg16-GFPD101A & pRS313 CEN6 HIS3 Atg16-GFPD101A & this study \\
\hline Atg16-GFPE102A & pRS313 CEN6 HIS3 Atg16-GFPE102A & this study \\
\hline Atg16-GFPD101A_E102A & pRS313 CEN6 HIS3 Atg16-GFPD101A_E102A & this study \\
\hline Atg16-GFPK94A & pRS313 CEN6 HIS3 Atg16-GFPK94A & this study \\
\hline Atg16-HA & pRS313 CEN6 HIS3 CUP1 Atg16-HA & this study \\
\hline Atg16-HA $1-57$ & pRS313 CEN6 HIS3 CUP1 Atg16-HA & this study \\
\hline Atg16-HA $1-119$ & pRS313 CEN6 HIS3 CUP1 Atg16-HA 1-119 & this study \\
\hline Atg16-HA $58-150$ & pRS313 CEN6 HIS3 CUP1 Atg16-HA ${ }^{58-150}$ & this study \\
\hline Atg16-HA ${ }^{\mathrm{D} 101 \mathrm{~A}}$ & pRS313 CEN6 HIS3 CUP1 Atg16-HA ${ }^{\text {D101A }}$ & this study \\
\hline Atg16-HA ${ }^{\mathrm{E} 102 \mathrm{~A}}$ & pRS313 CEN6 HIS3 CUP1 Atg16-HA E102A & this study \\
\hline Atg16-HA D101A_E102A & pRS313 CEN6 HIS3 CUP1 Atg16-HA D101A_E102A & this study \\
\hline Atg16-HA ${ }^{\mathrm{K} 94 \mathrm{~A}}$ & pRS313 CEN6 HIS3 CUP1 Atg16-HAK94A & this study \\
\hline
\end{tabular}


Materials and Methods

\begin{tabular}{|c|c|c|}
\hline Atg16-HA & pRS313 CEN6 HIS3 CUP1 Atg16-HAE97A & this study \\
\hline Atg21-Cub & pRS313 CEN6 HIS3 MET25 Atg21-Cub-RURA3 & $\begin{array}{l}\text { R. Krick (AG Thumm, } \\
\text { University Göttingen) }\end{array}$ \\
\hline Atg21 C-YC & p426 $2 \mu$ URA3 ADH Atg21 C-YC & this study \\
\hline Atg21 C-YC & p416 CEN URA3 MET25 Atg21 C-YC & this study \\
\hline Atg21-TAP & pRS316 CEN6 URA3 Atg21-TAP & $\begin{array}{l}\text { AG Thumm } \\
\text { (University Göttingen) }\end{array}$ \\
\hline Atg21-YFP & pRS316 CEN6 URA3 Atg21-YFP & $\begin{array}{l}\text { (Meiling-Wesse et al., } \\
\text { 2004) }\end{array}$ \\
\hline Atg23-Cub & pRS313 CEN6 HIS3 MET25 Atg23-Cub-RURA3 & this study \\
\hline Atg27-Cub & pRS313 CEN6 HIS3 MET25 Atg27-Cub-RURA3 & this study \\
\hline Atg27-Cub $1-198$ & $\begin{array}{lllll}\text { pRS313 } & \text { CEN6 } & \text { HIS3 } & \text { MET25 Atg27-Cub1-198 } \\
\text { RURA3 } & & & & \\
\end{array}$ & $\begin{array}{l}\text { R. Krick (AG Thumm, } \\
\text { University Göttingen) }\end{array}$ \\
\hline Atg27-Cub1-221 & $\begin{array}{llllll}\text { pRS313 } & \text { CEN6 } & \text { HIS3 } & \text { MET25 Atg27 Cub1-221 } \\
\text { RURA3 } & & & & & \\
\end{array}$ & $\begin{array}{l}\text { R. Krick (AG Thumm, } \\
\text { University Göttingen) }\end{array}$ \\
\hline $\mathrm{C}-\mathrm{YC}$ & $\mathrm{p} 4262 \mu$ URA3 ADH C-YC & (Skarp et al., 2008) \\
\hline $\mathrm{C}-\mathrm{YC}$ & p416 CEN6 URA3 C-YC & this study \\
\hline GFP-Atg8 & pRS313 Cen6 HIS3 GFP-Atg8 & $\begin{array}{l}\text { AG Thumm } \\
\text { (University Göttingen) }\end{array}$ \\
\hline GFP & pUG36 CEN6 HIS3 GFP & $\begin{array}{l}\text { AG Thumm } \\
\text { (University Göttingen) }\end{array}$ \\
\hline GFP-Atg21 & pUG36 CEN6 HIS3 GFP-Atg21 & (Krick et al., 2008a) \\
\hline GST & pGEX-4T-3 GST & Amersham \\
\hline GST-Atg16 & pGEX-4T-3 GST-Atg16 & this study \\
\hline GST-Atg161-57 & pGEX-4T-3 GST-Atg161-57 & this study \\
\hline GST-Atg161-119 & pGEX-4T-3 GST-Atg161-119 & this study \\
\hline GST-Atg16 $6^{58-150}$ & pGEX-4T-3 GST-Atg16 ${ }^{58-150}$ & this study \\
\hline mCherry & pUG36 CEN6 URA3 mCherry & $\begin{array}{l}\text { R. Krick (AG Thumm, } \\
\text { University Göttingen) }\end{array}$ \\
\hline mCherry-Atg19 & pUG34 CEN6 HIS3 mCherry-Atg19 & $\begin{array}{l}\text { P. Rube (AG Thumm, } \\
\text { University Göttingen) }\end{array}$ \\
\hline mCherry-Atg21 & pUG36 CEN6 URA3 mCherry-Atg21 & $\begin{array}{l}\text { R. Krick (AG Thumm, } \\
\text { University Göttingen) }\end{array}$ \\
\hline Nui-Atg1 & pRS314 CEN6 TRP1 CUP1 Nui-Atg1 & $\begin{array}{l}\text { F. Reggiori } \\
\text { (University Medical } \\
\text { Center Utrecht) }\end{array}$ \\
\hline Nui-Atg5 & pRS314 CEN6 TRP1 CUP1 Nui-Atg5 & $\begin{array}{l}\text { R. Krick (AG Thumm, } \\
\text { University Göttingen) }\end{array}$ \\
\hline
\end{tabular}


Materials and Methods

\begin{tabular}{|c|c|c|}
\hline Nui-Atg7 & pRS314 CEN6 TRP1 CUP1 Nui-Atg7 & this study \\
\hline Nui-Atg8 & pRS314 CEN6 TRP1 CUP1 Nui-Atg8 & $\begin{array}{l}\text { R. Krick (AG Thumm, } \\
\text { University Göttingen) }\end{array}$ \\
\hline Nui-Atg9 & pRS314 CEN6 TRP1 CUP1 Nui-Atg9 & $\begin{array}{l}\text { F. Reggiori } \\
\text { (University Medical } \\
\text { Center Utrecht) }\end{array}$ \\
\hline Nui-Atg12 & pRS314 CEN6 TRP1 CUP1 Nui-Atg12 & this study \\
\hline Nui-Atg16 & pRS314 CEN6 TRP1 CUP1 Nui-Atg16 & this study \\
\hline Nui-Atg18 & pRS314 CEN6 TRP1 CUP1 Nui-Atg18 & $\begin{array}{l}\text { F. Reggiori } \\
\text { (University Medical } \\
\text { Center Utrecht) }\end{array}$ \\
\hline Nui-Atg19 & pRS314 CEN6 TRP1 CUP1 Nui-Atg19 & this study \\
\hline Nui-Atg21 & pRS314 CEN6 TRP1 CUP1 Nui-Atg21 & $\begin{array}{l}\text { F. Reggiori } \\
\text { (University Medical } \\
\text { Center Utrecht) }\end{array}$ \\
\hline Nui-Atg23 & pRS314 CEN6 TRP1 CUP1 Nui-Atg23 & this study \\
\hline Nui-Atg27 & pRS314 CEN6 TRP1 CUP1 Nui-Atg27 & this study \\
\hline Nui-Hsv2 & pRS314 CEN6 TRP1 CUP1 Nui-Hsv2 & $\begin{array}{l}\text { R. Krick (AG Thumm, } \\
\text { University Göttingen) }\end{array}$ \\
\hline Nui-Ubc6 & pRS314 CEN6 TRP1 CUP1 Nui-Ubc6 & $\begin{array}{l}\text { R. Krick (AG Thumm, } \\
\text { University Göttingen) }\end{array}$ \\
\hline Nui-Trs85 & pRS314 CEN6 TRP1 CUP1 Nui-Trs85 & $\begin{array}{l}\text { F. Reggiori } \\
\text { (University Medical } \\
\text { Center Utrecht) }\end{array}$ \\
\hline Nui-Vam1 & pRS314 CEN6 TRP1 CUP1 Nui-Vam1 & $\begin{array}{l}\text { AG Thumm } \\
\text { (University Göttingen) }\end{array}$ \\
\hline $\mathrm{N}-\mathrm{YC}$ & p426 $2 \mu$ URA3 ADH N-YC & (Skarp et al., 2008) \\
\hline $\mathrm{N}-\mathrm{YN}$ & $\mathrm{p} 4252 \mu$ LEU2 ADH N-YN & (Skarp et al., 2008) \\
\hline $\mathrm{N}-\mathrm{YN}$ & p415 CEN6 LEU2 MET25 N-YN & this study \\
\hline N-YN Atg8 & 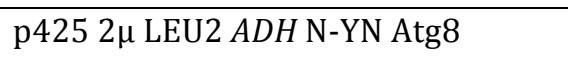 & this study \\
\hline N-YN Atg8 & p415 CEN6 LEU2 MET25 N-YN Atg8 & this study \\
\hline N-YC Atg 4 & p426 $2 \mu$ URA3 ADH N-YC Atg4 & this study \\
\hline N-YC Atg21 & p426 $2 \mu$ URA3 ADH N-YC Atg21 & this study \\
\hline pFA6-natNT2 & pFA6-natNT2 & $\begin{array}{l}\text { Euroscarf; (Janke et al., } \\
2004 \text { ) }\end{array}$ \\
\hline pRS313-CUP1 & pRS313 CEN6 HIS3 CUP1 & $\begin{array}{l}\text { R. Krick (AG Thumm, } \\
\text { University Göttingen) }\end{array}$ \\
\hline pRS314 & pRS314 CEN6 TRP1 & $\begin{array}{l}\text { AG Thumm } \\
\text { (University Göttingen) }\end{array}$ \\
\hline
\end{tabular}


Materials and Methods

\begin{tabular}{l|l|l} 
p415-MET25 & $\begin{array}{l}\text { p415 CEN LEU2 MET25 } \\
672\end{array}$ & (Mumberg et al., 1995) \\
\hline p416-MET25 & $\begin{array}{l}\text { p416 CEN URA3 MET25 } \\
673\end{array}$ & (Mumberg et al., 1995) \\
\hline Ste14-Cub & pRS313 CEN6 HIS3 MET25 Ste14-Cub-RURA3 & $\begin{array}{l}\text { F. Reggiori } \\
\text { (University } \\
\text { Center Utrecht) }\end{array}$ \\
\hline SUM0 & K27 6xHIS SUMO & $\begin{array}{l}\text { AG Rehling } \\
\text { (University Göttingen) }\end{array}$ \\
\hline SUM0-Atg21 & K27 6xHIS SUM0-Atg21 & $\begin{array}{l}\text { R. Krick (AG Thumm, } \\
\text { University Göttingen) }\end{array}$ \\
\hline \hline
\end{tabular}

\subsubsection{Oligonucleotides}

Table 4: Oligonucleotides used in this study

\begin{tabular}{|c|c|}
\hline Name & Sequence ( $5^{`}$ to $3^{\prime}$ ) \\
\hline \multicolumn{2}{|l|}{ ATG5::NatNT2 } \\
\hline Atg5::NAT_fwd & $\begin{array}{l}\text { GTTCTTTTGGTTCTAGAAGAACGGAGATAGGAAACCTATGATGTAAGTAT } \\
\text { GCGTACGCTGCAGGTCGAC }\end{array}$ \\
\hline Atg5::NAT_rev & $\begin{array}{l}\text { CTGCGATATTTGAATGACACTTTTAAATGCGTATATAACAGCTCTTAATC } \\
\text { GATGAATTCGAGCTCG}\end{array}$ \\
\hline \multicolumn{2}{|l|}{ ATG14::NatNT2 } \\
\hline atg14_ko_fw & $\begin{array}{l}\text { CGAGTAGAGAAAAAGGGAAGTAAAAGTTAAAAACTAGAATCCTAGTATG } \\
\text { ACATG CGTACGCT GCAGGTCGAC }\end{array}$ \\
\hline atg14_ko_rev & $\begin{array}{l}\text { GACTGACTACATGCAACTTTATACACACGGCAGGAAAAAAAGTGCGCACT } \\
\text { CTA ATCGATGAATTCGAGCTCG }\end{array}$ \\
\hline \multicolumn{2}{|l|}{ ATG21::NatNT2 } \\
\hline ATG21 KO-S1: & $\begin{array}{l}\text { CAAAAGACAATTCCACTCCTTTGGATTTGAAATAGACAGATAGAAAAGGA } \\
\text { TATGCGTACGCTGCAGGTCGAC }\end{array}$ \\
\hline ATG21 KO-S2: & $\begin{array}{l}\text { CGTGAATACGTACAATATCTATTAAGATTATGAAAACTGCACATATGCAT } \\
\text { TAATCGATGAATTCGAGCTCG }\end{array}$ \\
\hline \multicolumn{2}{|l|}{$\begin{array}{l}\text { chromosomal Atg161- } \\
\text { 119-HA }\end{array}$} \\
\hline Atg161-119-HA-S3_fwd & $\begin{array}{l}\text { GAACCATTGAAAATAATGTTTTACAACAAAAACTCTCGGATCGTACGCTG } \\
\text { CAGGTCGAC }\end{array}$ \\
\hline Atg161-119-HA-S2_rev & $\begin{array}{l}\text { TTAGCCATCTCGCAACTAGCTGGGAATGTTCTTTTTTCAGATCGATGAAT } \\
\text { TCGAGCTCG }\end{array}$ \\
\hline $\begin{array}{l}\text { chromosomal Atg161- } \\
\text { 57-HA }\end{array}$ & \\
\hline
\end{tabular}




\begin{tabular}{|c|c|}
\hline Atg161-57-HA-S3_fwd & $\begin{array}{l}\text { GTTGTTTCAAGATAATAGTGGCGCCATTGGTGGCAACATTCGTACGCTGC } \\
\text { AGGTCGAC }\end{array}$ \\
\hline Atg161-57-HA-S2_rev & $\begin{array}{l}\text { GAATTGCCAGTGTATTCAATAAAGCATCGTCATGGCTGACATCGATGAA } \\
\text { TTCGAGCTCG }\end{array}$ \\
\hline \multicolumn{2}{|l|}{$\begin{array}{l}\text { Split-ubiquitin } \\
\text { plasmids }\end{array}$} \\
\hline Nui-Atg23_BamHI_fwd & AGTAGCAATggatcctgggtctgggGAACTGAATCAGGTTTTAG \\
\hline Nui_Atg23_KpnI_rev & ATTCCTACTTGGTACCGAAAGCCTAGATCATGATC \\
\hline Atg23-Cub_StuI_fwd & AGTAGCAATaggcctATGGAACTGAATCAGGTTTTAG \\
\hline Atg23_Cub_SalI_rev & ATTCCTACTTgtcgacCCTTCAACTTTTTTTGATATGGCATC \\
\hline Nui-Atg12_StuI_fwd & AGTAGGAATggatcaggccttctgggAGTAGGATCCTAGAGAGCG \\
\hline Nui-Atg12_XhoI_rev & ATTCCTACTTCTCGAGgtgagtttataacgctcaagtac \\
\hline Nui-Atg27_BamHI_fwd & AGTAGCAATggatccctgggtctgggGTATCGAAGACTTGGATATGTGG \\
\hline Nui-Atg27_XhoI_rev & ATTCCTACTTCTCGAGgtataatgtagatataaaagcttag \\
\hline Nui-Atg16_BamHI_fwd & AGTAGCAATggatccctgggtctgggGGCAATTTCATTATAACAGAAAGG \\
\hline Nui-Atg16_XhoI_rev & ATTCCTACTTCTCGAGacctctttattgcaaacactttg \\
\hline Nui-Atg19_BamHI_fwd & AGTAGCAATggatcctgggtctgggAACAACTCAAAGACTAACCAACAG \\
\hline Nui-Atg19_XhoI_rev & ATTCCTACTTCTCGAGggaagtaaagagtttcgaaaaggg \\
\hline Nui-Atg7_BamHI_fwd & AGTAGCAATggatccctgggtctgggTCGTCAGAAAGGGTCTTAAG \\
\hline Nui-Atg7_KpnI_rev & ATTCCTACTTGGTACCGTATGCAAAATAGTATAGCAAG \\
\hline Atg5Cub_StuI_fwd & AGTAGCAATaggcctATGAATGACATTAAACAATTACTTTG \\
\hline Atg5Cub_SalI_rev & ATTCCTACTTgtcgacCCGAGCTCAGAGGAAGCTTTATC \\
\hline Atg16Cub_ClaI_fwd & AGTAGCAATatcgatATGGGCAATTTCATTATAACAG \\
\hline Atg16Cub_SalI_rev & ATTCCTACTTgtcgacCCTTTCGTTCCATCTATTTCGCTG \\
\hline Atg27Cub_ClaI_fwd & AGTAGCAATatcgatATGGTATCGAAGACTTGGATATG \\
\hline Atg27Cub_SalI_rev & ATTCCTACTTgtcgacCCAACGGCGCTATAACCGCCTC \\
\hline \multicolumn{2}{|l|}{$\begin{array}{l}\text { GST-Atg16 plasmid } \\
\text { variants }\end{array}$} \\
\hline GST-Atg16_BamHI_fwd & AGTAGCAATggatccATGGGCAATTTCATTATAACAG \\
\hline GST-Atg16_XhoI_rev & ATTCCTACTTCTCGAGTCATTTCGTTCCATCTATTTCG \\
\hline GST-Atg161-57_XhoI_rev & ATTCCTACTTCTCGAGTCAAATGTTGCCACCAATGGCG \\
\hline $\begin{array}{l}\text { GST-Atg16 }{ }^{1-} \\
{ }^{119} \text { XhoI_rev }\end{array}$ & ATTCCTACTTCTCGAGTCAATCCGAGAGTTTTTGTTGTAAAAC \\
\hline $\begin{array}{l}\text { GST-Atg16 } 58- \\
\text { 150_BamHI_fwd }\end{array}$ & AGTAGCAATggatccGTCAGCCATGACGATGC \\
\hline \multicolumn{2}{|l|}{$\begin{array}{l}\text { Atg16-HA plasmid } \\
\text { variants }\end{array}$} \\
\hline Atg16Cub_ClaI_fwd & AGTAGCAATatcgatATGGGCAATTTCATTATAACAG \\
\hline
\end{tabular}




\begin{tabular}{|c|c|}
\hline Atg16HA_XhoI_rev & AGTAGCAATctcgagGATCTCTTGAATGATCGTTCC \\
\hline $\begin{array}{l}\text { Atg16HA_58- } \\
\text { 150_ClaI_fwd }\end{array}$ & AGTAGCAATatcgatATGGTCAGCCATGACGATGC \\
\hline \multicolumn{2}{|l|}{ Atg16 point mutants } \\
\hline Atg16_K94A_fwd & GCGTTGAAAAATgcGAATACGGAAAGGTTGAATGACG \\
\hline Atg16_K94A_rev & CGTCATTCAACCTTTCCGTATTCGCATTTTTCAACGC \\
\hline Atg16_E97A_fwd & GAATACGGcAAGGTTGAATGACGAATTGATTAGTGGAACCATTG \\
\hline Atg16_E97A_rev & CAATGGTTCCACTAATCAATTCGTCATTCAACCTTGCCGTATTC \\
\hline Atg16_D101A_fwd & GAATACGGAAAGGTTGAATGcCGAATTGATTAGTGGAACC \\
\hline Atg16_D101A_rev & GGTTCCACTAATCAATTCGgCATTCAACCTTTCCGTATTC \\
\hline Atg16_E102A_fwd & GAATACGGAAAGGTTGAATGACGcATTGATTAGTGGAACC \\
\hline Atg16_E102A_rev & GGTTCCACTAATCAATgCGTCATTCAACCTTTCCGTATTC \\
\hline Atg16_DE_fwd & GAATACGGAAAGGTTGAATGcCGcATTGATTAGTGGAACCATTG \\
\hline Atg16_DE_rev & CAATGGTTCCACTAATCAATgCGgCATTCAACCTTTCCGTATTC \\
\hline \multicolumn{2}{|l|}{ BiFC plasmids } \\
\hline $\begin{array}{l}\text { Atg21_C- } \\
\text { YC_BamHI_fwd }\end{array}$ & atcatcggatccATGAAAGTATTACAATTCAATCAAG \\
\hline Atg21_C-YC_SalI_rev & gatgatgtcgacTGTAAATTTATTATTTTTAGTCAGCAC \\
\hline $\begin{array}{l}\text { Atg21_N- } \\
\text { YC_BamHI_fwd }\end{array}$ & atcatcggatccCAAAGTATTACAATTCAATCAAGATG \\
\hline Atg21_N-YC_SalI_rev & gatgatgtcgacTTATGTAAATTTATTATTTTTAGTCAGC \\
\hline Atg8_N-YN_BamHI_fwd & atcatcggatccAAGTCTACATTTAAGTCTGAATATC \\
\hline Atg8_N-YN_SalI_rev & atcatcggatccCTACCTGCCAAATGTATTTTCTC \\
\hline Atg4_C-YC_EcoRI_fwd & atcatcgaattcATGCAGAGGTGGCTACAACTG \\
\hline Atg4_C-YC_SalI_rev & gatgatgtcgacgcatttttcatcaataggactgtg \\
\hline Atg4 N-YC_EcoRI_fwd & atcatcgaattcGCAGAGGTGGCTACAACTGTGG \\
\hline Atg4 N-YC_SalI_rev & gatgatgtcgacCTAGCATTTTTCATCAATAGGACTG \\
\hline \multicolumn{2}{|l|}{ Control Primer } \\
\hline Atg5_K1_fwd & GAAGTAGCATGCTCAGAAGTG \\
\hline NatNT2_r & cgattcgtcgtccgattcgtc \\
\hline Atg5_K3_rev & CAACGTAGGATTGACTCCAGTC \\
\hline atg14_K1_fw & GGGAAAGGACCAAATACAAAAGTG \\
\hline atg14_K3_rev & TGAGTTGGTTCTTACCCGAATGC \\
\hline Atg21_pro_f & GAGCGTGAGCTGCAGAAAG \\
\hline atg21-seq1r & caacaatttcatgtgggaaaac \\
\hline \multicolumn{2}{|l|}{ Sequencing primer } \\
\hline pRS313-CUP1_fwd & GCAATATGGATTGTCAGAATC \\
\hline pRS313-CUP1_rev & CACACAGGAAACAGCTATGACC \\
\hline
\end{tabular}




\begin{tabular}{|c|c|}
\hline pGEX-4T3_fwd & gatcatgtaacccatcc \\
\hline pGEX-4T3_rev & GCCACCTGACGTCTAAG \\
\hline Atg16 seq 2 rev & CTTCGTATTTCTTGCTCC \\
\hline Nui-Seq3_f & CTTGTCTTGTATCAATTG \\
\hline Atg23_Seq1_fwd & CTTCTGCGGATCAAATGATAC \\
\hline Atg23_seq1_rev & GTATCATTTGATCCGCAGAAG \\
\hline Atg23_Seq2_fwd & GTAGGAGATTTGGAAAGC \\
\hline ATG16_seq_6_rev & GGATAACAATTTCACACAGG \\
\hline Cub-RURA3-seq f & CACCTTGTCCAATTGAAC \\
\hline Cub_Seq_rev & CTAACTCCAGTAATTCCTTG \\
\hline Atg12 Seq $1 \mathrm{fwd}$ & CAAGAATTAAGATCATCTCC \\
\hline Atg12 Seq2 fwd & CGTTTGCGCCAAGTCCGCAG \\
\hline Atg12 Seq2 rev & CTGCGGACTTGGCGCAAACG \\
\hline Atg27 Seq1 fwd & CAGGTGGGAAAATTTAGCTC \\
\hline Atg27 Seq2 fwd & GGACTTCGTGGTTCACTTGG \\
\hline Atg27 Seq2 rev & CCAAGTGAACCACGAAGTCC \\
\hline ATG16 seq 2 for & GGAGCAAGAAATACGAAG \\
\hline Atg16 seq 3 fwd & GCCATGAACAGCGAAATAG \\
\hline Atg16 seq3 rev & CTATTTCGCTGTTCATGGC \\
\hline Atg19 Seq1 fwd & GCTTGGATAACTTCATGAAAC \\
\hline Atg19 Seq1 rev & GTTTCATGAAGTTATCCAAGC \\
\hline Atg19 Seq2 fwd & CCAACCACGCAAATTATTGAC \\
\hline Atg19 Seq2 rev & GTCAATAATTTGCGTGGTTGG \\
\hline Atg7_Seq1_fwd & GGCTGTTCTCAAAGTGTC \\
\hline Atg7_Seq1_rev & GACACTTTGAGAACAGCC \\
\hline Atg7_Seq2_fwd & GTAAACGAGGAGGCTCAG \\
\hline Atg7_Seq2_rev & CTGAGCCTCCTCGTTTAC \\
\hline Atg7_Seq3_fwd & GACTTCCTTACTACAGACC \\
\hline Atg5_Seq1_fwd & CСССТCATTTGGAACAAG \\
\hline Atg5_Seq1_rev & CTTGTTCCAAATGAGGGG \\
\hline Atg5_Seq2_fwd & GTTCATCAAGACCGCGAC \\
\hline Atg5_Seq2_rev & GTCGCGGTCTTGATGAAC \\
\hline pADH 1 for & CGGTATACGGCCTTCCTTCC \\
\hline C-YC Seq rev & CTCGTCCATGCCGAGAGTG \\
\hline N-YC Seq fwd & GTGCAGCTCGCCGACCAC \\
\hline Atg 4 C-YC Seq1 f & CAGAGGTGGCTACAACTGTG \\
\hline Atg4 C-YC Seq1 r & CTATAGGAACAAATCTTGTCCG \\
\hline Atg 4 C-YC Seq2 f & CCGATCAGTACAATAGAGGAC \\
\hline
\end{tabular}




\begin{tabular}{l|l}
\hline Atg4 C-YC Seq2 r & GATGCCACATTCGGGGAAGC \\
\hline Atg4 C-YC Seq3 $\mathrm{f}$ & GTTCTTGTTGGGCGTGAAGC \\
\hline Atg4 C-YC Seq3 r & GTTGCCAGTCTTTTTCGCCC \\
\hline Atg4 C-YC Seq4 $\mathrm{f}$ & GGATGACGTGGAAAGTGTAAG \\
\hline Atg4 C-YC Seq4 $\mathrm{r}$ & CATCAATAGGACTGTGAATACC \\
\hline \hline
\end{tabular}

\subsubsection{Media}

All media listed in this chapter were prepared with deionized water $\left(\mathrm{ddH}_{2} \mathrm{O}\right)$. The respective $\mathrm{pH}$ was adjusted using $\mathrm{NaOH}$ or $\mathrm{HCl}$. The media were autoclaved at $121^{\circ} \mathrm{C}$ for $20 \mathrm{~min}$ for sterilization. The listed percent values indicate weight per volume $(\mathrm{w} / \mathrm{v})$. For the preparation of solid media to generate plates, $2 \%$ of preheated, sterile agar was added.

\subsubsection{YPD-medium, pH 5.5}

YPD is a rich medium for yeast cells consisting of:

$1 \%$ Bacto $^{\circledR}$ Yeast Extract

2\% Bacto ${ }^{\circledR}$ Pepton

$2 \%$ D-glucose

\subsubsection{CM-medium, pH 5.6}

CM-medium is a synthetic medium for yeast cells and was used as selective medium.

$0.67 \%$ Yeast Nitrogen Base w/o amino acids

$2 \%$ D-glucose

$0.0117 \%$ L-alanine

0.0117\% L-methionine

$0.0117 \%$ L-arginine

0.0117\% L-phenylalanine

0.0117\% L-asparagine

0.0117\% L-proline

$0.0117 \%$ L-aspartic acid

$0.0117 \%$ L-serine

$0.0117 \%$ L-cysteine

0.0117\% L-threonine

$0.0117 \%$ L-glutamine

$0.0117 \%$ L-tyrosine

$0.0117 \%$ L-glutamic acid

0.0117\% L-valine

0.0117\% L-glycine

$0.0117 \%$ myo-inositol

0.0117\% L-isoleucine

$0.0117 \%$ p-aminobenzoic acid 
For overexpression experiments using MET25 promoter L-methionine was excluded from the drop out mix.

Following supplements were added depending on selection of genetic markers:

$0.3 \mathrm{mM}$ L-histidine

1.7 mM L-leucine

1 mM L-lysine
0.4 mM L-tryptophan

$0.3 \mathrm{mM}$ adenine

$0.2 \mathrm{mM}$ uracil

\subsubsection{SD(-N)-medium}

Nitrogen free SD(-N)-medium was used as starvation medium for yeast cells.

0.67\% Yeast Nitrogen Base w/o amino acids and w/o ammonium sulfate

$2 \% \quad$ D-glucose

\subsubsection{LB-medium, $\mathrm{pH} 7.5$}

LB-medium was used as standard growth medium for $E$. coli cultures.

1\% Bacto ${ }^{\circledR}$ Trypton

$0.5 \%$ Bacto ${ }^{\circledR}$ Yeast extract

$0.5 \%$ sodium chloride

For plasmid selection $75 \mu \mathrm{g} / \mathrm{ml}$ ampicillin, $50 \mu \mathrm{g} / \mathrm{ml}$ kanamycin, and/or $25 \mu \mathrm{g} / \mathrm{ml}$ chloramphenicol was added.

\subsubsection{SOC-medium, $p H 7.5$}

SOC-medium was used as regeneration medium for electroporated E. coli cells.

2\% Bacto ${ }^{\circledR}$ Trypton

$0.5 \%$ Bacto ${ }^{\circledR}$ Yeast extract

$0.4 \%$ D-glucose

10 mMsodium chloride

$10 \mathrm{mM}$ magnesium sulfate

10 mMmagnesium chloride

$2.5 \mathrm{mM}$ potassium chloride

\subsubsection{Antibodies}

Table 5: Antibodies used in this study

\begin{tabular}{l|l|l} 
Antibody & $\begin{array}{l}\text { Dilution } \\
\text { (in TBST containing 1\% } \\
\text { skim milk powder (w/v)) }\end{array}$ & Source \\
\hline \hline anti-mouse-HRPO-conjugate & $1: 10.000$ & Dianova, Hamburg \\
\hline anti-rabbit-HRPO-conjugate & $1: 5000$ & Medac, Hamburg \\
\hline anti-rat-HRPO-conjugate & $1: 10.000$ & Jackson ImmunoResearch, UK \\
\hline \hline
\end{tabular}


Materials and Methods

\begin{tabular}{l|l|l}
\hline rabbit-anti-ApeIp & $1: 3000$ & Eurogentech, Belgium \\
\hline rabbit-anti-Aut7 & $1: 10.000$ in TBST & M. Thumm \\
\hline rabbit-anti-CPY & $1: 10.000$ & Molecular Probes, Leiden, NL \\
\hline mouse-anti-GFP* & $1: 10.000$ & Roche, Mannheim \\
\hline mouse-anti-GFP** & $1: 10.000$ & Abcam, Cambridge, UK \\
\hline mouse-anti-PGK & $1: 10.000$ & Molecular Probes, Leiden, NL \\
\hline rat-anti-Red & $1: 1000$ & Chromotek, München \\
\hline mouse-anti-HA & $1: 10.000$ & $\begin{array}{l}\text { Santa Cruz Bio-technology, } \\
\text { Heidelberg }\end{array}$ \\
\hline \hline
\end{tabular}

*In general, the GFP-antibody from Roche was used if not stated otherwise.

${ }^{* *}$ The GFP-antibody from Abcam was used to detect both fragments of eYFP on immunoblots prepared for BiFC (see chapter 4.3.5.1).

\subsubsection{Commercial available Kits}

Kit systems listed in Table 6 were used according to the manufacturer`s recommendations.

Table 6: Commercial available Kits used in this study

\begin{tabular}{l|l} 
Name of the Kit & Source \\
\hline ECL Western Blotting Detection & Amersham Biosciences, GB \\
Reagents & \\
\hline QIAquick Gel Extraction Kit & Qiagen, Hilden \\
\hline QIAquick PCR Purification Kit $\quad$ Qiagen, Hilden \\
\hline QuikChange II Agirected & \\
Mutagenesis Kit & Promega, Mannheim \\
\hline Wizard Plus SV Miniprep Kit &
\end{tabular}

\subsubsection{Chemicals and consumables}

Standard chemicals were used in analytical grade quality and obtained from AppliChem (Darmstadt), Sigma (Deisenhofen), Roth (Karlsruhe) or Merck (Darmstadt). Restriction enzymes were obtained from NEB (Frankfurt). Deoxyoligonucleotides were ordered from Eurofins MWG Operon (Ebersberg) and used as primers for PCR or site-directed mutagenesis. Special chemicals and enzymes are listed in Table 7. 


\section{Materials and Methods}

Table 7: Special chemicals

\begin{tabular}{|c|c|}
\hline Name & Source \\
\hline Bacto $^{\circledR}$-Agar & Becton Dickinson, Heidelberg \\
\hline Bacto ${ }^{\circledR}$ Peptone & Becton Dickinson, Heidelberg \\
\hline Bacto ${ }^{\circledR}$ Tryptone & Becton Dickinson, Heidelberg \\
\hline Bacto ${ }^{\circledR}$ Yeast Extract & Becton Dickonson, Heidelberg \\
\hline Benzonase & Sigma, Deisenhofen \\
\hline clon NAT (nourseotricine) & Werner BioAgents, Jena \\
\hline Complete $^{\mathrm{TM}}$ protease inhibitor (EDTA-free) & Roche, Mannheim \\
\hline Deoxyadenosin-triphosphate (dATP) & NEB, Frankfurt \\
\hline Deoxycytidin-triphosphate (dCTP) & NEB, Frankfurt \\
\hline Deoxyguanosin-triphosphate (dGTP) & NEB, Frankfurt \\
\hline Deoxythymidin-triphosphate (dTTP) & NEB, Frankfurt \\
\hline DNA-marker (1kb DNA-ladder) & NEB, Frankfurt \\
\hline ECL & USB, Santa Clara, CA \\
\hline Glass beads & Schütt, Göttingen \\
\hline Herring-sperm-DNA & Promega, Madison, USA \\
\hline Immersion oil & Applied Precision, USA \\
\hline Ligation buffer & NEB, Frankfurt \\
\hline DNA polymerase (FideliTaq) & USB, Santa Clara, USA \\
\hline DNA polymerase (Klenow) & NEB, Frankfurt \\
\hline DNA polymerase (KOD) & Novagen, Darmstadt \\
\hline DNA polymerase (Taq) & NEB, Frankfurt \\
\hline DNA polymerase (Vent) & NEB, Frankfurt \\
\hline Precision Plus Protein All Blue Standards & Biorad, Munich \\
\hline Protease inhibitor cocktail (bacteria) & Sigma, Deisenhofen \\
\hline RNAse A & Applichem, Darmstadt \\
\hline Sepharose / Slurry & GE Healthcare, München \\
\hline Skim milk powder & Granovita, Lüneburg \\
\hline T4-Ligase & NEB, Frankfurt \\
\hline Difco Yeast nitrogen base w/o amino acids & Becton Dickinson, Heidelberg \\
\hline Difco Yeast nitrogen base e/o amino acids and ammonium & Becton Dickinson, Heidelberg \\
\hline Zymolyase T100 & Seikagaku, Japan \\
\hline
\end{tabular}




\subsubsection{Equipment}

Table 8: Equipment used in this study

Name of product

\begin{tabular}{|c|c|}
\hline $\begin{array}{l}\text { Agarose gel equipment } \\
\text { Bio RAD Mini-SUB Cell GT }\end{array}$ & Bio-Rad Laboratories GmbH, München \\
\hline ÄKTApurifier 10 UPC & GE Healthcare, München \\
\hline Autoclave & Adolf Wolf, SANOclav, Bad Überkingen-Hausen \\
\hline Autoclave DX200 & Systec, Wettenberg \\
\hline Bench & $\begin{array}{l}\text { BDK Luft- und Reinraumtechnik GmbH, } \\
\text { Sonnenbühl }\end{array}$ \\
\hline Blot Shaker GFL 3019 & GFL, Burgwedel \\
\hline Centrifuge 5804 & Eppendorf, Hamburg \\
\hline Centrifuge 5404R & Eppendorf, Hamburg \\
\hline Centrifuge 5415D & Eppendorf, Hamburg \\
\hline Centrifuge $5415 \mathrm{R}$ & Eppendorf, Hamburg \\
\hline Chemical balance & Sartorius, Göttingen \\
\hline Cuvettes no. 67.742 & Sarstedt, Nümbrecht \\
\hline Cuvettes for electroporation; $2 \mathrm{~mm}$ & peqlab, Erlangen \\
\hline Electroporator 2510 & Eppendorf; Hamburg \\
\hline Freezer $\left(-20^{\circ} \mathrm{C}\right)$ & Liebherr, Bulle, CH \\
\hline Freezer $\left(-80^{\circ} \mathrm{C}\right)$ & Heareus, Hanau \\
\hline Glassbeads & Schütt, Göttingen \\
\hline Hood & $\begin{array}{l}\text { BDK Luft- und Reinraumtechnik, Sonnenbrühl- } \\
\text { Genkingen }\end{array}$ \\
\hline Incubator $\left(37^{\circ} \mathrm{C}\right)$ & Heraeus, Hanau \\
\hline Incubator 4200 & Innova, USA \\
\hline Incubator Thermomixer comfort & Eppendorf, Hamburg \\
\hline Labshaker for diverse culture sizes & A. Kühner, Birsfelden, Schweiz \\
\hline LAS 3000 Intelligent Dark Box & Fuji/Raytest, Benelux \\
\hline Magnetic stirrer MR 3001 & Heidolph, Kelheim \\
\hline Microscope DeltaVision, Olympus IX71 & Applied Precision, USA \\
\hline Microscope slides (76x26mm) & Menzel-Gläser, Braunschweig \\
\hline Microscope cover slips & Menzel-Gläser, Braunschweig \\
\hline Microwave R-939 & Sharp, Hamburg \\
\hline Multivortex IKA vibray VXR basic & IKA, Staufen \\
\hline Over head shaker Roto-Shake Genie & Scientific Industries Inc, USA \\
\hline PCR Mastercycler gradient & Eppendorf, Hamburg \\
\hline pH meter pH537 & WTW, Weilheim \\
\hline
\end{tabular}

\section{Source}

Source




\begin{tabular}{|c|c|}
\hline Photometer & Eppendorf, Hamburg \\
\hline Pipettes & Eppendorf, Hamburg \\
\hline PowerPac Basic Power Supply & Bio-Rad Laboratories GmbH, München \\
\hline PowerPac HC Power Supply & Bio-Rad Laboratories GmbH, München \\
\hline PVDF membrane Hybond-P & Amersham; GE healthcare, Freiburg \\
\hline Refrigerator $\left(4^{\circ} \mathrm{C}\right)$ & Bosch, Stuttgart / Liebherr, Bulle, CH \\
\hline Rotor JA 10 & Beckmann, Krefeld \\
\hline Rotor JA 20 & Beckmann, Krefeld \\
\hline Rotor TLA-100.3 & Beckmann, Krefeld \\
\hline Rotor TLS-55 & Beckmann, Krefeld \\
\hline OmniTrays Nunc & SIGMA-ALDRICH, St. Louis, USA \\
\hline Superose ${ }^{\mathrm{TM}} 610 / 300 \mathrm{GL}$ column & Amersham Pharmacia Biotech, Schweden \\
\hline $\begin{array}{l}\text { SDS-PAGE equipment BioRAD Mini } \\
\text { Protean cell }\end{array}$ & Bio-Rad Laboratories GmbH, München \\
\hline Pipette tips, petri dishes, ... & Sarstedt, Nümbrecht / Eppendorf, Hamburg \\
\hline Sterile filter & Whatman, GE healthcare, München \\
\hline Thermomixer Comfort & Eppendorf, Hamburg \\
\hline Transilluminator TI 1 & Whatman Biometra, Göttingen \\
\hline Ultracentrifuge & Beckman, Krefeld \\
\hline vacuum pump & Vacuubrand, Wertheim \\
\hline Water bath SWB25 & Thermo Electron, Karlsruhe \\
\hline Western Blot equipment Trans Blot Cell & Bio-Rad Laboratories GmbH, München \\
\hline
\end{tabular}

\subsection{Cultivation of $S$. cerevisiae}

\subsubsection{Growth of liquid yeast cultures}

For liquid yeast precultures, the yeast strain was inoculated with a sterile toothpick from an agar plate and incubated at $30^{\circ} \mathrm{C}$ with $220 \mathrm{rpm}$ over night. The preculture was used to inoculate the main liquid yeast culture with a defined dilution depending on the requested $\mathrm{OD}_{600}$ and growth ability of the respective strain. Cultures were shaken at $220 \mathrm{rpm}$ and $30^{\circ} \mathrm{C}$ over night $(12-14 \mathrm{~h})$ if not pointed otherwise.

\subsubsection{Short-term storage}

For short-term storage of yeast stocks, yeast strains were stored on agar plates up to 4 - 6 weeks at $4^{\circ} \mathrm{C}$. 


\subsubsection{Long-term storage}

For long-term storage of yeast strains, $0.65 \mathrm{ml}$ of a yeast culture was supplemented with $0.65 \mathrm{ml}$ of sterile $30 \%$ glycerine and stored at $-80^{\circ} \mathrm{C}$.

\subsubsection{Cell density determination}

The cell density was determined by $\mathrm{OD}_{600}$ measurement using a photometer in a dilution of 1:10. The empty medium was used as reference. $1 \mathrm{OD}_{600}$ of yeast cells corresponds to $3 \times 10^{7}$ cells per ml.

\subsubsection{Split-ubiquitin assay}

$1 \mathrm{OD}_{600}$ of a yeast preculture was diluted 1:10, 1:100, 1:1000 and 1:10 000 with sterilized $\mathrm{ddH}_{2} 0.4 \mu \mathrm{l}$ was spotted on three different solid plates (Laser et al., 2000):

1) growth control plate consisting of selective medium (CM-His-Trp)

2) negative interaction control plate consisting of selective medium (CM-His-Trp) lacking uracil but containing $250 \mu \mathrm{M}$ methionine and $100 \mathrm{MM} \mathrm{CuSO}_{4}$

3) positive interaction control plate consisting of selective medium (CM-His-Trp) containing $250 \mu \mathrm{M}$ methionine, $100 \mu \mathrm{M} \mathrm{CuSO}_{4}$ and $1 \mathrm{mg} / \mathrm{ml} \mathrm{FOA}$

Plates were incubated for two to three days at $30^{\circ} \mathrm{C}$ and imaged using LAS-3000 (Fujifilm) for growth pattern analysis.

\subsection{Cultivation of $E$. coli cultures}

\subsubsection{Growth of liquid $E$. coli cultures}

Liquid E. coli cultures were inoculated with cells from an agar plate, a short-term or long-term culture using a sterile toothpick. The cells were shaken at $37^{\circ} \mathrm{C}$ and $220 \mathrm{rpm}$ over night $(12-14 \mathrm{~h})$ or for a depicted time period in LB medium containing the appropriate antibiotic/s for selection.

\subsubsection{E. coli short-term storing}

For short-time storage, over night liquid cultures were stored at $4^{\circ} \mathrm{C}$ for up to 4 weeks. 


\subsubsection{E. coli long-term storing}

For long-term storage, $0.65 \mathrm{ml}$ of an over night liquid culture was supplemented with $0.65 \mathrm{ml}$ of sterile $60 \%$ glycerine and stored at $-80^{\circ} \mathrm{C}$.

\subsubsection{Preparation of electrocompetent $E$. coli cells}

Competent E. coli cells were prepared from a $1 \mathrm{l}$ culture of $\mathrm{OD}_{600} \sim 0.6$. Cells were cooled down on ice for $10 \mathrm{~min}$ and afterwards harvested by centrifugation ( $8 \mathrm{~min}, 6500 \mathrm{rpm}, 4^{\circ} \mathrm{C}$ ). They were washed two times with ice-cold $\mathrm{ddH}_{2} \mathrm{O}$ and once with ice-cold 10\% (v/v) glycerine. Finally, cells were resuspended in $2 \mathrm{ml}$ ice-cold $10 \%(\mathrm{v} / \mathrm{v})$ glycerine, split into $40 \mu \mathrm{l}$ aliquots and stored at $-80^{\circ} \mathrm{C}$.

\subsection{Microscopy}

\subsubsection{Fluorescence microscopy}

An essential part of this work represented the in vivo visualization and evaluation of proteins labeled with a fluorescent tag by direct fluorescence microscopy. Yeast cells expressing the desired fluorescent-tagged proteins were grown over night at $30^{\circ} \mathrm{C}$ in $\mathrm{CM}$ selection media to the preferred growth phase. To cells expressing pMET25-mCherry-Atg21, $0.3 \mathrm{mM}$ methionine was added to induce endogenous protein expression level. 4 - $5 \mu$ l of the culture was dropped on a slide and covered with a cover slip. Pictures were taken using a DeltaVision Spectris fluorescence microscopy (Olympus IX71, Applied Precision) equipped with a CoolSNAP HQ camera and either FITC and TRITC filter or specific GFP, YFP and mCherry filter sets (see Table 9). Images were generating using a $100 \mathrm{x}$ objective by collecting a stack of at least 18 pictures with focal planes $0.20 \mu \mathrm{m}$ apart. Pictures were deconvoluted using SoftWoRx software (Applied Precision) and processed using Adobe Systems Photoshop and Illustrator. A single focal plane is shown at each time. 
Table 9: Overview of the used filter sets in fluorescence microscopy

\begin{tabular}{lll}
\hline Filter Sets & Excitation wavelength $(\mathbf{n m})$ & Emmision wavelength $(\mathbf{n m})$ \\
\hline FITC & $475 / 28$ & $523 / 36$ \\
TRITC & $542 / 27$ & $594 / 45$ \\
GFP & $475 / 28$ & $525 / 50$ \\
YFP & $513 / 17$ & $559 / 38$ \\
mCherry & $575 / 25$ & $632 / 60$ \\
POL & $-50 / 28$ & $-50 / 0$ \\
\hline
\end{tabular}

\subsubsection{Bimolecular Fluorescence Complementation (BiFC)}

Bimolecular Fluorescence Complementation is described as a suitable method to study protein-protein interaction in their cellular environment using direct fluorescence microscopy (Skarp et al., 2008). For this purpose, two proteins of interest were fused to one fragment of eYFP. Expression vectors allowing highcopy expression of the fusion protein tagged either at the amino- or carboxy terminus with eYFP fragments from a constitutive $A D H$ promotor were kindly provided by Skarp et al. (2008). In addition, expression vectors enable inducible expression of the tagged target proteins from a MET25 promotor were used. Constructs were made as described in chapter 3.5.10.2.

Cells expressing fusion proteins from the $A D H$ promoter were grown to log phase and visualized using a DeltaVision Deconvolution microscope as described in chapter 3.4.1.

Cells expressing fusion proteins from the MET25 promoter were grown to midlog phase in CM selective medium containing an excess of methionine. Cells were subsequently diluted to an $\mathrm{OD}_{600}$ of 0.3 in $\mathrm{CM}$ selective medium containing $0.5 \mathrm{mM}$ methionine to induce fusion protein expression. Cells were grown at $30^{\circ} \mathrm{C}$ for an indicated period of time and visualized using a DeltaVision Deconvolution microscope as described in chapter 3.4.1.

For determination of the expression level of the fusion proteins, cells were grown in selective medium containing an indicated amount of methionine at $30^{\circ} \mathrm{C}$ over night to mid-log phase. Cells were subsequently alkaline lysed and the samples subjected to immunoblotting and analyzed using anti-GFP antibody (Abcam). 


\subsubsection{Determination of colocalization rates}

In this study, direct fluorescence microscopy was predominantly used for localization studies of a particular protein. For this, the localization of this protein was determined by colocalization with other proteins that served as a reference like a PAS marker.

For the PAS localization studies of Atg21-YFP, first the percentage of cells positive for both, an Atg21-YFP and ApeI-RFP signal (PAS rate), was determined by analyzing at least 130 cells from at least three independent experiments. Subsequently, the quantity of ApeI-RFP dots colocalized with Atg21-YFP was determined (Colocalization Rate).

Similarly, the PAS and colocalization rate of mCherry-Atg21 and ApeI-YFP was determined as described above by analyzing at least 190 cells from three independent experiments.

To determine the Atg5/Atg16 rate, at least 322 cells from three independent experiments were observed for a perivacuolar Atg5-YFP respectively Atg16-GFP puncta.

For colocalization studies of mCherry-Atg21 and Atg16-GFP respectively Atg5YFP, the quantity of Atg16-GFP respectively Atg5-YFP dots colocalized with mCherry-Atg21 was determined by analyzing at least 350 cells from at least four independent experiments.

For colocalization studies of mCherry-Atg21 and cAtg27-GFP, first the quantity of mCherry-Atg21 dots colocalized with cAtg27-GFP per cell was determined by analyzing 76 cells from two independent experiments. These cells were aligned into four categories:

1) no mCherry-Atg21 dot colocalized with cAtg27-GFP

2) $1-49 \%$ of the mCherry-Atg21 dots colocalized with cAtg27-GFP

3) $49-99 \%$ of the mCherry-Atg21 dots colocalized with cAtg27-GFP

4) all of the mCherry-Atg21 dots colocalized with cAtg27-GFP

\subsection{Molecular biology methods}

\subsubsection{Isolation of chromosomal DNA}

For the isolation of chromosomal DNA, $1.5 \mathrm{ml}$ of an over night liquid yeast culture was harvested by centrifugation (13200 rpm, $1 \mathrm{~min}$ ), washed once with 
$\mathrm{ddH}_{2} \mathrm{O}$ and resuspended in $200 \mu \mathrm{l}$ breaking buffer $(10 \mathrm{mM}$ Tris/HCl pH 8.0, $100 \mathrm{mM} \mathrm{NaCl}, 1 \mathrm{mM}$ EDTA, 1\% SDS, 2\% Triton X-100). After addition of $200 \mu \mathrm{l}$ phenol/chloroform solution (50\% (v/v) phenol, 50\% (v/v) chloroform) and $200 \mu \mathrm{l}$ glass beads, the cells were lysed by harsh mixing for $20 \mathrm{~min}$ at $4^{\circ} \mathrm{C} .200 \mu \mathrm{l}$ $\mathrm{ddH}_{2} \mathrm{O}$ was added and the sample was centrifuged for $10 \mathrm{~min}$ at $13200 \mathrm{rpm}$. The upper phase was transferred into a new reaction tube, mixed with $1 \mathrm{ml}$ ice-cold ethanol and incubated for $10 \mathrm{~min}$ at $-20^{\circ} \mathrm{C}$ for precipitation of DNA. After centrifugation for $10 \mathrm{~min}$ at $13200 \mathrm{rpm}$ and $4^{\circ} \mathrm{C}$ the supernatant was removed. The pellet was resuspended in $400 \mu \mathrm{lddH} 20$ and $3 \mu \mathrm{l} \mathrm{RNase} \mathrm{A} \mathrm{(10} \mathrm{mg/ml)} \mathrm{and}$ incubated for $5 \mathrm{~min}$ at $37^{\circ} \mathrm{C}$ to digest the RNA. For further precipitation of the remaining DNA, $1 \mathrm{ml}$ of ice-cold ethanol and $10 \mu \mathrm{l} 5 \mathrm{M}$ ammonium acetate were added. After incubation of $15 \mathrm{~min}$ at $-20^{\circ} \mathrm{C}$, the DNA was harvested by centrifugation for $10 \mathrm{~min}$ at $13200 \mathrm{rpm}$ and $4^{\circ} \mathrm{C}$. The supernatant was removed, the DNA pellet was dried at $37^{\circ} \mathrm{C}$ and finally resolved in $35 \mu \mathrm{ldd} \mathrm{H}_{2} \mathrm{O}$.

\subsubsection{High efficiency transformation of DNA in yeast}

A $50 \mathrm{ml}$ liquid yeast culture was inoculated from a log phase yeast culture with a dilution of $1: 10$. The cells were grown for $2-6 \mathrm{~h}$ at $30^{\circ} \mathrm{C}$ to an $\mathrm{OD}_{600}$ of $0.5-0.8$ and subsequently harvested by centrifugation ( $5 \mathrm{~min}, 2000 \mathrm{rpm}, \mathrm{RT}$ ). After two washing steps with $\mathrm{ddH}_{2} \mathrm{O}$ and one with LiOAC-Sorb $(100 \mathrm{mM}$ lithiumacetate, $10 \mathrm{mM}$ Tris/acetate pH 8.0, $1 \mathrm{mM}$ EDTA, $1 \mathrm{M}$ sorbitol) the cells were resuspended in $100-500 \mu \mathrm{l}$ LiOAC-Sorb and incubated for $15 \mathrm{~min}$ at $30^{\circ} \mathrm{C}$ for recovery. $50 \mu \mathrm{l}$ cells were used for one transformation reaction. For this, $300 \mu \mathrm{l}$ PEG in Li-TE buffer (100 mM lithiumacetate, $10 \mathrm{mM}$ Tris/acetate pH 8.0, $1 \mathrm{mM}$ EDTA, 40\% PEG 3350), $5 \mu \mathrm{l}$ herring sperm DNA and $3-10 \mu \mathrm{l}$ of the respective DNA were added. The reaction was first incubated for $30 \mathrm{~min}$ at $30^{\circ} \mathrm{C}$ and then shifted to $42^{\circ} \mathrm{C}$ for $15 \mathrm{~min}$. For recovery, the cells were transferred into $2 \mathrm{ml}$ YPD-medium and shaken for $2 \mathrm{~h}$ at $30^{\circ} \mathrm{C}$. Finally, the cells were harvested by centrifugation ( $5 \mathrm{~min}, 2000 \mathrm{rpm}, \mathrm{RT}$ ) and spread on the respective selection medium.

\subsection{3 “Quick and Dirty" transformation of plasmid-DNA in yeast}

For the fast transformation of plasmid-DNA, yeast cells were taken from an agar plate and resuspended in $300 \mu \mathrm{l}$ PEG in Li-TE buffer $(100 \mathrm{mM}$ lithiumacetate, 
$10 \mathrm{mM}$ Tris/acetate pH 8.0, 1 mM EDTA, 40\% PEG 3350). $5 \mu \mathrm{l}$ of herring sperm DNA and $3-5 \mu \mathrm{l}$ of the plasmid/s were added. After gently mixing, the cells were incubated for $30 \mathrm{~min}$ at $30^{\circ} \mathrm{C}$ and then shifted to $42^{\circ} \mathrm{C}$. Afterwards, cells were

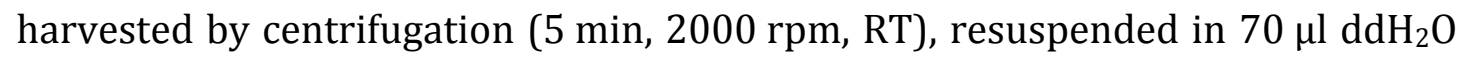
and directly spread on the respective selection medium.

\subsubsection{Transformation of plasmid-DNA into $E$. coli}

In this study, transformation of plasmid-DNA in E. coli was achieved by electroporation. Here, the permeability of the cell membrane is increased by an applied electric impulse resulting in the uptake of external DNA (Sheng et al., 2005). For transformation, electrocompetent E. coli cells (see 3.3.4) were thawed on ice, mixed with 1 - $2 \mu \mathrm{l}$ of the appropriate DNA and transferred to a prechilled electroporation tube. The electroporation occurred at $2500 \mathrm{~V}$. The cells were resuspended in $900 \mu \mathrm{l}$ SOC-medium and shaken for $1 \mathrm{~h}$ at $37^{\circ} \mathrm{C}$ for recovery. After harvesting by centrifugation ( $5 \mathrm{~min}, 3000 \mathrm{rpm}$, RT), the cells were spread on LB-plates containing the appropriate antibiotics for plasmid selection.

\subsubsection{Plasmid-DNA isolation from $E$. coli}

Liquid E. coli cultures were inoculated with cells from either an agar plate using a sterile toothpick, from a short-term culture or from a long-term culture. The cells were shaken at $37^{\circ} \mathrm{C}$ over night $(12-14 \mathrm{~h})$ at $220 \mathrm{rpm}$. Plasmid-DNA was isolated using the Wizard Plus SV Kit from Promega according to the manufacturer's instructions.

\subsubsection{DNA agarose gel electrophoresis}

The DNA agarose gel electrophoresis was used as a standard method to separate DNA fragments according to their size in an electric field. Depending on the size of the DNA fragments, $1-2 \%$ agarose gels supplemented with $1 \mu \mathrm{g} / \mathrm{ml}$ ethidiumbromide in TAE buffer $(40 \mathrm{mM}$ Tris/acetate pH 8.1, $2 \mathrm{mM}$ EDTA, $0.114 \%$ acetic acid) were used. The DNA samples were mixed with DNA sample buffer (1 M Tris/HCl pH 8.0, 50\% (v/v) glycerol, 0.1\% (w/v) bromphenolblue) and separated for $20-30 \mathrm{~min}$ at $120 \mathrm{~V}$. To determine the DNA fragment size, the 
DNA Ladder Mix TriDye (1 kb, NEB) was used. DNA bands were documented by an UV-transilluminator.

\subsubsection{DNA gel extraction (Gel Extraction Kit)}

For purification of DNA fragments from agarose gels, the Qiagen Gel Extraction Kit from Qiagen (Hilden) was used according to the manufacturer's instructions.

\subsubsection{Polymerase chain reaction}

DNA fragments used for molecular cloning or for homologous recombination in yeast were amplified by PCR using the Taq DNA polymerase (NEB) or for longer DNA fragments the KOD Hot Start DNA Polymerase (Novagen) according to the recommendations of the manufacturer. Therefore, standard PCR reactions in a $50 \mu \mathrm{l}$ scale were performed. Plasmid DNA or yeast genomic DNA were used as templates. The respective program was adapted for each PCR reaction depending on oligonucleotides and product size. The amplified DNA was purified using the Qiagen PCR Purification Kit from Qiagen according to the manufacturer's instructions.

\subsubsection{Molecular cloning}

In this study, several plasmids were constructed for the expression of a desired fusion protein (see Table 3). Therefore, the insert DNA sequence was amplified by PCR using chromosomal DNA or another plasmid as template. Here, flanking restriction sites for cloning were introduced by the designed primers (see Table 4). The DNA fragment was analyzed by agarose gel electrophoresis and purified using the Qiagen Gel Extraction Kit (see chapter 3.5.6 + 3.5.7).

The insert DNA fragment and the plasmid backbone were digested with the respective restriction enzymes (NEB) according to the manufacturer's instructions in a scale of $30 \mu \mathrm{l}$. The reaction was incubated for $90 \mathrm{~min}$ at the enzyme optimal temperature. Afterwards the fragments were again purified and used for ligation. For determination of the optimal DNA ratio the following equation was used: 
Equation 1: Equation to calculate optimal plasmid-insert ratio in ligation mix

mass insert $(\mathrm{ng})=\frac{5 \times \text { mass plasmid }(\mathrm{ng}) \times \text { length insert }(\mathrm{bp})}{\text { length plasmid }(\mathrm{bp})} 1$

To the appropriate volumes of plasmid and insert DNA, $1 \mu$ of ligation buffer and 2.5 U T4-DNA-ligase (NEB) were added and the reaction was filled up to $10 \mu \mathrm{l}$ with $\mathrm{ddH}_{2} \mathrm{O}$. The ligation reaction was incubated for $2 \mathrm{~h}$ at room temperature or over night at $16^{\circ} \mathrm{C}$. The ligated constructs were transformed in E. coli Dh5 $\alpha$ by electroporation (see chapter 3.5.4). Potential clones were analyzed by restriction analysis and finally verified by sequencing.

\subsubsection{Plasmid construction}

\subsubsection{Plasmid constructs used in split-ubiquitin assay}

For the construction of all split-ubiquitin plasmids, the plasmids pRS313MET25-STE14-Cub-RURA3 (provided by F. Reggiori (University Medical Centre Utrecht, Utrecht, The Netherlands) and N. Johnsson (Universität Ulm, Ulm, Germany)) and pRS314-CUP1-Nui-UBC6 (provided by N. Johnsson; (Wittke et al., 1999)) were used as initial plasmid backbone. The gene sequence of STE14 was cut out with ClaI and Sall (if not stated otherwise) and replaced with the respective gene sequence. Similarly, the sequence of UBC6 was cut out with BamHI and XhoI, if not stated otherwise.

For MET25-ATG16-Cub-RURA3, ATG16 was amplified with Atg16Cub_ClaI_fwd and Atg16Cub_SalI-rev, cut with ClaI and SalI and ligated in MET25-Cub-RURA3. For MET25-ATG27-Cub-RURA3, ATG27 was amplified with Atg27Cub_ClaI_fwd and Atg27Cub_SalI_rev, cut with ClaI and SalI and ligated in MET25-Cub-RURA3. For MET25-ATG23-Cub-RURA3, ATG23 was amplified with Atg23Cub_StuI_fwd and Atg23Cub_SalI_rev. For MET25-ATG5-Cub-RURA3, ATG5 was amplified with Atg5Cub_StuI_fwd and Atg5Cub_SalI_rev. Both fragment were cut with StuI and SalI and ligated in MET25-Cub-RURA3. Before, pRS313-MET25-STE14-CubRURA3 was cut with ClaI, treated with the DNA-polymerase Klenow to generate a blunt side and finally cut with SalI to remove STE14.

\footnotetext{
${ }^{1}$ Mülhardt, Cornel: Der Experimentator Molekularbiologie/Genomics; 5. Auflage, München 2006, Seite 136
} 
For CUP1-Nui-ATG16, ATG16 was amplified with Nui-Atg16_BamHI_fwd and NuiAtg16_XhoI_rev. For CUP1-Nui-ATG27, ATG27 was amplified with NuiAtg27_BamHI_fwd and Nui-Atg27_XhoI_rev. All DNA fragments were cut with BamHI and XhoI and ligated into pRS314-CUP1-Nui.

For CUP1-Nui-ATG23, ATG23 was amplified with Nui-Atg23_BamHI_fwd and NuiAtg23_KpnI_rev, cut with BamHI and KpnI and ligated in pRS314-CUP1-Nui. Before, pRS314-CUP1-Nui-UBC6 was cut with BamHI and KpnI to remove UBC6. For CUP1-Nui-ATG12, ATG12 was amplified with Nui-Atg12_StuI_fwd and NuiAtg12_XhoI_rev, cut with StuI and ligated in pRS314-CUP1-Nui. Before, pRS314CUP1-Nui-UBC6 was cut with BamHI, treated with the DNA-polymerase Klenow to generate a blunt side and finally cut with XhoI to remove UBC6.

\subsubsection{Plasmid constructs for BiFC}

Plasmids for expression of proteins fused to one half of eYFP were kindly provided by Skarp et al. (2008). p426-ADH-C-YC and p426-ADH-N-YC allowed fusion of the C-terminal fragment of eYFP (amino acids 173 to 238) to the Crespectively N-terminus of a target protein. p425-ADH-N-YN allowed fusion of the N-terminal fragment of eYFP (amino acids 1 to 172 ) to the N-terminus of a target protein (Skarp et al., 2008).

For ADH-ATG21-C-YC, ATG21 was amplified with Atg21_C-YC_BamHI_fwd and Atg21_C-YC_SalI_rev, cut with BamHI and SalI and ligated in p426-ADH-C-YC. For ADH-N-YC-ATG21, ATG21 was amplified with Atg21_N-YC_BamHI_fwd and Atg21_N-YC_SalI_rev, cut with BamHI and SalI and ligated in p426-ADH-N-YC.

For ADH-ATG4-C-YC, ATG4 was amplified with Atg4_C-YC_EcoRI_fwd and Atg4_C-YC_SalI_rev, cut with EcoRI and SalI and ligated in p426-ADH-C-YC. For ADH-N-YC-ATG4, ATG4 was amplified with Atg4_N-YC_EcoRI_fwd and Atg4_NYC_SalI_rev, cut with EcoRI and SalI and ligated in p426-ADH-N-YC.

For ADH-N-YN-ATG8, ATG8 was amplified with Atg8_N-YN_BamHI_fwd and Atg8_N-YN_SalI_rev, cut with BamHI and SalI and ligated in p425-ADH-N-YN.

p416-MET25-C-YC was constructed by ligating a SalI and Xhol C-YC fragment from p426-ADH-C-YC into p416-MET25. Similarly, p415-MET25-N-YN was constructed by ligating a SpeI and BamHI N-YN fragment from p425-ADH-N-YN into p415-MET25. 
p416-MET25-ATG21-C-YC was constructed by ligating a BamHI and Xhol ATG21$C$-YC fragment from p426-ADH-ATG21-C-YC into p416-MET25. Similarly, p415MET25-N-YN-ATG8 was constructed by ligating a SpeI and HindIII N-YN ATG8 fragment from p425-ADH-N-YN-ATG8 into p415-MET25.

\subsubsection{Atg16 plasmid constructs}

For pRS313-CUP1-Atg16-HA, ATG16-HA was amplified with Atg16Cub_ClaI_fwd and Atg16HA_XhoI_rev using genomic DNA prepared from cAtg16-HA yeast cells. Similarly, for pRS313-CUP1-ATG161-57-HA and pRS313-CUP1-ATG161-119-HA, ATG161-57-HA and ATG161-119-HA were amplified with the same primer using genomic DNA prepared from cAtg16 1-57-HA respectively cAtg161-119-HA yeast cells. For pRS313-CUP1-ATG16 58-150-HA, ATG1658-150-HA was amplified with Atg16HA_58-150_ClaI_fwd and Atg16HA_XhoI_rev using genomic DNA prepared from cAtg16-HA yeast cells. The DNA fragments were cut with ClaI and XhoI and ligated into pRS313-CUP1.

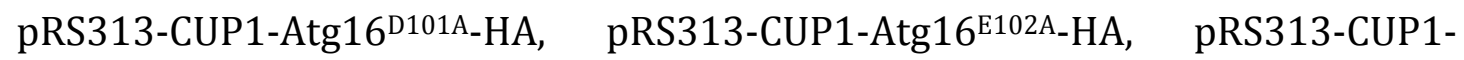

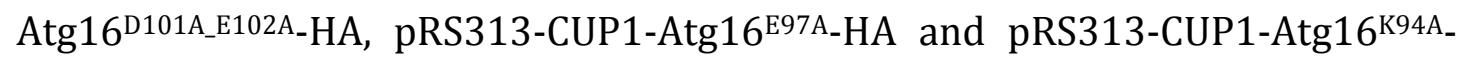
HA were constructed using the QuikChange II Site-Directed Mutagenesis Kit (Agilent) as described in the manufacturer's manual (see chapter 3.5.11). Point mutations were introduced using the respective primers: Atg16_D101A_fwd+_rev; Atg16_E102A_fwd+_rev; Atg16_DE_fwd+_rev; Atg16_E97A_fwd+_rev; Atg16_K94A_fwd+_rev.

For pGEX-4T3-GST-Atg16, ATG16 was amplified with GST-Atg16_BamHI_fwd and GST-Atg16_XhoI_rev using genomic yeast DNA. Similarly, ATG161-57 was amplified with GST-Atg16_BamHI_fwd and GST-Atg16 1-57_XhoI_rev, ATG16 1-119 was amplified with GST-Atg16_BamHI_fwd and GST-Atg161-119_XhoI_rev and ATG16 $58-150$ was amplified with GST-Atg1658-150_BamHI_fwd and GSTAtg16_XhoI_rev. DNA fragments were digested with BamHI and XhoI and ligated into pGEX-4T3. Point mutations were introduced as described above.

pRS313-Atg16 D101A-GFP, pRS313-Atg16 ${ }^{\text {E102A-GFP, pRS313-Atg16 }}$

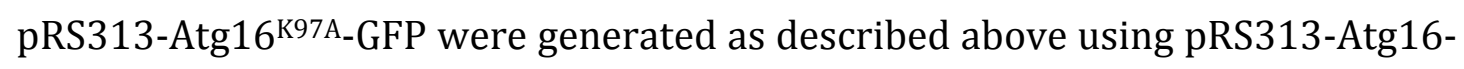
GFP as template. 


\subsubsection{Site-directed mutagenesis of plasmids}

To introduce point mutations in previously cloned genes, the QuikChange SiteDirected Mutagenesis Kit (Agilent) was used as described in the manufacturer's manual. For this, complementary primers containing the respective nucleotide exchange were generated. The successful introduction of the point mutation was verified by sequencing.

\subsubsection{Sequencing of DNA}

All new designed constructs were verified by sequencing using the Sanger method. For this purpose, $1 \mu \mathrm{l}$ of template plasmid DNA was mixed with $1 \mu \mathrm{l}$ sequencing mix (polymerase, dNTP mix with fluorescent dyes, $30 \mathrm{mM}$ magnesium chloride and buffer substances), 8 pmol oligonucleotides and $6 \mu \mathrm{l}$ $\mathrm{ddH}_{2} \mathrm{O}$. After the sequencing reaction $\left(25\right.$ cycles: $96^{\circ} \mathrm{C} 10 \mathrm{sec} ; 55^{\circ} \mathrm{C} 15 \mathrm{sec} ; 60^{\circ} \mathrm{C}$ $4 \mathrm{~min}$ ), the DNA was precipitated by adding $1 \mu \mathrm{l} 3 \mathrm{M}$ sodium acetate ( $\mathrm{pH}$ 5.2), $1 \mu \mathrm{l} 125 \mathrm{mM}$ EDTA and $50 \mu \mathrm{l}$ ethanol. The DNA was harvested by centrifugation (13200 rpm, 10 min, RT), washed with 70\% ethanol and dried. Finally, the DNA was resuspended in $15 \mu \mathrm{lHiDye}$ (formamide) and analyzed by the Department of Developmental Biology of the Georg-August-Universität Göttingen using the Genetic Analyzer 3100 (Applied Biosystems).

At the end of this study, sequencing was performed by GATC Biotech (Konstanz) as described in the manufacturer's instructions.

\subsubsection{Gene deletion using homologous recombination}

In this study, several deletion strains were performed using homologous recombination as described in Longtine et al. (1998). For this approach, a DNA fragment was amplified by PCR with special designed primers built up of a $45 \mathrm{bp}$ homologous sequence to the flanking region of the target gene followed by a $20 \mathrm{bp}$ homologous sequence of the selection gene (NatNT2 cassette). For amplification of the DNA fragment, the respective primers listed in Table 4 and pFA6a-NatNT2 (see Table 3) as plasmid template were used. The resulting DNA fragment, comprised of the selection gene, flanked by the homologous sequence to the up- and downstream region of the target gene, was transformed into the respective yeast strain using the high efficiency transformation protocol for yeast (see chapter 3.5.2). In principle, the similar sequence to the flanking 
regions of the target gene results in the exchange with the selection gene by homologous recombination. Potential clones were selected on medium containing NatNT2. The successful deletion of the target gene was verified by control PCR analysis using chromosomal DNA as template and primers binding to a region upstream of the target gene and within the selection gene respectively.

\subsection{Biochemical methods}

\subsubsection{SDS-Polyacrylamide-Gel-Electrophorese (SDS-PAGE)}

Discontinuous SDS-PAGE was performed as a standard method to separate proteins according to their electrophoretic mobility as described in Laemmli et al. (1970). For this purpose 5\% acrylamide stacking gels and, depending on the molecular size of the relevant proteins, $10-15 \%$ acrylamide separating gels were used.

Table 10: Contents of the separating gel

\begin{tabular}{llll}
\hline Separating Gel & $\mathbf{2 \times 1 0 \%}$ & $\mathbf{2 \times 1 2 \%}$ & $\mathbf{2} \times \mathbf{1 5 \%}$ \\
\hline $\mathbf{d d H}_{\mathbf{2}} \mathbf{0}$ & $3.9 \mathrm{ml}$ & $3.4 \mathrm{ml}$ & $2.4 \mathrm{ml}$ \\
$\mathbf{1 . 5 M}$ Tris (pH 8.8) & $2.5 \mathrm{ml}$ & $2.5 \mathrm{ml}$ & $2.5 \mathrm{ml}$ \\
Protogel & $3.5 \mathrm{ml}$ & $4 \mathrm{ml}$ & $5 \mathrm{ml}$ \\
$\mathbf{1 0 \%}$ SDS & $100 \mu \mathrm{l}$ & $100 \mu \mathrm{l}$ & $100 \mu \mathrm{l}$ \\
$\mathbf{1 0 \%}$ APS & $100 \mu \mathrm{l}$ & $100 \mu \mathrm{l}$ & $100 \mu \mathrm{l}$ \\
TEMED & $5 \mu \mathrm{l}$ & $5 \mu \mathrm{l}$ & $5 \mu \mathrm{l}$ \\
\hline
\end{tabular}

Table 11: Contents of the stacking gel

\begin{tabular}{ll}
\hline Stacking Gel & $\mathbf{2 x}$ \\
\hline $\mathbf{d d H}_{2} \mathbf{O}$ & $6.1 \mathrm{ml}$ \\
0.5M Tris (pH 6.8) & $2.5 \mathrm{ml}$ \\
Protogel & $1.3 \mathrm{ml}$ \\
$\mathbf{1 0 \%}$ SDS & $100 \mu \mathrm{l}$ \\
$\mathbf{1 0 \%}$ APS & $100 \mu \mathrm{l}$ \\
TEMED & $10 \mu \mathrm{l}$ \\
\hline
\end{tabular}

By default, $10 \mu \mathrm{l}$ of the protein sample and as a marker the Precision Plus Protein All Blue Standard (Bio-Rad) were loaded (molecular weights: 250 kDa, 150 kDa, 
$100 \mathrm{kDa}, 75 \mathrm{kDa}, 50 \mathrm{kDa}, 37.5 \mathrm{kDa}, 25 \mathrm{kDa}, 15 \mathrm{kDa} 10 \mathrm{kDa}$ ). Electrophoresis was performed in a Mini-PROTEAN III electrophoresis chamber from Bio-Rad at a voltage of $150 \mathrm{~V}$ until the bromphenolblue front reached the end of the gel. The separating gel was subsequently stained with colloidale Coomassie or used for Western Blot analysis (see chapter 3.6.2 and 3.6.3).

SDS-sample-buffer: $\quad 116 \mathrm{mM}$ Tris/HCl pH 6.8, $12 \%(\mathrm{w} / \mathrm{v}$ ) glycerol, 3.42\% (w/v) SDS, $0.004 \%$ bromphenolblue, $2 \% \beta$-mercaptoethanol

SDS-running-buffer: $\quad 200 \mathrm{mM}$ glycerol, $25 \mathrm{mM}$ Tris, $0.1 \%$ SDS

\subsubsection{Western Blot analysis}

Proteins separated by SDS-PAGE were transferred onto a PDVF membrane using custom-made semi dry blotting chambers or a wet blot Mini Trans-Blot Cell from Bio-Rad. For the semi dry blotting, the SDS-gel, a PVDF membrane and six thin Whatman filter papers were soaked with semi dry blotting buffer (25 mM Tris, $192 \mathrm{mM}$ glycine) and built up as shown in Figure 9. The transfer took place with $70 \mathrm{~mA}$ per gel for $90 \mathrm{~min}$ at room temperature.

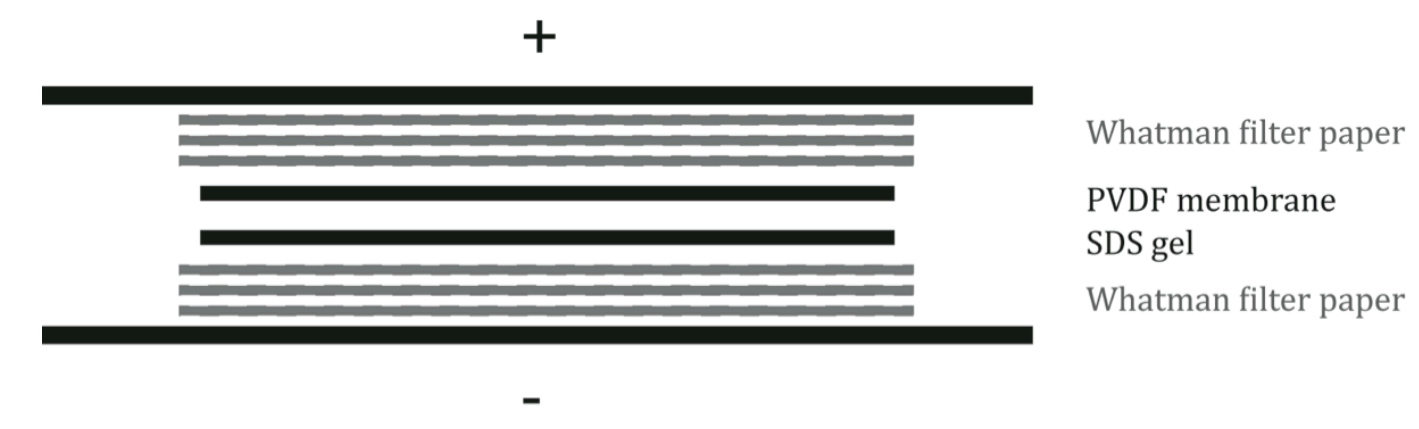

Figure 9: Scheme of semi dry Western Blot setting

For the wet blotting, the SDS-gel, a PDVF membrane, four thick Whatman filter papers and two filter pads were soaked with wet blotting buffer (192 mM glycin, $25 \mathrm{mM}$ Tris and 20\% methanol), assembled and inserted into the gel holder cassette as shown in Figure 10. The gel holder cassette was injected into the buffer tank filled with cold wet blotting buffer and an ice pack. The transfer was performed with $70 \mathrm{~mA}$ per gel for at least four hours up to over night at $4^{\circ} \mathrm{C}$. 


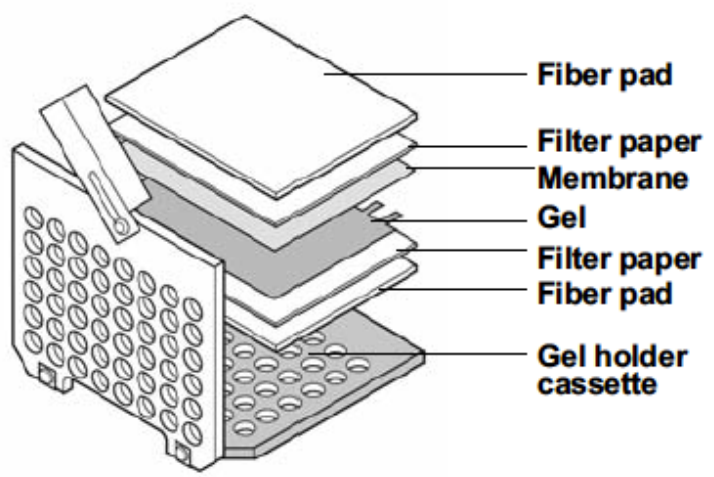

Figure 10: Scheme of Wet Western Blot settings (adapted from Mini Trans-Blot ${ }^{\circledR}$ Electrophoretic Transfer Cell Instruction Manual)

After successful blotting, the membrane was incubated in blocking buffer (TBST (20 mM Tris/HCl pH 7.6, $137 \mathrm{mM} \mathrm{NaCl}_{2}, 0.1 \%$ (w/v) Tween20) containing 10\% $(\mathrm{w} / \mathrm{v})$ skim milk powder) for at least one hour at room temperature or over night at $4^{\circ} \mathrm{C}$ to mask all unspecific binding sites on the membrane. After washing three times with $25 \mathrm{ml}$ TBST for $5 \mathrm{~min}$, the membrane was incubated with the primary antibody (see Table 5) for at least $2 \mathrm{~h}$ at room temperature or over night at $4^{\circ} \mathrm{C}$. After that, the membrane was washed again three times with $25 \mathrm{ml}$ TBST for $10 \mathrm{~min}$ and incubated for one hour at room temperature with the HRP coupled secondary antibody in $25 \mathrm{ml}$ TBST containing 1\% (w/v) skim milk powder (see Table 5). The membrane was washed again three times with TBST for $10 \mathrm{~min}$ and the signals were analyzed using the ECL ${ }^{\mathrm{TM}}$ system (USB) and LAS3000 (Fujifilm) according to the manufacturer's instructions. Quantitative statistics were performed using the AIDA Software, Version 4.06.116 (Raytest, 2005).

It is possible to strip off the antibodies of once immunodetected membranes and to incubate the membrane with another primary antibody. For this purpose, the membrane was first activated by methanol and after washing with TBST incubated in $10 \%(\mathrm{v} / \mathrm{v})$ acetic acid for $10 \mathrm{~min}$ at room temperature. After washing three times with TBST the membrane was incubated in $25 \mathrm{ml}$ blocking buffer and the cycle started again (see above).

\subsubsection{Colloidal Coomassie staining}

Unselective protein staining of SDS-gels was performed by colloidal Coomassie. The SDS-gel was first incubated for $1 \mathrm{~h}$ in $25 \mathrm{ml}$ fixating solution (40\% (v/v) ethanol, $10 \%(\mathrm{v} / \mathrm{v})$ acetic acid). After three washing steps with $\mathrm{ddH}_{2} \mathrm{O}$, proteins 
were incubated in staining solution $(20 \%$ (v/v) methanol, $0.1 \%(\mathrm{w} / \mathrm{v})$ Coomassie Brilliant Blue G250, 2\% (w/v) ortho-phosphoric acid, 10\% (w/v) ammonium sulfate) over night at room temperature. Background staining was removed by incubating in 1\% acetic acid until satisfaction. The Coomassie-stained gels were stored in $1 \%$ acetic acid or $\mathrm{ddH}_{2} \mathrm{O}$ at $4^{\circ} \mathrm{C}$.

\subsubsection{Determination of protein concentrations}

Protein concentrations were determined using a Bradford $\mathrm{DC}^{\mathrm{TM}}$ protein assay (Bio-Rad) according to the manufacturer's instructions.

\subsubsection{Alkaline lysis of yeast cells}

For a quick and harsh lysis of yeast cells the alkaline lysis procedure was used. For this purpose, $1-2 \mathrm{OD}_{600}$ of yeast cells was harvested by centrifugation (13200 rpm, $1 \mathrm{~min}, \mathrm{RT}$ ) and resuspended in $1 \mathrm{ml}$ ice-cold $\mathrm{dd}_{2} \mathrm{O}$ and $150 \mu \mathrm{l}$ lysis solution (1.85 M NaOH, 7.5\% (v/v) $\beta$-mercaptoethanol). After 10 min incubation on ice and repeated harsh mixing, $150 \mu \mathrm{l} 50 \%$ TCA solution was added and thoroughly mixed. After incubation on ice for $10 \mathrm{~min}$, the sample was centrifuged for $10 \mathrm{~min}$ at $13200 \mathrm{rpm}\left(4^{\circ} \mathrm{C}\right)$. The supernatant was removed and the pellet was washed twice with $200 \mu \mathrm{l}$ ice-cold acetone. Finally, the pellet was resuspended in 50 - $100 \mu \mathrm{l} 2$ x SDS-sample buffer (116 mM Tris/HCl pH 6.8, 12\% (w/v) glycerol, $3.42 \%(\mathrm{w} / \mathrm{v})$ SDS, $0.004 \%$ bromphenolblue, $2 \% \quad \beta$-mercaptoethanol) and analyzed by SDS-PAGE and Western Blotting.

\subsubsection{Cell lysis for detection of Atg8-PE}

For detection of Atg8-PE, cells were lysed as described in Suzuki et al. (2001). In detail, $60 \mathrm{OD}_{600}$ of growing yeast cells was harvested by centrifugation at $2000 \mathrm{rpm}$ for $5 \mathrm{~min}$ and resuspended in $500 \mu \mathrm{l}$ lysis buffer $(50 \mathrm{mM}$ Tris/HCl pH 7.5, $150 \mathrm{mM} \mathrm{NaCl}, 5 \mathrm{mM}$ EDTA, $5 \mathrm{mM}$ EGTA, $1 \mathrm{mM}$ PMSF, Complete ${ }^{\mathrm{TM}}$ (Roche), protease inhibitor). After addition of $200 \mu$ l glass beads, the cells were lysed by harsh mixing for $10 \mathrm{~min}$ at $4^{\circ} \mathrm{C}$. Afterwards, the cell lysate was cleared by centrifugating for $5 \mathrm{~min}$ at $3000 \mathrm{rpm}$ and $4^{\circ} \mathrm{C}$. $200 \mu \mathrm{l}$ of the supernatant was transferred into a new reaction tube and, after addition of $40 \mu \mathrm{l} 6 \mathrm{x}$ Laemmli, boiled at $95^{\circ} \mathrm{C}$ for $5 \mathrm{~min}$. For separating Atg8 and Atg8-PE, the samples were 
separated on a 15\% SDS-PAGE containing $6 \mathrm{M}$ urea, subjected to immunoblotting and analyzed using an anti-Atg8 antibody (see Table 5).

\subsubsection{Preparation of whole cell extracts - the mild cell lysis procedure}

For gelfiltration chromatography, sorbitol density gradient centrifugation and GFP-TRAP ${ }^{\circledR}$ analysis, it was needed to prepare crude yeast cell lysate in a special mild cell lysis procedure. For this purpose, the cells were first converted into spheroblasts and subsequently mechanically lysed using a glass/ceramic homogenizer. In detail, $500 \mathrm{OD}_{600}$ yeast cells was harvested by centrifugation at $2000 \mathrm{rpm}$ for $5 \mathrm{~min}$. The cells were once washed with $30 \mathrm{ml} 10 \mathrm{mM} \mathrm{NaN}_{3}$, resuspended in $5 \mathrm{ml}$ SP-buffer (1.4 $\mathrm{M}$ sorbitol, $50 \mathrm{mM} \mathrm{KH}_{2} \mathrm{PO}_{4} \mathrm{pH} 7.5,10 \mathrm{mM}$ $\mathrm{NaN}_{3}, 40 \mathrm{mM} \beta$-mercaptoethanol) containing $1.5 \mathrm{mg}$ zymolyase T100 (the zymolyase has been shaken in SP-buffer for $30 \mathrm{~min}$ at $30^{\circ} \mathrm{C}$ before) and incubated for $40 \mathrm{~min}$ at $30 \mathrm{~min}$ in a water bath under weak shaking. The generated spheroblasts were harvested by centrifugation at $1000 \mathrm{xg}$ for $10 \mathrm{~min}$ at $4^{\circ} \mathrm{C}$ and washed with $5 \mathrm{ml}$ SP-buffer. SP-buffer was added in $1 \mathrm{ml}$ steps and the spheroblasts were carefully resuspended using a rounded glass stick. After centrifugation at $2000 \mathrm{xg}$ for $10 \mathrm{~min}$ at $4^{\circ} \mathrm{C}$, the spheroblasts were resuspended in $500 \mu \mathrm{l}$ of the particular lysis buffer (see below) and transferred into an icecooled glass/ceramic homogenizer. The cells were lysed with 30 pushes and the cell lysate was transferred into a new reaction cup. The homogenizer was rinsed with $500 \mu \mathrm{l}$ lysis buffer, which was added to the cell lysate. If not stated otherwise, $0.5 \%$ Triton X-100 was added and the mix was incubated for $5 \mathrm{~min}$ at $4^{\circ} \mathrm{C}$. Cell debris was removed by centrifugation at $3000 \mathrm{xg}$ for $10 \mathrm{~min}$ at $4^{\circ} \mathrm{C}$ and the remaining crude cell lysate was used for the particular application mentioned above. The volume of the SP- and lysis buffer was adapted to the particular yeast amount if the approach was scaled up or down.

\subsubsection{GFP-TRAP ${ }^{\circledR}$}

For the GFP-TRAP ${ }^{\circledR}, 350 \mathrm{OD}_{600}$ yeast cells was harvested, treated and lysed in $350 \mu \mathrm{l}$ TRAP lysis buffer (1 x PBS pH 7.4, $0.2 \mathrm{M}$ sorbitol, $5 \mathrm{mM} \mathrm{MgCl}_{2}, 1 \mathrm{mM}$ PMSF, Complete ${ }^{\mathrm{TM}}$ (Roche), protease inhibitors) as described in chapter 3.6.7. For immunoblot analysis, $25 \mu \mathrm{l}$ of the cell lysate was diluted with $25 \mu \mathrm{l}$ $6 \times$ Laemmli buffer containing 6\% $\beta$-mercaptoethanol and boiled for $10 \mathrm{~min}$ at 
$95^{\circ} \mathrm{C}$ (refer to as input). The remaining crude cell lysate was supplemented with $10 \mu \mathrm{l}$ GFP-TRAP ${ }^{\circledR}$ bead slurry, which was washed twice with $100 \mu$ PBS before $\left(2700 \mathrm{xg}, 2 \mathrm{~min}, 4^{\circ} \mathrm{C}\right)$. After incubation for $2-4 \mathrm{~h}$ at $4^{\circ} \mathrm{C}$ under constant inverting, the beads were harvested by centrifugation at $2000 \mathrm{xg}$ for $2 \mathrm{~min}$ at $4^{\circ} \mathrm{C} .25 \mu \mathrm{l}$ of the supernatant was diluted with $25 \mu \mathrm{l} 6 \times$ Laemmli buffer containing $6 \% \beta$-mercaptoethanol and boiled for $10 \mathrm{~min}$ at $95^{\circ} \mathrm{C}$ (refer to as supernatant). The beads were washed once with $1 \mathrm{ml}$ and afterwards 3 times with $0.5 \mathrm{ml}$ lysis buffer $\left(2000 \mathrm{xg}, 2 \mathrm{~min}, 4^{\circ} \mathrm{C}\right)$ and finally eluted in $50 \mu \mathrm{l} 2 \mathrm{x}$ Laemmli buffer containing $2 \% \beta$-mercaptoethanol by boiling for $10 \mathrm{~min}$ at $95^{\circ} \mathrm{C}$ (refer to as bound). The samples were subjected to Wet Western Blot analysis as described in chapter 3.6.2.

\subsubsection{Gelfiltration chromatography}

For analysis of the high molecular weight Atg21 complex, yeast cells were grown in selection medium to mid-log phase. $1000 \mathrm{OD}_{600}$ log cells were prepared and lysed in $1 \mathrm{ml}$ GF lysis buffer (0.8 M sorbitol, $10 \mathrm{mM}$ MOPS pH 7.2, $1 \mathrm{mM}$ EDTA, $2 \mathrm{mM}$ PMSF, Complete ${ }^{\mathrm{TM}}$ (Roche), protease inhibitors) as described in chapter 3.6.7. After preclearing for $10 \mathrm{~min}$ at $500 \mathrm{xg}$ and $4^{\circ} \mathrm{C}$, the cell lysate was centrifuged at $49000 \mathrm{rpm}$ for $30 \mathrm{~min}$ at $4^{\circ} \mathrm{C}$ (Beckman ultracentrifuge, Rotor TLA-100.3). The resultant supernatant was separated by size exclusion chromatography on a Superose ${ }^{\mathrm{TM}} 6$ 10/300 GL column (Amersham Pharmacia Biotech, Schweden), which was equilibrated with lysis buffer before. The elution from the column was carried out at a flow rate of $0.5 \mathrm{ml} / \mathrm{min}$ and $0.8 \mathrm{ml}$ fractions were collected. The fractionated cell lysate was precipitated by adding $80 \mu \mathrm{l}$ $100 \%$ TCA and $10 \mathrm{~min}$ storage on ice. After centrifugation for $10 \mathrm{~min}$ at $13200 \mathrm{rpm}$ at $4^{\circ} \mathrm{C}$, the pellet was washed twice with $200 \mu \mathrm{l}$ acetone. The pellet was dried at $37^{\circ} \mathrm{C}$ for $10 \mathrm{~min}$ and finally resuspended in $50 \mu \mathrm{l}$ Laemmli buffer containing 2\% $\beta$-ME and analyzed by SDS-PAGE and Wet Western Blotting as described in chapter 3.6.2. To determine the size of potential high molecular weight complexes, marker proteins (ApeI, PGK1 and CPY1) with well known molecular masses were detected by immunoblot analysis using anti-ApeI, -PGK1 and -CPY1 antibodies (see Table 5). 


\subsubsection{Density gradient centrifugation}

Density gradient centrifugation was used to further analyze the high molecular weight Atg21 complex. Cells expressing pMET25-mCherry or pMET25-mCherryAtg21 were grown in the absence of methionine to mid-log phase. $500 \mathrm{OD}_{600}$ of cells was harvested, treated and lysed in $500 \mu$ lysis buffer (10 mM MOPS pH 7.2, $0.2 \mathrm{M}$ sorbitol, $1 \mathrm{mM}$ EDTA, $2 \mathrm{mM}$ PMSF, Complete ${ }^{\mathrm{TM}}$, protease inhibitor) as described in chapter 3.6.7. After preclearing of the crude cell extract $(10 \mathrm{~min}$, $500 \mathrm{xg}, 4^{\circ} \mathrm{C}$ ), the supernatant was ultracentrifuged for $30 \mathrm{~min}$ at $100000 \mathrm{xg}$ and $4^{\circ} \mathrm{C}$ (Beckman ultracentrifuge, Rotor TLA-100.3). The resulting supernatant was loaded on the top of a glycerol density gradient (per step $450 \mu \mathrm{l} 50 \%, 40 \%, 30 \%$, $20 \%$ glycerol) or sorbitol density gradient (per step $450 \mu \mathrm{l} 2.5 \mathrm{M}, 2 \mathrm{M}, 1.5 \mathrm{M}$, $0.9 \mathrm{M}$ sorbitol). After $4 \mathrm{~h}$ of ultracentrifugation at $259000 \mathrm{xg}$ and $4^{\circ} \mathrm{C}$ (Beckman ultracentrifugation, Rotor TLS-55), ten fractions each $200 \mu$ l were collected and precipitated with $20 \mu \mathrm{l} 100 \%$ TCA. The sediments were washed two times with $200 \mu \mathrm{l}$ acetone, resuspended in $100 \mu \mathrm{l} 2 \times$ Laemmli buffer and subjected to immunoblotting.

Table 12: Densities of the glycerol or sorbitol solutions used in density gradient centrifugation

\begin{tabular}{lcc}
\hline density $[\mathrm{g} / \mathrm{ml}]$ & Glycerol & Sorbitol \\
\hline 1.145 & $50 \%$ & $2.5 \mathrm{M}$ \\
1.111 & $40 \%$ & $2 \mathrm{M}$ \\
1.085 & $30 \%$ & $1.5 \mathrm{M}$ \\
1.049 & $20 \%$ & $0.9 \mathrm{M}$ \\
\hline
\end{tabular}

\subsubsection{Pull down of His-SUMO-Atg21}

\subsubsection{Purification of recombinant 6xHis-SUMO-Atg21 and 6xHis-SUMO}

Liquid precultures were inoculated with E. coli BL21 (DE3) pLysS cells expressing 6xHis-SUMO-Atg21 or 6xHis-SUMO alone from either an agar plate using a sterile toothpick or from a short-term culture. The cells were shaken at $37^{\circ} \mathrm{C}$ over night $(12-14 \mathrm{~h})$ at $220 \mathrm{rpm}$. At the next morning the cells were diluted to an $\mathrm{OD}_{600}$ of 0.2 and shaken for $90 \mathrm{~min}$ at $30^{\circ} \mathrm{C}$ and $220 \mathrm{rpm}$. To induct the expression of 6xHis-SUMO-Atg21 or 6xHis-SUMO, $0.2 \mathrm{mM}$ IPTG and $1 \mathrm{mM}$ PMSF were added. The cells were grown at $30^{\circ} \mathrm{C}$ for $4.5-5 \mathrm{~h}$, harvested by centrifugation (5 min, $5000 \mathrm{rpm}, \mathrm{RT}$ ), washed with $10 \mathrm{ml} 1 \times \mathrm{PBS}(\mathrm{pH} \mathrm{7.4)}$ and 
finally frozen in liquid nitrogen. The cell pellets were stored at $-80^{\circ} \mathrm{C}$ until use. The frozen cell pellets were resuspended in ice-cold lysis buffer (1 x PBS pH 7.4, $2 \mathrm{mM} \mathrm{MgCl}$, protease inhibitors (Sigma), $1 \mu \mathrm{l}$ benzonase) and stored on ice for $10 \mathrm{~min}$. For immunoblot analysis, $15 \mu \mathrm{l}$ of the cell lysate was diluted with $15 \mu \mathrm{l}$ $6 \mathrm{x}$ Laemmli buffer containing $50 \mathrm{mM}$ DTT and boiled for $10 \mathrm{~min}$ at $72^{\circ} \mathrm{C}$ (refer to as E. coli L). After addition of $1 \%(\mathrm{v} / \mathrm{v})$ Triton X-100, the unsoluble parts were spun out for $10 \mathrm{~min}$ at $10000 \mathrm{rpm}$ and $4^{\circ} \mathrm{C}$. For immunoblot analysis, $15 \mu \mathrm{l}$ of the supernatant was diluted with $15 \mu \mathrm{l} 6$ x Laemmli buffer containing $50 \mathrm{mM}$ DTT and boiled for $10 \mathrm{~min}$ at $72^{\circ} \mathrm{C}$ (refer to as E. coli $\mathrm{T}$ ). To couple 6xHis-SUMO-Atg21 to an agarose matrix via its His-tag, the remaining supernatant was added to $100 \mu \mathrm{l} 50 \% \mathrm{Ni}$-NTA beads slurry (Qiagen, Hilden), which was washed twice with

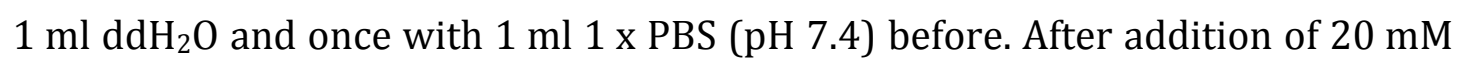
imidazole (fresh prepared) to reduce unspecific background binding, the mix was incubated for $45 \mathrm{~min}$ at $4^{\circ} \mathrm{C}$ under constant shaking. The beads were harvested by centrifugation ( $5 \mathrm{~min}, 500 \mathrm{xg}, 4^{\circ} \mathrm{C}$ ), washed four times with $500 \mu \mathrm{l}$ wash buffer (1 x PBS pH 7.4, $20 \mathrm{mM}$ imidazole) and finally stored in $500 \mu \mathrm{l} 1 \mathrm{x}$ PBS (pH 7.4) on ice until use.

\subsubsection{6xHis-SUMO-Atg21 pull down with crude yeast cell extract}

$50 \mathrm{OD}_{600}$ yeast cells was harvested by centrifugation $\left(2000 \mathrm{rpm}, 5 \mathrm{~min}, 4^{\circ} \mathrm{C}\right.$ ), washed with $5 \mathrm{ml} 1 \mathrm{x}$ PBS (pH 7.4) and subsequently resuspended in $1 \mathrm{ml}$ lysis buffer (1 x PBS pH 7.4, 5 mM EDTA, 0.5\% (v/v) Triton X-100, $1 \mathrm{mM} \mathrm{PMSF,}$ Complete $^{\mathrm{TM}}$ (Roche), protease inhibitors). After addition of $0.2 \mathrm{ml}$ glass beads, the cells were mixed harsh for $20 \mathrm{~min}$ at $4^{\circ} \mathrm{C}$ followed by a preclearing centrifugation step at $3000 \mathrm{rpm}$ and $4^{\circ} \mathrm{C}$ for $5 \mathrm{~min}$. For immunoblot analysis, $50 \mu \mathrm{l}$ of the supernatant was diluted with $10 \mu \mathrm{l} 6 \mathrm{x}$ Laemmli buffer containing $50 \mathrm{mM}$ DTT and boiled for $10 \mathrm{~min}$ at $72^{\circ} \mathrm{C}$ (refer to as yeast input). The remaining supernatant was given to the prepared Ni-NTA-6xHis-SUMO-Atg21 or -SUMO beads (see chapter 3.6.11.1). The mix was incubated $2-4 \mathrm{~h}$ at $4^{\circ} \mathrm{C}$ under constant shaking. After extensive washing with lysis buffer, bound proteins were eluted with $50 \mu \mathrm{l}$ Laemmli buffer containing $50 \mathrm{mM}$ DTT, boiled for $10 \mathrm{~min}$ at $72^{\circ} \mathrm{C}$ (refer to as bound) and analyzed by $12 \%$ SDS-PAGE and following Wet Western Blot analysis (see chapter 3.6.2). 


\subsubsection{Purification of GST-Atg16}

To test, if Atg21 and Atg16 interact directly with each other, recombinant expressed GST-Atg16 was incubated with recombinant 6xHis-SUMO-Atg21. For this purpose, GST-Atg16 was expressed and purified analogous to 6xHis-SUMOAtg21 (see chapter 3.6.11.1) without imidazole. Furthermore, $100 \mu \mathrm{l}$ of a $50 \%$ Glutathione Sepharose ${ }^{\mathrm{TM}}$ 4B (GE healthcare) was used for coupling. The bound GST-Atg16 constructs were eluted with $300 \mu$ l glutathione elution buffer $(10 \mathrm{mM}$ reduced glutathione, $50 \mathrm{mM}$ Tris/HCl pH 8.0) in a three-step procedure. For this purpose, $100 \mu \mathrm{l}$ of the elution buffer was added to the GST column and incubated for $10 \mathrm{~min}$ at room temperature under constant mixing. After sedimentation of the beads ( $3 \mathrm{~min}, 500 \mathrm{xg}$, RT), the supernatant containing eluted GST-Atg16 was collected and the elution procedure was repeated two times. The protein concentration of the eluted GST-Atg16 constructs was determined using Bradford assay (see chapter 3.6.4). The purified GST-Atg16 variants were used for the direct SUMO-Atg21 pull down (see chapter 3.6.11.4).

\subsubsection{6xHis-SUMO-Atg21 pull down with recombinant proteins}

To determine a direct interaction of Atg21 and Atg16, purified recombinant 6xHis-SUMO-Atg21 was incubated with purified recombinant GST-Atg16. For this purpose, purified GST-Atg16 variants (see chapter 3.6.11.3) were diluted with binding buffer (20 mM Na-P-buffer pH 7.4, $150 \mathrm{mM} \mathrm{NaCl}, 1 \mathrm{mM}$ EDTA, 0.2\% (v/v) Triton X-100, 1 mM PMSF, 1 x Complete (Roche), protease inhibitors) to a concentration of $10 \mu \mathrm{M}$ in $500 \mu \mathrm{l}$. For immunoblot analysis, $20 \mu \mathrm{l}$ of the preparation was diluted with $10 \mu \mathrm{l} 6$ x Laemmli buffer containing 50 mM DTT and boiled for $10 \mathrm{~min}$ at $72^{\circ} \mathrm{C}$ (refer to as input). The remaining GST-Atg16 was added to the purified and immobilized 6xHis-SUMO-Atg21 (see chapter 3.6.11.1), which was equilibrated with binding buffer before. After incubation for $2 \mathrm{~h}$ at $4^{\circ} \mathrm{C}$ under constant mixing, the beads were sedimented $\left(5 \mathrm{~min}, 500 \times \mathrm{g}, 4^{\circ} \mathrm{C}\right)$ and washed twice with $500 \mu \mathrm{l}$ binding buffer and once with $500 \mu \mathrm{l}$ wash buffer (20 mM Na-P-buffer pH 7.4, 150 mM NaCl, 1.5 mM EDTA, 0.2\% (v/v) Triton X100, 1 mM PMSF, Complete ${ }^{\mathrm{TM}}$ (Roche), protease inhibitors). The bound proteins were eluted with $50 \mu \mathrm{l}$ Laemmli buffer containing $50 \mathrm{mM}$ DTT, boiled for $10 \mathrm{~min}$ 
at $72{ }^{\circ} \mathrm{C}$ (refer to as bound) and analyzed by $12 \%$ SDS-PAGE and following Wet Western Blot analysis (see chapter 3.6.2).

\subsubsection{Mass spectrometry analysis}

Mass spectrometry analysis was performed by the Bioanalytical Mass Spectrometry Group of Prof. Henning Urlaub (Bioanalytics, Department of Clinical Chemistry, University Göttingen).

\subsubsection{Materials}

Chemicals used were from Sigma-Aldrich, Taufkirchen. Solvents were obtained from Merck KgaA (Darmstadt) in Lichrosolv quality. Porcine trypsin was obtained from Serva Electrophoresis (Heidelberg). Chromatography materials were obtained from Dr. Maisch (Ammerbuch-Entringen).

\subsubsection{In-gel digestion}

Gel spots were washed with water, reduced with DTT $(10 \mathrm{mM}$ in $100 \mathrm{mM}$ $\left.\mathrm{NH}_{4} \mathrm{HCO}_{3}, 50 \mathrm{~min}, 56^{\circ} \mathrm{C}\right)$ and alkylated with iodoacetamide (55 mM in $100 \mathrm{mM}$ $\mathrm{NH}_{4} \mathrm{HCO}_{3}, 20 \mathrm{~min}$, room temperature, dark). In between sample handling steps, the gel spots were washed with acetonitrile for 15 min and taken to dryness in a Speedvac at $35^{\circ} \mathrm{C}$ (Savant Model SPD111V, Thermo Scientific, Dreieich) to remove excess solvent and improve uptake of reagent solution. Gel spots were rehydrated with porcine trypsin $\left(12.5 \mathrm{ng} / \mu \mathrm{l}\right.$ in $\left.50 \mathrm{mM} \mathrm{NH}_{4} \mathrm{HCO}_{3}, 5 \mathrm{mM} \mathrm{CaCl}_{2}\right)$, $50 \mathrm{mM} \mathrm{NH}_{4} \mathrm{HCO}_{3}$ added as supernatant and digested over night at $37^{\circ} \mathrm{C}$.

\subsubsection{Peptide extraction}

Following digestion, $10 \mu \mathrm{l}$ of water was added and the gel pieces were incubated at $37^{\circ} \mathrm{C}$ for $15 \mathrm{~min}$. After a short centrifugation step, $80 \mu \mathrm{l}$ acetonitrile was added and the samples again incubated at $37^{\circ} \mathrm{C}$ for $15 \mathrm{~min}$. The supernatant was removed and transferred to an Eppendorf vial.

$65 \mu \mathrm{l}$ of $5 \%$ formic acid was added to the gel pieces, vortexed and incubated at $37^{\circ} \mathrm{C}$ for $15 \mathrm{~min} .65 \mu \mathrm{l}$ of acetonitrile was added and again incubated at $37^{\circ} \mathrm{C}$ for 15 min. After a short centrifuge step, excess solvent was removed and pooled with the supernatant from the first extraction step. The combined supernatants were taken to dryness in a Speedvac at $35^{\circ} \mathrm{C}$ and stored at $-20^{\circ} \mathrm{C}$ until analysis. 


\subsubsection{Mass spectrometric analysis}

The resulting peptide mixtures were concentrated on a Reversed Phase-C18 precolumn (0.15 mm ID x 20 mm self-packed with Reprosil-Pur 120 C18-AQ $3 \mu \mathrm{m}$ material) and separated by Reversed Phase-C18 nanoflow chromatography (0.075 mm ID x 200 mm Picofrit column (New Objective, Woburn, MA/USA) selfpacked with Reprosil-Pur 120 C18-AQ $3 \mu \mathrm{m}$ material) using a 22 min linear gradient (5-35\% acetronitrile vs. $0.1 \%$ formic acid, $240 \mathrm{nl} / \mathrm{min}$ ) on an EASY nLC-1000 system (Thermo Scientific, Dreieich). The eluent was analyzed using a Top10 method in Data Dependent Acquisition mode on a Q Exactive high resolution mass spectrometry system (Thermo Scientific, Dreieich) operated under Tune 2.2 using HCD fragmentation, with a Normalized Collision Energy of 25\%. Peak lists were generated using Raw2MSM v1.10 software (MPI for Biochemistry, Martinsried).

\subsubsection{Database Searching}

Tandem mass spectra were extracted by Raw2MSM software version 1.7 including charge state deconvolution and deisotoping. All MS/MS samples were analyzed using Mascot (Matrix Science, London, UK; version 2.4.1). Mascot was set up to search the SwissProt 2012_11x database (selected for Saccharomyces cerevisiae, 538585 sequences, 191240774 residues) assuming the digestion enzyme trypsin. Mascot was searched with a fragment ion mass tolerance of 0.020 Da and a parent ion tolerance of 10.0 PPM. Carbamidomethylation of cysteine was specified in Mascot as a fixed modification. Oxidation of methionine was specified in Mascot as a variable modification. 


\section{Results}

\subsection{Aim of the study}

Atg21 and its homologues Atg18 and Hsv2, belong to a family of PI3P effectors involved in autophagy. They contain seven WD40 repeats, which form a seven bladed $\beta$-propeller scaffold (Baskaran et al., 2012; Krick et al., 2012; Watanabe et al., 2012). These proteins are known to serve as protein interaction platforms (Xu and Min, 2011). Atg21 has two lipid-binding pockets, which preferentially bind to PtdIns(3)P and PtdIns(3,5) $\mathrm{P}_{2}$ and mediate its peripheral membrane association (Baskaran et al., 2012; Krick et al., 2012; Watanabe et al., 2012). While macroautophagy is only significantly reduced in the absence of Atg21, Atg21 is required for selective types of autophagy like the Cvt-pathway. Atg21 is involved in the biogenesis of the Cvt vesicles (Meiling-Wesse et al., 2004). In detail, Atg21 is required for the efficient lipidation of Atg8 and the recruitment of Atg8 and Atg5 to the pre-autophagosomal structure (PAS) (Meiling-Wesse et al., 2004; Stromhaug et al., 2004). Atg21 has been detected at endosomal compartments and at vertices of vacuolar junctions (Krick et al., 2008a; MeilingWesse et al., 2004). Despite its fundamental role in Cvt vesicle formation and the PAS recruitment of other autophagy-related proteins, up to now Atg21 has not been detected at the PAS itself (Stromhaug et al., 2004). Detection of its localization at the PAS and the identification of potential interaction partner of Atg21 will elucidate its molecular function and its role in the autophagic process.

\subsection{Localization studies of Atg21}

\subsubsection{Part of Atg21-YFP was detected at the PAS}

The PAS is defined as the site within a cell, where almost all of the autophagy related proteins colocalize and where the formation of the autophagosome is initiated. Although atg21د cells fail to form Cvt vesicles, Atg21 has not been detected at the PAS so far. To get a closer look, Atg21-YFP, expressed from its own promoter (Meiling-Wesse et al., 2004), was coexpressed with the PAS marker aminopeptidase I-RFP (ApeI-RFP) in atg21 $\Delta$ cells and analyzed by direct fluorescence microscopy. Since Atg21 is required for the Cvt-pathway, that acts 
under growing conditions, cells were imaged under these conditions. In growing atg21 cells, Atg21-YFP formed one to six perivacuolar puncta per cell (Figure 11). Some of them have been reported to colocalize with the late endosomal marker mRFP-FYVE (Krick et al., 2008a). For further localization analysis, the percentage of cells was determined, that exhibit an Atg21-YFP signal and an additional perivacuolar ApeI-RFP dot (PAS rate). Furthermore, the percentage of ApeI-RFP dots was determined that colocalized with Atg21-YFP (colocalization rate). Interestingly, 49\% of the ApeI-RFP dots colocalized with Atg21-YFP suggesting that part of Atg21-YFP was indeed localized at the PAS. As a negative control, atg14A cells were utilized. Atg14 directs the Vps34 kinase complex I to the PAS, where it is required to generate the PAS-specific PI3P pool (Obara et al., 2006). Atg21 is peripherally membrane associated by binding to PI3P and $\mathrm{PI}(3,5) \mathrm{P}_{2}$ (Krick et al., 2006). In the absence of Atg14, and thus PI3P from the PAS, Atg21 should be absent from the PAS as it was shown earlier for its homologue Atg18 (Suzuki et al., 2007). Indeed, the colocalization of Atg21-YFP with the PAS marker ApeI-RFP was significantly reduced to only $22 \%$ in atg14A $\log$ cells.

For the homologue Atg18 an additional interaction with Atg2 is needed for proper membrane association at the PAS (Obara et al., 2008b; Rieter et al., 2013). To determine, which protein partner is responsible for proper PAS localization of Atg21, the colocalization rate of Atg21-YFP and the PAS marker ApeI-RFP was analyzed in several deletion strains by fluorescence microscopy as described above (Figure 11 + Figure 12). 
Results

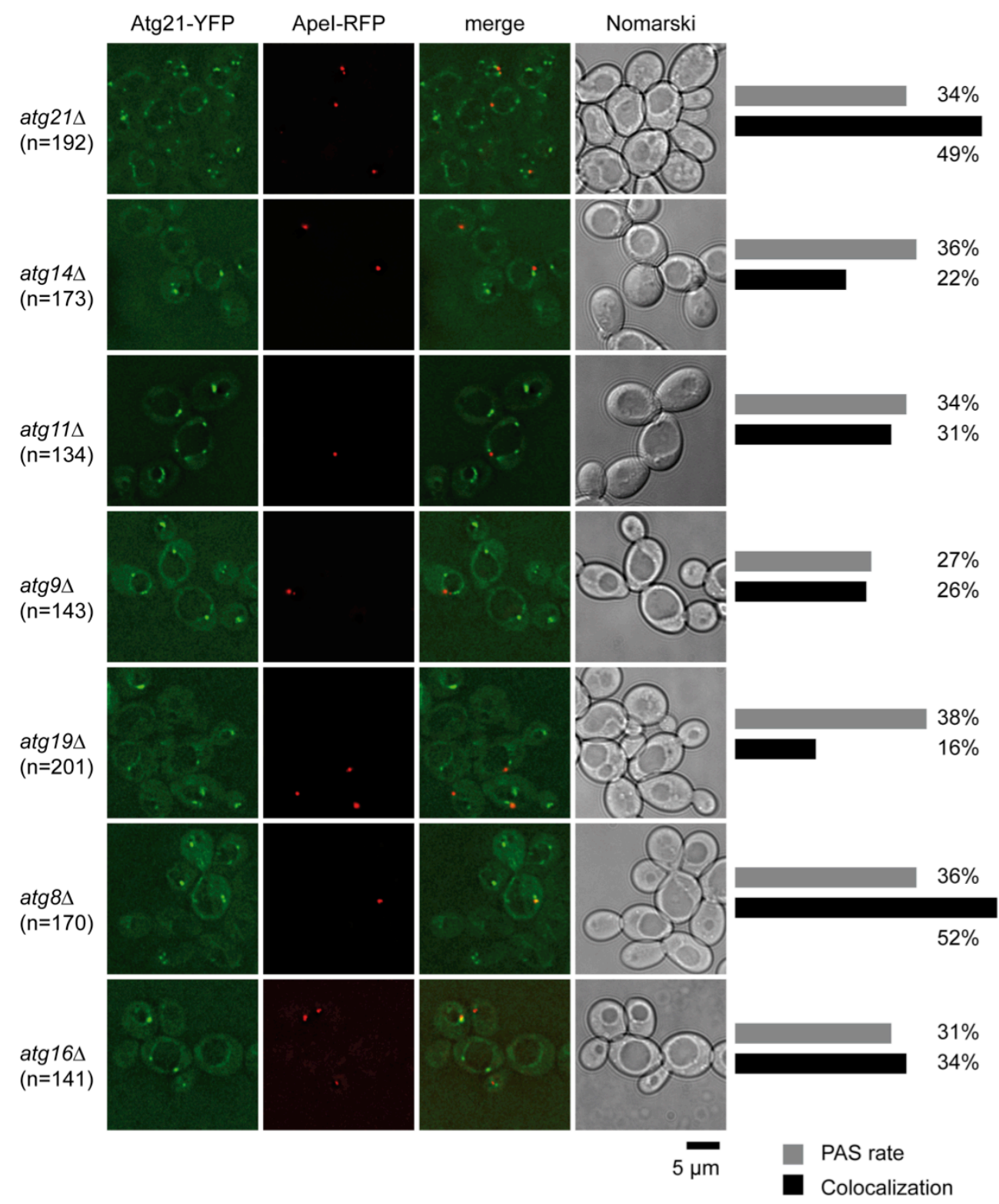

\section{Figure 11: Part of Atg21 is localized at the PAS}

The indicated strains expressing Atg21-YFP and the PAS marker ApeI-RFP were grown to log phase in selective medium at $30^{\circ} \mathrm{C}$ and visualized by direct fluorescence microscopy using a DeltaVision Deconvolution fluorescence microscope equipped with FITC and TRITC filter sets. Pictures were deconvoluted using SoftWoRx (Applied Precision) software. For each strain, the PAS rate and the Colocalization rate of Atg21-YFP and ApeI-RFP were determined. The PAS rate indicated, how many of the cells with an Atg21-YFP signal showed a perivacuolar ApeI-RFP dot. The Colocalization rate indicated, how many of those dots overlapped with Atg21-YFP. The number of evaluated cells ( $\mathrm{n}$ ) is indicated.

In the absence of Atg11, a scaffold protein crucial for PAS formation under growing condition, the colocalization rate of Atg21-YFP and ApeI-RFP was 
slightly reduced to $34 \%$ compared to the wildtype (atg21 $=49 \%$ ). The loss of the transmembrane protein Atg9, which delivers at least part of the membrane to the forming autophagosome or the loss of the Cvt cargo receptor Atg19, led to a more significant reduction of the colocalization rate to $26 \%$ and $16 \%$, respectively. In the absence of Atg16, member of the E3 enzyme complex required for Atg8 lipidation, the Atg18-interacting protein Atg2, or the membrane protein Atg27, the colocalization rate was slightly reduced to $34 \%$ or $38 \%$, respectively (Figure 11+Figure 12). In contrast, neither Atg8 nor Atg23 showed an influence on the PAS localization of Atg21-YFP (52\% respectively $48 \%)$.

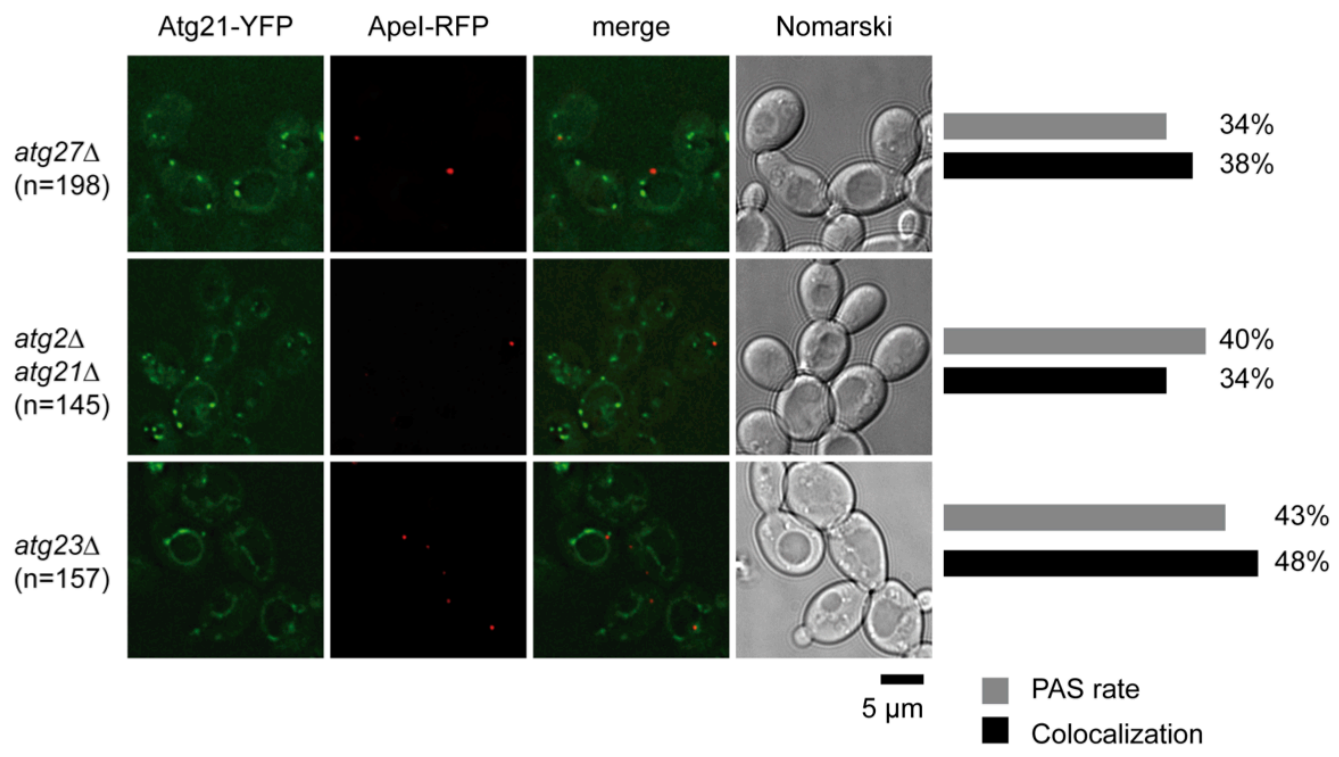

Figure 12: Additional analysis of the PAS localization of Atg21-YFP

The indicated strains expressing Atg21-YFP and the PAS marker ApeI-RFP were grown to log phase in selective medium at $30^{\circ} \mathrm{C}$ and visualized by direct fluorescence microscopy using a DeltaVision Deconvolution fluorescence microscope equipped with FITC and TRITC filter sets. Pictures were deconvoluted using SoftWoRx (Applied Precision) software. For each strain, the PAS rate and the colocalization of Atg21-YFP and ApeI-RFP were determined. PAS rate indicated, how many of the cells with an Atg21-YFP signal showed a perivacuolar ApeI-RFP dot. Colocalization indicated, how many of these dots overlapped with Atg21-YFP. The number of evaluated cells (n) is indicated.

Furthermore, none of the tested proteins had an influence on the overall punctate appearance of Atg21-YFP.

In further approaches, a pMET25-mCherry-Atg21 construct was used. To exclude overexpression artefacts caused by the expression from the inducible MET25 promoter, the localization of mCherry-Atg21 was analyzed by direct fluorescence 
microscopy as described above. $0.3 \mathrm{mM}$ methionine was added to the selective medium to induce endogenous expression level (Krick et al., 2008a). In growing atg21 cells expressing mCherry-Atg21 and the PAS marker ApeI-YFP, 46\% of the ApeI-YFP dots colocalized with mCherry-Atg21, whereas the colocalization rate was significantly reduced to only $23 \%$ in growing $\operatorname{atg} 14 \Delta$ cells (Figure $13 \mathrm{~A}$ ). Both colocalization rates correlated with the respective rates from $\operatorname{atg} 21 \Delta$ and atg14D cells expressing Atg21-YFP from its endogenous promoter and ApeI-RFP, indicating that mCherry-Atg21 behaves like Atg21-YFP in living cells.

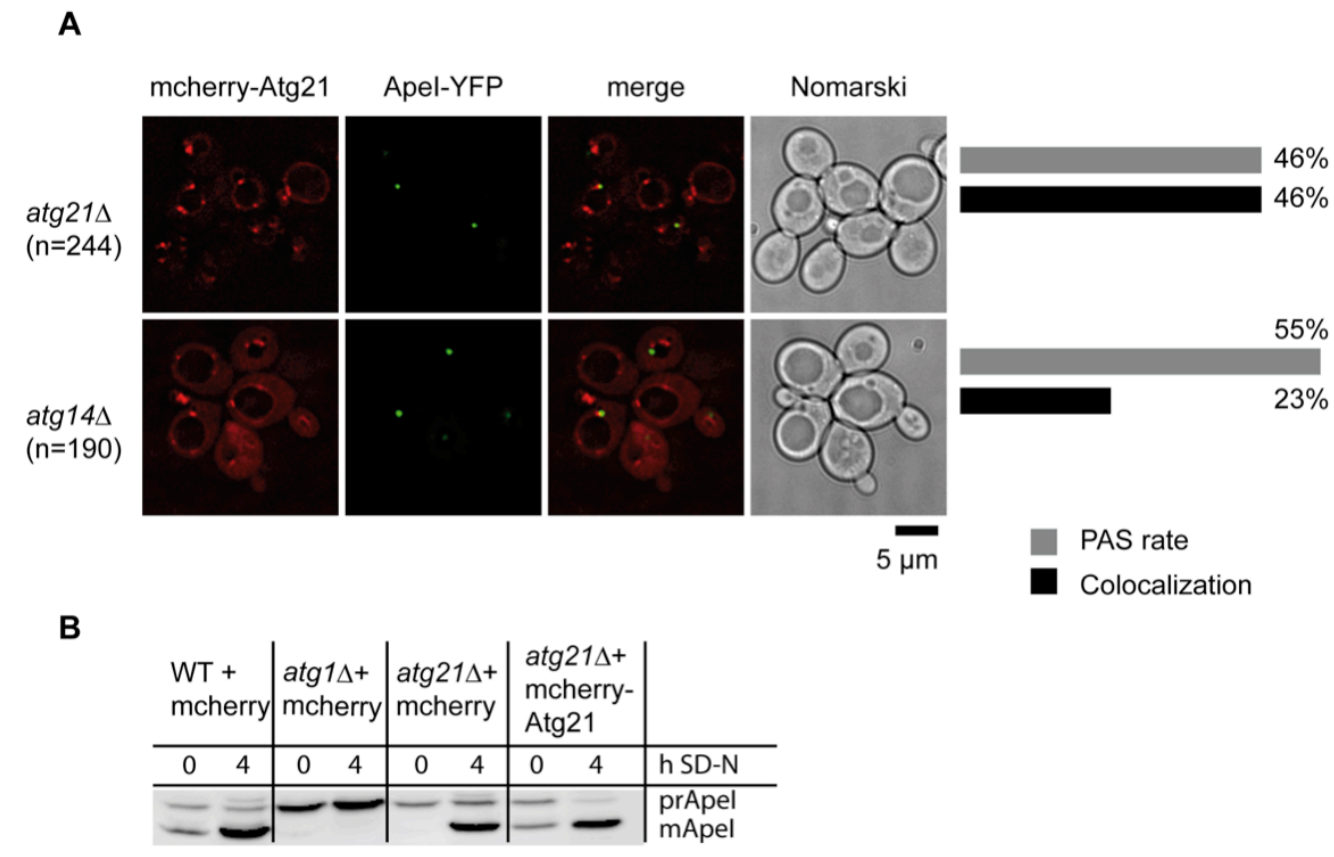

Figure 13: Complementation study of mCherry-Atg21 by monitoring ApeI maturation and determination of its PAS rate.

(A) Growing atg21 $\Delta$ and $\operatorname{atg} 14 \Delta$ cells expressing mCherry-Atg21 and ApeI-YFP in selective medium containing $0.3 \mathrm{mM}$ methionine were visualized by direct fluorescence microscopy (DeltaVision Deconvolution microscope). Pictures were deconvoluted using SoftWoRX (Applied Precision) software. PAS rate and Colocalization rate were determined as described before (Figure 11). The number of counted cells (n) is indicated respectively. (B) Indicated strains expressing mCherry-Atg21 or mCherry alone were grown in selective medium containing $0.3 \mathrm{mM}$ methionine. Mid-log cells $(0 \mathrm{~h})$ or cells starved for $4 \mathrm{~h}$ in SD-N $(4 \mathrm{~h})$ were alkaline lysed and immunoblotted. Using an anti-Apel antibody, precursor ApeI (prApeI) and matured ApeI (mApeI) were detected.

To further prove the functionality of the mCherry-Atg21 construct, its ability to rescue the ApeI maturation defect of atg21 cells was tested (Figure $13 \mathrm{~B}$ ). ApeI is expressed as a preform (prApeI) in the cytosol, where it forms large 
dodecamers. These complexes are transported under growing conditions via the selective Cvt pathway, or under starvation via macroautophagy to the vacuole, where precursor ApeI is matured (mApeI). For the ApeI maturation analysis, WT, $\operatorname{atg} 1 \Delta$ and $\operatorname{atg} 21 \Delta$ cells expressing the empty vector and atg21 cells expressing mCherry-Atg21 were grown to mid-log phase ( $0 \mathrm{~h}$ SD-N) and subsequently starved for four hours in SD-N (4 h SD-N). Samples were taken at the indicated time points, alkaline lysed and subjected to immunoblotting. prApeI and mApeI were detected using an anti-ApeI antibody (Figure $13 \mathrm{~B}$ ). In growing WT cells, about half of ApeI was matured, whereas after $4 \mathrm{~h}$ of nitrogen starvation most of ApeI was found in its matured form. Atg1 is a core autophagy protein, therefore the maturation of ApeI was blocked under both growing and starvation conditions in atg1د cells. Since Atg21 is required under growing conditions, ApeI maturation was completely blocked under those conditions and severely impaired in starved atg21 cells. mCherry-Atg21 was able to fully rescue the ApeI maturation defect of atg21 cells under both, growing and starvation conditions, implicating that the mCherry-Atg21 construct was functional (Figure 13 B).

\subsubsection{Atg21 is not transported into the vacuole}

Within the autophagic process, the outer membrane of the completed, doublemembrane layered autophagosome fuses with the vacuolar membrane to release the still one membrane layered autophagic body into the vacuole. In the vacuole the autophagic body is lysed and its contents are degraded. From the autophagy related proteins only those on the inside of the autophagosome are transported into the vacuole. Atg8 is evenly distributed across the outer and inner membrane of the autophagosome. The part, located on the inner membrane, is trapped inside the autophagosome and transported into the vacuole, whereas the Atg12Atg5/Atg16 complex is only associated to the outer membrane and therefore not transported into the vacuole (Kirisako et al., 2000; Mizushima et al., 2001; Suzuki et al., 2001). Part of Atg21 was successfully detected at the PAS (see chapter 4.2.1). The PAS localization was dependent on Atg14 and therefore on the presence of PI3P on the forming autophagosome, suggesting that Atg21 is at least peripherally membrane associated there. To analyze, if Atg21 is evenly 
distributed on the inner and outer membrane of the forming autophagosome or if it is only associated to the outer membrane, the transport of Atg21 into the

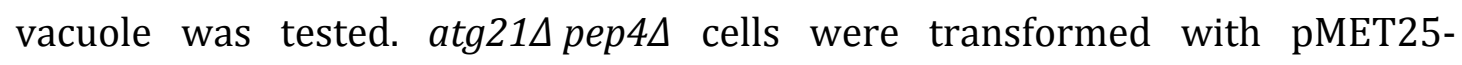
mCherry-Atg21 and pRS313-GFP-Atg8, grown to an $\mathrm{OD}_{600}$ of 2 to 3 and analyzed by direct fluorescence microscopy (Figure 14). Again, $0.3 \mathrm{mM}$ methionine was added to the media to induce endogenous mCherry-Atg21 expression level.

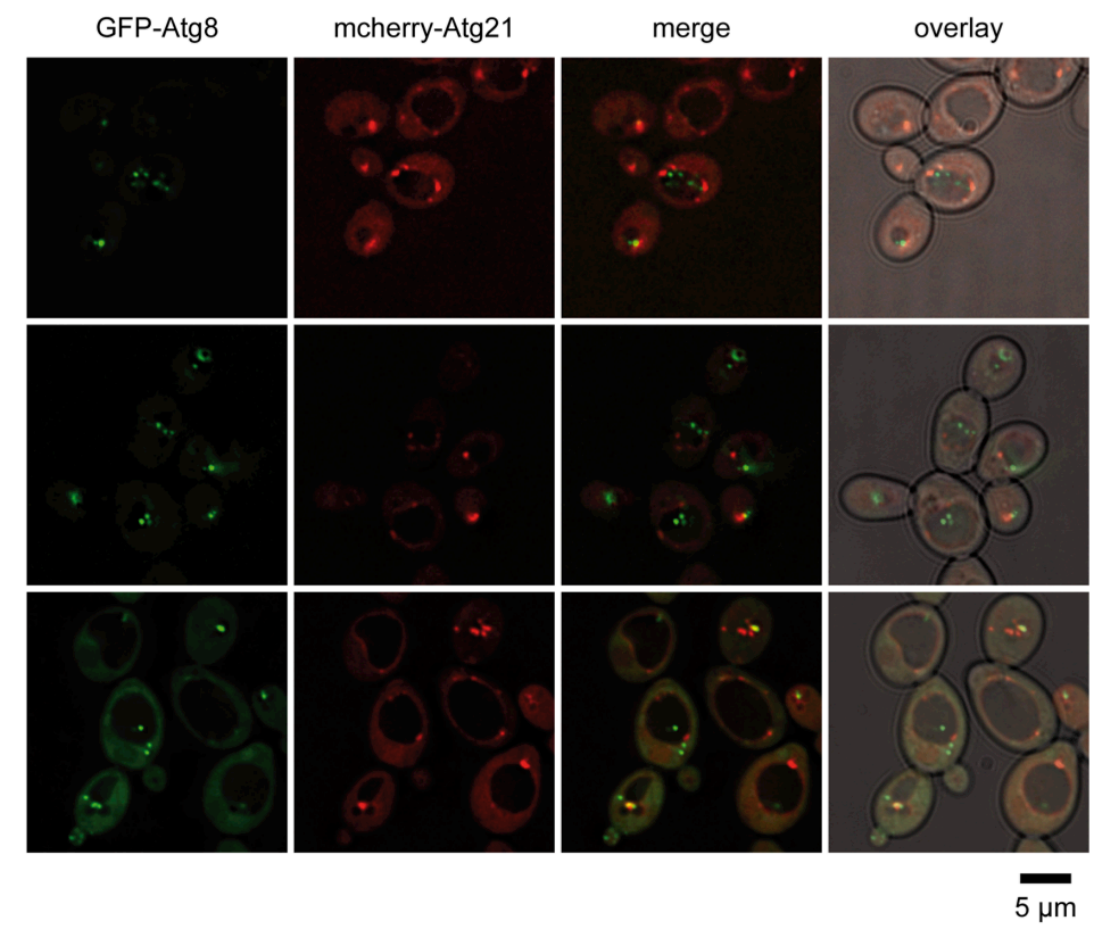

Figure 14: mCherry-Atg21 is not transported into the vacuole

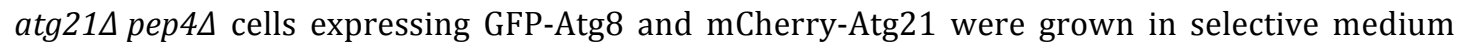
containing $0.3 \mathrm{mM}$ methionine to an $\mathrm{OD}_{600}$ of 2 to 3 . Pictures were taken using a DeltaVision fluorescence microscope equipped with a mCherry and GFP filter set and deconvoluted using SoftWoRX (Applied Precision) software.

In cells lacking the vacuolar aspartyl protease (Pep4), the autophagic bodies are not lysed within the vacuole and accumulate over the time. In this approach, they were visible as green dots within the vacuole by labeling with GFP-tagged Atg8. But none of those dots showed an additional red fluorescence for mCherryAtg21. Furthermore, no red fluorescent dots were visible within the vacuole at all. Taken together, mCherry-Atg21 is most probably not transported into the vacuole and therefore not evenly distributed across the autophagosomal membranes. 


\subsection{Identification of interaction partner of Atg21}

Atg21 is a WD40 repeat $\beta$-propeller. WD-repeat propeller proteins commonly create a stable platform for coordinating and mediating assembly of protein complexes (Xu and Min, 2011). Therefore, they often interact reversibly with several protein interaction partners. Identification of potential interaction partner of Atg21 will elucidate its molecular function and its role in the autophagic process.

\subsubsection{Atg21 is part of a high molecular weight complex}

\subsubsection{Gelfiltration analysis of Atg21}

The PAS localization of the Atg21 homologue Atg18 is specifically mediated by interaction with both, PI3P and Atg2 (Obara et al., 2008b; Rieter et al., 2013) . Obara et al. (2008) detected a large Atg18-Atg2 complex in gelfiltration (GF). Intriguingly, this large complex disappeared in the absence of Atg2. The gelfiltration approach was used to clarify, if Atg21 can also be found in a high molecular weight complex. Finding suitable conditions for purification of the Atg21 complex might lead to the identification of potential Atg21 interaction partner. For this purpose, pRS316-Atg21-TAP was transformed into atg21 pep $4 \Delta$ cells. First, the cells were resuspended in Obara's GF-buffer (20 mM Tris/HCl pH 8.0, 150 mM KCL, 5 mM MgCl 2,1 mM PMSF, 1\% Triton X100 , protease inhibitors) and pneumatically lysed with a pressure of about 1500 bar in three cycles using the homogenizer EmulsiFlex C3 (Avestin). The cell lysate was cleared by ultracentrifugation at $100000 \mathrm{xg}$ for $30 \mathrm{~min}$ and subjected to size exclusion chromatography on a Superose 6 column. The cell lysate was eluted from the column at a flow rate of $0.5 \mathrm{ml} / \mathrm{ml}$ and $0.8 \mathrm{ml}$ fractions were collected as it was described by Obara et al. (2008). The fractions were precipitated, dissolved in Laemmli buffer and processed for immunoblots (Figure 15). For molecular weight determination the immunoblot was decorated with antibodies against commonly used proteins with known size as ApeI and the phosphoglycerate kinase (PGK). ApeI is known to form dodecamers with a predicted molecular weight of about $670 \mathrm{kDa}$ (Morales Quinones et al., 2012) and was present in fractions 16 to 17 . PGK is a housekeeping protein and is, 
independent from most conditions, randomly distributed in the cytosol. PGK has a molecular weight of about $45 \mathrm{kDa}$ and was eluted in fractions 22 to 24 .

A

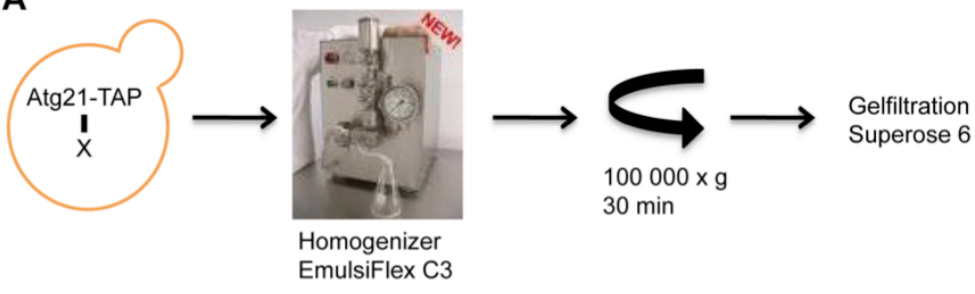

B

$\operatorname{atg} 21 \Delta$ pep $4 \Delta+$ Atg21-TAP

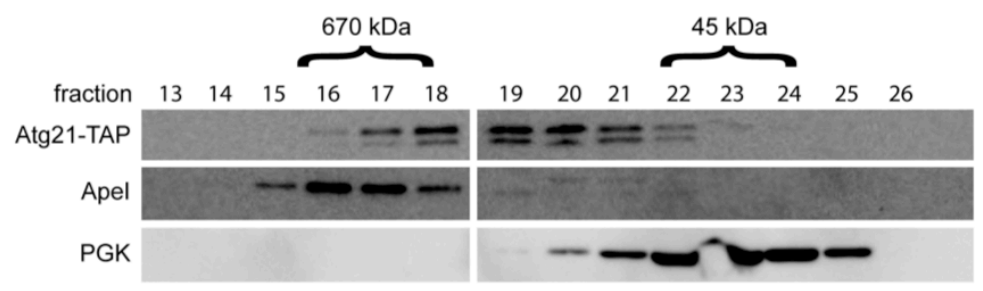

C

$\operatorname{atg} 21 \Delta$ pep $4 \Delta+$ Atg21-TAP

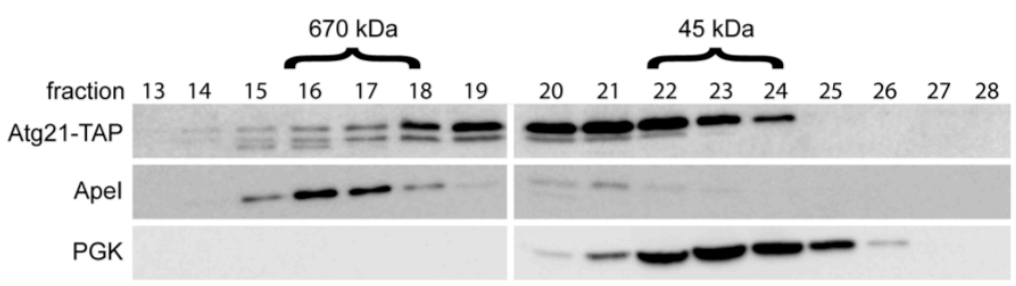

D

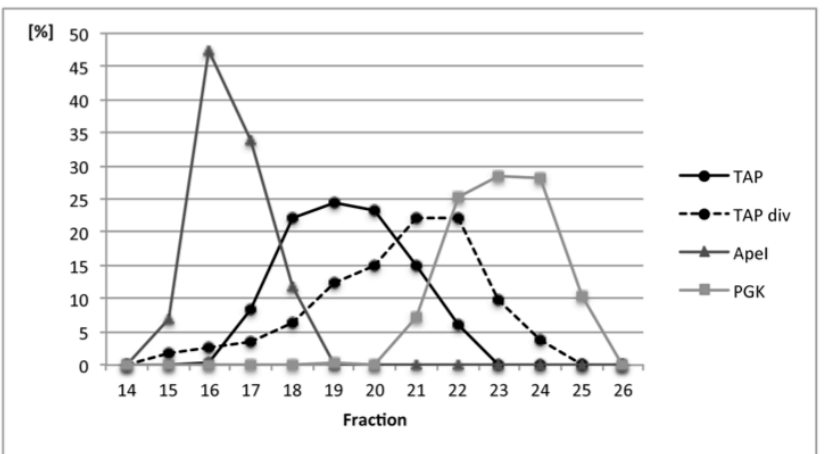

Figure 15: Atg21-TAP was present in a sensitive high molecular weight complex in gelfiltration

(A) Scheme of cell lysis and sample preparation for gelfiltration using the Homogenizer EmulsiFlex C3 (Avestin) (picture adapted from: http://www.avestin.com/ German/c3page.html). (B) atg21 pep $4 \Delta$ cells expressing Atg21-TAP were treated as shown in (A) and subjected to size exclusion chromatography on a Superose 6 column. The cell lysate was eluted from the column at a flow rate of $0.5 \mathrm{ml} / \mathrm{ml}$ and $0.8 \mathrm{ml}$ fractions were collected as it was described in Obara et al. (2008). Fractions were precipitated and analyzed by immunoblotting using anti-TAP, -ApeI and -PGK antibodies. (C) Part of Atg21-TAP is also present in its monomeric form. (D) Quantification of the distribution of the individual proteins in the respective fractions using AIDA software. 
Atg21-TAP, with a predicted molecular weight of about $76 \mathrm{kDa}$, was eluted in fractions 18 to 20 . Since fraction 18 corresponded to a molecular weight of about $670 \mathrm{kDa}$, a part of Atg21-TAP was present in a large complex (Figure $15 \mathrm{~B}+\mathrm{D}$ ). Unfortunately, these data were not reproducible. In further experiments, the main part of Atg21-TAP was present in fractions 21 to 22. These fractions corresponded to the predicted molecular weight of Atg21-TAP and are therefore likely to represent its monomeric form (Figure $15 \mathrm{C}+\mathrm{D}$ ). To keep the apparent sensible large Atg21 complex intact, the cells were lysed in a very mild and preservative way. The cells were first converted to spheroblasts, solubilized in lysis buffer (0.8 M sorbitol, $10 \mathrm{mM}$ MOPS pH 7.2, 1 mM EDTA, 2 mM PMSF, protease inhibitors) and then mechanically lysed with 30 beats of a cell homogenizer. The cell lysate was cleared and subjected to a Superose 6 column as described above (Figure $16 \mathrm{~A}$ ). Again the immunoblot was decorated with antibodies against marker proteins as the ApeI complex $(670 \mathrm{kDa})$ and the carboxypeptidase Y (CPY; $65 \mathrm{kDa}$ ). Under these conditions, the ApeI complex eluted in fractions 12 to 13, whereas CPY was present in fractions 16 to 17. Atg21-TAP was almost completely eluted in fractions 9 to 11, which corresponded to a molecular weight of $670 \mathrm{kDa}$ and more. So Atg21-TAP seemed to be present in a high molecular weight complex (Figure $16 \mathrm{~B}$ ). If the same cell lysis procedure was carried out by adding 0.5\% Triton X-100 to the lysate after cell homogenization, a small amount of Atg21-TAP was present in additional fractions corresponding to lower molecular weights (Figure 16 C). 
A

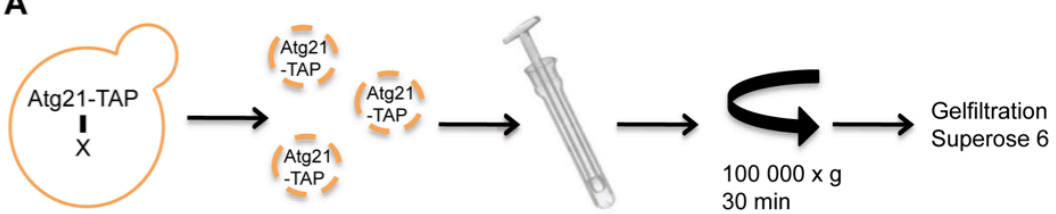

B

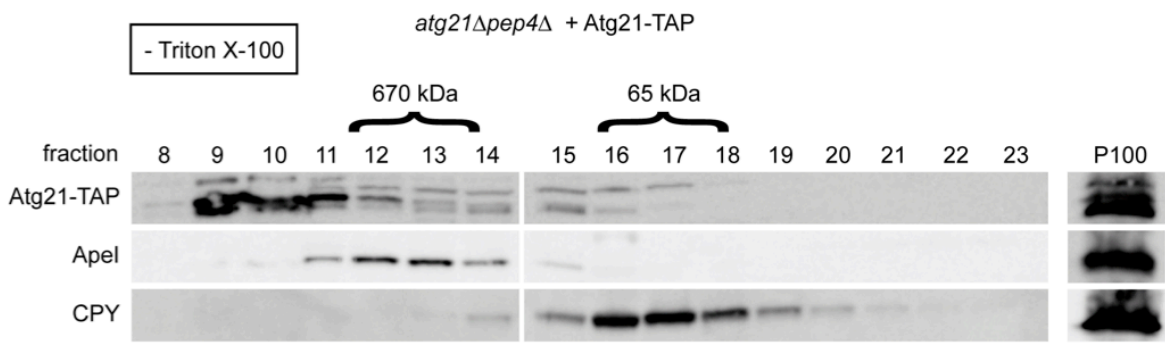

C $+0.5 \%$ Triton $\mathrm{X}-100 \quad \operatorname{atg} 21 \Delta$ pep $4 \Delta+$ Atg21-TAP

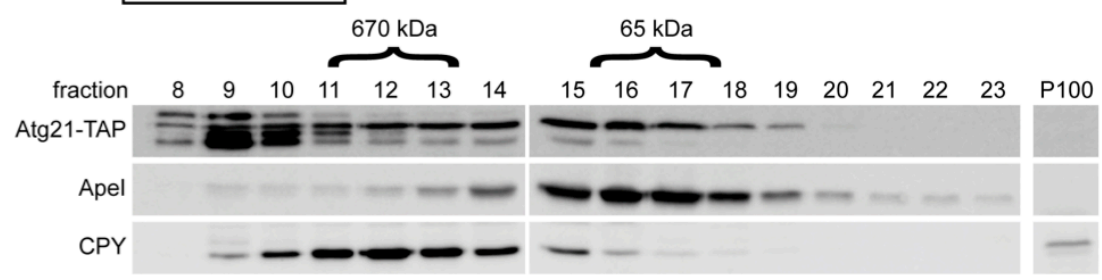

D
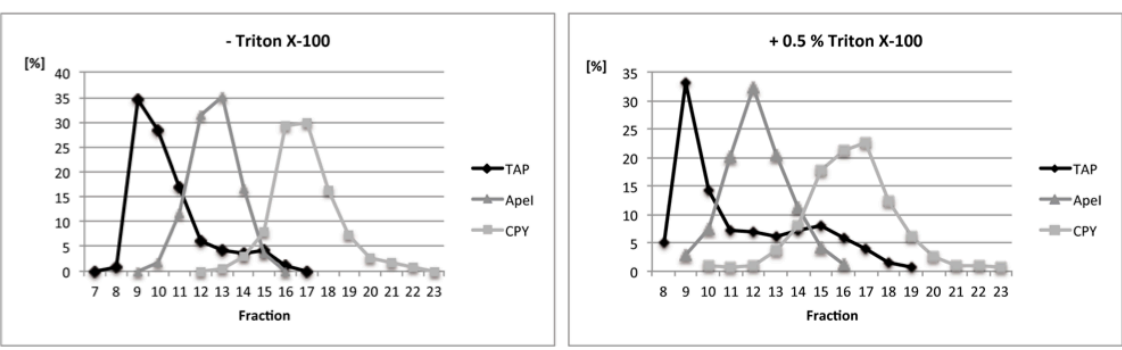

Figure 16: Alternative mild cell lysis procedure keeps the sensitive high molecular weight Atg21 complex intact

(A) Scheme of mild cell lysis procedure and sample preparation for gelfiltration using a

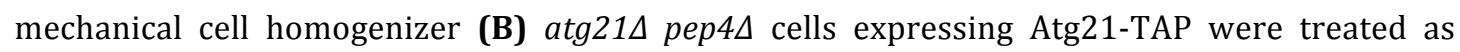
shown in (A) without detergence and analyzed by gelfiltration as described above. Distribution pattern of the respective proteins were analyzed by immunoblotting using anti-TAP, anti-ApeI

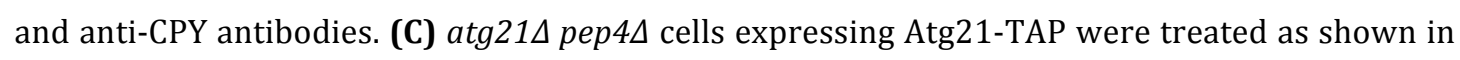
(A) with addition of Triton X-100 to the cell lysate after cell homogenization. Cell lysate was analyzed by gelfiltration as described in (B). (D) Quantification of the distribution of the individual proteins in the respective fractions using AIDA software.

In conclusion, a mild cell lysis procedure was established that keeps the sensitive high molecular weight Atg21 complex intact. Therefore, this cell lysis procedure 
was used in the following experiments to further analyze the high molecular weight complex and to identify potential interaction partners of Atg21.

\subsubsection{Sorbitol density gradient centrifugation}

Density gradient centrifugation was used to further analyze the Atg21 high molecular weight complex. In this approach, proteins and protein complexes can be separated by their mass but also density and shape. In principle, a density gradient is created in a centrifugation tube. The cell lysate is placed on the top. Applied to a strong centrifugal field, proteins migrate along the gradient until they reach the point that corresponds to their density.

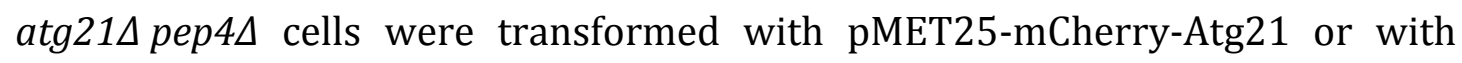
pMET25-mCherry as a negative control. Cells were harvested in growing phase and mildly lysed in the presence of Triton X-100 as described before (chapter 4.3.1.1). The cell lysate was placed on the top of a $20 \%$ to $50 \%$ glycerol density gradient and subsequently centrifuged for four hours at $259000 \mathrm{xg}$ (Figure $17 \mathrm{~A}$ ). Afterwards ten fractions were collected beginning from the top of the gradient and precipitated with TCA. The pellets were dissolved in Laemmli buffer and processed for immunoblots (Figure $17 \mathrm{~B}$ ).

A

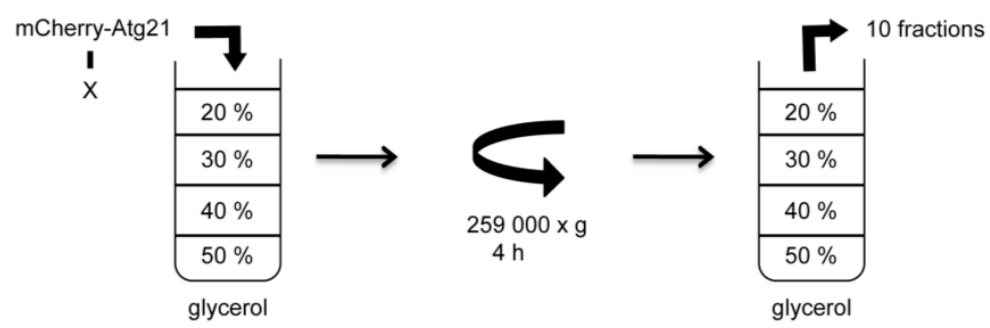

B

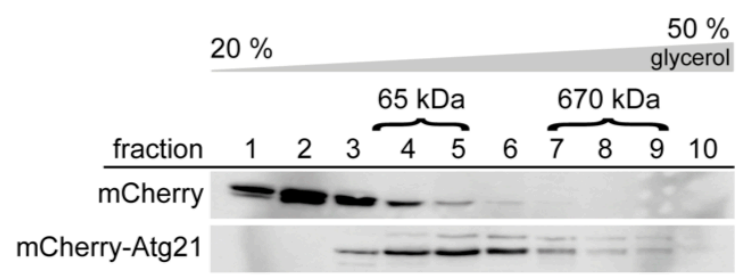

Figure 17: The high molecular weight Atg21 complex could not be detected after glycerol density gradient centrifugation

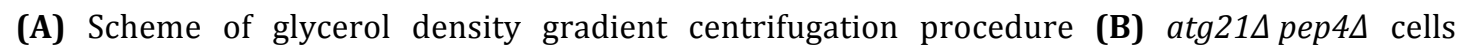
expressing mCherry-Atg21 or mCherry alone were grown in selective medium in the absence of methionine to induce protein expression, converted to spheroblasts and mechanically lysed 
using a cell homogenizer. Cell lysate was subjected to a $20 \%$ to $50 \%$ glycerol density gradient centrifugation as described in (A). Ten fractions were collected beginning from the top of the gradient and analyzed by immunoblot using an anti-ApeI, -CPY and -Red (specifically recognizes mCherry) antibody.

As a molecular weight marker monomeric CPY (65 kDa) and the ApeI complex $(670 \mathrm{kDa})$ were used (data not shown). CPY was found in fractions 4 to 5 , the ApeI complex in fractions 7 to 8 . As expected, mCherry with a molecular weight of about $30 \mathrm{kDa}$ was present in the first fractions corresponding to low density (fractions 1 to 3). mCherry-Atg21 (82.5 kDa) was detected in fraction 4 to 6 , corresponding to the fractions where CPY was present. So mCherry-Atg21 was rather present in its monomeric form than in a complex. As seen in the gelfiltration analysis, the Atg21 high molecular weight complex was extremely sensitive (chapter 4.3.1.1). One possibility for the instability of the complex could be intolerance for glycerol. Sorbitol was present in the cell lysis buffer, where the Atg21 high molecular weight complex was stable as seen in gelfiltration (chapter 4.3.1.1). Therefore, the glycerol in the gradient was exchanged with sorbitol solutions with the same density, respectively (Figure $18 \mathrm{~A}$ ). Cell lysate of atg21 pep $4 \Delta$ cells expressing pMET25-mCherry-Atg21 or pMET25-mCherry as a control were loaded on a $0.9 \mathrm{M}$ to $2.5 \mathrm{M}$ sorbitol density gradient and centrifuged for four hours at $259000 \mathrm{xg}$ (Figure 18 A). Again, ten fractions beginning from the top of the gradient were taken and analyzed using anti-Red, anti-ApeI and anti-CPY antibodies in immunoblotting (Figure $18 \mathrm{~B}$ ). The ApeI complex (670 kDa) was detected in fractions 7 to 9, whereas CPY (67 kDa) was mainly present in fractions 3 to 4 . As expected, mCherry was again present in the first fractions (fractions 2 to 3 ) corresponding to low density and molecular weight. In contrast, mCherry-Atg21 was found in fractions 4 to 8 and was therefore at least partly present in fractions corresponding to high molecular weight and density. 
A

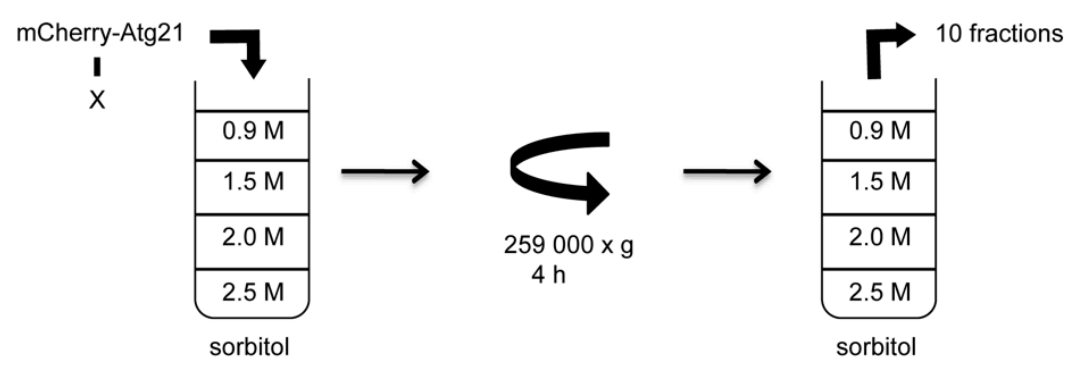

B
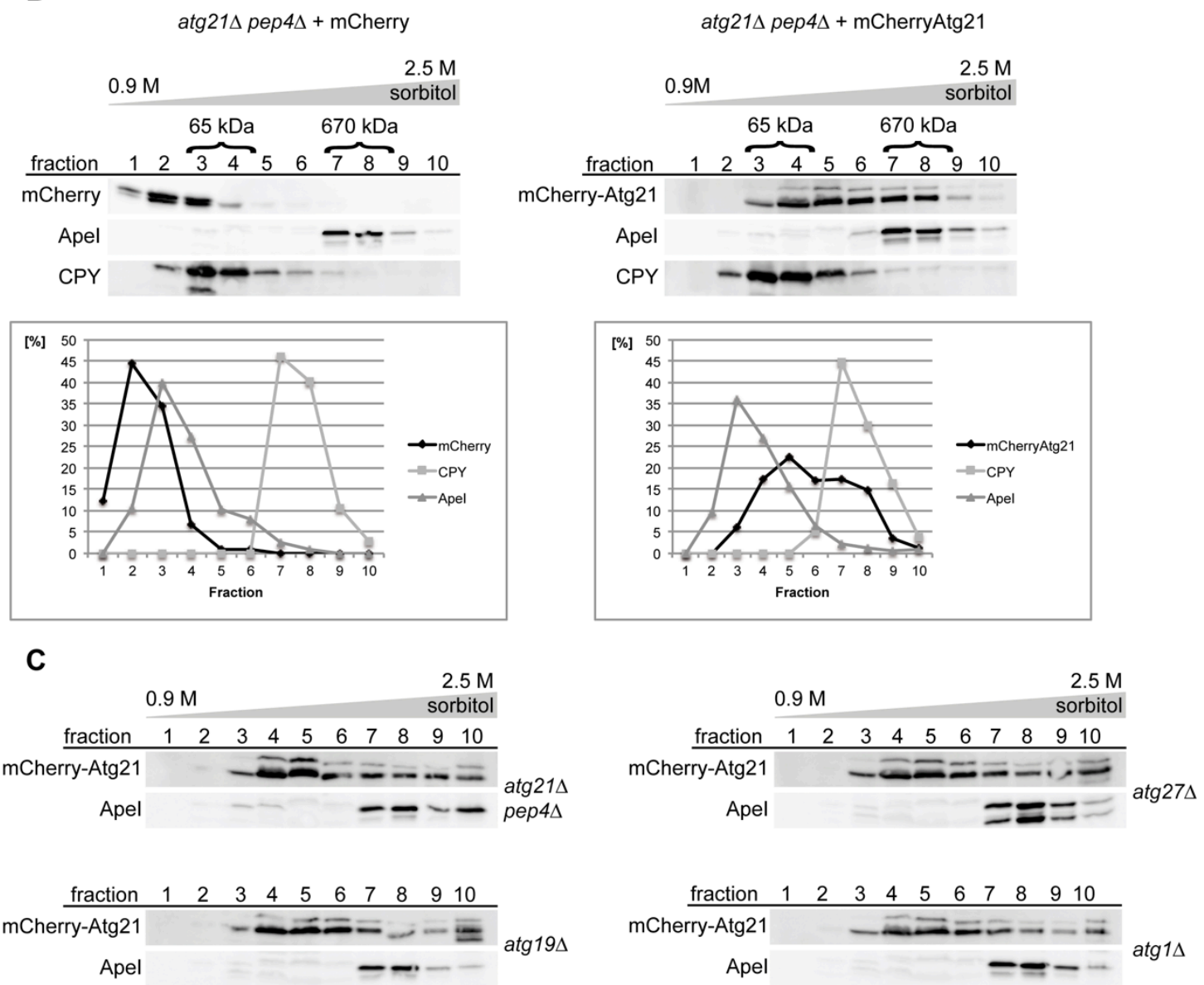

Figure 18: mCherry-Atg21 is present as a high molecular weight complex in sorbitol density gradient centrifugation

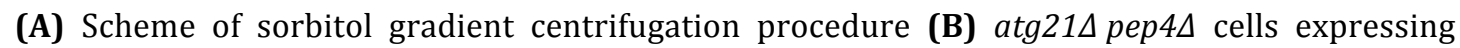
mCherry-Atg21 or mCherry were grown in selective medium without methionine to induce protein expression. Cell lysates were prepared with $0.5 \%$ Triton X-100 as described before and subjected to sorbitol density gradient centrifugation as described in (A). Ten fractions beginning from the top of the gradient were taken, TCA precipitated and analyzed by immunoblot using anti-Red, anti-ApeI and anti-CPY antibody. The distribution pattern of the respective proteins

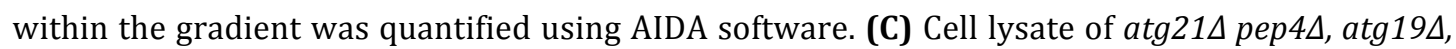
$\operatorname{atg} 27 \Delta$ and $\operatorname{atg} 1 \Delta$ cells expressing mCherry-Atg21 were prepared in the absence of detergence as described before and subjected to sorbitol gradient centrifugation as described in (A) and (B). 
Gelfiltration revealed that detergence influenced the stability of the Atg21 high molecular weight complex (chapter 4.3.1.1). To determine, if this could also be true for sorbitol density gradient centrifugation, cells expressing pMET25mCherry-Atg21 were lysed in the absence of detergence and cell lysate were again loaded on the top of a $0.9 \mathrm{M}$ to $2.5 \mathrm{M}$ sorbitol gradient. Indeed, a part of mCherry-Atg21 could be detected in fractions corresponding to high molecular weight and density (fraction 9 and 10;Figure $18 \mathrm{C}$ ).

Sorbitol gradient centrifugation was performed with different knock out strains expressing pUG36-mCherry-Atg21 to determine if any of these proteins had an influence on the formation of the Atg21 high molecular weight complex or might even be a component of it. To preserve the complex, cell lysates were prepared in the absence of detergence. Surprisingly, nor Atg19, Atg27 or Atg1 changed the distribution of mCherry-Atg21 in the sorbitol gradient compared to atg $21 \Delta$ pep $4 \Delta$ cells (Figure $18 \mathrm{C}$ ). So none of the tested proteins was involved in the formation of the high molecular weight Atg21 complex or was a stoichiometric component of it.

\subsubsection{Pull down und mass spectrometry analysis to identify potential Atg21 interaction partner}

Pull down experiments were used as one method to identify potential Atg21 interaction partner. Therefore, recombinant 6xHis-SUMO-Atg21 was incubated with crude yeast cell extract and the co-precipitated proteins were identified by mass spectrometry analysis. Atg21 was N-terminally fused to SUMO and an additional six histidine tag for purification (6xHis-SUMO-Atg21) (Figure $19 \mathrm{~A}$ ). To exclude those proteins, which bound to SUMO, SUMO alone, N-terminally tagged with six histidines, was used as a negative control (6xHis-SUMO). Recombinant 6xHis-SUMO-Atg21 and 6xHis-SUMO was purified via Ni-NTAbeads (see chapter 3.6.11). Samples were taken after E. coli cell lysis (E. coli L) and after cell lysate clearance by centrifugation (E.coli S) to prove efficient expression and the solubility of 6xHis-SUMO-Atg21. 6xHis-SUMO-Atg21 and 6xHis-SUMO coupled to Ni-NTA beads were subsequently incubated with crude cell extract of $\operatorname{atg} 21 \Delta$ cells expressing mCherry-Atg19 and GFP-Atg8 (yeast L). Afterwards the supernatant containing the unbound proteins (yeast S) was 
removed. After extended washing, 6xHis-SUMO, 6xHis-SUMO-Atg21 and all associated proteins (E) were eluted from the beads by adding NuPAGE ${ }^{\circledR}$ sample buffer (Life Technologies) and boiling at $72^{\circ} \mathrm{C}$ for $10 \mathrm{~min}$. The samples were also loaded on a SDS-PAGE and processed for immunoblotting. (Figure 19 B).

A

\section{6xHIS SUMO Atg21}

B

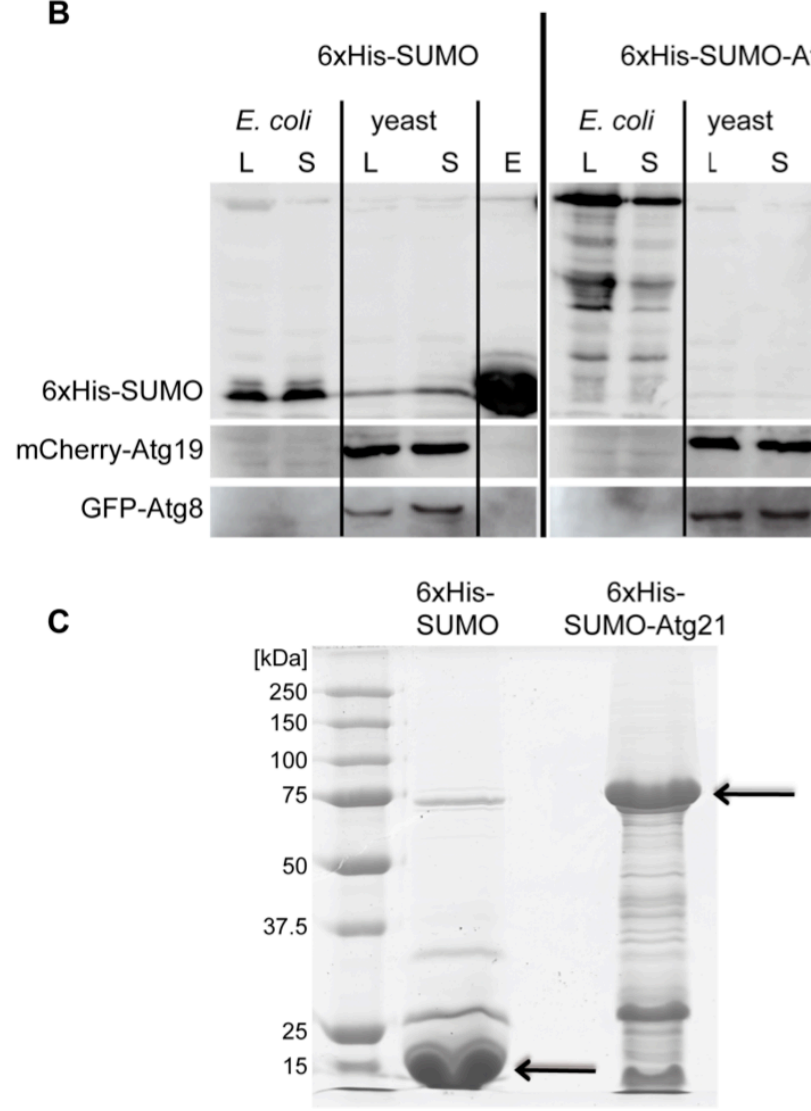

D

\begin{tabular}{c|c|c|c|c|c|c|} 
& \multicolumn{3}{|c|}{ 6xHis-SUMO } & \multicolumn{3}{c|}{ 6xHis-SUMO-Atg21 } \\
$\begin{array}{c}\text { identified } \\
\text { proteins }\end{array}$ & $\begin{array}{l}\text { peptide } \\
\text { number }\end{array}$ & $\begin{array}{l}\text { peptide } \\
\text { coverage [\%] }\end{array}$ & $\begin{array}{l}\text { quantitative } \\
\text { values }\end{array}$ & $\begin{array}{l}\text { peptide } \\
\text { number }\end{array}$ & $\begin{array}{l}\text { peptide } \\
\text { coverage [\%] }\end{array}$ & $\begin{array}{l}\text { quantitative } \\
\text { values }\end{array}$ \\
\hline Tor1 & 0 & 0 & 0 & 78 & 40 & 65 \\
\hline Tor2 & 0 & 0 & 0 & 51 & 29 & 43 \\
\hline Tc089 & 2 & 2.4 & 3 & 27 & 45 & 20 \\
\hline Kog1 & 0 & 0 & 0 & 42 & 33 & 30 \\
\hline Lst8 & 1 & 2.3 & 6 & 4 & 19 & 4 \\
\hline
\end{tabular}

Probability Legend: $\square$ over $95 \% \quad \square 50 \%$ to $79 \% \quad \square 0 \%$ to $19 \%$

Figure 19: Pull down analysis of SUMO-Atg21

(A) Scheme of the 6xHis-SUMO-Atg21 construct (B) Recombinant 6xHis-SUMO and 6xHis-SUMOAtg21 were purified using Ni-NTA beads and incubated with crude extract of growing atg21 
cells expressing mCherry-Atg19 and GFP-Atg8. The respective yeast and E. coli lysates (L), cleared lysate (E. coli S), unbound fraction (yeast $S$ ) and the purified proteins (E) were subjected to SDS-PAGE analyzed by immunoblotting using anti-His, anti-Red and anti-GFP antibodies. (C) Isolated proteins (E) were further analyzed by Coomassie staining and subjected to mass spectrometry (MS) analysis for identification. (D) Identified members of the TORC1 complex are listed. The respective peptide number, coverage and quantitative values determined by the Scaffold3 software are shown.

6xHis-SUMO-Atg21 was present in both the E. coli lysate and supernatant after centrifugation in comparable amounts, suggesting that recombinant 6xHisSUMO-Atg21 was soluble. Furthermore, 6xHis-SUMO-Atg21 could be successfully purified in sufficient amounts (see E in Figure 19 B and C). Though the potential Atg21 interaction partner GFP-Atg8 did not co-precipitate with 6xHis-SUMOAtg21 (see below), the Cvt receptor mCherry-Atg19 could be specifically coprecipitated. Since Atg21 is required for the Cvt pathway, this indicates that 6xHis-SUMO-Atg21 was functional.

In addition, the eluates of the 6xHis-SUMO-Atg21 and 6xHis-SUMO pull down were loaded on a SDS-PAGE and analyzed by colloidal Coomassie staining. Only three additional bands to 6xHis-SUMO were visible in the 6xHis-SUMO eluate, whereas several additional bands representing potential interaction partner were prominent in the eluate of the 6xHis-SUMO-Atg21 pull down. For identification of the potential interaction partner, the eluate samples were subjected to mass spectrometry analysis (performed by Bioanalytical Mass Spectrometry Group of Prof. Henning Urlaub, Universität Göttingen). In total, 912 proteins were identified. Based on quantitative values, those proteins were excluded that were present in the negative control (6xHis-SUMO) in an equivalent or higher amount compared to the 6xHis-SUMO-Atg21 sample. Regardless of the low quantitative values of some of the proteins, 728 proteins were specifically identified that bind to 6xHis-SUMO-Atg21. Curiously, none of them represented an Atg protein. However, all components of the target of rapamycin complex 1 (TORC1) were identified (Figure 19 D). Among other functions, TORC1 is a key actor in autophagy regulation. TORC1 consists of Tco89, Kog1, Lst8 and either Tor1 or Tor2 (Loewith and Hall, 2011). Tor1, Tor2, Tco89 and Kog1 were present in high quantitative values in the 6xHis-SUMO- 
Atg21 sample. The negative control 6xHis-SUMO showed no binding to the subunits of TORC1 other than Lst8. Though the quantitative value was slightly higher in the negative control, only one peptide was detected leading to a peptide coverage of only $2.3 \%$. In contrast, in the 6xHis-SUMO-Atg21 pull down four peptides of Lst8 were identified leading to a peptide coverage of 19\%. Based on this, the probability in the 6xHis-SUMO pull down was limited to only $50 \%$ compared to $100 \%$ in the 6xHis-SUMO-Atg21 sample (Figure $19 \mathrm{D}$ ). However, these data supported an interaction of 6xHis-SUMO-Atg21 with TORC1.

\subsubsection{Split-ubiquitin analysis of Atg21}

The split-ubiquitin assay represents an analytic tool for measurement of protein interaction in living yeast cells (Müller and Johnsson, 2008) and was used for a large-scale search for potential interaction partners of Atg21.

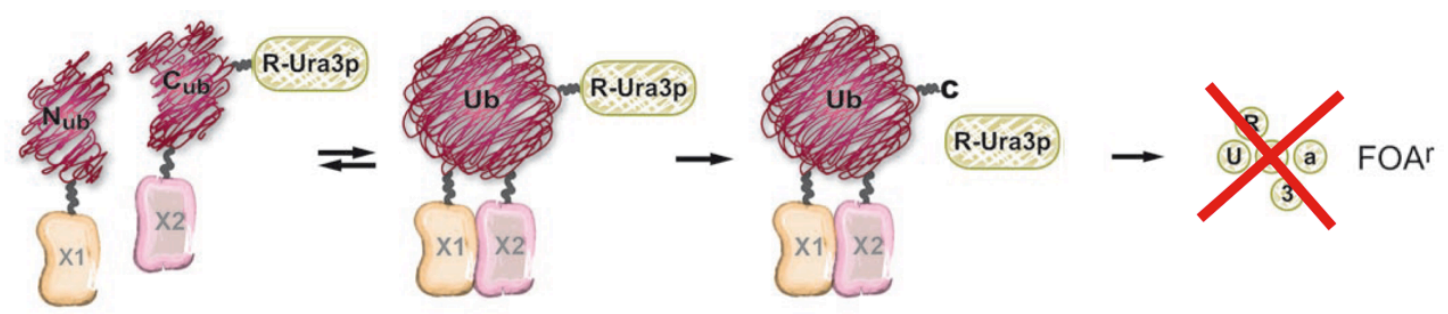

Figure 20: Scheme of the principle of the split-ubiquitin assay (adapted from (Müller and Johnsson, 2008))

For this purpose, ubiquitin is split into two halves and fused to two proteins of interests (Figure 20). In detail, the N-terminal half of ubiquitin (Nui) was fused to the N-terminus of one of the proteins (prey) and the C-terminal half of ubiquitin (Cub) was fused to the C-terminus of the other protein (bait). In addition, the Cub fragment was attached to the reporter protein R-Ura3. Ura3 represents the orotidine 5-phosphate decarboxylase and plays a crucial role in the synthesis of uracil. Furthermore, it is able to convert 5-Fluoroorotic acid (5-FOA) into the toxic compound 5-fluorouracil, so that it can be used as both a positive and negative reporter protein. If both proteins of interest interact with each other, the two halves of ubiquitin reassemble into native-like ubiquitin. Ubiquitin can now be recognized by ubiquitin specific proteases, which in turn cleave off the reporter protein R-Ura3. Because an arginine residue was introduced at the CubUra3 junction, the free R-Ura3 protein exposes an N-terminally arginine residue, 
which is according to the $\mathrm{N}$-end-rule a destabilizing amino acid and leads to the rapid degradation of R-Ura3 and therefore to uracil auxotrophy (Müller and Johnsson, 2008). Consequently, cells expressing interacting proteins are able to grow on plates containing 5-FOA but not on plates lacking uracil. The other way round, if the two proteins do not interact with each other, the two halves of ubiquitin do not reassemble to native ubiquitin. Therefore, the reporter protein R-Ura3 is not cleaved off and degraded. Thus, cells expressing non-interacting proteins are able to grow on plates lacking uracil but not on plates containing 5 FOA.

Atg21 was tested for an interaction with several autophagy related proteins. $1 \mathrm{OD}_{600}$ cells expressing Atg21-Cub and the respective Nui-Atg protein were diluted in 10-fold steps and spotted on CM-Trp-His (growth control), on CM-TrpHis +FOA (growth indicated interaction of the proteins) and on CM-Trp-His-Ura (growth indicated no interaction of the proteins). The expression of the proteins of interest was induced by the addition of either $100 \mu \mathrm{M} \mathrm{CuSO}_{4}$ (Nui) or $250 \mu \mathrm{M}$ methionine ( $\mathrm{Cub}$ ) to the selective medium (Laser et al., 2000). After two to three days the growth pattern was analyzed. Cells expressing the interacting proteins Ste14-Cub and Nui-Ubc6 served as a positive control, cells expressing Ste14-Cub and the empty vector (pRS314) as a negative control (Krick et al., 2010). Atg21Cub was tested for an interaction with Nui-Atg1, -Atg5, -Atg7, -Atg8, -Atg9, Atg12, -Atg16, -Atg18, -Atg19, -Atg21, -Atg23, -Atg27, -Hsv2, -Trs85 and -Vam1. Three of them, Atg27, Atg8 and Atg16 showed a potential interaction and have been analyzed in more detail.

\subsubsection{Interaction studies of Atg21 und At27}

$\operatorname{Atg} 27$ is a type I membrane protein. It directly interacts with the second autophagy related membrane protein Atg9, which in turn interacts with the cytosolic protein Atg23 to form a trimeric complex. All three proteins cycle between the PAS and a peripheral pool and are dependent on each other for efficient cycling (Legakis et al., 2007; Yen et al., 2007). Comparable to Atg21, Atg27 is absolutely required for the Cvt pathway, whereas macroautophagy is only significantly reduced in atg27 cells (Yen et al., 2007). Both, PI3P binding and interaction with its interacting partner Atg2, are crucial for proper PAS 
localization of the Atg21 homologue Atg18 (Rieter et al., 2013). As a membrane protein required for the Cvt pathway, Atg27 would be a good candidate for the missing interaction partner of Atg21 at the PAS.

\subsubsection{Atg27 and Atg21 interact with each other in split-ubiquitin assay}

In the split-ubiquitin assay, Atg21-Cub showed neither an interaction with NuiAtg9 nor Nui-Atg27 (Figure 21 A). Furthermore, there was no interaction detectable between Atg23-Cub and Nui-Atg21 or its indirect binding partner Nui-Atg27 (Figure $21 \mathrm{~B}$ ). In contrast, an interaction of Atg23-Cub and its direct interaction partner Nui-Atg9 was detected, serving as a positive control.

A

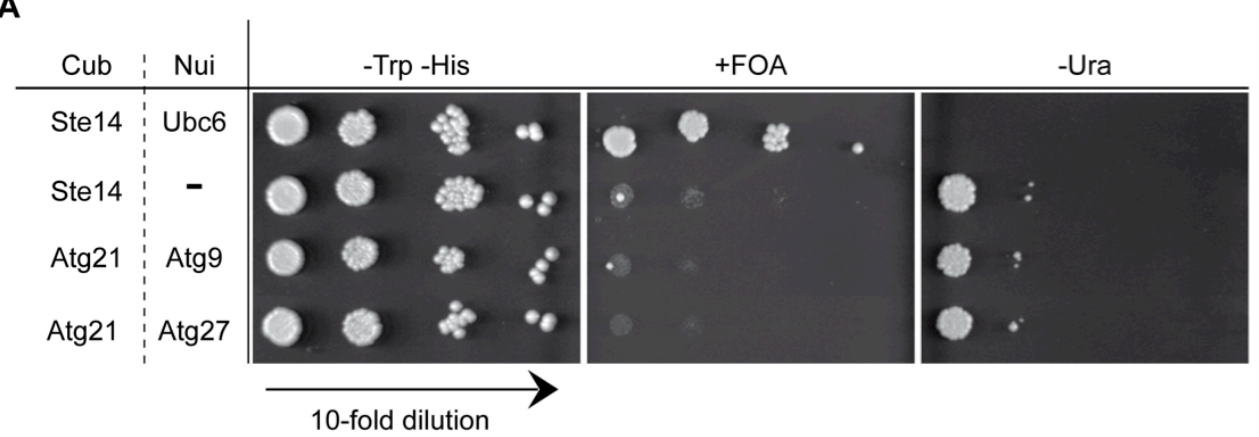

B

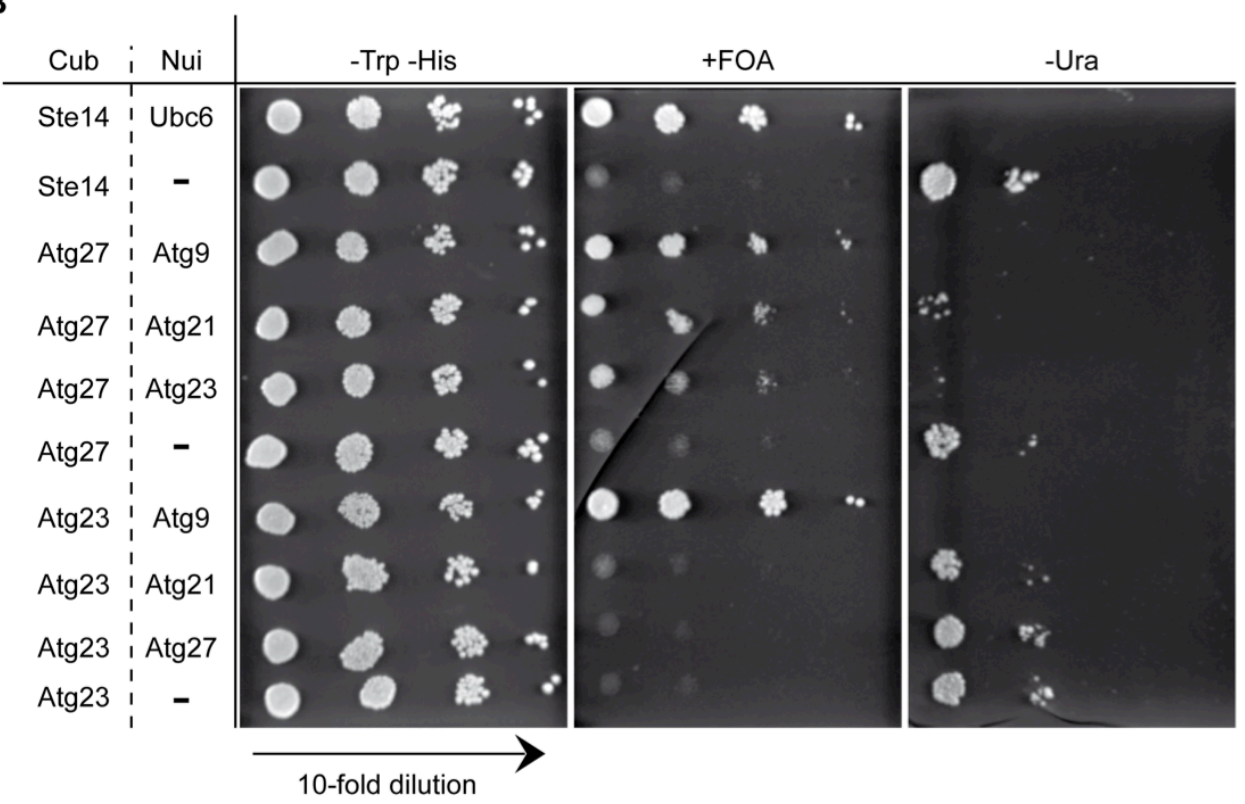

Figure 21: An interaction of Atg27 and Atg21 in split-ubiquitin assay was detected

(A)+(B) $1 \mathrm{OD}_{600}$ WT cells expressing the indicated Cub (bait) and Nui (prey) constructs were diluted in 10-fold steps and spotted on CM-Trp-His (growth control), on CM-Trp-His +FOA (growth implies interaction of the respective proteins) and CM-Trp-His-Ura (growth implies no interaction of the respective proteins). Pictures were taken after three days of growth at $30^{\circ} \mathrm{C}$. Ste14-Cub/Nui-Ubc6: positive control; Ste14-Cub/pRS314: negative control 
Using Atg27-Cub as bait, an interaction with Nui-Atg9, Nui-Atg21 and Nui-Atg23, but not with the empty vector was detected. Atg27 and Atg9 interact directly with each other, resulting in a strong growth on selective medium containing F0A. The interaction with Atg23 was mediated by Atg9 and represented therefore an indirect interaction, resulting in a significantly weaker growth on selective medium containing FOA. Cells expressing Atg27-Cub and Nui-Atg21 showed a medium growth (Figure $21 \mathrm{~B}$ ).

Atg27 consists of 271 amino acids and is a membrane protein type I. Therefore its C-terminal region faces towards the cytosol. Additionally, Atg27 contains a Nterminal signal sequence. Amino acids 199 to 221 represent the transmembrane domain (see Figure 22 A). Amino acid 1 to 198 or amino acid 1 to 221 of Atg27 were fused to the C-terminal half of ubiquitin (Atg271-198-Cub and Atg271-221. Cub) and tested for an interaction with its known interacting partner Nui-Atg9 or -Atg21 (Figure $22 \mathrm{~A}+\mathrm{B})$. 
Results

A

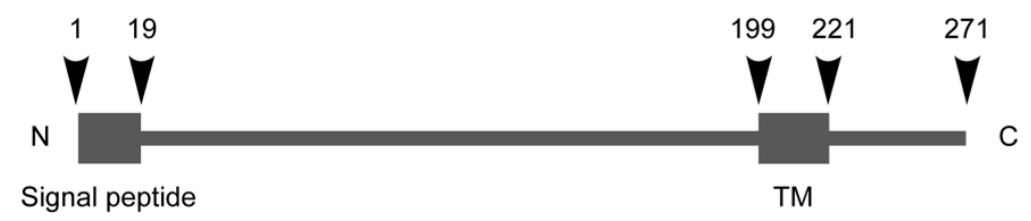

B

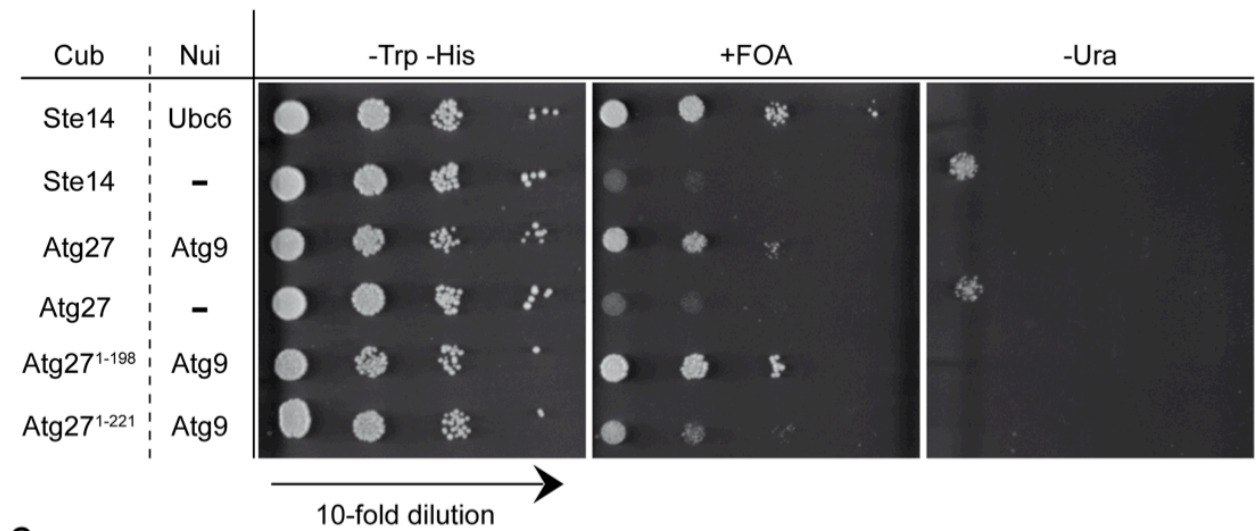

C

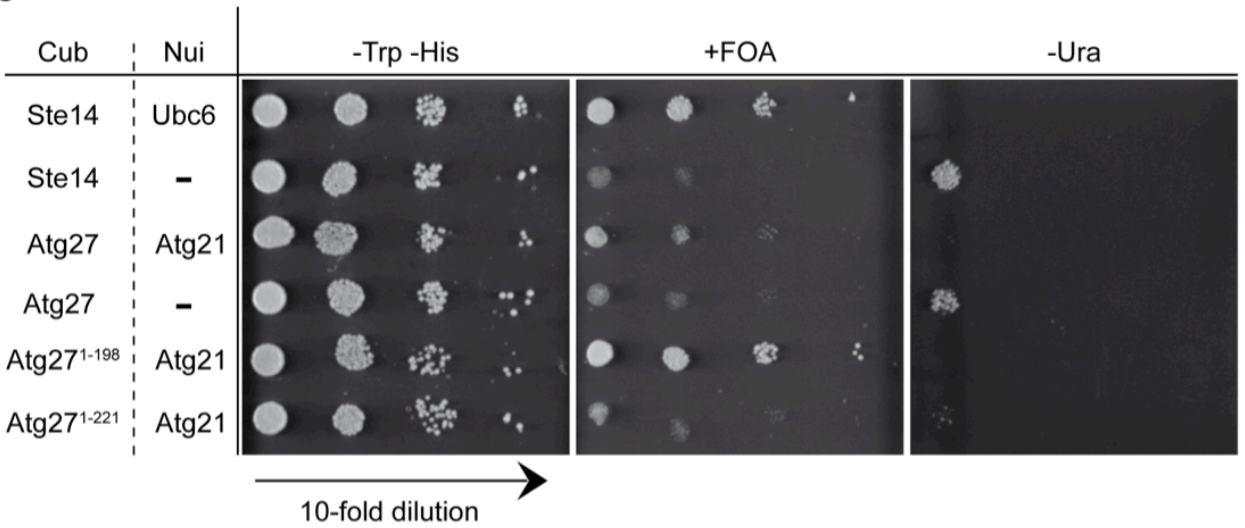

Figure 22: Interaction analysis of truncated Atg27 in split-ubiquitin assay

(A) Predicted domain structure of Atg27 (Yen et al., 2007) (B)+(C) 1 OD $_{600}$ WT cells expressing the indicated Cub (bait) and Nui (prey) constructs were diluted in 10-fold steps and spotted on CM-Trp-His (growth control), on CM-Trp-His +FOA (growth implies interaction of the respective proteins) and CM-Trp-His-Ura (growth implies no interaction of the respective proteins). Pictures were taken after three days of growth at $30^{\circ} \mathrm{C}$. Ste14-Cub/Nui-Ubc6: positive control; Ste14-Cub/pRS314: negative control

The control combination Atg27-Cub and Nui-Atg9 interacted with each other, resulting in a growth on selective medium containing FOA, whereas Atg27-Cub and the empty vector did not. A strong interaction between Atg271-198-Cub, representing only the lumenal part of Atg27, and Nui-Atg9 was detected, as the growth on selective medium containing FOA was improved. In contrast, 
Atg271-221-Cub, additionally containing the transmembrane domain, only weakly interacted with Nui-Atg9 (Figure $22 \mathrm{~A}$ ).

Nearly the same result was obtained for an interaction with Nui-Atg21. Atg27Cub interacted with Nui-Atg21, but not with the empty vector alone, as seen before. An enhanced interaction of Atg271-198-Cub and Nui-Atg21 was detected, whereas Atg271-221-Cub interacted only very weakly with Nui-Atg21 (Figure $22 \mathrm{~B})$.

\subsubsection{Atg21 and Atg27 colocalized in direct fluorescence microscopy}

Legakis et al. (2007) analyzed the Atg23-Atg9-Atg27 complex by direct fluorescence microscopy analysis and showed that approximately $50 \%$ of the Atg9-RFP dots per cell were colocalized with both Atg23-GFP and Atg27-GFP. The same situation was obtained for Atg23-RFP and Atg27-GFP (Legakis et al., 2007). To support a potential interaction of Atg27 and Atg21, both proteins were also analyzed by direct fluorescence microscopy. For this purpose, ATG21 was deleted in a strain, chromosomally expressing Atg27-GFP (cAtg27-GFP). cAtg27GFP was fully functional as it showed wild type ApeI maturation under both growing and starvation conditions (Figure $23 \mathrm{~A}$ ). In cAtg27-GFP atg21 cells the maturation of ApeI was completely blocked under growing conditions and severely impaired under starvation, confirming a successful deletion of ATG21. Wild type and $\operatorname{atg} 1 \Delta$ cells served as a respective positive and negative control (Figure $23 \mathrm{~A}$ ).

cAtg27-GFP atg21 cells were transformed with mCherry-Atg21 expressed from the MET25 promoter, grown to log phase in the presence of $0.3 \mathrm{mM}$ methionine to induce endogenous mCherry-Atg21 expression level, and visualized by direct fluorescence microscopy (Figure 23 B). cAtg27-GFP was visible as several green dots within the cell. As shown before, mCherry-Atg21 formed perivacuolar puncta. 
A

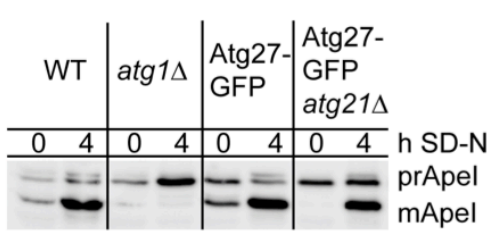

B

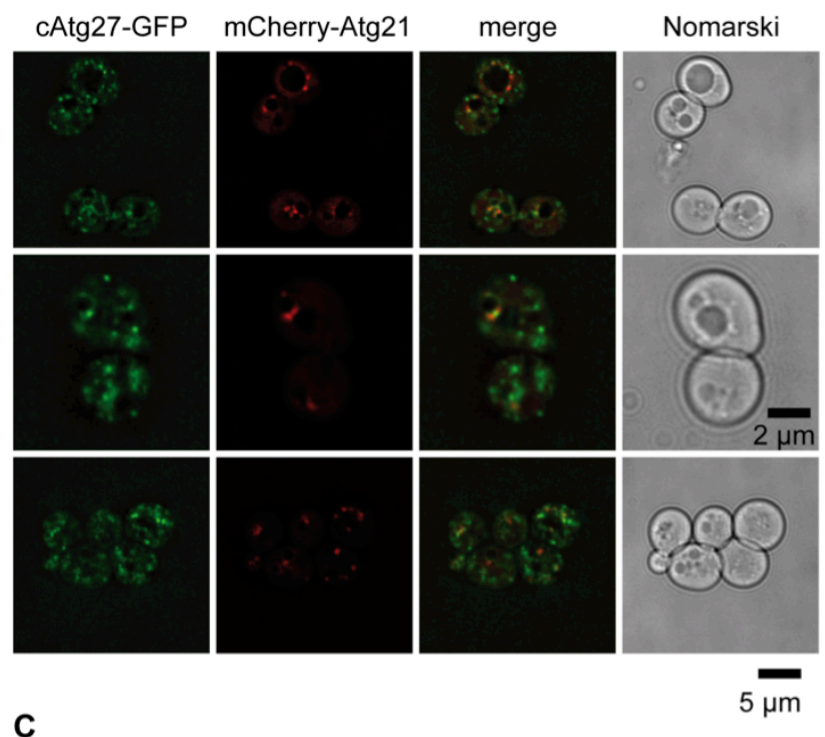

C

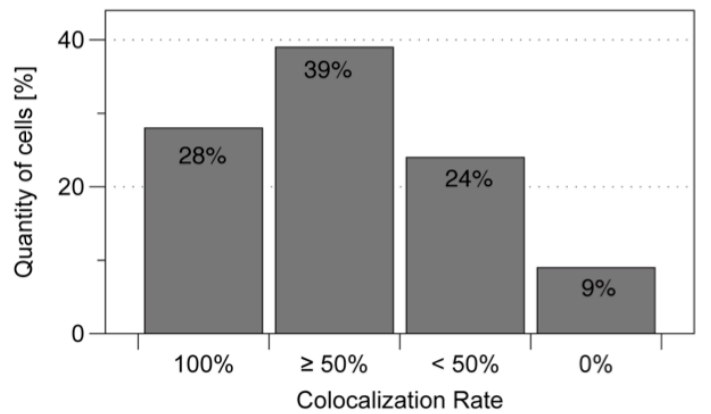

Figure 23: mCherry-Atg21 and Atg27-GFP colocalized in direct fluorescence microscopy

(A) Complementation analysis of chromosomally tagged Atg27-GFP (cAtg27-GFP) in WT and

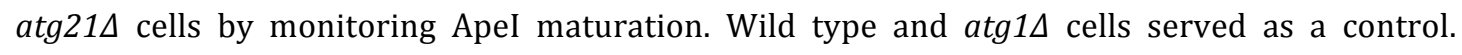
Samples were taken from growing cells $(0 \mathrm{~h})$ and cells starved in SD-N for four hours $(4 \mathrm{~h})$, alkaline lysed and immunoblotted. Precursor (prApeI) and matured ApeI (mApeI) were detected using an anti-ApeI antibody. (B) Growing cAtg27-GFP atg21 cells expressing mCherry-Atg21 from the MET25 promoter were grown to log phase and visualized using a DeltaVision Deconvolution microscope equipped with a TRITC and FITC filter set. Pictures were deconvoluted using SoftWoRx (Applied precision) software. (C) Colocalization rate was quantified by counting the number of mCherry-Atg21 dots that colocalized with cAtg27-GFP per cell $(n=76)$. The cells were sorted into the four indicated categories.

The number of mCherry-Atg21 dots that colocalized with cAtg27-GFP per cell (Colocalization rate) was determined to correlate their expression pattern 
(Figure 23 C). In 28\% of the cells, all mCherry-Atg21 dots were colocalized with Atg27-GFP. At least 50\% colocalization was obtained in 39\% of the counted cells. In $24 \%$ of the cells, less than $50 \%$ of the mCherry-Atg21 dots showed a colocalization with cAtg27-GFP, whereas in only $9 \%$ of the cells, no colocalization of both proteins was detected. Taken together, in more than $50 \%$ of the cells, at least $50 \%$ of mCherry-Atg21 colocalized with cAtg27-GFP, supporting an interaction of both proteins.

\subsubsection{Analysis of the potential interaction of Atg21 and Atg27 by pull down and GFP-TRAP ${ }^{\circledR}$}

To further investigate a potential interaction of Atg21 and Atg27, the binding of Atg27 to Atg21 was examined in pull down experiments and GFP-TRAP ${ }^{\circledR}$ S. For this purpose a chromosomally tagged Atg27-HA (cAtg27-HA) strain was used. As mentioned before, Atg27 has a specific Cvt phenotype (Yen et al., 2007). cAtg27HA showed no defect in ApeI maturation in mid-log phase, suggesting that it was functional (Figure $24 \mathrm{~A}$ ).

For pull down experiments, recombinant 6xHis-SUMO-Atg21 was purified using Nickel-NTA beads. Immobilized 6xHis-SUMO-Atg21 was subsequently incubated with crude extract of log cAtg27-HA yeast cells. After extensive washing, all bound proteins were eluted from the beads, subjected to SDS-PAGE and analyzed by immunoblotting (Figure 24 B). A small part of cAtg27-HA bound specifically to 6 XHis-SUMO-Atg21, but not to 6xHis-SUMO alone, supporting an interaction. Surprisingly, this was not reproducible, as a binding of Atg27-HA to 6xHisSUMO-Atg21 could no longer be obtained in further pull down experiments (data not shown). 
A

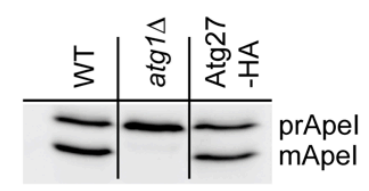

B

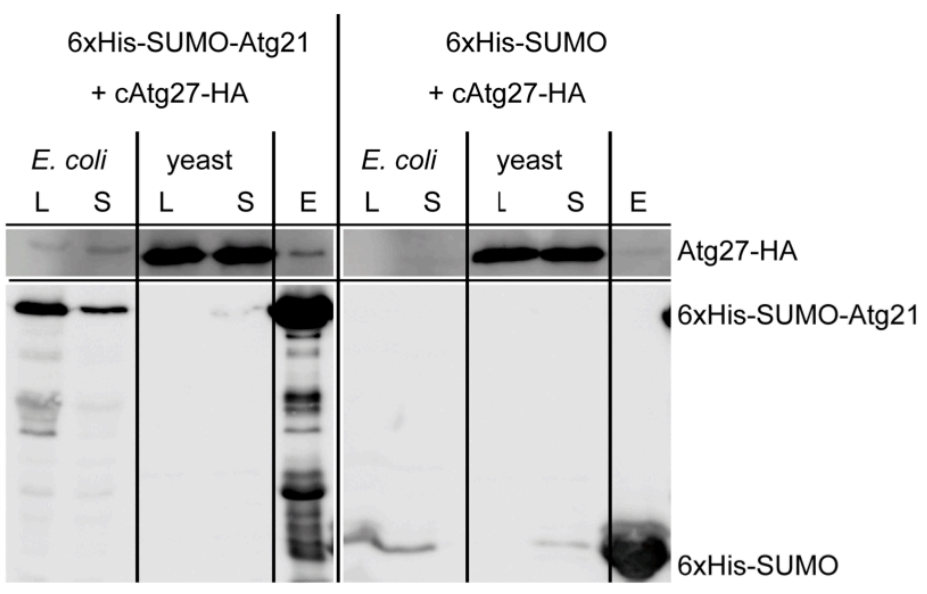

C

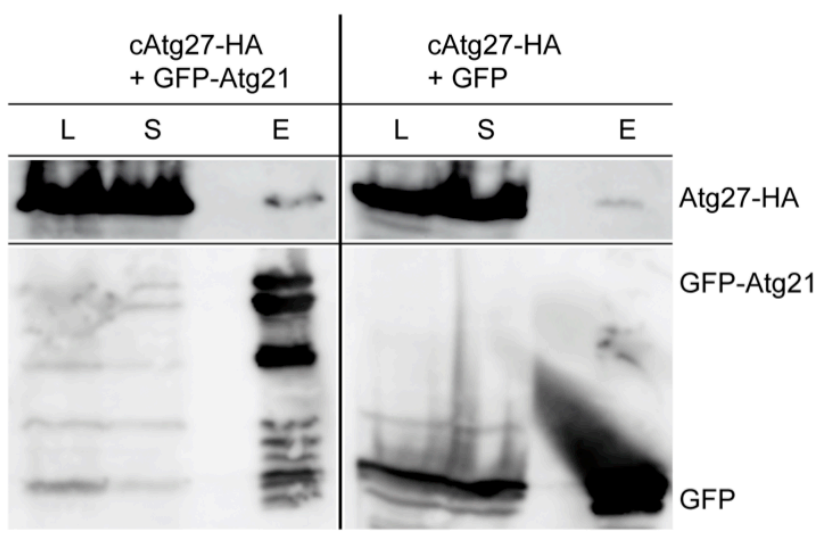

Figure 24: Atg27 does not specifically interact with Atg21 in pull down and GFP-TRAP® ${ }^{\circledR}$ experiments

(A) Complementation study of cAtg27-HA by monitoring Apel maturation. WT, atg1 $\Delta$ and cells chromosomally expressing Atg27-HA (cAtg27-HA) were alkaline lysed in growing phase and immunoblotted. Precursor (prApeI) and matured (mApeI) ApeI were detected using an anti-ApeI antibody. (B) Recombinant 6xHis-SUMO-Atg21 and 6xHis-SUMO alone as negative control were purified using Ni-NTA beads and incubated with crude extract of growing cAtg27-HA cells. Samples from the respective E. coli and yeast lysates (L), cleared lysate (E.coli S), unbound fractions (yeast $S$ ) and the purified proteins (E) were analyzed by immunoblotting using anti-HA and anti-His antibodies. (C) Growing cAtg27-HA cells expressing GFP-Atg21 or GFP alone as negative control were lysed using the mild cell lysis procedure and subjected to GFP-TRAP ${ }^{\circledR}$ analysis. Samples from the cell lysate (L), the unbound fraction (S) and from the purified proteins were analyzed by immunoblotting using anti-HA and anti-GFP antibodies. 
In addition, GFP-TRAP ${ }^{\circledR}$ analysis with growing cAtg27-HA cells expressing GFPAtg21 or GFP alone as control were performed. Here, a part of cAtg27-HA bound to GFP-Atg21, but also to GFP alone (see Figure 24 C). Although the GFP-bound part was apparently smaller than the GFP-Atg21-bound part, an unspecific binding of cAtg27-HA to GFP-Atg21 could not be excluded.

\subsubsection{Interaction studies of Atg21 und Atg8}

Atg8 is one of the core autophagy related proteins. It plays dual roles in autophagy. It is critical for the elongation of the forming autophagosome and it also links the cargo to the autophagosome by direct binding to the cargo receptor during selective types of autophagy. To fulfill its function, Atg8 has to be covalently coupled to PE in a ubiquitin-like manner. Atg8 is synthesized with an additional arginine at its C-terminal end, which is cleaved off by Atg4. Shortened Atg8 is subsequently activated by the E1 enzyme Atg7, transferred to the E2 enzyme Atg3 and finally covalently conjugated to PE by the help of the E3 complex Atg12-Atg5/Atg16 (see chapter 2.2.4.4). Atg21 was shown to be involved in efficient lipidation and in the PAS recruitment of Atg8 (MeilingWesse et al., 2004; Stromhaug et al., 2004).

For the split-ubiquitin assay, Atg8 was only N-terminally fused to the N-terminal half of ubiquitin (Nui-Atg8) because of its C-terminal processing and was tested for an interaction with Atg21-Cub (Figure 25). 

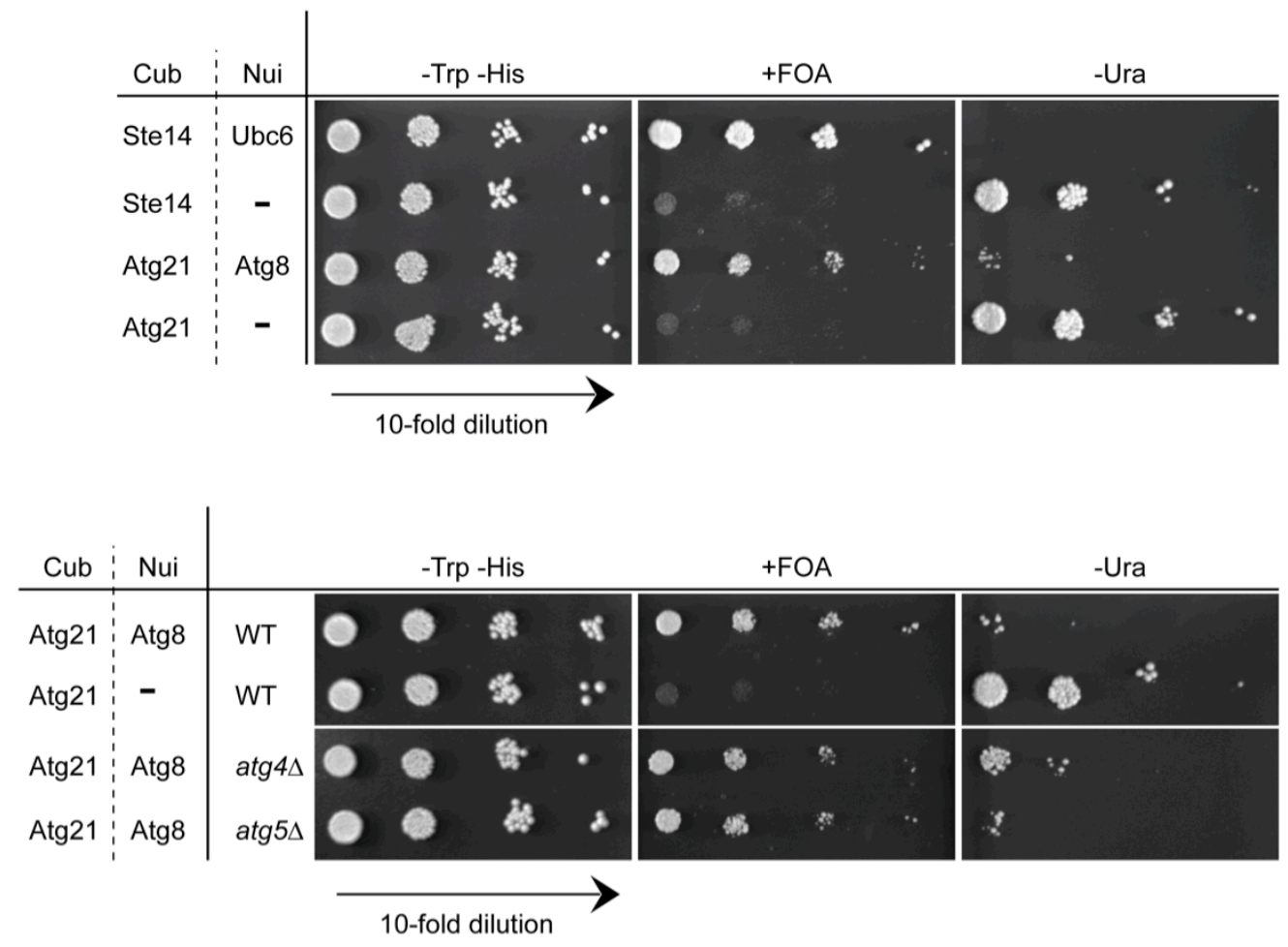

Figure 25: Atg21 and Atg8 interact with each other in split-ubiquitin assay

$1 \mathrm{OD}_{600} \mathrm{WT}$, atg $4 \Delta$ and $\operatorname{atg} 5 \Delta$ cells expressing the indicated Cub (bait) and Nui (prey) constructs were diluted in 10-fold steps and spotted on CM-Trp-His (growth control), on CM-Trp-His +FOA (growth implies interaction of the respective proteins) and CM-Trp-His-Ura (growth implies no interaction of the respective proteins). Pictures were taken after three days at growth at $30^{\circ} \mathrm{C}$. Ste14-Cub/Nui-Ubc6: positive control; Ste14-Cub/pRS314: negative control

Cells expressing both constructs were able to grow on plates containing FOA, but not on plates lacking uracil. In contrast, cells expressing Atg21-Cub and the empty vector (pRS314) showed the opposite growth phenotype and served as an additional negative control (Figure 25). Taken together, this strongly pointed to an interaction of Atg21 and Atg8.

Atg8 exists in two forms within a cell, either unlipidated or covalently conjugated to PE. To test which form interacts with Atg21, the interaction of Atg8 and Atg21 was tested in strains lacking Atg4 or Atg5, where Atg8 lipidation is impaired. In both strains, a clear interaction of Atg21 and Atg8 was detected (Figure 25).

\subsubsection{Bimolecular Fluorescence Complementation (BiFC) analysis}

To further evaluate a possible interaction of Atg8 and Atg21, Bimolecular Fluorescence Complementation (BiFC) analysis was used. For this purpose, two proteins of interest are fused to either the nonfluorescent N-terminal (YN) or C- 
terminal (YC) part of enhanced YFP (eYFP). Both fragments are brought together and are able to reassemble into fluorescent eYFP, if the target proteins interact with each other ((Skarp et al., 2008);Figure 26 A). The interaction of two proteins within the living cell can be easily visualized by fluorescence microscopy. Therefore, it is also possible to determine the interacting site within the cell.

Atg8 was only N-terminally tagged, because it is processed at its C-terminal end. To exclude sterical hindrance, Atg8 was, concerning the split-ubiquitin assay, Nterminally fused to the N-terminal fragment of eYFP (N-YN Atg8) according to the instructions of Skarp et al. (Skarp et al., 2008). Atg21 and Atg4 as a positive control were fused to the corresponding C-terminal fragment (YC) either at the $\mathrm{N}$ - or C-terminus. All constructs were tested for functionality by monitoring ApeI maturation. Wild type and atg1 cells expressing the empty vector were used as a positive and negative control respectively. N-terminally tagged Atg4 (NYC Atg4) was able to restore ApeI maturation in both growing and nitrogen starved atg $4 \Delta$ cells, whereas C-terminally tagged Atg4 (Atg4 C-YC) was not (Figure 26 B). In contrast, C-terminally tagged Atg21 (Atg21 C-YC) complemented the ApeI maturation defect in growing and nitrogen starved $\operatorname{atg} 21 \Delta$ cells, whereas N-terminal tagged Atg21 (N-YC Atg21) did not (Figure $26 \mathrm{~B})$. For this reason N-YCAtg4 and Atg21 C-YC were chosen for further analysis. Interestingly, N-YNAtg8 was able to nearly fully restore ApeI maturation in growing atg8 cells coexpressing C-YC alone (Figure $26 \mathrm{D}$ ). But in growing atg8 cells expressing both N-YN Atg8 and Atg21 C-YC, the ApeI maturation was slightly delayed. 
Results

A
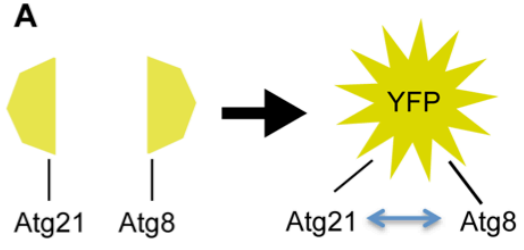

B
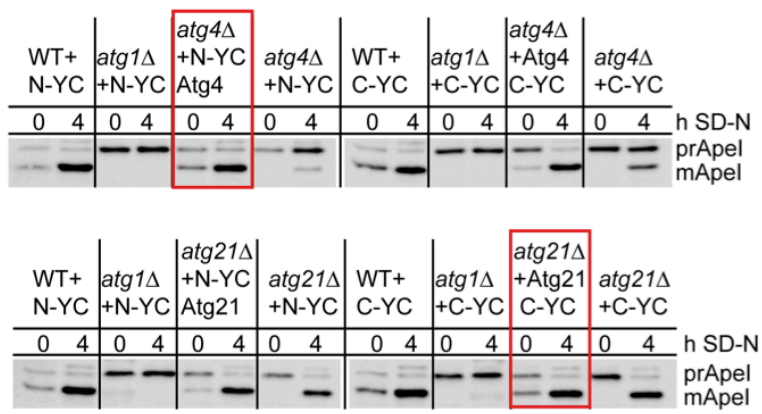

C

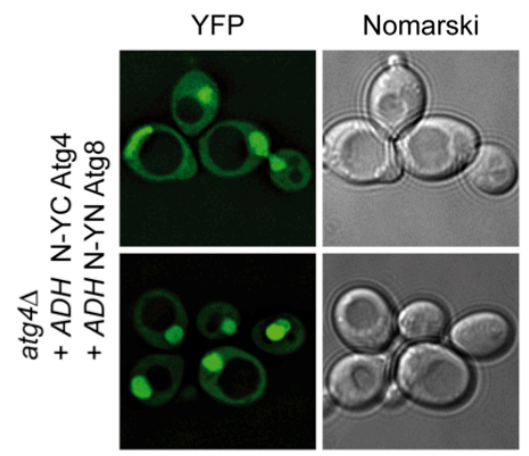

D

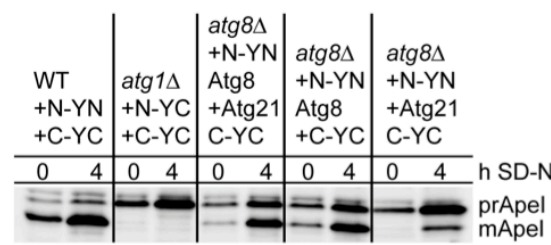

E

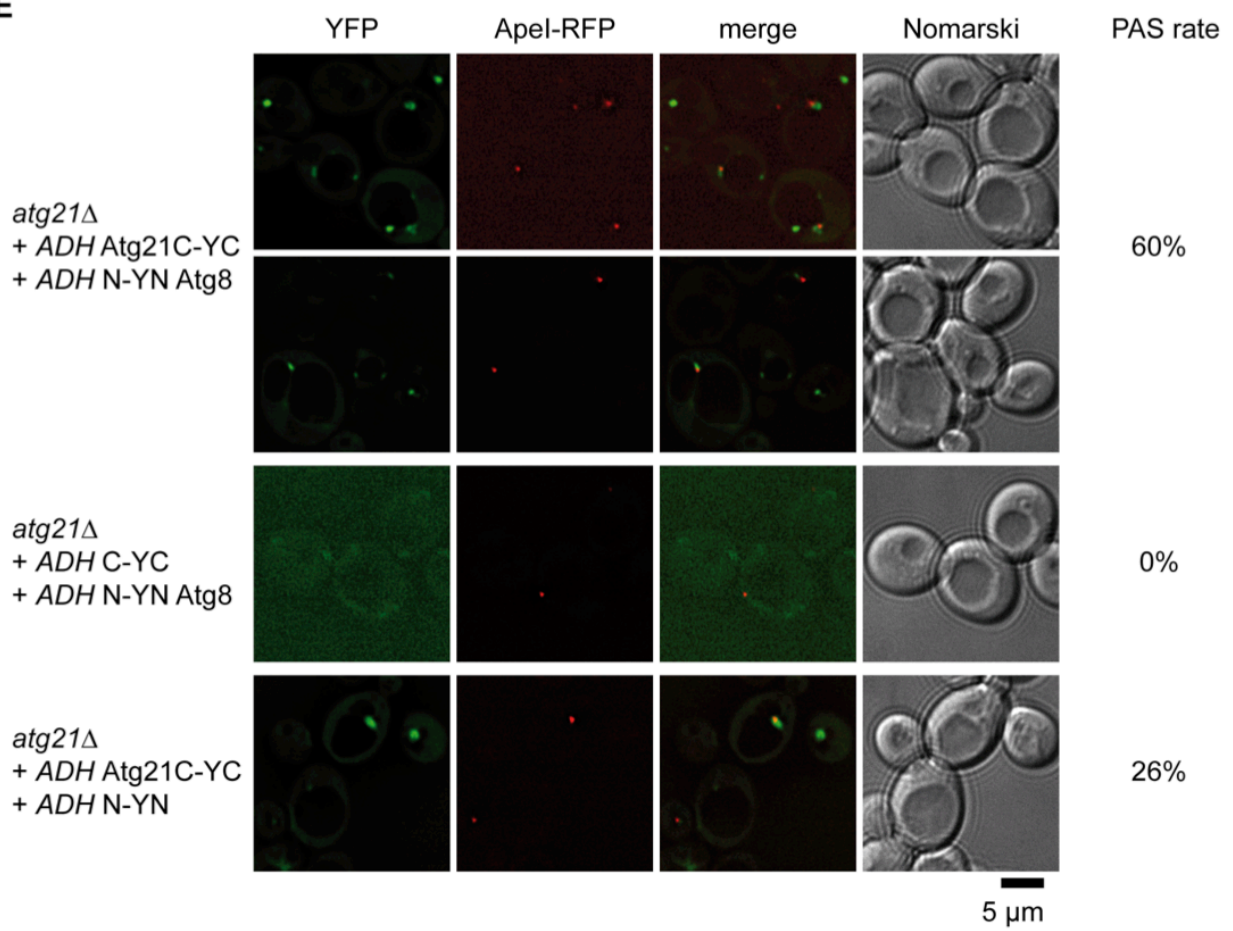

Figure 26: Bimolecular Fluorescence Complementation (BiFC) analysis of Atg8 and Atg21

(A) Scheme of the BiFC analysis. (B) Complementation studies of N-YC Atg4, Atg4 C-YC, N-

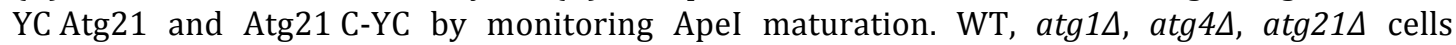
expressing the indicated Atg4 or Atg21 constructs or the respective empty vector were grown to mid-log phase $(0 \mathrm{~h})$ and further starved for four hours in SD-N $(4 \mathrm{~h})$. Samples, taken at the indicated time points, were alkaline lysed and immunoblotted. Precursor (prApeI) and matured ApeI (mApeI) were detected using an anti-ApeI antibody. (C) Growing atg $4 \Delta$ cells expressing NYC Atg4 and N-YN Atg8 from an $A D H$ promoter were visualized using a DeltaVision Deconvolution microscope equipped with a TRITC and FITC filter set. Pictures were 
deconvoluted using SoftWoRx (Applied Precision) software. (D) Complementation studies of NYN Atg8. ApeI maturation was analyzed as described in (B) (E) Growing atg21 cells expressing Atg $21 \mathrm{C}-\mathrm{YC}$ and N-YN Atg8 from a $A D H$ promoter were visualized as described in (C). Both, atg21 cells expressing N-YN Atg8 and C-YC alone or Atg21 C-YC and N-YN alone from an ADH promoter served as negative controls. The PAS rate indicates the number of ApeI-RFP dots that colocalized with an eYFP dot.

First, growing atg $4 \Delta$ cells coexpressing N-YC Atg4 and N-YN Atg8 from the strong and constitutive $A D H$ promoter were analyzed by direct fluorescence microscopy. Since Atg4 and Atg8 interact directly with each other for C-terminal procession of Atg8 (Kirisako et al., 2000; Lang et al., 1998), a clear eYFP signal representing the Atg4-Atg8-eYFP complex, was detected. But unexpectedly, it was exclusively present in the nucleus (Figure $26 \mathrm{C}$ ). A localization of Atg8 and/or Atg4 in the nucleus has not been reported before, suggesting that $\mathrm{N}$ YC Atg4 and N-YN Atg8 were mislocalized.

Next, growing atg21 cells expressing N-YN Atg8 and Atg21 C-YC from the ADH promoter were analyzed by direct fluorescence microscopy. One to five, but intensive green fluorescent dots per cell were detected, strongly suggesting an interaction of Atg8 and Atg21. Since these dots localized at the perivacuolar site, the PAS marker ApeI-RFP was coexpressed to determine if the interaction occurred at the PAS. Indeed, $60 \%$ of the ApeI-RFP dots colocalized with the Atg21-Atg8-eYFP complexes, suggesting that Atg21 and Atg8 interacted with each other at the PAS.

As expected, in the negative control, atg21 cells expressing N-YN Atg8 and C-YC alone from the $A D H$ promoter, no eYFP fluorescence signal could be obtained (Figure 26E). Surprisingly, in the second negative control, atg21 cells expressing Atg21 C-YC and N-YN alone, green fluorescent, perivacuolar dots were detectable, comparable to the signal obtained in cells expressing the respective Atg21 and Atg8 constructs (Figure 26 E). In contrast, the PAS rate of these dots was significantly reduced to only $26 \%$.

The fusion proteins tested so far were highly overexpressed from the constitutive $A D H$ promoter. To avoid overexpression artefacts, a plasmid was constructed, where Atg21 C-YC was expressed from an inducible MET25 promoter. In addition to the regulation of the expression strength, it is possible to induce the expression at a specific time point and therefore to limit the 
expression period of the target protein. To define the appropriate expression level of Atg2 $1 \mathrm{C}-\mathrm{YC}$, that corresponds to the N-YNAtg8 level, $\operatorname{atg} 21 \Delta$ cells carrying the pRS425-ADH-N-YN Atg8 and pUG36-MET25-Atg21 C-YC plasmids were grown in media with different concentrations of methionine. Samples were taken in mid-log phase, alkaline lysed and analyzed by immunoblotting using an anti-GFP antibody (Figure $27 \mathrm{~A}$ ).

A

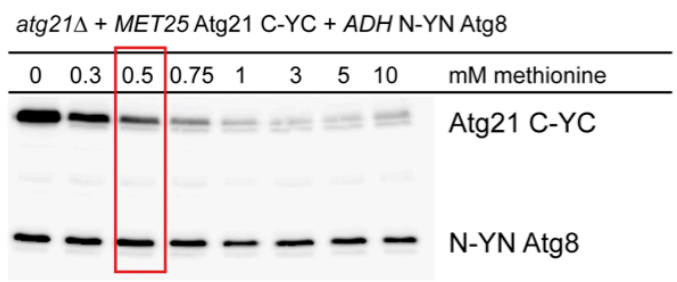

B

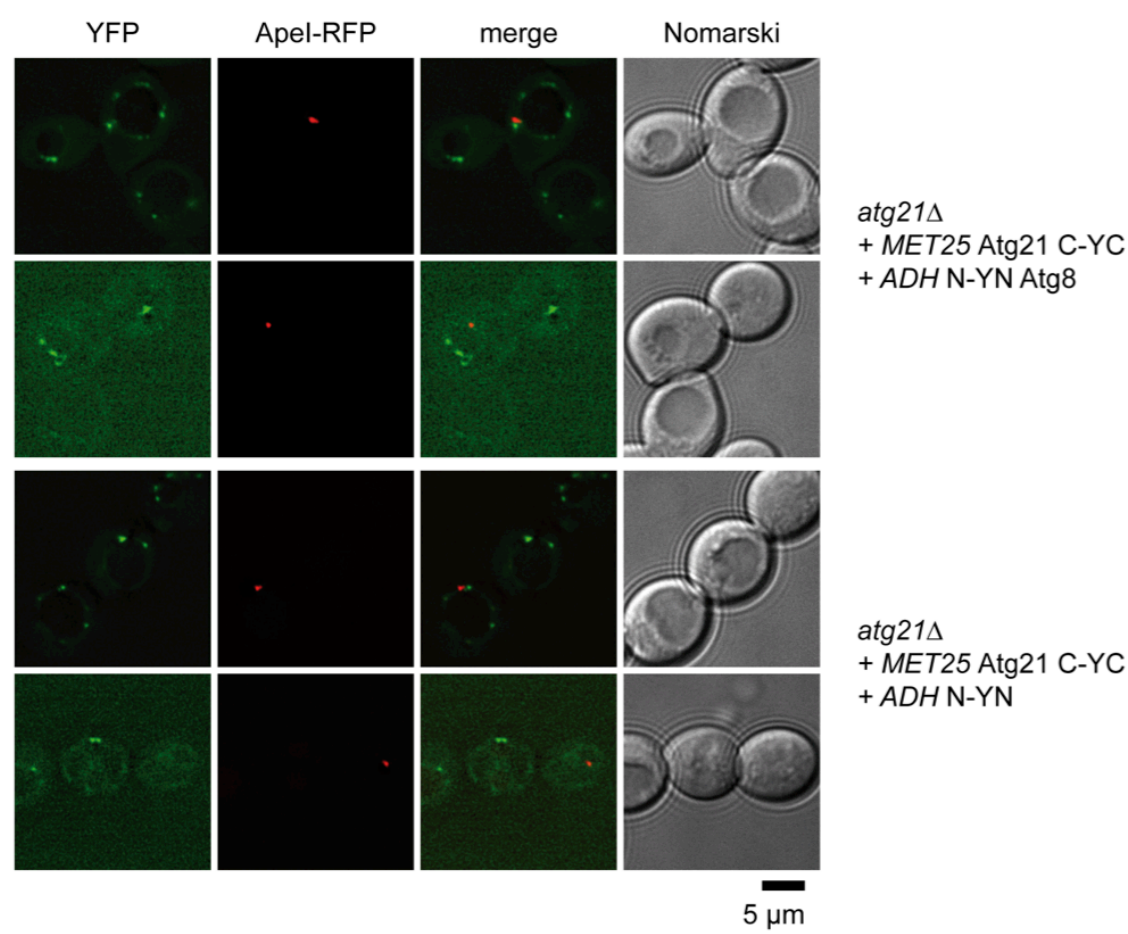

Figure 27: BiFC study of MET25-Atg21 C-YC and ADH-N-YN Atg8

(A) Expression test of MET25-Atg21 C-YC and ADH-N-YN Atg8. Cells were grown in medium containing the indicated methionine concentration to mid-log phase. Samples were alkaline lysed, subjected to immunoblotting and analyzed using an anti-GFP (abcam) antibody. For further studies a concentration of $0.5 \mathrm{mM}$ methionine was chosen. (B) $\operatorname{atg} 21 \Delta$ cells expressing MET25-Atg21 C-YC and ADH-N-YN Atg8 or ADH-N-YN alone were grown in the presence of $0.5 \mathrm{mM}$ methionine to log phase and subsequently visualized using a DeltaVision Deconvolution microscope equipped with a TRITC and FITC filter set. Pictures were deconvoluted using SoftWoRx (Applied Precision) software. 
Since N-YN Atg8 was still expressed from the constitutive $A D H$ promoter, its concentration remained the same independent of the methionine concentration. In contrast, the amount of Atg21 C-YC decreased with increasing methionine concentration. For further approaches, a concentration of $0.5 \mathrm{mM}$ methionine was chosen.

atg214 cells expressing MET25-Atg21 C-YC and ADH-N-YN Atg8 or ADH-N-YN alone were first grown in selective medium containing an excess of methionine to inhibit the expression of Atg2 $1 \mathrm{C}$-YC. Cells were diluted to an $\mathrm{OD}_{600}$ of approximately 0.3 in medium containing $0.5 \mathrm{mM}$ methionine to induce the Atg21 C-YC expression. After cells reached mid-log phase (after three to four hours at $30^{\circ} \mathrm{C}$ ), they were analyzed by direct fluorescence microscopy. Again, in growing atg21 cells expressing MET25-Atg21 C-YC and ADH-N-YN Atg8 one to few perivacuolar, green fluorescent dots per cell were detectable (Figure $27 \mathrm{~B}$ ). In contrast to the conditions used before, the intensity of the dots was much fainter, leading to a greater background noise. Unfortunately, the atg21 $\Delta$ cells expressing MET25-Atg21 C-YC and ADH-N-YN alone showed the same fluorescence signals, pointing again to false positive signals.

As a next step, the amount of N-YNAtg8 was additionally reduced by constructing a plasmid, where N-YN Atg8 was expressed from the MET25 promoter. As before, the expression level of both fusion proteins at different methionine concentrations was tested via immunoblot analysis (Figure $28 \mathrm{~A}$ ). Again, the amount of MET25-Atg21 C-YC decreased with increasing methionine concentration. MET25-N-YN Atg8 was only detectable in the full absence of methionine and therefore at the strongest expression level. This amount was comparable to N-YNAtg8 expressed from the $A D H$ promoter. To avoid overexpression artefacts, a methionine concentration of $0.5 \mathrm{mM}$ was chosen.

As before, atg21 cells expressing Atg21 C-YC and N-YN Atg8 or N-YN alone from the MET25 promoter were grown in the presence of $0.5 \mathrm{mM}$ methionine until log phase and analyzed by direct fluorescence microscopy (Figure 28 B). 
A

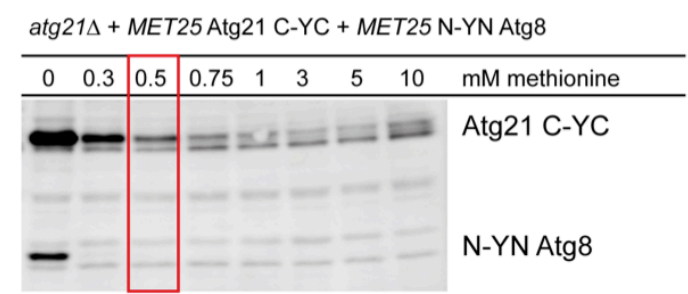

B

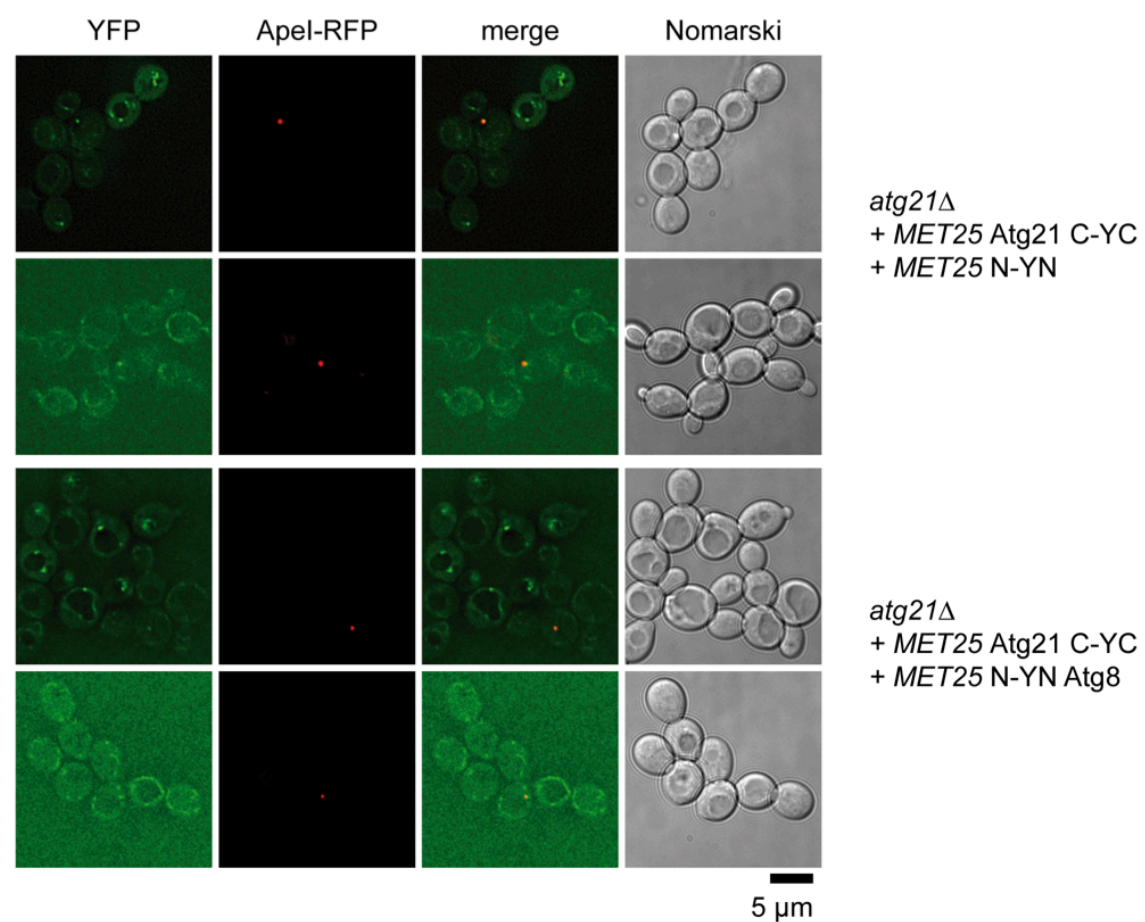

Figure 28: BiFC study of MET25 Atg21 C-YC and MET25 N-YN Atg8

(A) Expression test of MET25 Atg21 C-YC and MET25 N-YN Atg8. Cells were grown in medium containing the indicated methionine concentration to mid-log phase. Samples were alkaline lysed, subjected to immunoblotting and analyzed using an anti-GFP (abcam) antibody. For further studies a concentration of $0.5 \mathrm{mM}$ methionine was chosen. (B) $\operatorname{atg} 21 \Delta$ cells expressing MET25 Atg21 C-YC and MET25 N-YN Atg8 or MET25 N-YN alone were grown in the presence of $0.5 \mathrm{mM}$ methionine to log phase and subsequently visualized using a DeltaVision Deconvolution microscope equipped with a TRITC and FITC filter set. Pictures were deconvoluted using SoftWoRx (Applied Precision) software.

Again, there was no difference in the fluorescence signal in cells expressing both protein constructs or in the negative control expressing only Atg21 C-YC. In further efforts, the methionine concentration, the expression duration and strain background was varied diversely (data not shown). But taken together, all cells 
expressing Atg21 C-YC exhibited a false-positive signal, independent of expression partner, expression level and duration.

\subsubsection{Is Atg8 part of the Atg21 high molecular weight complex detected in gelfiltration?}

Part of Atg21 was detected as a high molecular weight complex in gelfiltration

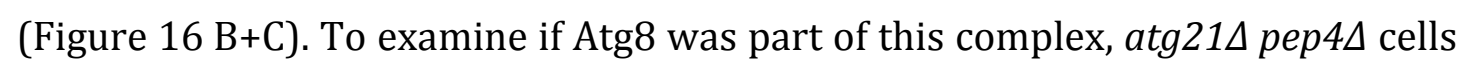
were transformed with pRS313-GFP-Atg8 and pMET25-mCherry-Atg21 or pMET25-mCherry as negative control. $0.3 \mathrm{mM}$ methionine was added to the media to induce endogenous expression level of mCherry-Atg21. The cells were harvested in late growing phase and resuspended in lysis buffer (10 mM MOPS pH 7.2, 0.2 M sorbitol, 1 mM EDTA, 2 mM PMSF, protease inhibitors). Cells were lysed using the mild cell lysis procedure and subjected to gelfiltration analysis as described before (chapter 4.3.1.1).

The main part of mCherry-Atg21 was eluted in fraction 10 and fractions 14 to 15 corresponding to $\geq 670 \mathrm{kDa}$ (Figure $29 \mathrm{~A}$ ). The main part of GFP-Atg8 was eluted in fractions 15 to 17 , and was therefore partly overlapping with the mCherryAtg21 fractions (Figure 29 B). However, the distribution of GFP-Atg8 was not changed in the absence of mCherry-Atg21, indicating, that GFP-Atg8 was not part of the Atg21 high molecular weight complex. Furthermore, GFP-Atg8 has a molecular weight of about $40 \mathrm{kDa}$. The fractions, where GFP-Atg8 was present corresponded to $\geq 65 \mathrm{kDa}$, indicating that GFP-Atg8 was not present in its monomeric form but as an oligomer or in a complex. Nevertheless, since the distribution of GFP-Atg8 was not changed in the absence of mCherry-Atg21, this was independent of Atg21. 
A

$\operatorname{atg} 21 \Delta$ pep $4 \Delta+$ mCherryAtg $21+$ GFP-Atg 8

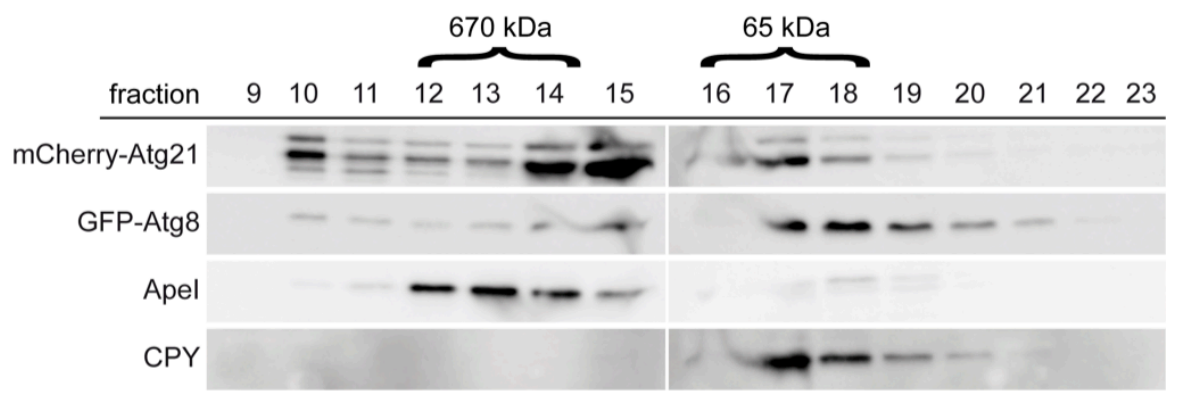

B $\operatorname{atg} 21 \Delta$ pep $4 \Delta+$ mCherry + GFP-Atg8

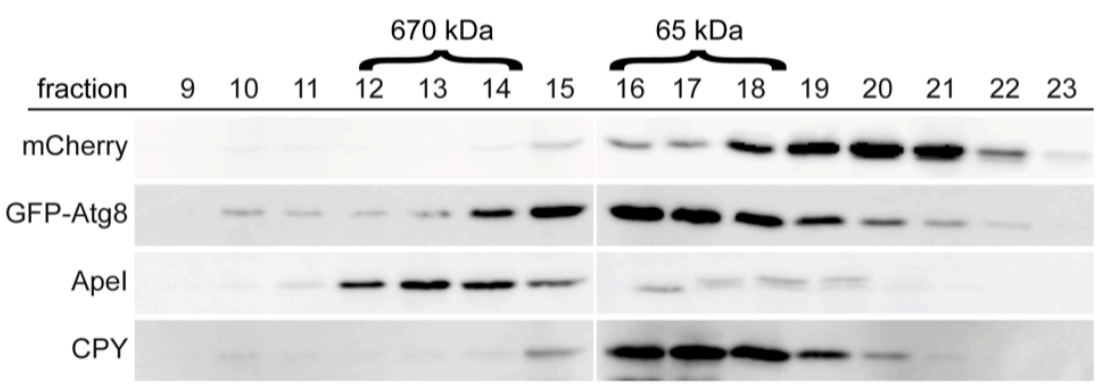

C
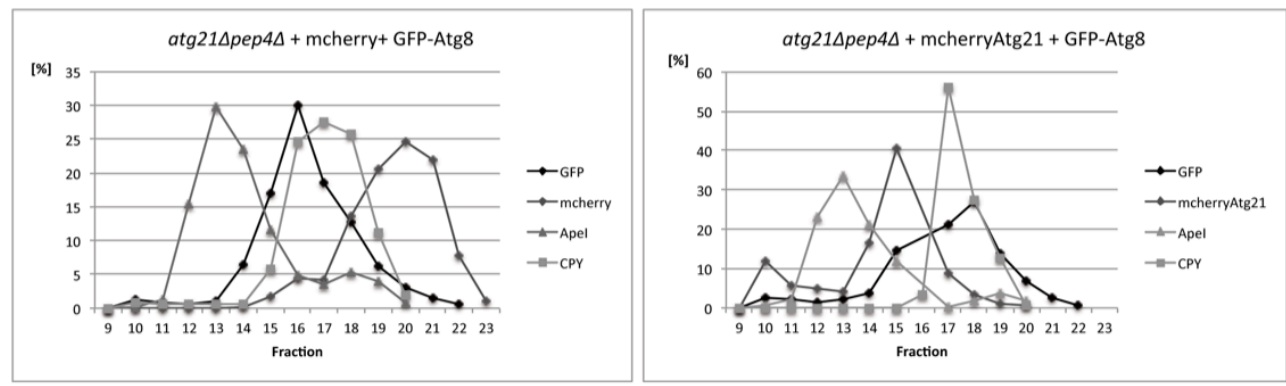

Figure 29: Atg8 distribution in gelfiltration was not changed in the absence of Atg21

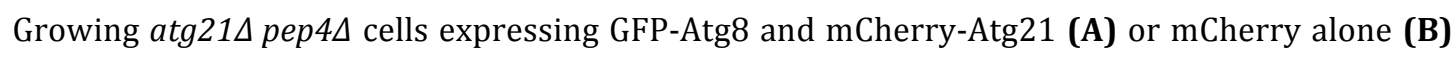
were lysed using the mild cell lysis procedure and subjected to size exclusion chromatography on a Superose 6 column. The cell lysate was eluted from the column at a flow rate of $0.5 \mathrm{ml} / \mathrm{ml}$ and $0.8 \mathrm{ml}$ fractions were collected as it was described in (Obara et al., 2008b). Fractions were precipitated and analyzed by immunoblotting using anti-RED, anti-GFP, anti-ApeI and anti-CPY antibodies. (C) Quantification of the distribution of the individual proteins in the respective fractions using AIDA software. 


\subsubsection{Interaction studies of Atg21 und Atg16}

Atg16 is part of the $A \operatorname{tg} 12-A \operatorname{tg} 5 / A \operatorname{tg} 16$ complex, which acts as an E3-like enzyme in the ubiquitin-like conjugation of Atg8 to PE (Fujita et al., 2008; Hanada et al., 2007).

\subsubsection{Atg21 and Atg16 interact with each other in the split-ubiquitin assay} In the split-ubiquitin assay, Atg21-Cub was tested for an interaction with all members of the Atg12-Atg5/Atg16 complex. Only cells expressing Atg21-Cub and Nui-Atg16 showed a weak growth in the presence of FOA, indicating a weak interaction (Figure $30 \mathrm{~A}$ ). The interaction could be disturbed by steric hindrance and/or interference with endogenous Atg16. To improve the interaction of Atg21 and Atg16, $\operatorname{atg} 16 \Delta$ cells expressing Atg16-Cub and Nui-Atg21 were tested in the split-ubiquitin assay (Figure $30 \mathrm{~B}$ ). 
Results

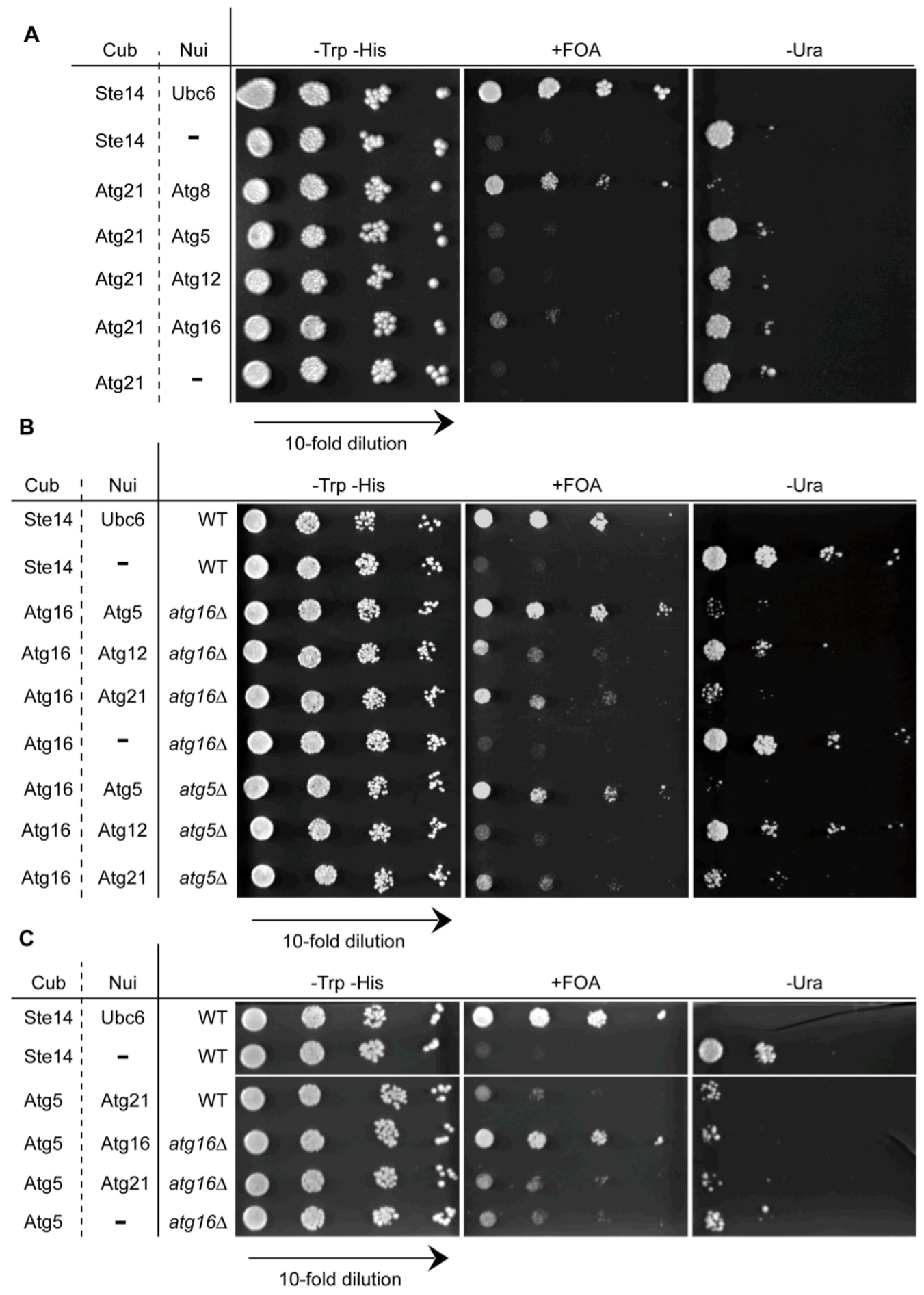

\section{Figure 30: Atg16 and Atg21 interact in the split-ubiquitin assay}

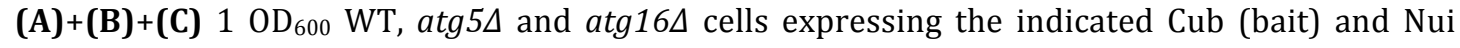
(prey) constructs were diluted in 10-fold dilution steps and spotted on CM-Trp-His (growth control), CM-Trp-His+FOA (growth indicated interaction of the respective proteins) and CM-TrpHis-Ura (growth indicated no interaction of the respective proteins). Ste14-Cub/Nui-Ubc6: positive, Ste14-Cub/pRS314: negative control 
In the E3 complex Atg16 and Atg5 interact directly. Therefore, the Atg16-Atg5 interaction was used as a reference for direct interaction in this analysis. atg16 cells expressing Atg16-Cub and Nui-Atg5 showed a strong growth phenotype in the presence of FOA, whereas the same cells were not able to grow in the absence of uracil. Atg12 and Atg16 interact indirectly via Atg5. Therefore, the combination of Atg16-Cub and Nui-Atg12 resulted in a reduced but still detectable growth on FOA medium. Furthermore, the interaction of Atg16-Cub and Nui-Atg12 could no longer be obtained in atg5 cells. Indeed, a clear interaction of Atg16-Cub and Nui-Atg21 was detected. The growth strength of atg164 cells expressing Atg16-Cub and Nui-Atg21 on FOA medium was significantly improved compared to wild type cells and ranged between the observed direct and indirect control interactions. Furthermore, this interaction was independent of Atg5, as shown in $\operatorname{atg} 5 \Delta$ cells.

To support further that Atg21 interacts specifically with Atg16 and not with another member of the Atg12-Atg5/Atg16 complex, Atg5 and Atg21 were further tested using the split-ubiquitin assay (Figure $30 \mathrm{C}$ ). No interaction of Atg5-Cub and Nui-Atg21 was observed, either in WT or in atg164 cells. In contrast, Atg5Cub and Nui-Atg16 interacted with each other as expected, confirming the functionality of Atg5-Cub.

In conclusion, the split-ubiquitin assay pointed to an interaction of Atg21 with Atg16, but with no other member of the Atg12-Atg5/Atg16 complex.

\subsubsection{Fluorescence microscopy analysis of Atg5 and Atg16}

Atg5 and Atg16 are only transiently located at the PAS and therefore hardly detectable in fluorescence microscopy. Stromhaug et al. (2004) showed, that the PAS localization of Atg5-GFP can be enhanced in mutants defective in the progression of the Cvt / autophagy pathways as atg84 cells. Furthermore, the PAS localization of Atg5-GFP observed in atg8 cells was prevented in the additional absence of Atg21 (Stromhaug et al., 2004). Regarding Atg16, Nair et al. (2010) showed that the perivacuolar puncta formation of chromosomally tagged Atg16-GFP was significantly decreased in starved atg18 $\operatorname{atg} 21 \Delta$ cells. Since Atg21 is required for the Cvt pathway, which takes place under growing conditions, the PAS localization of Atg16 was observed in growing cells. As a 
prerequisite for further successful colocalization studies of Atg5 and Atg16, the observations reported by Stromhaug et al. (2004) should be reproduced. For this purpose, atg8 8 and $\operatorname{atg} 8 \Delta \operatorname{atg} 21 \Delta$ cells expressing Atg5-YFP and the PAS marker ApeI-RFP were grown to mid-log phase and visualized using a DeltaVision fluorescence microscope (Figure $31 \mathrm{~A}$ ).

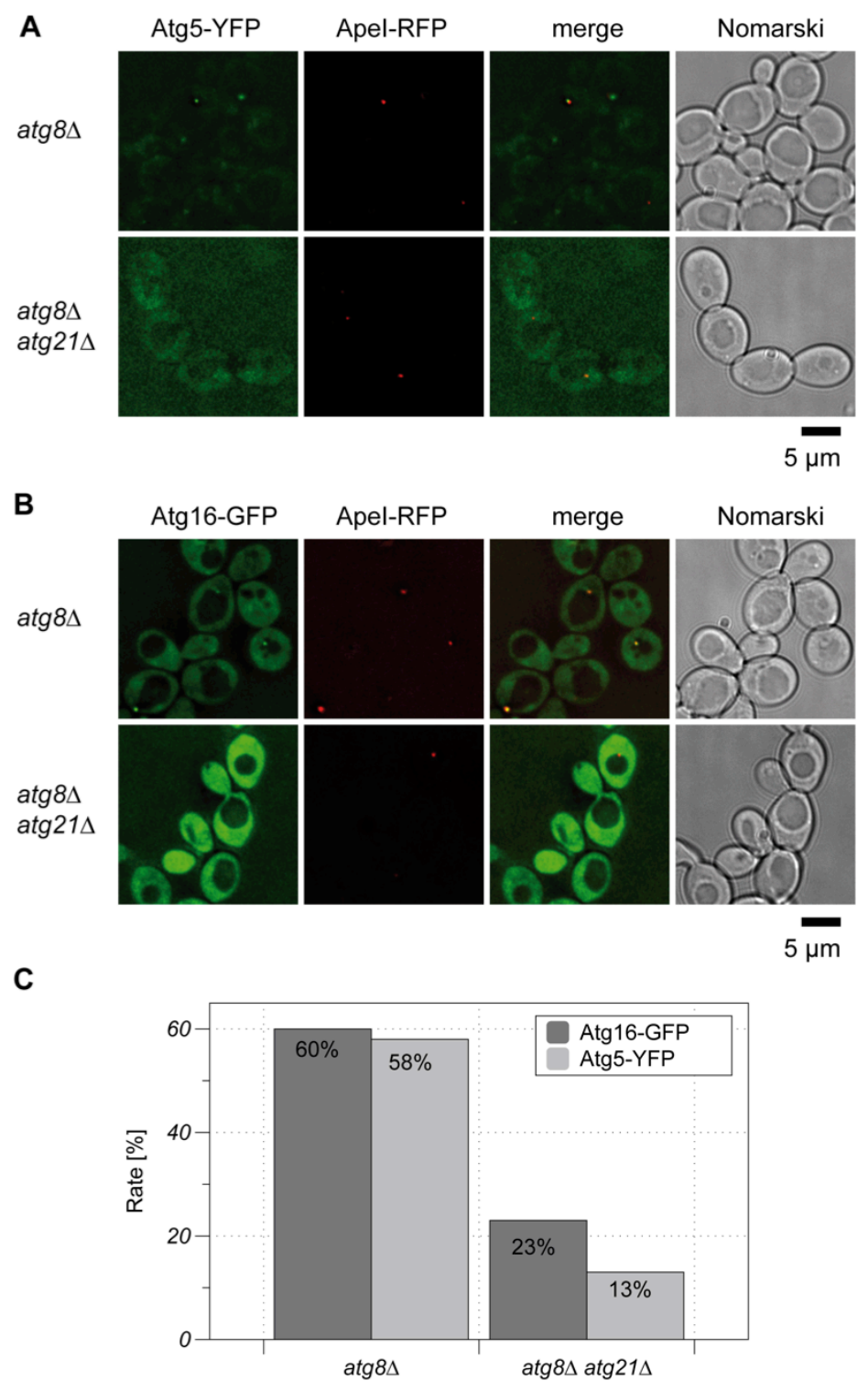

Figure 31: Atg5 and Atg16 are absent from the PAS in growing atg84 atg214 cells

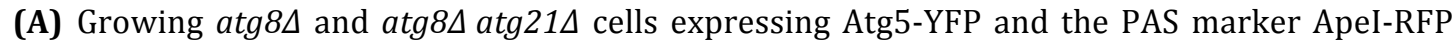
were visualized using a DeltaVision Deconvolution microscope equipped with YFP and RFP filter sets. Pictures were deconvoluted using SoftWoRx (Applied Precision) software. (B) Growing

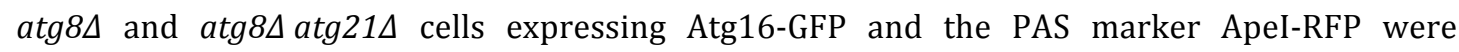
visualized as described in (A). (C) The percentage of cells showing a perivacuolar Atg5-YFP respective Atg16-GFP puncta was determined for each strain. 
A clear perivacuolar Atg5-YFP punctum was observed in 58\% of the atg $8 \Delta$ cells. Nearly each of these puncta was colocalized with the PAS marker ApeI-RFP. In contrast, only $13 \%$ of the atg $8 \Delta$ atg $21 \Delta$ cells showed a much fainter perivacuolar Atg5-YFP punctum (Figure $31 \mathrm{~A}+\mathrm{C}$ ). These observations correlated perfectly with the data obtained by Stromhaug et al. (2004), confirming the functionality of this assay. Next, atg8 $\Delta$ and $\operatorname{atg} 8 \Delta \operatorname{atg} 21 \Delta$ cells expressing Atg16-GFP and the PAS marker ApeI-RFP were grown to log phase and visualized by direct fluorescence microscopy. In $60 \%$ of the $\operatorname{atg} 8 \Delta$ cells, a clear perivacuolar Atg16GFP punctum was observed (Figure $31 \mathrm{~B}+\mathrm{C}$ ). All these puncta colocalized with the PAS marker ApeI-RFP. The PAS localization of Atg16-GFP could therefore also be enhanced in mutants defective in the progression of the Cvt / autophagy pathways as published for Atg5. Furthermore, the PAS localization of Atg16-GFP observed in atg8 cells was significantly reduced by additional deletion of ATG21, since only $23 \%$ of $\operatorname{atg} 8 \Delta \operatorname{atg} 21 \Delta$ cells showed a perivacuolar Atg16-GFP punctum (Figure $31 \mathrm{~B}+\mathrm{C}$ ). Again, these puncta were much fainter, compared to those observed in atg8 cells. In conclusion, both Atg5-YFP and Atg16-GFP were absent from the PAS in growing atg $8 \Delta \operatorname{atg} 21 \Delta$ cells.

Additionally, the colocalization of Atg5-YFP and Atg16-GFP with mCherry-Atg21 was determined in different mutant strains (Figure $32 \mathrm{~A}+\mathrm{B}$ ). $0.3 \mathrm{mM}$ methionine was added to the selective medium to induce endogenous expression of mCherry-Atg21. 
Results

A
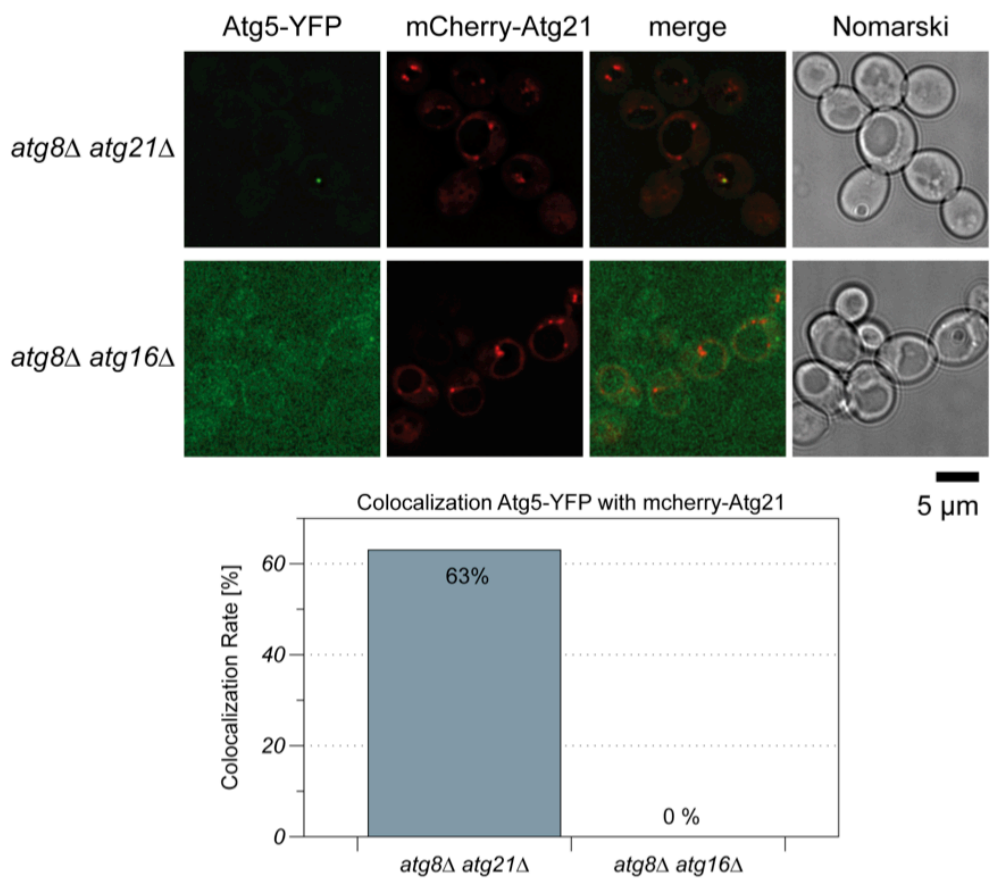

$5 \mu \mathrm{m}$

B
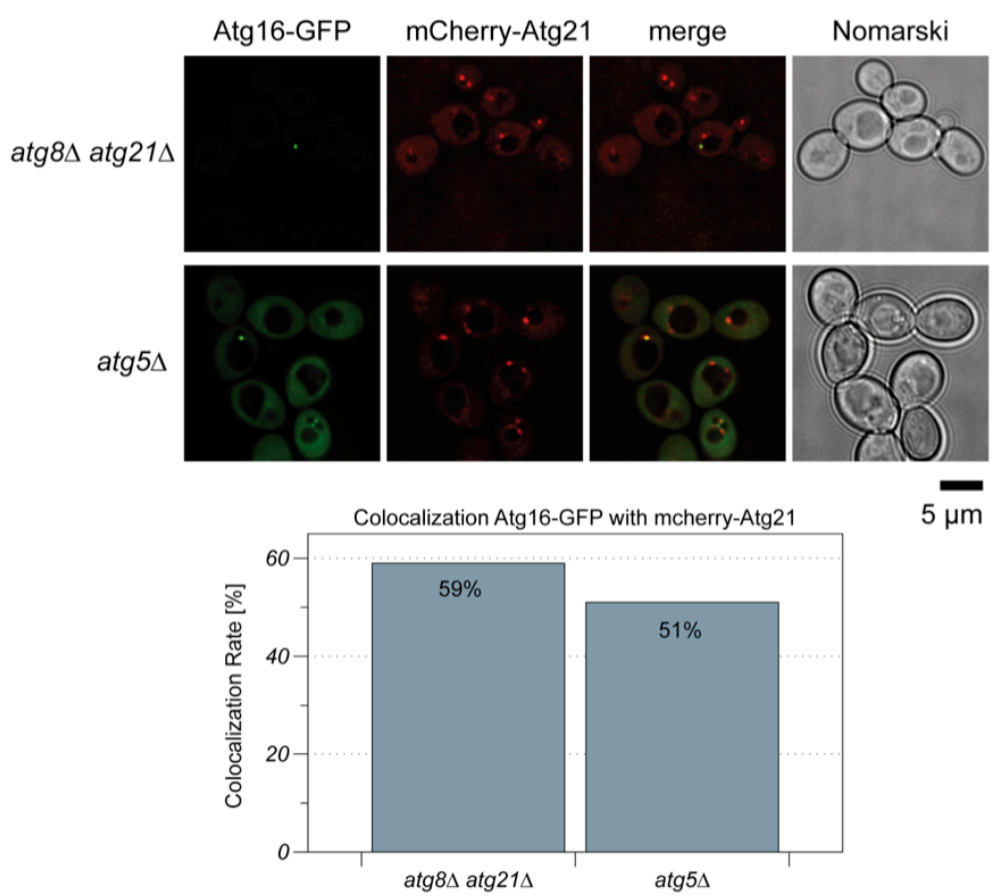

Figure 32: Atg16 and Atg21 colocalize in the absence of Atg5

(A) $\operatorname{atg} 8 \Delta \operatorname{atg} 21 \Delta$ and $\operatorname{atg} 8 \Delta \operatorname{atg} 16 \Delta$ cells expressing Atg5-YFP and mCherry-Atg21 were grown to mid-log phase in the presence of $0.3 \mathrm{mM}$ methionine and visualized using a DeltaVision Deconvolution microscope equipped with YFP and mCherry filter sets. Pictures were deconvoluted using SoftWoRx (Applied Precision) software. The percentage of Atg5-YFP colocalized with mCherry-Atg21 was determined. (B) $\operatorname{atg} 8 \Delta \operatorname{atg} 21 \Delta$ and $\operatorname{atg} 5 \Delta$ cells expressing Atg16-GFP and mCherry-Atg21 were grown to log phase in the presence of $0.3 \mathrm{mM}$ methionine and visualized using a DeltaVision Deconvolution microscope equipped with GFP and mCherry 
filter sets. Pictures were deconvoluted using SoftWoRx (Applied Precision) software. The percentage of Atg16-GFP colocalized with mCherry-Atg21 was determined.

In mid-log atg $8 \Delta \operatorname{atg} 21 \Delta$ cells $63 \%$ of the perivacuolar Atg5-YFP puncta and 59\% of the perivacuolar Atg16-GFP puncta were colocalized with mCherry-Atg21 (Figure $32 \mathrm{~A}+\mathrm{B}$ ). mCherry-Atg21 most likely colocalized with both proteins at the PAS, because all of the Atg16-GFP and nearly all of the Atg5-YFP dots was located at the PAS under the same conditions (Figure 31). Atg16 links the Atg12Atg5/Atg16 complex to the PAS (Matsushita et al., 2007). Therefore, Atg5-YFP

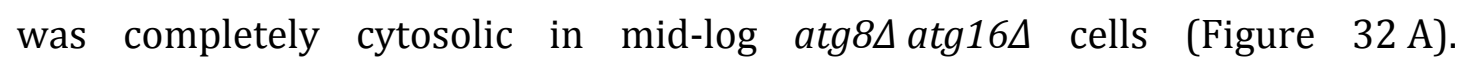
Importantly, the colocalization rate of Atg16-GFP and mCherry-Atg21 did not change in the absence of Atg5 (Figure $32 \mathrm{~B}$ ).

\subsubsection{Interaction studies of Atg16 and Atg21 using pull down and GFP- TRAP ${ }^{\circledR}$ analysis}

To further confirm a potential interaction of Atg21 and Atg16, pull down and GFP-TRAP ${ }^{\circledR}$ analysis were performed using chromosomally tagged Atg16-HA (cAtg16-HA). cAtg16-HA was still able to mature ApeI under growing conditions, indicating that it was functional (Figure $33 \mathrm{~A}$ ).

For pull down experiments, recombinant 6xHis-SUMO-Atg21 and 6xHis-SUMO alone as a control were purified using Ni-NTA beads and subsequently incubated with crude extract of growing cAtg16-HA cells. After extended washing, the isolated proteins were eluted from the beads by adding Laemmli buffer containing $100 \mathrm{mM}$ DTT. cAtg16-HA bound specifically to 6xHis-SUMO-Atg21 but not to 6xHis-SUMO alone (see Figure $33 \mathrm{~B}$ ).

For GFP-TRAP ${ }^{\circledR}$ analysis, growing cAtg16-HA cells expressing GFP-Atg21 or GFP alone as negative control, were lysed using the mild cell lysis procedure. The cell lysates were subjected to GFP-TRAP ${ }^{\circledR}$ beads. After extended washing, the purified proteins were eluted by the addition of Laemmli buffer. cAtg16-HA was specifically co-isolated by GFP-Atg21, but not by GFP alone (Figure 33 C). Atg16 is a member of the E3-like enzyme complex crucial for the lipidation of Atg8. As shown before, Atg21 interacted with Atg8 in the split-ubiquitin assay (see chapter 4.3.5). To exclude the possibility, that Atg16 was co-precipitated by a bridging interaction of Atg8, a GFP-TRAP ${ }^{\circledR}$ analysis was performed with cell 
lysate of cAtg16-HA atg84 cells expressing GFP-Atg21 or GFP alone as negative control. Indeed, also in the absence of Atg8, cAtg16-HA was specifically coisolated by GFP-Atg21, but not by GFP alone (Figure $33 \mathrm{C}$ ).

A

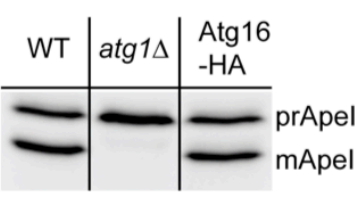

B

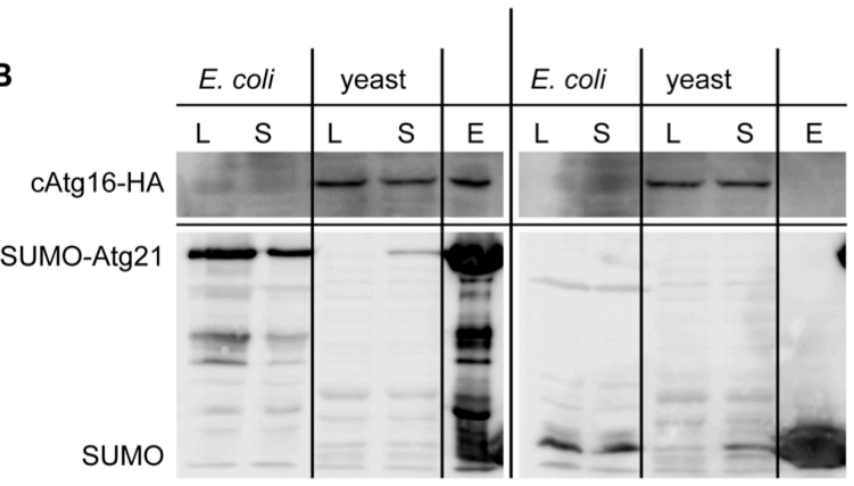

C

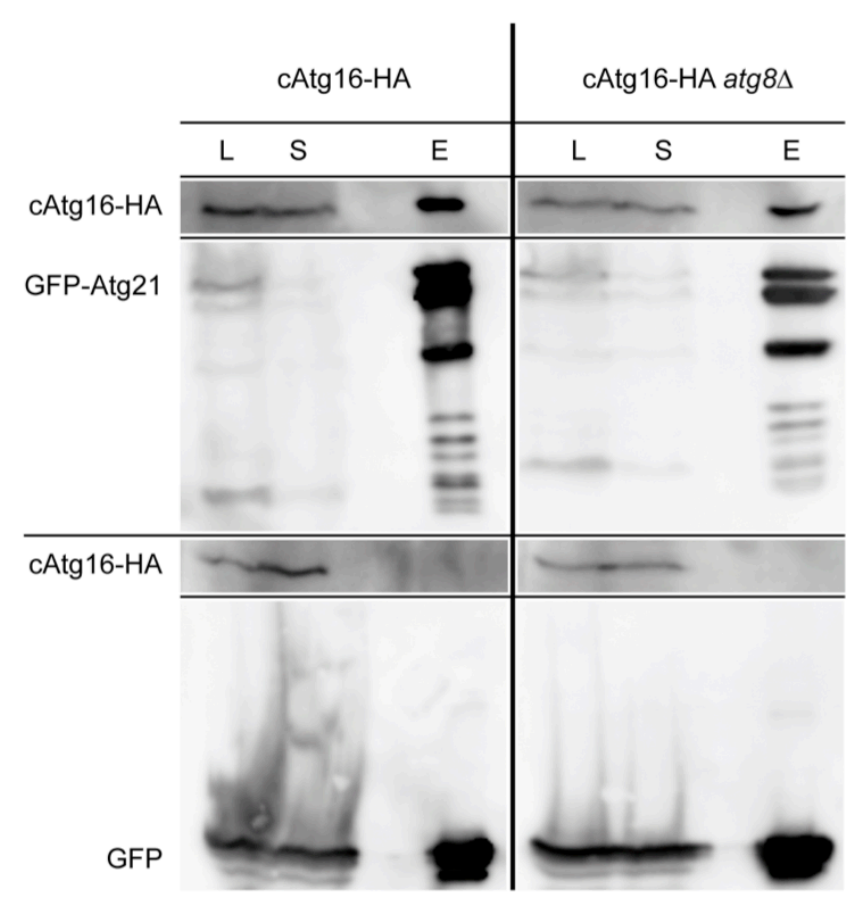

D

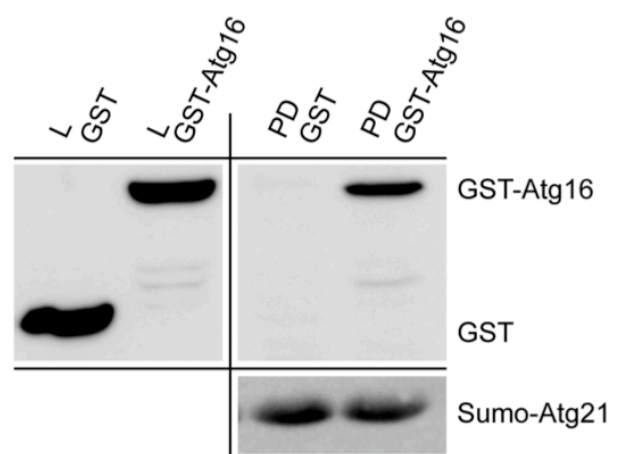

Figure 33: Interaction studies of Atg16 and Atg21 using GFP-TRAP ${ }^{\circledR}$ and pull down experiments

(A) Complementation study of chromosomally tagged Atg16-HA (cAtg16-HA) by monitoring the maturation of ApeI. Samples from growing WT, atg1 14 and cAtg16-HA cells were alkaline lysed and immunoblotted. Precursor (prApeI) and matured (mApeI) ApeI were detected using an anti- 
ApeI antibody. (B) Recombinant 6xHis-SUMO-Atg21 and 6xHis-SUMO alone as a negative control were purified using Ni-NTA beads and incubated with crude extract of growing cAtg16-HA cells. Samples from the respective E. coli and yeast cell lysates (L), cleared lysate (E. coli S), unbound fraction (yeast $S$ ) and the purified proteins (E) were analyzed by immunoblotting using anti-HA and anti-His antibodies. (C) Growing cAtg16-HA cells or cAtg16-HA atg84 cells expressing GFPAtg21 or GFP as negative control were lysed using the mild cell lysis procedure and subjected to GFP-TRAP ${ }^{\circledR}$ analysis. Samples from the cell lysate (L), the unbound fraction (S) and from the purified proteins (E) were analyzed by immunoblotting using anti-HA and anti-GFP antibodies. (D) Immobilized recombinant 6xHis-SUMO-Atg21 was incubated with purified recombinant GST and GST-Atg16. Samples from purified GST-Atg16 and GST (L) and samples from the eluted proteins (PD) were immunoblotted and detected using anti-GST and anti-His antibodies.

To further confirm a direct interaction of Atg16 and Atg21, a direct pull down experiment using purified recombinant 6xHis-SUMO-Atg21 and GST-Atg16 were performed. For this purpose, recombinant GST-Atg16 and GST alone as a negative control were purified using glutathione sepharose. After extended washing, GST and GST-Atg16 were eluted by addition of glutathione. Twice the amount was incubated with immobilized 6xHis-SUMO-Atg21. After extended washing, the bound proteins were eluated by adding Laemmli buffer and analyzed by immunoblot (Figure $33 \mathrm{D}$ ). In fact, GST-Atg16 bound specifically to 6xHis-SUMO-Atg21, whereas GST alone did not.

In conclusion, Atg21 probably directly interacts with Atg16.

\subsubsection{Determination of the Atg21- interacting domain of Atg16}

Atg16 consists of 150 amino acids. Amino acids 22 to 46 are predicted to constitute the Atg5-binding region, followed by a short linker region. Amino acids 58 to 119 form a coiled-coil domain, which mediates dimerization of Atg16 ((Fujioka et al., 2010);Figure 34 A).

To identify the Atg21-interacting domain of Atg16, truncated GST-Atg16 versions were constructed and tested for an interaction with 6xHis-SUMO-Atg21 in direct pull down experiments as described before. For this purpose, recombinant GST-Atg16 lacking the coiled-coil domain (GST-Atg16aa1-57), lacking the extreme C-terminal end (GST-Atg16 aa1-119), which is not required for the coiled-coil formation or lacking the Atg5-binding domain and the following linker region (GST-Atg16 ${ }^{\text {aa58-150) }}$ were purified and incubated with recombinant 
6xHis-SUMO-Atg21. GST-Atg16 and GST-Atg16aa58-150 bound specifically to 6xHis-SUMO-Atg21, whereas GST alone, GST-Atg16 aa1-57 and GST-Atg16 $6^{\text {aa1-119 }}$ did not (Figure $34 \mathrm{~B})$.

The same truncated versions of Atg16 were created as Atg16-HA constructs and cloned into a pRS313 vector under the control of a CUP1 promoter. Wild type Atg16-HA and the truncated Atg16-HA versions were co-expressed with GFPAtg21 or GFP alone as negative control in atg16 cells. The amount of Atg161-119_ HA and Atg16 68-150-HA or Atg161-57-HA was adjusted by addition of $25 \mu \mathrm{M} \mathrm{CuSO}_{4}$ or $50 \mu \mathrm{M} \mathrm{CuSO}_{4}$ to the selective medium over night (approximately 12 hours), respectively. Cells were grown to mid-log phase, lysed under mild conditions and subjected to GFP-TRAP ${ }^{\circledR}$ analysis (Figure $34 \mathrm{C}$ ).

A

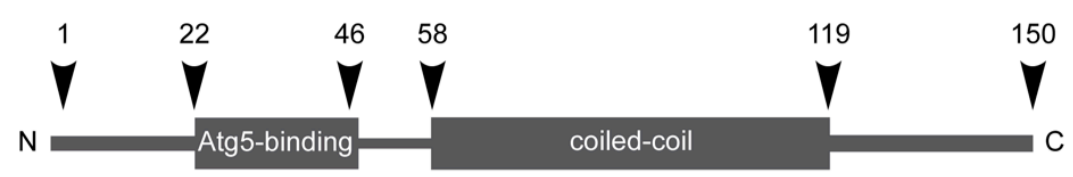

B

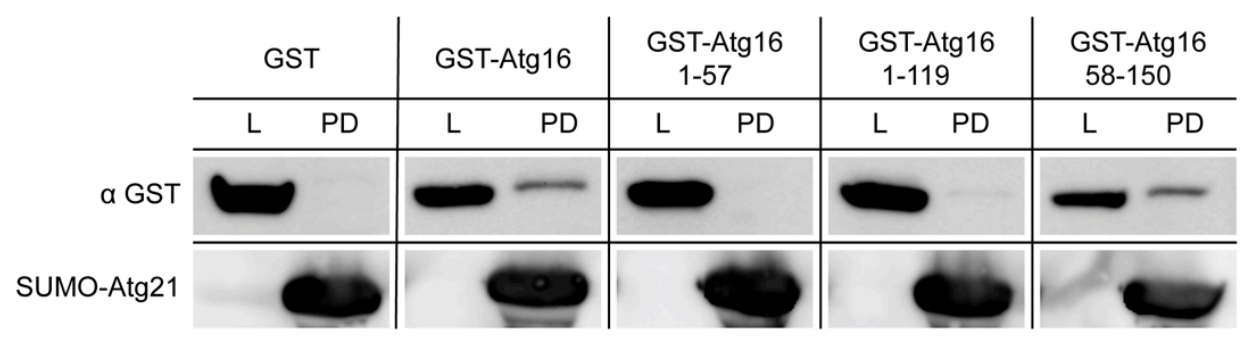

C

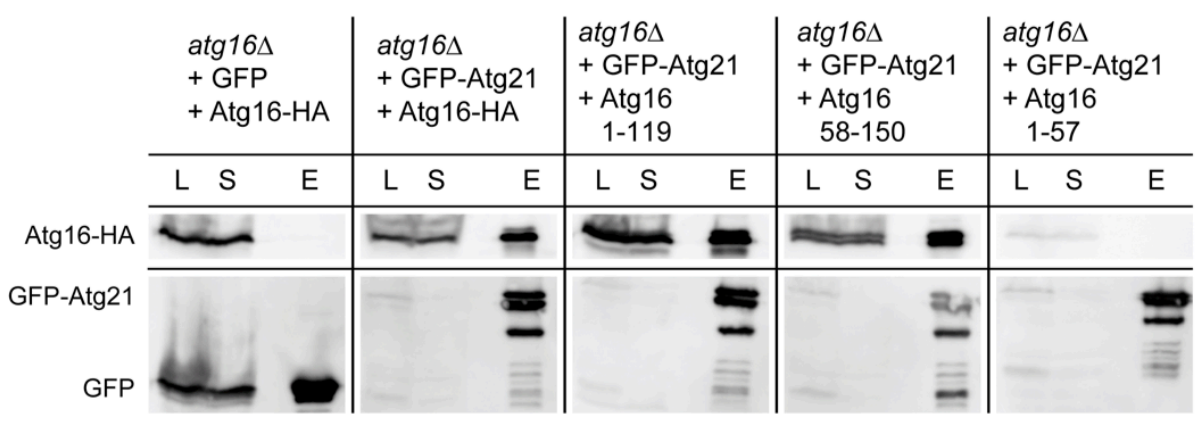

Figure 34: Atg21 interacts with the coiled-coil domain of Atg16

(A) Predicted domain structure of Atg16. Numbers indicate the respective amino acid position. It can not be excluded that the coiled-coil domain might be longer than proposed (Fujioka et al., 2010) (B) Immobilized recombinant 6xHis-SUMO-Atg21 was incubated with purified 
recombinant GST, GST-Atg16 or the indicated truncated versions of GST-Atg16. Samples of the purified GST and GST-Atg16 versions (L) and the eluted proteins (PD) were immunoblotted and analyzed using anti-GST and anti-His antibodies. (C) Growing atg16 cells expressing GFP-Atg21 or GFP alone as negative control and the indicated Atg16-HA version were lysed using the mild cell lysis procedure and subjected to GFP-TRAP ${ }^{\circledR}$ analysis. Expression of Atg161-119-HA and Atg1658-150-HA or Atg161-57-HA was adjusted by addition of $25 \mu \mathrm{M} \mathrm{CuSO}_{4}$ or $50 \mu \mathrm{M} \mathrm{CuSO} \mathrm{Cu}_{4}$ to the selective medium for approximately $12 \mathrm{~h}$, respectively. Samples of the cell lysate (L), the unbound fraction (S) and the purified proteins (E) were analyzed by immunoblotting using antiHA and anti-GFP antibodies.

Atg16-HA was specifically purified with GFP-Atg21 but not with GFP alone. Interestingly, Atg161-119-HA and Atg16 58-150-HA bound specifically to GFP-Atg21. Atg161-57-HA was rather unstable and therefore hardly detectable on the immunoblot. However, even overexposed, no co-precipitated Atg161-57-HA could be detected.

Altogether, these data suggested, that the Atg21-binding region might be positioned within the coiled-coil domain of Atg16. Fujioka et al. (2010) defined highly conserved surface residues within the coiled-coil domain of Atg16 that are required for its autophagic function. They measured ApeI maturation and macroautophagy (ALP assay) of a set of mutants under growing and starvation conditions. Mutation to alanine of the aspartic acid at position 101 (D101) and glutamic acid at position 102 (E102) caused strong defects in both pathways when mutated alone or in concert. Hypothetically, Atg16 and Atg21 might have to interact with each other for proper autophagic function. Loss of the interaction by the mentioned mutations in Atg16 might therefore lead to impaired autophagic activity. To test this hypothesis, Atg16-GFP mutants were constructed, that contained mutations of the aspartic acid at position 101 (D101A) and the glutamic acid at position 102 (E102A) either individual or in combination, and observed in direct fluorescence microscopy. atg8 $8 \mathrm{atg} 16 \Delta$ cells expressing mCherry-Atg21 and the respective Atg16-GFP mutant were grown to log phase in the presence of $0.3 \mathrm{mM}$ methionine to induce endogenous mCherryAtg21 expression and imaged using a DeltaVision Deconvolution microscope (Figure 35). 


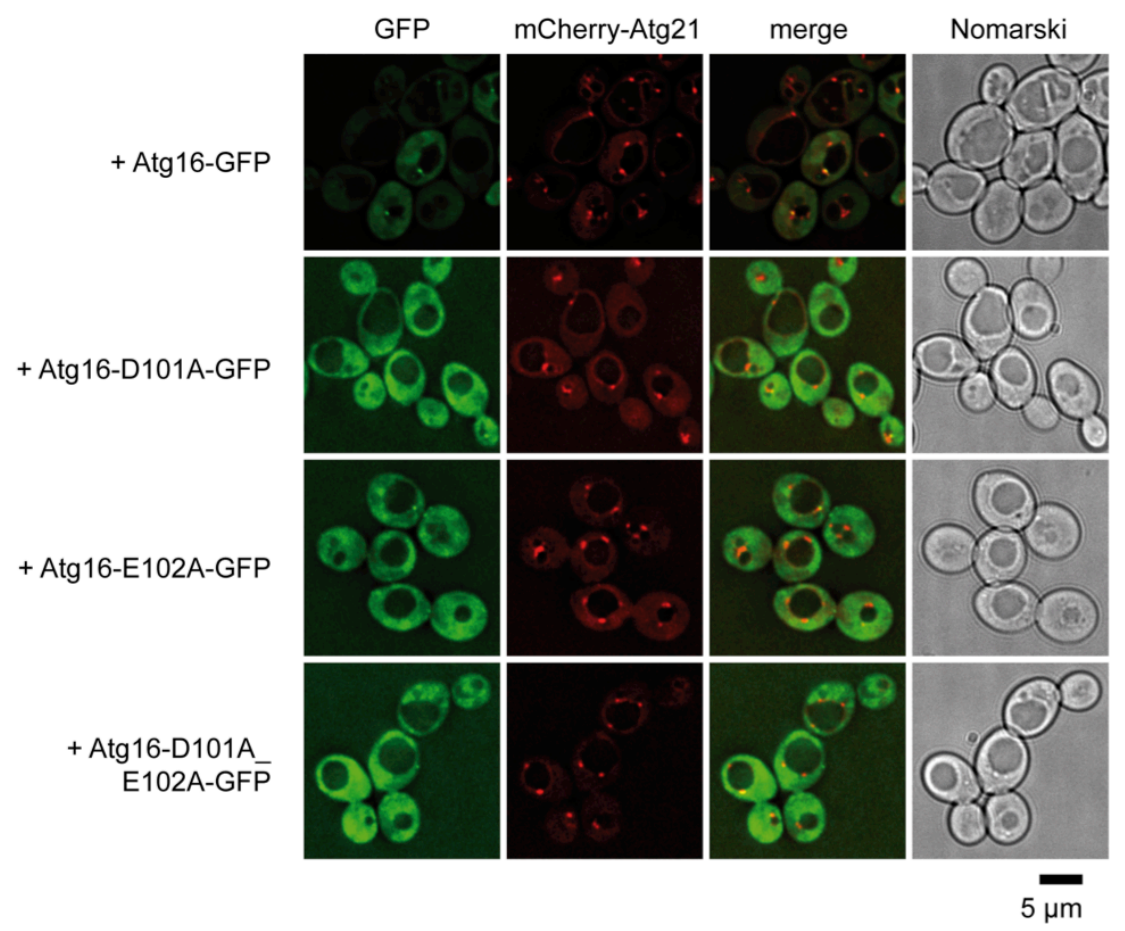

Figure 35: Fluorescence microscopic analysis of the Atg16-GFP point mutants

Growing atg84 atg16 cells expressing mCherry-Atg21 and the indicated Atg16-GFP mutant were grown in the presence of $0.3 \mathrm{mM}$ methionine to log phase and visualized using a DeltaVision Deconvolution microscope equipped with GFP and RFP filter sets. Pictures were deconvoluted using SoftWoRx (Applied Precision) software.

Atg16-GFP formed perivacuolar puncta, which partly colocalized with mCherryAtg21 as described before. In contrast, Atg16 ${ }^{\mathrm{D} 101 \mathrm{~A}}-\mathrm{GFP}, \operatorname{Atg} 16^{\mathrm{E} 102 \mathrm{~A}}-\mathrm{GFP}$ and Atg16 ${ }^{\text {D101A_E102A-GFP }}$ were completely cytosolic. Only Atg16 ${ }^{\mathrm{E} 102 \mathrm{~A}}-\mathrm{GFP}$ rarely formed faint and hardly detectable perivacuolar puncta. The overall perivacuolar punctate appearance of mCherry-Atg21 did not change in the cells expressing the mutant versions of Atg16-GFP.

Next, Atg16-HA mutants were constructed, that contained mutations of the aspartic acid at position 101 (D101A) and the glutamic acid at position 102 (E102A) either individual or in combination. These mutants were subsequently tested for an interaction with Atg21. As a control, Atg16-HA mutants were used, where the lysine at position 95 (K94A) or glutamic acid at position 97 (E97A) were exchanged by alanine, since these mutants exhibited wild type-like autophagic activity (Fujioka et al., 2010). All residues were predicted to have no influence on the formation of the parallel coiled-coil domain conformation. Based 
on the structure, published from Fujioka et al. (2010), the mentioned amino acids are located on the surface of the coiled-coil domain, facing the cytosol (Figure $36 \mathrm{~A}$ ). Nevertheless it was confirmed, that the mutated residues did not change the overall structure of Atg16, which could be another explanation for impaired autophagic function. The coiled-coil domain of Atg16 mediates its dimerization as a prerequisite for the formation of the Atg12-Atg5/Atg16 complex essential for autophagy (Mizushima et al., 1999). In the GFP-TRAP ${ }^{\circledR}$ analysis, Atg16-GFP could only interact and therefore co-precipitate Atg16-HA, if its coiled-coil domain was properly formed. For this purpose, cell lysates of growing atg16 4 cells expressing Atg16-GFP, Atg16 ${ }^{\text {D101A_E102A-GFP, Atg16 }{ }^{\mathrm{K} 94 \mathrm{~A}}-\mathrm{GFP}}$ or GFP alone as control were mixed with the respective cell lysate of growing

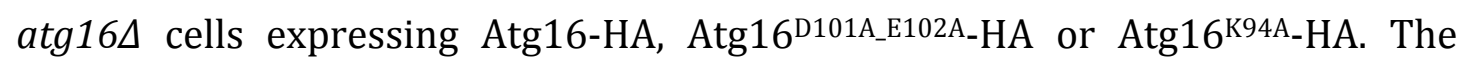
mixture was subjected to GFP-TRAP ${ }^{\circledR}$ analysis and evaluated by immunoblot (Figure 36 B). 
A
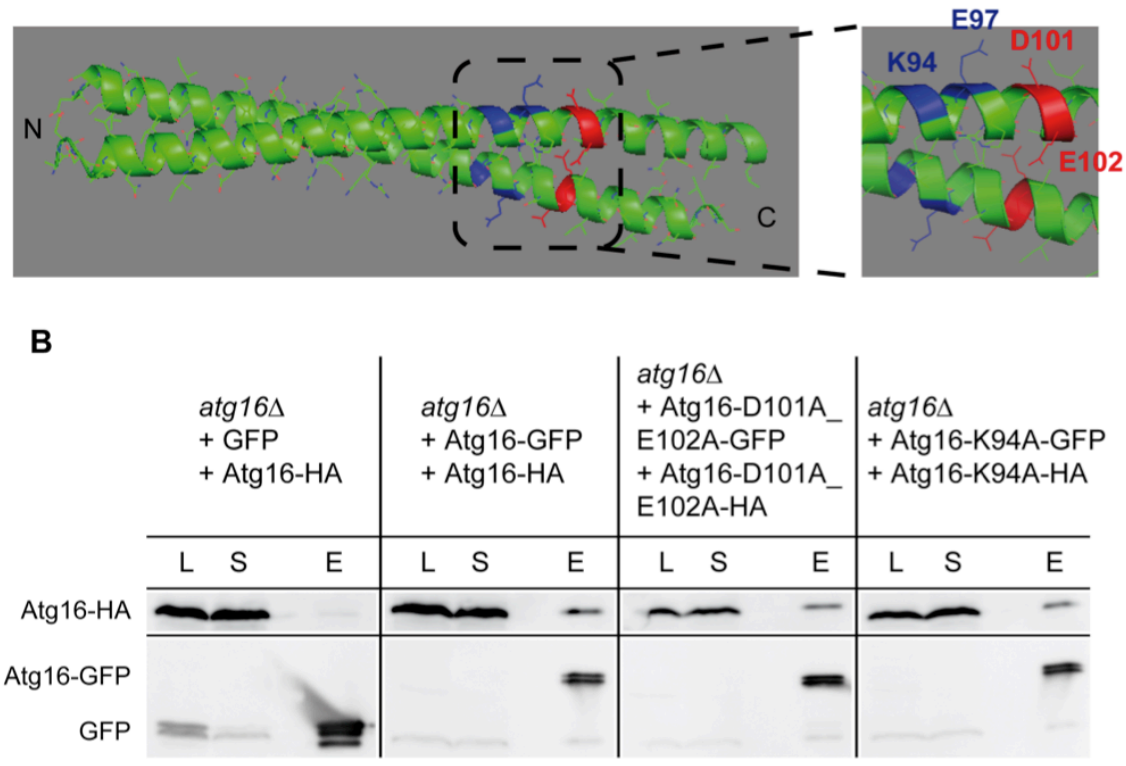

C

\begin{tabular}{|c|c|c|c|c|c|c|c|c|c|c|}
\hline & $\begin{array}{l}\text { atg16 } \\
+ \text { GF } \\
+ \text { Atg }\end{array}$ & & $\begin{array}{l}\operatorname{atg} 16 \Delta \\
+ \text { GFP- } \\
+\operatorname{Atg} 16\end{array}$ & & \begin{tabular}{|}
$\operatorname{atg} 16 \Delta$ \\
+ GFP- \\
+ Atg16 \\
D101
\end{tabular} & & $\begin{array}{l}\operatorname{atg} 16 \Delta \\
+ \text { GFP-A } \\
+ \text { Atg16- } \\
\text { E102A }\end{array}$ & & \begin{tabular}{|l}
$\operatorname{atg} 16 \Delta$ \\
+ GFP-A \\
+ Atg16- \\
E102A
\end{tabular} & $\begin{array}{l}21 \\
111 \mathrm{~A} \\
\mathrm{iA}\end{array}$ \\
\hline & L S & $\mathrm{E}$ & L S & $\mathrm{E}$ & L S S & E & L $\quad S$ & $\mathrm{E}$ & L $\quad S$ & E \\
\hline Atg16-HA & 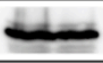 & & L & $=$ & $\sim$ & - & - & - & $=$ & $-x$ \\
\hline P-Atg21 & & & & $=$ & $=$ & $=$ & $=$ & $=$ & $=$ & \\
\hline & & & $-\cdots$ & $\equiv$ & -- & $=$ & -- & $\equiv$ & - & $\Rightarrow$ \\
\hline
\end{tabular}

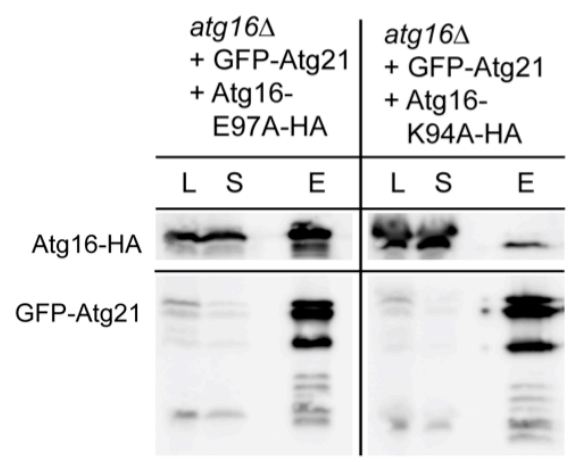

Figure 36: Amino acids D101 and E102 of Atg16 mediate the interaction with Atg21

(A) Ribbon diagram of the dimerized coiled-coil domain of Atg16. The amino acids lysine at position 94 (K94) and glutamic acid at position 97 (E97) are colored blue; aspartic acid at position 101 (D101) and glutamic acid at position 102 (E102) are colored red. The structural model was prepared based on the structural data reported by Fujioka et al. (Fujioka et al., 2010). $\mathrm{N}$ : N-Terminus; C: C-terminus (B) Cell lysate of growing atg16 4 cells expressing GFP as negative control or the indicated Atg16-GFP construct was mixed with cell lysate of growing atg16 cells expressing the indicated Atg16-HA construct. The mixture was subjected to GFP-TRAP ${ }^{\circledR}$ analysis. 
Samples of the cell lysate (L), the unbound fraction (S) and the purified proteins (E) were analyzed by immunoblotting using anti-HA and anti-GFP antibodies. (C) Growing atg16 cells expressing GFP-Atg21 or GFP as negative control and the indicated Atg16-HA version were lysed using the mild cell lysis procedure and subjected to GFP-TRAP ${ }^{\circledR}$ analysis. Evaluation was performed as described in (B).

Atg16-GFP specifically co-precipitated Atg16-HA, whereas GFP alone did not.

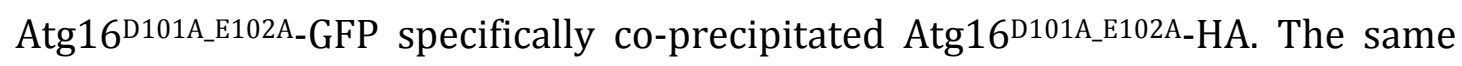
was observed for Atg16 ${ }^{\mathrm{K} 94 \mathrm{~A}}-\mathrm{GFP}$ and Atg16 ${ }^{\mathrm{K} 94 \mathrm{~A}}-\mathrm{HA}$. In conclusion, the formation of their coiled-coil domain was most likely not affected as the GFP-tagged wild type and mutants were all able to co-precipitate their HA-tagged counterparts.

Next, atg16 cells expressing GFP-Atg21 or GFP alone as control and the respective Atg16-HA construct were grown to mid-log phase and lysed in a mild way. The cell lysates were subjected to GFP-TRAP ${ }^{\circledR}$ analysis and evaluated by immunoblotting (Figure $36 \mathrm{C}$ ). Atg16-HA bound specifically to GFP-Atg21, but not to GFP alone. Interestingly, the binding of Atg16 ${ }^{\mathrm{E} 102 \mathrm{~A}}-\mathrm{HA}$ and $\mathrm{Atg} 16^{\mathrm{K} 94 \mathrm{~A}}-\mathrm{HA}$ to GFP-Atg21 was severely impaired, whereas the binding of Atg16 ${ }^{\mathrm{D} 101 \mathrm{~A}}-\mathrm{HA}$ and

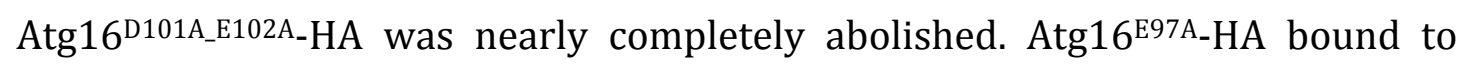
GFP-Atg21 in a wild type-like manner.

Both proteins, Atg21 and Atg16, play a crucial role in the ubiquitin-like conjugation of Atg8 to PE. If the interaction of Atg21 and Atg16 is needed for proper Atg8 lipidation, the Atg8-PE formation should be affected in the Atg21binding defective Atg16 mutants. Therefore, the Atg8-PE formation in the presence of the Atg21-binding defective Atg16 mutants was tested. WT, atg1 , $\operatorname{atg} 3 \Delta, \operatorname{atg} 8 \Delta, \operatorname{atg} 16 \Delta$ and $\operatorname{atg} 21 \Delta$ cells expressing the empty vector were used as controls. atg16 cells expressing Atg16-HA or the indicated either truncated or mutated Atg16-HA version from the CUP1 promoter were grown to mid-log phase. Samples were lysed using a mild glass bead lysis protocol and prepared for SDS-PAGE as described in Suzuki et al. (Suzuki et al., 2001). To adjust the expression level of the Atg16-HA versions, the expression of Atg161-57-HA, Atg161-119-HA and Atg16 58-150-HA was adjusted by the addition of $50 \mu \mathrm{M}, 10 \mu \mathrm{M}$ or $25 \mu \mathrm{M} \mathrm{CuSO}_{4}$ to the selective medium, respectively. The samples were separated on a 15\% SDS-PAGE containing $6 \mathrm{M}$ urea to distinguish between Atg8 
and Atg8-PE. The SDS-PAGE was subsequently immunoblotted and analyzed by several antibodies (Figure 37). As a loading control, the immunoblot was decorated with anti-PGK antibody, confirming similar protein amounts in all samples.

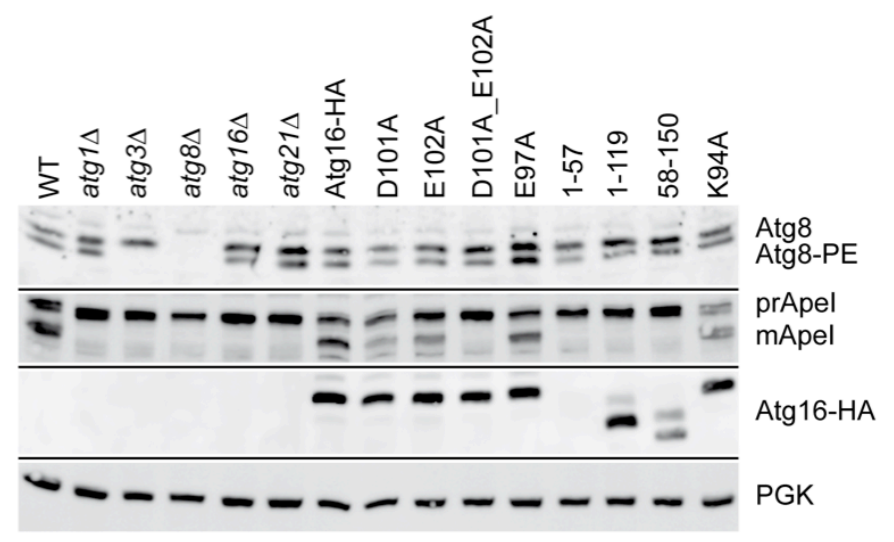

Figure 37: Functional analysis of the Atg16 point mutants

WT, $\operatorname{atg} 1 \Delta, \operatorname{atg} 3 \Delta, \operatorname{atg} 8 \Delta, \operatorname{atg} 16 \Delta, \operatorname{atg} 21 \Delta$ cells carrying the empty vector or $\operatorname{atg} 16 \Delta$ cells expressing the indicated Atg16-HA version were lysed in mid-log phase using a mild glass beads lysis protocol described in Suzuki et al. (2001). The expression of Atg161-57-HA, Atg16 1-119-HA and Atg1658-150-HA was adjusted by the addition of $50 \mu \mathrm{M}, 10 \mu \mathrm{M}$ or $25 \mu \mathrm{M} \mathrm{CuSO}_{4}$ to the selective medium over night $(12 \mathrm{~h})$, respectively. Samples were subjected to SDS-PAGE containing $6 \mathrm{M}$ urea to separate Atg8 and Atg8-PE, immunoblotted and analyzed using anti-Atg8, anti-ApeI, antiHA and anti-PGK antibodies.

In WT and atg1 $\Delta$ cells, where the lipidation of Atg8 is unaffected, approximately $50 \%$ of Atg8 was conjugated to PE. In atg3 $\Delta$ cells, defective in Atg8 lipidation, Atg8 was present solely in its unconjugated form. In $\operatorname{atg} 16 \Delta$ and $\operatorname{atg} 21 \Delta$ cells, the lipidation of Atg8 is severely impaired, so that most of Atg8 was present in its unlipidated form. Atg16-HA completely complemented the atg16 phenotype, as Atg8-PE was formed in a wild type-like manner. The same was observed in atg16 cells expressing Atg16 ${ }^{\mathrm{E} 97 \mathrm{~A}}$-HA. In contrast, Atg8-PE formation was severely impaired in atg16 cells expressing Atg16 ${ }^{\mathrm{D} 101 \mathrm{~A}}-\mathrm{HA}, \mathrm{Atg} 16^{\mathrm{E} 102 \mathrm{~A}}-\mathrm{HA}$,

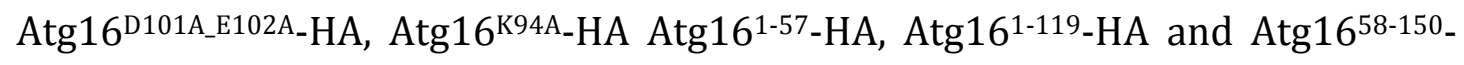
HA comparable to $\operatorname{atg} 16 \Delta$ or $\operatorname{atg} 21 \Delta$ cells. Except of Atg161-57-HA, all Atg16-HA versions were present in comparable amounts.

The immunoblot was in addition decorated with an anti-ApeI antibody to monitor the autophagic activity. In WT cells, about half of ApeI was present in 
the matured form. As expected, the maturation of ApeI was completely blocked in $\operatorname{atg} 1 \Delta, \operatorname{atg} 3 \Delta, \operatorname{atg} 8 \Delta, \operatorname{atg} 16 \Delta$ and $\operatorname{atg} 21 \Delta$ cells. Atg16-HA fully complemented Apel maturation when expressed in atg16 cells. The same was observed for Atg16 ${ }^{\mathrm{E} 97 \mathrm{~A}}-\mathrm{HA}$ and Atg16 ${ }^{\mathrm{K} 94 \mathrm{~A}}-\mathrm{HA}$. Apel maturation was severely impaired in atg16 cells expressing Atg16 ${ }^{\mathrm{D} 101 \mathrm{~A}}-\mathrm{HA}$ and Atg16 ${ }^{\mathrm{E} 102 \mathrm{~A}}-\mathrm{HA}$ mutants. In atg16 cells expressing Atg16 D101A_E102A-HA, Atg161-57-HA, Atg161-119-HA or Atg1658-150_ HA the maturation of ApeI was completely blocked.

In conclusion, Atg16 interacted directly with Atg21 most likely via its coiled-coil domain. The conserved surface residues, E102 and more important D101, were identified to mediate proper interaction of both proteins. Atg21 and Atg16 interaction was a prerequisite for proper Atg8 lipidation and autophagic function. 


\section{Discussion}

Autophagy is an important cellular degradation and recycling process. It is characterized by the formation of double membrane layered transport vesicles, the autophagosomes. In yeast, the formation of these autophagosomes starts at the pre-autophagosomal structure (PAS) as a cup shaped isolation membrane (phagophore) that expands and thereby encloses cytoplasm and organelles. The autophagosome finally fuses with the vacuolar membrane to release a still one membrane layered vesicle (autophagic body) into the vacuole lumen, where it is lysed. The contents are degraded and finally recycled. There are also several selective types of autophagy like the Cvt pathway in yeast. During this pathway the vacuole hydrolases aminopeptidase I, $\alpha$-mannosidase and aspartyl aminopeptidase are constitutively transported from the cytoplasm to the vacuole (see chapter 2.2).

In this study, the localization and function of Atg21, one of the proteins essentially required for the Cvt pathway, were investigated using the model organism Saccharomyces cerevisiae. Autophagy is highly conserved among all eukaryotes. Therefore, most of the autophagy-related proteins are conserved from yeast to mammals. This conservation includes their function and recruitment hierarchy to the autophagosome formation site (Itakura and Mizushima, 2010). Thus, information obtained in yeast can often be transferred to higher eukaryotes.

Atg21 and its yeast homologues Atg18 and Hsv2 are members of the family of $\beta$ propellers that bind polyphosphoinositides (PROPPINs). They are WD40 repeat propellers and contain two lipid binding pockets, that preferential bind to PI3P and PI(3.5) $\mathrm{P}_{2}$ (Baskaran et al., 2012; Krick et al., 2012; Watanabe et al., 2012). Atg21 and its homologues are highly conserved, but required for different variants of autophagy. While macroautophagy is only significantly reduced in $\operatorname{atg} 21 \Delta$ cells, Atg21 is required for selective types of autophagy like the Cvt pathway (Barth et al., 2002). Selective types of autophagy require the very same core autophagy proteins involved in bulk autophagy. Therefore, information 
obtained in the selective variants can often be transferred to other types of autophagy.

Mammals contain four PROPPIN orthologs, termed WIPIs. Based on phylogenetic tree analysis, WIPI-1 and WIPI-2 were assigned to Atg18 and WIPI-3 and WIPI-4 to Hsv2 (Krick et al., 2012; Polson et al., 2010), whereas Atg21 orthologs have been only found in yeast species so far (Meijer et al., 2007). However, WIPI-1 seems to be involved in LC3 lipidation (Polson et al., 2010; Proikas-Cezanne et al., 2004). In addition, WIPI-2 was reported to positively regulate the lipidation of the mammalian Atg8 ortholog LC3 (Polson et al., 2010). A similar function has been observed specifically for yeast Atg21 (Meiling-Wesse et al., 2004; Stromhaug et al., 2004), suggesting that not the protein itself, but its elementary function in the autophagic process might be conserved in higher eukaryotes.

In addition to the efficient lipidation of Atg8, Atg21 is required for recruitment of Atg8 and Atg5 to the PAS (Meiling-Wesse et al., 2004; Stromhaug et al., 2004). Therefore, no Cvt vesicles are formed in the absence of Atg21, suggesting an elementary role for Atg21 in the PAS assembly especially in selective types of autophagy. Thus, Atg21 has not been detected at the PAS so far.

\subsection{Part of Atg21 localizes to the PAS}

In this study, a potential PAS localization of Atg21 was investigated using direct fluorescence microscopy. Therefore, atg21 cells expressing Atg21-YFP from its endogenous promoter and the PAS marker ApeI-RFP were imaged. Cells were analyzed under growing conditions, as Atg21 is strictly required for the constitutive Cvt pathway acting under those conditions.

Usually, Atg8 is used as a PAS marker in Atg protein localization studies. In this study, ApeI-RFP was used as a PAS marker for two reasons. First, the expression of Atg8 is upregulated at autophagy-inducing conditions, but under growing conditions the amount of Atg8 is significantly smaller (Huang et al., 2000). Therefore, the part of Atg8 at the PAS is hardly detectable in fluorescence microscopy and not suitable for PAS rate determination under these conditions. Second, in this study Atg8 was identified as a potential interaction partner of Atg21 (see chapter 5.6). Therefore, Atg21 and Atg8 could form a complex in the cytosol that is subsequently recruited to the PAS. This has been reported for the 
Atg21 homologue Atg18 and its interaction partner Atg2 (Obara et al., 2008b). A cytosolic Atg21-Atg8 complex would mimick a colocalization at the PAS. Therefore, the PAS marker ApeI-RFP was used as an alternative. In addition, ApeI is selectively transported to the PAS via the Cvt pathway, where Atg21 is active.

The percentage of ApeI-RFP dots that colocalized with Atg21-YFP was determined (Colocalization rate). In fact, a considerable number of ApeI-RFP respectively PAS dots (49\%) colocalized with Atg21-YFP, indicating that indeed a part of Atg21 is present at the PAS (see Figure 11). That is the first time that a part of Atg21 has been detected at the PAS.

Atg19 is the Cvt cargo receptor and directs the ApeI complex to the PAS by binding to Atg11 and Atg8 (Kim and Klionsky, 2000; Shintani et al., 2002). The colocalization rate in $\operatorname{atg} 19 \Delta$ cells was determined to exclude an interaction of Atg21 and ApeI. As expected, the colocalization rate of Atg21-YFP and perivacuolar ApeI-RFP dots was significantly reduced in atg194 cells (16\%) compared to the wild type (atg21 $=49 \%)$. Hence, ApeI-RFP is a suitable PAS marker for colocalization studies with Atg21-YFP under the selected conditions. Atg11 is a component of the Atg1 kinase complex and acts as a scaffold for PAS assembly for selective types of autophagy. In addition, it acts in cargo recognition via direct binding of receptor proteins like Atg19 in the Cvt pathway. Consequently, the Cvt complex is not directed to the PAS, although Atg19 can still bind to the ApeI complex (Shintani et al., 2002). Indeed, the colocalization rate of Atg21-YFP and ApeI-RFP was significantly reduced in the absence of Atg11 (31\%). Unexpectedly, the colocalization rate was only slightly reduced compared to atg194 cells (16\%), though both deletions should result in the absence of ApeI-RFP from the PAS. Atg11 might be partially replaced by Atg17 that adapts the Atg11 scaffold function in the Atg1 kinase complex under autophagyinducing conditions (Cheong et al., 2008; Suzuki and Ohsumi, 2007; Suzuki et al., 2007). However, it is not known, if all proteins are simultaneous present in the Atg1 kinase complex or if there are subcomplexes, that vary dependent on nutrient conditions. The amount of Atg11, Atg17 and Atg19 at the PAS are proposed to be the same under both growing and starvation conditions (Geng et al., 2008). Furthermore, though bulk autophagy is dramatically induced under 
stress conditions, it occurs also at a basal level under normal growth conditions (Parzych and Klionsky, 2013; Yorimitsu and Klionsky, 2005). Therefore, atg11 $\operatorname{atg} 17 \Delta$ double mutants are usually used to completely block autophagosome formation. Speculatively, the lack of Atg11 could be partially complemented by Atg17 even under nutrient-rich conditions. This would result in a PAS formation at a low frequency and Atg19 would still be able to direct the ApeI complex to the PAS by binding to Atg8. In contrast, in the absence of the receptor Atg19, the ApeI complex is not able to bind to any PAS component. This could explain, why the deletion of ATG19 has a more severe effect than the deletion of ATG11. To prove this hypothesis, the colocalization rate of Atg21-YFP and ApeI-RFP should be further determined in $\operatorname{atg} 11 \Delta \operatorname{atg} 17 \Delta$ cells.

Atg21 is peripherally membrane associated by binding to $\mathrm{PI} 3 \mathrm{P}$ and/or $\mathrm{PI}(3,5) \mathrm{P}_{2}$ (Krick et al., 2006). Yeast has only one phosphoinositide 3-kinase, Vps34, which is present in two complexes. Each complex contains in addition to the core components a unique factor, that links the kinase complex to a specific site and therefore to a specific cellular function. Vps38, the unique subunit of complex II, recruits the kinase complex to endosomes (Kihara et al., 2001; Obara et al., 2006). Atg21-YFP formed one to six perivacuolar dots within the cell. Some of those have been colocalized earlier with endosomal marker proteins as FYVERFP or Snf7-RFP (Krick et al., 2008a). The localization of Atg21 at endosomal compartments is dependent on Vps38 and therefore on the endosome-specific PI3P pool (Krick et al., 2008a). The autophagy-specific Vps34 complex I at the PAS specifically contains Atg38 and Atg14 (Araki et al., 2013; Obara et al., 2006). In the absence of Atg14 respectively the PAS-specific PI3P pool, Atg21 should be also absent from the PAS. Indeed, the colocalization rate of Atg21-YFP and ApeIRFP was significantly reduced in atg14A cells (22\%) compared to wild type cells (49\%), confirming that the PAS localization of Atg21 is PI3P-dependent. In contrast to vps384 cells, where Atg21 seems to be nearly completely released to the cytosol (Krick et al., 2008a), the deletion of ATG14 did not lead to a change in the overall punctate appearance of Atg21-YFP. Under growing conditions, the majority of PI3P have been reported to locate at endosomes and the vacuolar membrane, whereas only a minor amount has been found at the PAS (Obara et al., 2008a). Therefore, probably only a small part of Atg21-YFP is located at the 
PAS, whereas the major part is located at other sites as endosomes. That might also be a reason why Atg21 has not been detected at the PAS before. Stromhaug et al. (2004) have explicitly monitored cells expressing chromosomally tagged Atg21-GFP and prApeI-RFP as PAS marker and observed, that these two proteins did not colocalize. The endogenous expression level of Atg21 is quite low leading to a weak fluorescence signal. The Atg21-YFP construct used in this study was also expressed from its endogenous promoter, but a higher resolution microscopy has been used for analysis. Therefore, that Atg21 has not been detected at the PAS before could be due to a difference in the microscope sensitivity. Furthermore, the PAS localization of Atg21 was not immediately obvious. Though the Cvt pathway is highly active under the elected conditions, only $35 \%$ of the cells averaged a perivacuolar ApeI-RFP respectively PAS dot. In addition, Atg21-YFP forms approximately three to six dots within the cell. For the quantification of the Atg21 PAS localization, it was necessary to determine the ApeI-RFP and Atg21-YFP dots in a high number of cells to validate the results.

As shown for the Atg21 homologue Atg18, proper membrane association is mediated by two components, the PIP binding and an additional protein interaction partner like Atg2 (Obara et al., 2008b; Rieter et al., 2013). The PAS localization of the Atg18-Atg2 complex additionally requires the membrane protein Atg9 (Reggiori et al., 2004). Atg9 forms a trimeric complex with Atg23 and Atg27. All three proteins cycle between the PAS and a non-PAS pool and the PAS recruitment of all three proteins depends on the presence of each other. In contrast, the retrograde transport of Atg9 from the PAS to its non-PAS pool is independent of Atg23 and Atg27 but dependent on the Atg2-Atg18 complex (Legakis et al., 2007). It has been already shown by two independent approaches that the PIP binding is required for proper Atg21 localization. First, Atg21 is not present at the PAS in the absence of PI3P (see above). Second, mutations within the lipid binding domain of Atg21 result into release of the protein into the cytosol (Krick et al., 2006). In order to find the additional protein component required for proper PAS localization of Atg21, further deletion strains were tested for a loss of the Atg21 PAS localization (see Figure 11+Figure 12). 
In the absence of Atg9, the PAS localization of Atg21-YFP was significantly reduced (26\%), pointing to a potential influence of Atg9 on the Atg21 PAS localization. In contrast, further split ubiquitin approaches did not detect an interaction of $\operatorname{Atg} 21$ and Atg9 (see chapter 5.5). Thus, the effect of Atg9 is probably indirect, because Atg9 is required for the recruitment of Atg14 to the PAS and therefore for the generation of the PAS-specific PI3P pool (Suzuki et al., 2007). The absence of Atg2 or Atg27 had only a slight effect on the PAS localization of Atg21-YFP (34\% respectively 38\%), whereas the deletion of ATG23 did not change the colocalization rate of Atg21-YFP (48\%). The Atg2Atg18 complex is required for efficient retrograde transport of Atg9 from the PAS (Reggiori et al., 2004). Therefore, the loss of Atg2 could have a rather indirect effect on the Atg21 PAS localization. Similarly, also Atg27 and Atg23 are required for the transport of Atg9 to the PAS (Legakis et al., 2007). Nonetheless, the loss of Atg27 but not of Atg23 slightly affected the Atg21 PAS localization. In addition, split-ubiquitin approaches detected a potential interaction of Atg27 and Atg21 (see Figure 21), pointing to a stabilizing role for Atg27 in Atg21 PAS localization. However, a potential interaction of Atg21 and Atg27 was analyzed in further experiments and are discussed in more detail below (see chapter 5.5). As mentioned before, Atg21 is required for the recruitment of Atg8 and Atg5 to the PAS (Meiling-Wesse et al., 2004; Stromhaug et al., 2004). Atg8 is conjugated to PE in a ubiquitin-like manner, where the Atg12-Atg5/Atg16 complex functions as an E3-like enzyme ((Nakatogawa, 2013), see chapter 2.2.4.4). Both, Atg8 and Atg12-Atg5/Atg16 are among the last components, that are recruited to the PAS (Suzuki et al., 2007). Furthermore, Atg16 mediates the PAS localization of the Atg12-Atg5/Atg16 complex (Hanada et al., 2007; Suzuki et al., 2001). The loss of Atg8 did not affect the PAS localization of Atg21-YFP, whereas the Atg21 PAS localization was slightly reduced in the absence of Atg16 (Colocalization rate: 52\% respectively 34\%). Therefore, the Atg21 PAS localization is most likely independent on Atg8, but stabilized by Atg16. As discussed below, this study revealed that Atg21 and Atg16 interact directly with each other. Furthermore, two surface residues within the coiled-coil domain were identified to specifically bind to Atg21 (see chapter 4.3.6.4). The Atg12-Atg5/Atg16 complex is proposed to be dimeric mediated by the coiled-coil domain of Atg16 (Fujioka et al., 2010; 
Kuma et al., 2002). Therefore, one Atg12-Atg5/Atg16 complex exhibits two Atg16 molecules and thus two potential Atg21 binding sites. It could be possible, that Atg16 bridges two Atg21 molecules resulting in a dimeric Atg21-Atg16 respectively Atg21-Atg16/Atg5-Atg12 complex. The Atg21 dimerization would further stabilize the PI3P-dependent membrane association of Atg21. Consequently, the Atg21 PAS localization could be destabilized in the absence of Atg16 as observed in this study. This hypothesis is further supported by the observation, that Atg5 is not required for the colocalization of Atg16-GFP and mCherry-Atg21 at the PAS (see chapter 4.3.6.2 and Figure 32). Dimerization of Atg16 is independent of Atg5 and Atg12 (Mizushima et al., 1999). Thus, Atg16 alone would be sufficient to bridge Atg21 and thus stabilize the membrane association of Atg21 at the PAS. This model would also not exclude a potential additional interaction with the membrane protein Atg27 to stabilize membrane association. As mentioned above, the Atg21 homologue Atg18 binds to the cytosolic protein Atg2, and both proteins are recruited to the PAS by additional interaction with the membrane protein Atg9 (Reggiori et al., 2004). Similarly, interaction with the membrane protein Atg27 in addition to the cytosolic Atg16 could enhance the Atg21 PAS localization. However, this model has to be proven by further Atg21 localization studies. The PAS localization of Atg21 should be determined in $\operatorname{atg} 5 \Delta$ and $\operatorname{atg} 12 \Delta$ cells to exclude an influence of the remaining components of the Atg12-Atg5/Atg16 complex on the Atg21 PAS localization. Importantly, the deletion of Atg16 or Atg27 had only a slight effect on the Atg21 PAS localization, suggesting that more than one component might stabilize the Atg21 PAS localization. This implies that the simultaneous deletion of two or more components should result in a more severe defect in Atg21 PAS localization. Therefore, the determination of the Atg21 PAS localization in $\operatorname{atg} 14 \Delta \operatorname{atg} 16 \Delta, \operatorname{atg} 14 \Delta \operatorname{atg} 27 \Delta$ or atg16 $\operatorname{atg} 27 \Delta$ cells should be further determined to prove the hypothesis.

In conclusion, part of Atg21 clearly localizes at the PAS in a PI3P-dependent manner. As mentioned before, the assembly of the PAS and the recruitment of the Atg proteins is a quite dynamic process and occurs in a specific hierarchy. Based on the results obtained from the PAS localization analysis of Atg21-YFP in this study, Atg21 is most likely recruited to the PAS after localization of the 
Atg14-containing Vps34 kinase complex and therefore after the generation of the PAS-specific PI3P pool, but before recruitment of Atg8 and its E3-like enzyme, the Atg12-Atg5/Atg16 complex.

\subsection{Atg21 is located on the outer autophagosomal membrane}

Atg21 is peripherally membrane associated at the PAS via binding of PI3P (see Figure 11). In the current model, the PAS assembles through hierarchical interactions between Atg proteins and generates the phagophore, which expands to become an autophagosome. The specific localization of each protein at the PAS seems to correspond directly to its respective function (Suzuki et al., 2013).

A more detailed insight into the Atg protein distribution at the PAS and across the phagophore membrane, was reported by Suzuki et al. (2013). By high overexpression of prApeI, a giant prApeI complex is generated, that is too large to be packed into a Cvt vesicle or even in an autophagosome. Instead, the expansion of the phagophore arrests at its maximum length, resulting in a cupshaped like structure associated with both the giant prApeI complex and the vacuolar membrane. Moreover, the distribution pattern of the Atg proteins across the phagophore and the PAS is also arrested and can be visualized by direct fluorescence microscopy. Unfortunately, the distribution of Atg21 was not monitored, probably because it has not been detected at the PAS so far. However, the Atg21 homologue Atg18 and its interaction partner Atg2 localize to the edges of the expanding phagophore (Suzuki et al., 2013). As discussed before, in contrast to Atg18, Atg2 most likely only indirectly affects the PAS localization of Atg21. Therefore, the localization pattern of Atg18 can probably not be transferred to Atg21.

Atg8 is distributed evenly across the outer and inner membrane of the expanding phagophore, whereas the Atg12-Atg5/Atg16 complex is restricted to the outer membrane (Kirisako et al., 2000; Mizushima et al., 2001; Suzuki et al., 2001; Suzuki et al., 2013). As discussed below in more detail, Atg21 probably interacts with Atg8 and the Atg12-Atg5/Atg16 complex (see chapter 5.6+5.7). At least the interaction with the Atg12-Atg5/Atg16 complex occurs at the PAS. Furthermore, Atg21 probably mediates membrane association of the Atg12Atg5/Atg16 complex by direct binding of Atg16 (see chapter 5.7), suggesting that 
Atg21 and the Atg12-Atg5/Atg16 complex share the same localization pattern on the forming autophagosome. In contrast, PI3P that mediates the peripheral membrane association of Atg21, is distributed across the inner and outer autophagosomal membrane (Obara and Ohsumi, 2008). But among the autophagy-related proteins only those on the inner membrane of the expanding phagophore are trapped inside the matured autophagosome and transported into the vacuole. Using direct fluorescence microscopy, a potential transport of

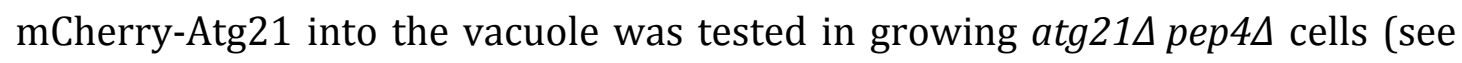
Figure 14). mCherry-Atg21 is most probably not transported into the vacuole. Therefore, as the Atg12-Atg5/Atg16 complex, Atg21 is most likely restricted to the outer membrane of the expanding phagophore and dissociates from that structure immediately before or after completion of the autophagosome as it was suggested for the Atg12-Atg5 complex before (Suzuki et al., 2001). Nevertheless, using the above-mentioned giant ApeI complex approach, the localization of Atg21 across the autophagosomal membranes should be determined in further experiments. Electron microscopy could be additionally used to prove a potential restriction of Atg21 to the outer autophagosomal membrane. Up to now, a transport of the Atg21 homologue Atg18 into the vacuole has also not been reported, probably because Atg18 is not located across the expanding membranes but at the edges of the phagophore (Suzuki et al., 2013).

Maturation and/or fusion of the autophagosomes with the vacuolar membrane require the dissociation of the autophagic machinery. The covalent anchored Atg8 is cleaved off by Atg4 (Kirisako et al., 2000), whereas the major part of the autophagic machinery is probably released by the degradation of PI3P via the phosphatase Ymr1 (Cebollero et al., 2012). As shown for Atg18, proper membrane association is mediated by two components, the PIP binding and an additional protein interaction partner. The degradation of PI3P leads to its dissociation from the membrane (Cebollero et al., 2012). If this was also true for Atg21, Atg21 should remain associated with autophagosomes in the absence of Ymr1 as shown for its homologue Atg18. Since those autophagosomes are not able to fuse with the vacuole (Cebollero et al., 2012), the deletion of YMR1 would result in an increased colocalization of Atg21-YFP and the Cvt vesicle marker ApeI-RFP in fluorescence microscopy. Therefore, the influence of Ymr1 
respectively clearance of PI3P on the dissociation of Atg21 from the autophagosome should be further tested by determination of the colocalization rate of Atg21-YFP and ApeI-RFP as described before (see chapter 4.2.1).

Phosphorylation of the PIP binding site of Atg18 from Pichia pastoris regulates its ability to bind to $\mathrm{PI}(3,5) \mathrm{P}_{2}$ at the vacuolar membrane (Tamura et al., 2013), pointing to an additional regulatory mechanism for PROPPIN dissociation. Along this line, the serine/threonine kinase activity of Atg1 is suggested to specifically function in the regulation of Atg protein localization at the PAS (Cheong et al., 2008). Atg21 has originally been identified as a target of the Atg1 kinase (Ptacek et al., 2005). That led to the idea, that Atg1 could regulate the Atg21 PAS localization via phosphorylation. Nevertheless, a potential influence of Atg1 on the PAS localization of Atg21 is highly speculative and has to be carefully analyzed further.

The respective interaction partner could represent another regulatory level for membrane dissociation. Recently, a potential posttranslational modification site was identified within Atg18, which could contribute to the regulation of the Atg18-Atg2 interaction or other functions of Atg18 (Rieter et al., 2013). Therefore, identification of the interaction partner of Atg21 at the PAS or endosomes could give more hints for a regulation of the Atg21 localization within the cell.

In contrast to Atg21 and Atg18, two of the mammalian PROPPIN orthologs, WIPI-1 and WIPI-2 are located on the inner and outer membrane of generated autophagosomes (Proikas-Cezanne et al., 2011), suggesting a different sorting mechanism in mammals and/or a function different from their yeast counterparts.

\subsection{Atg21 is part of a high molecular weight complex}

Atg21 was detected as a part of a high molecular weight complex of $\geq 670 \mathrm{kDa}$ using gelfiltration chromatography and density gradient centrifugation (see Figure 16+Figure 18). This high molecular weight Atg21 complex was highly sensitive to the cell lysis and buffer conditions (see chapter 4.3.1). In this study, a mild cell lysis procedure was established that keeps the sensible complex intact 
as a prerequisite not only for further analysis of the complex but also for the identification and verification of potential Atg21 interacting proteins.

Sorbitol density gradient centrifugation was used to further analyze the high molecular weight Atg21 complex in depicted mutant strains (see chapter 4.3.1.2). Atg21 is required for the Cvt pathway, which describes the selective transport of ApeI to the vacuole. ApeI forms large dodecamers. One dodecamer has a high molecular weight ( $\sim 670 \mathrm{kDa})$ and was therefore used as a size marker in gelfiltration chromatography and density gradient centrifugation. Otherwise, it would be also possible that the ApeI complex is part of the high molecular weight Atg21 complex. That would also explain the co-elution respectively -sedimentation in gelfiltration respectively sorbitol gradient centrifugation. However, neither the absence of the cargo receptor Atg19 (see Figure 18) nor the absence of ApeI itself (data not shown) did influence the assembly of the high molecular weight Atg21 complex in this study, suggesting that ApeI is probably not part of that complex.

The type I membrane protein Atg27 is, similar to Atg21, required for the Cvt pathway, but only affects macroautophagy (Yen and Klionsky, 2007). Therefore, Atg27 would be a good candidate for an interaction partner of Atg21. However, the deletion of ATG27 did not influence the assembly of the high molecular weight Atg21 complex. Furthermore, the loss of the kinase Atg1 did also not affect the assembly of the Atg21 complex (see Figure 18). However, it cannot be excluded, that one of the tested proteins is a component of the Atg21 complex anyway. Due to their low molecular weight, a change in the distribution pattern of the Atg21 complex within the sorbitol density gradient centrifugation would only be visible if one of the tested proteins was a stoichiometric component of the complex. It can only be concluded, that none of the tested proteins had an effect on the formation of the high molecular weight Atg21 complex.

Hypothetically, the high molecular weight Atg21 complex could constitute an assembly of Atg18 family members in a large scaffold structure. This hypothesis is supported by the finding that Atg21 interacts with itself and its homologues Atg18 and Hsv2 (split-ubiquitin assay data not shown and (Nair et al., 2010)). As discussed below, 6xHis-SUMO-Atg21 specifically co-precipitated all members of the TOR complex 1 (TORC1) in pull down experiments (see chapter 5.4). 
TORC1 is a huge dimeric complex with a predicted molecular size of $\sim 2 \mathrm{MDa}$, estimated by gelfiltration chromatography (Loewith and Hall, 2011). The high molecular weight Atg21 complex was partly present in fractions corresponding to a much higher molecular weight as the ApeI complex $(670 \mathrm{kDa})$ (see Figure 16+Figure 18). Therefore, TORC1 or part of it could represent another candidate for the high molecular weight Atg21 complex. However, this is highly speculative. For determination of the Atg21 complex components, the amount of the complex should be scaled up to identify the respective proteins by mass spectrometry. The formation of the complex was independent of Atg1 (see Figure 18). In general, the loss of Atg1 results in an arrest of most of the autophagy-related proteins at the PAS. If the Atg21 complex was formed or at least partially present at the PAS, atg1 $1 \Delta$ cells could be used to enrich the amount of the Atg21 complex. However, identification of its components would contribute to elucidate the molecular function of Atg21.

\subsection{Identification of Atg21 interaction partner}

The pull down approach was used to further identify Atg21 interaction partner. Recombinant 6xHis-SUMO-Atg21 and 6xHis-SUMO alone as negative control were purified and incubated with yeast crude cell extract. The co-precipitated proteins were analyzed by both immunoblotting and mass spectrometry (see Figure 19). Curiously, no Atg protein has been identified in mass spectrometry, although mCherry-Atg19 was clearly detected by the anti-Red antibody in immunoblotting. The samples tested by immunoblot were the same that were subsequently subjected to mass spectrometry analysis, excluding differences in the pull down procedure. Based on the immunoblot signal, the amount of mCherry-Atg19 should be above the critical detection level for mass spectrometry. Furthermore, prediction programs did not propose any secondary modifications that would complicate an identification of Atg19 in mass spectrometry analysis. For the mass spectrometry analysis, the samples were first separated by SDS-PAGE. After Coomassie staining, the gel was cut into slices and the protein content of each slice was determined. mCherry-Atg19 has a molecular weight of about $75 \mathrm{kDa}$ corresponding approximately to the molecular weight of 6xHis-SUMO-Atg21 (70 kDa). Therefore, mCherry-Atg19 was probably 
present in the same slice as at least part of 6xHis-SUMO-Atg21. The amount of 6xHis-SUMO-Atg21 was much higher than the amount of mCherry-Atg19 (see Figure 19). This could have resulted in a masking and therefore non-detection of mCherry-Atg19 in mass spectrometry analysis. Along this line, the huge amount of 6xHis-SUMO-Atg21 probably also shifted adjacent proteins when loaded on SDS-PAGE, explaining the slight molecular shift of mCherry-Atg19 in the bound fraction in immunoblotting (see Figure 19).

Surprisingly, all components of the target of rapamycin complex I (TORC1) were specifically co-precipitated by 6xHis-SUMO-Atg21 (see Figure 19). TORC1 consists of Tco89, Kog1, Lst8 and the serine/threonine kinase Tor1 or Tor2 and is most likely dimeric (Loewith and Hall, 2011). Its large size ( $2 \mathrm{MDa}$ ) would make it a good candidate as component of the high molecular weight Atg21 complex detected in gelfiltration chromatography and density gradient centrifugation (see chapter 5.3). TORC1 has a central role in several cellular processes that regulate cell growth in response to nutrients and growth factors. In general, TORC1 is active under growing conditions and is regulated by the availability of nutrients such as nitrogen and carbon (Loewith and Hall, 2011). TORC1 also acts as a negative regulator of autophagy. In yeast, it directly phosphorylates Atg13 (Kamada et al., 2010), which most likely regulates Atg1kinase activity. Under autophagy-inducing conditions, Atg13 is dephosphorylated and autophagy is induced. TORC1 is highly conserved in mammals. Recent studies revealed, that the Atg18 ortholog WIPI-1 specifically regulates TORC1 signaling in melanosome formation by an unknown mechanism, that is distinct from the regulation of autophagosome formation (Ho et al., 2011).

However, at this point, it is highly speculative that Atg21 might regulate TORC1 activity as it was proposed for the PROPPIN ortholog WIPI-1 or that Atg21 is conversely regulated via phosphorylation by TORC1 as Atg13. Further characterization of a potential interaction should clarify, if Atg21 interacts directly with one of the TORC1 components or if Atg21 and TORC1 are simultaneously present in the same complex. Thereby, a potential functional role of this interaction could be proposed. 


\subsection{Interaction studies of Atg21 and Atg27}

The membrane protein Atg27 forms a trimeric complex with the second membrane protein Atg9 and the cytosolic protein Atg23 with Atg9 bridging both. All three proteins cycle between the PAS and a peripheral site. The cycling of each protein to the PAS is dependent on the presence of the other complex components (Legakis et al., 2007). Recent studies revealed that Atg9 resides on peripheral vesicular structures, which directly contributes to the formation of the phagophore upon autophagy induction (Kakuta et al., 2012; Mari et al., 2010; Yamamoto et al., 2012). At least Atg27 is present on the Atg9-containing vesicles, suggesting that the trimeric complex is required for membrane delivery to the PAS (Kakuta et al., 2012). Atg9 is required for this process under both growing and starvation conditions, whereas Atg27 is only required for efficient formation of Cvt vesicles. Therefore, Atg27 has the same autophagy phenotype as Atg21 and could be an appropriate protein interaction partner of Atg21. Indeed, an interaction of both proteins was detected in the split-ubiquitin assay (see Figure 21). Furthermore, Atg21 specifically interacted with Atg27 but not with Atg9 or Atg23. Atg27 contains 271 amino acids and is a type I membrane protein. Therefore, it has one transmembrane domain (residues 199-221) and its Cterminus faces the cytosol, whereas the N-terminus extends into the lumen (Yen et al., 2007) (see Figure 22). Nui-Atg27, where the Nui part was facing the lumen, did not interact with Atg21-Cub. Atg27 contains a N-terminal signal sequence, which is cleaved off after membrane insertion (Yen et al., 2007). In the Nui-Atg27 construct, the Nui part is N-terminally fused to Atg27. This could either result in a masking of the signal sequence and therefore in a mislocalization of Nui-Atg27 or in a cleavage of the Nui part together with the signal sequence. This would also explain why an indirect binding of Atg27-Cub and Nui-Atg23 but not of Atg23-Cub and Nui-Atg27 was detected in the split-ubiquitin assay (see Figure 21 B). However, Nui-Atg21 and Atg27-Cub, where the Cub part faces the cytosol, did clearly interact with each other, suggesting that Atg21 probably interacts with Atg27 on the cytosolic site. Atg9 is a multispanning membrane protein and both termini extend into the cytosol, whereas Atg23 is a cytoplasmic protein, which binds to Atg9 on the cytoplasmic site (Legakis et al., 2007). Atg9 interacts directly with Atg27 and served therefore as positive control for Atg27-Cub. 
Atg23 is a cytosolic protein and interacts indirectly with Atg27 via Atg9. Therefore, Atg23 served as control how an indirect interaction looks like in the split-ubiquitin system (see Figure 21). The interaction of Atg21 with the Cterminal part of Atg27 was indirectly confirmed by truncated versions of Atg27 in the split-ubiquitin assay (see Figure 22). Due to the N-terminal signal sequence only C-terminal truncated versions of Atg27 have been tested. Atg271-198_Cub remained most likely cytosolic, because it was lacking its transmembrane domain. In contrast, neither Atg9 as positive control nor Atg21 interacted with Atg271-221-Cub. This construct was lacking the C-terminus, but still contained the transmembrane domain, supporting an interaction of Atg21 with the C-terminus of Atg27 on the cytosolic site. This would indicate that Atg27 serves as a protein interaction partner to mediate proper membrane association of Atg21. But in fluorescence microscopy the PAS localization of Atg21-YFP had been only slightly influenced by the deletion of ATG27 (see Figure 12). As already explained in more detail, Atg27 could alternatively mediate the PAS localization of Atg21 together with an additional component. Only the simultaneous deletion of both components would then disturb the Atg21 PAS localization (see chapter 5.1).

Furthermore, the colocalization of cAtg27-GFP and mCherry-Atg21 was analyzed in direct fluorescence microscopy to support a potential interaction of both proteins (see Figure 23). Indeed, in more than half of the cells at least $50 \%$ of the mCherry-Atg21 dots colocalized with cAtg27-GFP. This colocalization rate corresponds perfectly to the colocalization rate obtained for Atg27 and Atg9 respectively Atg23 in previous studies (Legakis et al., 2007), supporting a potential interaction of Atg21 and Atg27. Atg1 is required for the retrograde movement of the all three proteins Atg9, Atg23 and Atg27 from the PAS to a peripheral site. Thus all three proteins should be restricted to the PAS by the loss of Atg1 (Legakis et al., 2007; Yen et al., 2007). The colocalization of cAtg27-GFP and mCherry-Atg21 was obtained in atg1د cells to test if both proteins colocalized at the PAS (data not shown). Surprisingly, cAtg27-GFP still formed several dots within the cell comparable to wild type cells. The successful deletion of ATG1 was verified by control PCR analysis and ApeI maturation defect (data not shown). In addition, cAtg27-GFP was completely functional (Figure $23 \mathrm{~A}$ ), 
excluding a mislocalization of cAtg27-GFP. This discrepancy in the localization pattern might be caused by improved microscope sensitivity.

Yeast cells commonly exhibit only one PAS. However, in several cells, all or at least most of the mCherry-Atg21 dots colocalized with cAtg27-GFP (see Figure 23), suggesting that both proteins probably colocalized at multiple sites within the cell. It has to be clarified if one of these sites corresponds to the PAS. A colocalization respectively interaction of both proteins at a site different from the PAS would also explain, why the deletion of ATG27 had only a slight effect on the PAS localization of Atg21-YFP (see Figure 12).

However, Atg27 cycles between the PAS, Golgi and Mitochondria (Kakuta et al., 2012; Yen and Klionsky, 2007), whereas Atg21 localizes to endosomes, the vertices of vacuolar junctions and the PAS (Krick et al., 2008a; Meiling-Wesse et al., 2004)(this study). Determination of the colocalization site of Atg21 and Atg27 could give further information about a potential interaction of both and its physiological relevance. As mentioned before, Atg9-containing vesicles probably directly contribute to the formation of the phagophore (Mari et al., 2010; Yamamoto et al., 2012). Atg27 is required for the anterograde movement of Atg9 to the PAS and vice versa. Thus, Atg27 is also present on the Atg9-containing vesicles and therefore probably involved in the membrane delivery to the PAS at least under nutrient-rich conditions (Kakuta et al., 2012). Moreover, the retrograde moving of Atg9 and Atg27 from the PAS to their peripheral sites occurs independently from each other, suggesting that these proteins may not necessarily cycle together (Legakis et al., 2007). In addition to the Atg1-Atg13 complex, which is needed for the retrograde moving of both Atg9 and Atg27, the Atg21 homologue Atg18 and its interaction partner Atg2 are required for the retrograde moving of Atg9, but not of Atg27 (Yen et al., 2007). Cycling of Atg27 is required for its proper function in Cvt formation. This led to the idea that Atg21 might be involved in the retrograde cycling of Atg27. The loss of Atg21 would lead to a defect of Atg27 cycling and therefore of the membrane delivery under growing conditions resulting in a defect in Cvt vesicle formation, a phenotype that is observed for both proteins. However, this is highly speculative and should be further analyzed. The aspect that Atg27 cycling is independent of Atg14 (Yen et al., 2007), which is in turn needed for proper Atg21 PAS localization (see 
4.2.1), argues against that hypothesis. A localization study of Atg27 in the absence of Atg21 will shed light on this question. As a next step, the potential interaction of both proteins, suggested by the split-ubiquitin assay and the fluorescence microscopy, should be confirmed. In this study, pull down or GFPTRAP $^{\circledR}$ analysis failed to detect a potential interaction of Atg27 and Atg21 (see Figure 24). Atg27 is a membrane protein. Thus, it is possible, that Atg27 was not properly solubilized under the chosen conditions. The cell lysis procedure could be improved and different detergents could be tested to detect a potential interaction with Atg21 as a prerequisite for further functional studies.

\subsection{Interaction studies of Atg21 and Atg8}

The small ubiquitin-like protein Atg8 represents one of the core autophagy proteins and plays dual roles in autophagy. It is required for the expansion of the phagophore. In addition, Atg8 links the cargo to the forming autophagosome by binding to the receptor protein and helps therefore in cargo recognition during selective types of autophagy. To fulfill its function, Atg8 has to be lipidated to PE in a series of conjugation reactions similar to protein ubiquitination (Nakatogawa, 2013) (see chapter 2.2.4.4). Atg21 is required for efficient lipidation of Atg8 and for its recruitment to the PAS, but the underlying mechanism remains elusive (Meiling-Wesse et al., 2004; Stromhaug et al., 2004). In this study, a potential interaction of Atg21 and Atg8 was detected in the splitubiquitin assay (see Figure 25). Bimolecular Fluorescence Complementation (BiFC) was used to further verify a potential interaction of both proteins. As a positive control, cells expressing N-YNAtg8 and its processing enzyme $\mathrm{N}-\mathrm{YC}$ Atg4 were used. Unfortunately, the resulting eYFP-Atg8-Atg4 complex completely located to the nucleus (see Figure $26 \mathrm{C}$ ). Since none of the proteins has been detected in the nucleus before, they were probably completely mislocated. Both constructs were expressed from a constitutive $A D H$ promoter and therefore highly overexpressed, which may have caused the mislocalization of eYFP-Atg4-Atg8. In contrast, the expression of N-YN Atg8 and Atg21 C-YC resulted in perivacuolar dots, which colocalized with the PAS marker ApeI-RFP in a high frequency (60\%) (see Figure $26 \mathrm{E}$ ). This suggests a potential interaction of Atg21 and Atg8 at the PAS. Cells expressing Atg21 C-YC and N-YN alone were 
used as a negative control. Surprisingly, those cells also showed a perivacuolar dot similar to cells expressing both proteins (see Figure $26 \mathrm{E}$ ). The formation of those false-positive signals did not change even after improvement of the expression conditions. Taken together, all cells expressing Atg21 C-YC showed a false-positive signal independent of expression partner, level or duration. During $\mathrm{BiFC}$, the assembly of the two halves of eYFP is irreversible and results in a significant stabilization of the eYFP-protein complex. One advantage of this method is the visualization of only weak and therefore hardly detectable interactions (Skarp et al., 2008). In the negative control cells, N-YN alone was most likely evenly distributed throughout the cytosol. In addition, the amount of N-YN was much higher compared to the amount of N-YN Atg8. Thereby, N-YN might have colocalized by chance close enough to Atg21 C-YC so that the eYFPAtg21 complex could reassemble. Due to the irreversible assembly of both eYFP halves, those complexes would be stabilized and detected by fluorescence microscopy as false positive signals. To avoid incidental eYFP-Atg21 complex formation and therefore false-positive signals, N-YN could be fused to a protein with a defined localization that does not interact with Atg21. Cells expressing that fusion protein and Atg21 C-YC should be further analyzed by fluorescence microcopy to prove if they could serve as a suitable negative control.

The split-ubiquitin assay and BiFC are quite similar methods. BiFC directly monitors the reassembly of eYFP, whereas split-ubiquitin indirectly evaluates the reassembly of two ubiquitin halves. In the split-ubiquitin assay, the empty vector was used as negative control. Therefore, it has never been tested, if one half of ubiquitin expressed alone led to a false-positive interaction signal as the YN part in BiFC. In the split-ubiquitin approach, even the difference between direct and indirect interaction is clearly detectable and was therefore used as an internal control (see Figure 21+Figure 30). This increases the reliability of the method. Moreover, a potential interaction detected in split-ubiquitin was only taken as a hint and confirmed by further approaches.

Surprisingly, the initial functionality test of the BiFC constructs revealed a slight ApeI maturation defect in atg8 8 cells simultaneously expressing both N-YN Atg8 and Atg21 C-YC (see Figure 26 D). In contrast, N-YN Atg8 alone complemented the ApeI maturation defect of atg8 cells. Fluorescence microscopy confirmed 
the reassembly of the two halves of eYFP to an Atg8-Atg21-eYFP complex (see Figure $26 \mathrm{E}$ ). As mentioned above, the assembly of eYFP results in an irreversible formation of the Atg8-Atg21-eYFP complex. These data led to the idea that both proteins would have to dissociate at some point to fulfill their function. It could be possible, that a necessary interaction with further Atg proteins is sterically blocked. PI3P clearance by Ymr1 and therefore dissociation of the autophagic machinery from autophagosomal membranes is required for the maturation of the autophagosome (Cebollero et al., 2012). Furthermore, the Atg8 part present on the outer autophagosomal membrane has to be cleaved off by Atg 4 as a prerequisite for the maturation into a fusion-capable autophagosome (Nair et al., 2012; Yu et al., 2012). Nair et al. (2010) speculated, that Atg21 and Atg18 might prevent premature cleavage of Atg8 from PE by regulating the access to Atg8. Due to the irreversible assembly of the eYFP-Atg8-Atg21 complex, the dissociation of Atg21, that would otherwise allow Atg4 to cleave its substrate Atg8, could be blocked. Therefore, the outer part of Atg8 could not be cleaved off, resulting in an at least delay of the Cvt vesicle maturation and therefore ApeI maturation. However, since the effect on the ApeI maturation obtained in this study is quite small, this hypothesis should be further analyzed.

In conclusion, a potential interaction of Atg21 and Atg8 was detected in the splitubiquitin assay. The interaction was also detected in atg $4 \Delta$ and $\operatorname{atg} 5 \Delta$ cells, where the lipidation of Atg8 is blocked (Figure 25). This pointed to a specific interaction of Atg21 with unlipidated Atg8. Furthermore, recent studies identified WD40 propeller as a new class of ubiquitin binding proteins (Pashkova et al., 2010), supporting an interaction of Atg21 with the ubiquitinlike Atg8. Atg21 is required for efficient lipidation of Atg8 and its recruitment to the PAS by an unknown mechanism (Meiling-Wesse et al., 2004; Stromhaug et al., 2004). The fact that Atg21 interacted with unlipidated Atg8 in the split-ubiquitin assay improves a potential recruitment function for Atg21 in Atg8 lipidation. Nevertheless, as a prerequisite for further functional studies, an interaction of both proteins has to be confirmed by further approaches as pull down or GFPTRAP ${ }^{\circledR}$ S. Almost all Atg8-interacting proteins contain a specific WXXL motif, called Atg8-interacting motif (AIM). This binding motif is specific for Atg8 homologues, since the side chains of the tryptophan and the lysine within the 
AIM bind to hydrophobic pockets in Atg8. These pockets are conserved among all Atg8 homologues but not among other ubiquitin-like proteins (Noda et al., 2010). In addition, Atg8 contains a N-terminal helical domain (NHD) that is absent in ubiquitin. This NHD is required for efficient autophagy, since truncation or deletion of this domain affects autophagy (Nakatogawa et al., 2007). Furthermore, the residues phenylalanine at position 5 and lysine at position 6 within the NHD are essential for binding of at least one interaction partner, Shp1 (Krick et al., 2010). However, it would be interesting to further elucidate, if Atg21 contains an AIM or if the interaction of both proteins is mediated by the NHD as it was shown for interaction with Shp1.

\subsection{Interaction studies of Atg21 and Atg16}

Atg16 is a small coiled-coil protein, which forms a complex with the Atg12-Atg5 conjugate via non-covalently binding to Atg5. This complex plays a crucial role as an E3-like enzyme in the ubiquitin-like conjugation reaction of Atg8 to PE. Most likely, Atg12 directly binds and activates the E2 enzyme Atg3. Therefore, Atg12Atg5/Atg16 probably acts as a platform to bring the Atg8-carrying Atg3 into close proximity to the substrate PE (see chapter 2.2.4.4). The Atg12-Atg5 conjugate was shown to accelerate the Atg8-PE conjugation in vitro, revealing that Atg16 is dispensable for the E3-like function of the Atg12-Atg5 conjugate (Hanada et al., 2007). In vitro Atg5 seems to be able to bind to lipids, but it fails to recruit the Atg12-Atg5 conjugate to the autophagic membranes in vivo (Romanov et al., 2012). Consequently, in vivo Atg16 is required for the membrane recruitment of the Atg12-Atg5/Atg16 complex. In addition, the lipidation of Atg8 is severely impaired in $\operatorname{atg} 16 \Delta$ cells, suggesting that the membrane recruitment of the complex by Atg16 is crucial for its E3-like function (Hanada et al., 2007). The Atg16-mediated PAS localization is dependent on Atg14 and therefore on the PAS-specific PI3P pool. But Atg16 does not contain an obvious PI3P binding motif (Cebollero et al., 2012; Matsushita et al., 2007; Suzuki et al., 2001). Therefore, the underlying mechanism for Atg16 mediated membrane association remains elusive.

The Atg12-Atg5/Atg16 complex is only transiently located at the PAS. For detection via fluorescence microscopy, its PAS localization has to be enriched by 
gene deletions that affect the autophagic flux (Stromhaug et al., 2004). Stromhaug et al. (2004) showed, that the PAS localization of Atg5-YFP in such an Atg8 deletion strain is prevented by the additional loss of Atg21 under growing conditions. In addition, it was reported previously, that the perivacuolar puncta formation of Atg16-GFP was significantly decreased in atg18 $\operatorname{atg} 21 \Delta$ cells under starvation conditions (Nair et al., 2010). In this study, growing atg84 cells and atg8 atg21 cells expressing Atg16-GFP and ApeI-RFP were analyzed using direct fluorescence microscopy to test, if Atg21 had also an influence on the Atg16 PAS localization under growth conditions (see Figure 31), since Atg21 is required for the Cvt pathway. Indeed, the absence of Atg21 led to a significant reduction in the Atg16-GFP PAS rate, that was comparable to the reduction in the Atg5-YFP PAS rate. These results suggest that the Atg16 PAS localization is also dependent on Atg21 under growing conditions (see Figure 31). Furthermore, mCherry-Atg21 colocalized with both Atg16-GFP and Atg5-YFP. All of the Atg16GFP dots and almost all of the Atg5-YFP dots colocalized with the PAS marker ApeI-RFP under those conditions, suggesting that their colocalization with mCherry-Atg21 occured at the PAS (see Figure 32). The colocalization of mCherry-Atg21 and Atg5-YFP was abolished in the absence of Atg16, whereas the colocalization of mCherry-Atg21 and Atg16-GFP was independent on the absence of Atg5. Taken together, the PAS localization of Atg5 depends on both, Atg21 and Atg16 as reported before (Suzuki et al., 2001; Stromhaug et al., 2004), whereas the presence of Atg16 at the PAS depends on Atg21. These data suggests that Atg21 mediates the membrane association of the Atg12Atg5/Atg16 complex under growing conditions. In this study, it was already shown, that part of Atg21 localizes to the PAS by binding to PI3P (see chapter 5.1). Therefore, the recruitment of the Atg12-Atg5/Atg16 complex to the PAS mediated by binding of Atg16 to Atg21 would explain both, the dependence on Atg21 and on Atg14 respectively the PAS-specific PI3P pool for proper Atg12Atg5/Atg16 complex localization. Therefore, a potential interaction of Atg21 and Atg16 was analyzed via additional approaches. Pull down experiments using purified recombinant 6xHis-SUMO-Atg21 incubated with yeast crude cell extract further supported an interaction of Atg21 and Atg16. In this approach, 6xHisSUMO-Atg21, but not 6xSUMO alone, specifically co-precipitated Atg16-HA (see 
Figure 33 B). Furthermore, Atg16-HA specifically bound to GFP-Atg21 but not to GFP alone in GFP-TRAP ${ }^{\circledR} \mathrm{S}$ (see Figure $33 \mathrm{C}$ ). Within the E3-like complex Atg12 interacts with the E2 enzyme Atg3 (Noda et al., 2012). In this study, a potential interaction of Atg21 and Atg8 was already shown. Therefore, it could be possible, that Atg16 is co-precipitated via the bridging protein Atg8. That possibility was excluded in a GFP-TRAP ${ }^{\circledR}$ performed with the crude cell extract of atg8 $\Delta$ cells. Atg16-HA still specifically interacted with GFP-Atg21, supporting a direct interaction (see Figure $33 \mathrm{C}$ ). The split-ubiquitin assay detected an interaction of Atg21 with Atg16 (see Figure 30). In contrast, Atg21 interacted neither with Atg5 nor Atg12, supporting an exclusive binding to Atg16. Finally, direct pull down experiments using recombinant proteins were performed to confirm a direct interaction of both proteins. Indeed, purified GST-Atg16 bound specifically to purified 6xHis-SUMO-Atg21, whereas GST alone did not (see Figure $33 \mathrm{D}$ ). Taken together, Atg16 most likely binds directly to Atg21.

Atg16 consists of a N-terminal Atg5 binding region that is followed by a short linker region and a C-terminal coiled-coil domain (Fujioka et al., 2010) (see Figure $34 \mathrm{~A}$ ). Analysis of truncated versions of Atg16 revealed in pull down and GFP-TRAP ${ }^{\circledR} \mathrm{S}$ that the coiled-coil domain of Atg16 is required for the interaction with Atg21 (see Figure $34 \mathrm{C}$ ). In GFP-TRAP ${ }^{\circledR}$ S, both Atg16 lacking the Atg5binding region (Atg16 $6^{58-150-H A}$ ) or the extreme C-terminus (Atg161-119-HA), that is not required for the coiled-coil formation, still bound to GFP-Atg21. Atg16 1-57. HA was rather unstable and therefore hardly detectable on the immunoblot. However, even overexposed, no bound Atg161-57-HA could be detected. These results suggest a specific Atg21 binding region within the coiled-coil domain of Atg16. Pull down experiments in principle supported those findings (see Figure $34 \mathrm{~B})$. In contrast to Atg161-119-HA in the GFP-TRAP ${ }^{\circledR} \mathrm{S}, \mathrm{GST}-\mathrm{Atg} 16^{1-119}$ did not bind to 6xHis-SUMO-Atg21. It cannot be excluded, that the coiled-coil domain might be longer than proposed (Fujioka et al., 2010). Therefore, the heterologous expression of GST-Atg161-119 in E. coli might lead to an incorrect folding of the protein fragment. However, the direct interaction of Atg16 and Atg21 is probably mediated by a region in the C-terminal coiled-coil domain of Atg16.

The coiled-coil domain is crucial for dimerization respectively formation of the Atg12-Atg5/Atg16 complex and is therefore required for its autophagic function 
(Kuma et al., 2002). Moreover, Fujioka et al. (2010) identified conserved surface residues within the coiled-coil domain of Atg16, that are dispensable for the formation of the coiled-coil structure, but are strictly required for the Cvt pathway and macroautophagy under both growing and starvation conditions. As mentioned before, the PAS recruitment of the Atg12-Atg5/Atg16 complex is a prerequisite for its E3-like function in autophagy (Hanada et al., 2007). Results from this study propose that the PAS recruitment of the complex is probably mediated by binding of Atg21 to the coiled-coil domain of Atg16. Therefore, the conserved residues in the coiled-coil domain of Atg16 that are crucial for autophagy, could mediate the interaction with Atg21. Two conserved Atg16 residues, the aspartic acid at position 101 (D101) and the glutamic acid at position 102 (E102) were mutated either alone or in concert to alanine and the interaction with Atg21 was tested (see Figure 36). Indeed, both amino acid substitutions severely affected the interaction with GFP-Atg21 in GFP-TRAP ${ }^{\circledR}$ S. Interestingly, the interaction of Atg16 ${ }^{\mathrm{E} 102 \mathrm{~A}}-\mathrm{HA}$ with GFP-Atg21 was significantly

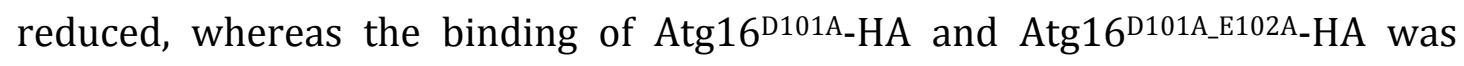
completely abolished, suggesting a stricter requirement of amino acid D101 for the interaction with Atg21. Consequently, Atg21-binding deficient Atg16-GFP mutants were not longer able to localize to the PAS and were completely cytosolic in fluorescence microscopy (see Figure 35). Interestingly, only Atg16 ${ }^{\mathrm{E} 102 \mathrm{~A}}$-GFP showed a rare and very faint perivacuolar dot in the same cells, supporting a stricter requirement of D101 for the Atg21 interaction than E102. The possibility, that the mutations of these amino acids disturb the structure of the Atg16 coiled-coil domain leading to a misfolded protein unable to bind Atg21, could be excluded for two reasons. First, the coiled-coil domain of Atg16 mediates dimerization of Atg16 and the ability of the Atg16 mutants to dimerize was verified in GFP-TRAP ${ }^{\circledR}$ S (see Figure 36 B). Second, Fujioka et al. (2010) used the Atg16 ${ }^{\text {D101A_E102A }}$ mutant to crystalize the coiled-coil domain of Atg16. This would have been impossible with mutations in Atg16 that affect the coiled-coil formation.

Taken together, the conserved surface residues E102 and more important D101 in the coiled-coil domain of Atg16 mediate the interaction with Atg21. Mutations of these residues disturb the binding to Atg21 and result in a severe Cvt pathway 
and autophagy defect (this study,(Fujioka et al., 2010)), indicating that the interaction with Atg21 is needed for the autophagic function of Atg16. In detail, the autophagic function of Atg16 is represented by its role in the E3-like Atg12Atg5/Atg16 complex during lipidation of Atg8. Therefore, the ability of the E3 complex containing the Atg21-binding deficient Atg16 mutants to efficiently conjugate Atg8 to PE was tested under growing conditions (see Figure 37). Indeed, the lipidation of Atg8 was significantly retarded in atg16 cells expressing the Atg21-binding defective mutants (Atg16 ${ }^{\mathrm{D} 101 \mathrm{~A}}-\mathrm{HA}, \mathrm{Atg} 16^{\mathrm{E} 102 \mathrm{~A}}-\mathrm{HA}$

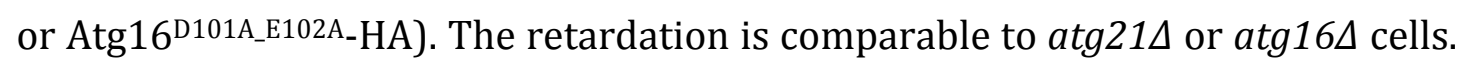
It can be excluded, that the HA-tag influenced the functionality of Atg16-HA, since the Atg16 wild type construct fully complemented both the ApeI maturation and Atg8 lipidation defect in atg16 cells. Consequently, the Atg21binding deficient Atg16 mutants showed an additional ApeI maturation defect as previously described by Fujioka et al. (2010). Interestingly, D101A had a stronger effect on the ApeI maturation as E102A, supporting a stronger requirement for the interaction with Atg21. In contrast to the GFP-TRAP ${ }^{\circledR}$ results, the double mutation D101A_E102A exhibited the strongest effect and caused a complete block in ApeI maturation. This further supports that both amino acids contribute to the interaction with Atg21 in vivo.

Atg8 lipidation and ApeI maturation were also tested in atg16 cells expressing the truncated versions of Atg16 (see Figure 37). Atg8 lipidation and ApeI maturation was abolished in cells expressing Atg161-57-HA, most likely because this construct was not stable in yeast cells resulting in an atg16 phenotype. Furthermore, Atg8 lipidation was impaired in atg16 cells expressing Atg16 58-150-HA. This truncated Atg16 version is still able to bind to Atg21 as seen in GFP-TRAP ${ }^{\circledR} \mathrm{S}$, but the Atg5-binding domain is lacking. Therefore, the E3-like Atg12-Atg5/Atg16 complex could not be formed resulting in an Atg8 lipidation and ApeI maturation defect. $\operatorname{atg} 16 \Delta$ cells expressing Atg161-119-HA exhibited an ApeI maturation defect as described before (Fujioka et al., 2010). Interestingly, those cells additionally showed an impaired Atg8 lipidation. This construct is able to bind to Atg21 as seen in GFP-TRAP ${ }^{\circledR}$ S (see Figure 34) and still contains the Atg5-binding region, suggesting that the extreme C-terminal domain of Atg16 
might be involved in Atg8 lipidation and autophagy in a mechanism independent on formation of the Atg12-Atg5/Atg16 complex and binding to Atg21.

Taken together, the interaction of Atg16 and Atg21 mediated by the residues D101 and E102 within the coiled-coil region in Atg16 is required for efficient Atg8 lipidation and therefore efficient ApeI transport.

One of the control mutants Atg16 ${ }^{\mathrm{K} 94 \mathrm{~A}}$-HA showed an unexpected autophagic activity under Cvt conditions in these studies. Originally, it was reported, that K94 and E97 have no influence on the autophagic activity of Atg16 (Fujioka et al., 2010). Therefore, mutations to alanine of both residues were selected as negative controls. Interestingly, the interaction of Atg16 ${ }^{\mathrm{K} 94 \mathrm{~A}}-\mathrm{HA}$ and $\mathrm{Atg} 21$ was severely impaired in GFP-TRAP ${ }^{\circledR} \mathrm{S}$, comparable to the defect seen with Atg21 and Atg16 ${ }^{\mathrm{E} 102 \mathrm{~A}}$-HA (see Figure $36 \mathrm{C}$ ). Consequently, as interaction of Atg21 and Atg16 is proposed to be essential for Atg8 lipidation reaction, the formation of Atg8-PE was significantly retarded in atg16 cells expressing Atg16 ${ }^{\mathrm{K} 94 \mathrm{~A}}$-HA (see Figure 37). Surprisingly, the ApeI maturation occurred normally in these cells, corresponding to results from Fujioka et al. (2010). In addition, the ability of $\operatorname{Atg} 16^{\mathrm{K} 94 \mathrm{~A}}$ to dimerize was confirmed by GFP-TRAP ${ }^{\circledR} \mathrm{S}$ (see Figure $36 \mathrm{~B}$ ), excluding a structural defect of the mutant. Taken together, these cells exhibited normal Cvt pathway independent on lipidated Atg8. The lipidation of Atg8 was not completely blocked in these cells (see Figure 37), suggesting that the residual amount of Atg8-PE might be sufficient for Cvt vesicle formation. However, the amount of lipidated Atg8 was comparable to that of other Atg21-binding deficient Atg16 mutants, where ApeI maturation is blocked (see Figure 37). The mechanism that allows ApeI maturation without proper Atg8 lipidation in cells expressing Atg16 ${ }^{\mathrm{K} 94 \mathrm{~A}}-\mathrm{HA}$ remains to be elucidated.

Proposed model:

One elementary result of this study represents the detection of Atg21 at the PAS under growing conditions. This is the first time that Atg21 has been detected at the PAS (see Figure 11). The Atg21 PAS localization is dependent on Atg14 and thus on the PAS-specific PI3P pool. Importantly, Atg21 recruits the Atg12Atg5/Atg16 complex to the PAS via direct interaction with the coiled-coil region of Atg16 (see chapter 4.3.6.4). Thereby the underlying mechanism of PI3P- 
dependent plus Atg16-mediated recruitment of the Atg12-Atg5/Atg16 complex to autophagosomal membranes has been elucidated in this study. More importantly, the interaction of Atg21 and Atg16 is not only a prerequisite for the proper PAS localization of the Atg12-Atg5/Atg16 complex but also for its autophagic function (see chapter 5.7). Consequently, interaction of Atg16 and Atg21 is needed for efficient Atg8 lipidation (see Figure 37), providing a reasonable explanation for the proposed function of Atg21 in Atg8 lipidation (Meiling-Wesse et al., 2004; Stromhaug et al., 2004).

Interestingly, also Atg21 and Atg8 probably interact with each other (chapter 5.6). This interaction occurs even if Atg8 is unlipidated. Taken together, these data suggest, that Atg21 not only mediates the membrane association of the E3 enzyme Atg12-Atg5/Atg16, but also acts as a scaffold for the Atg8 lipidation reaction by mediating interaction of activated Atg8 and its E3-enzyme Atg12-Atg5/Atg16. Importantly, this model provides a reasonable explanation for the fundamental role of Atg21 in Cvt vesicle formation.

The Atg12-Atg5/Atg16 complex was proposed to specify the lipidation site of Atg8 via association with Atg8-carrying Atg3 and autophagosomal membranes (Hanada et al., 2007). However, it was unclear how Atg16 mediates the membrane association of the E3-like complex to determine the site of Atg8 lipidation. Regarding the proposed model, Atg21 represents the missing link that mediates PI3P-dependent membrane association of the lipidation machinery by recruiting the activated substrate Atg8 and the E3 enzyme required for the last step of Atg8 lipidation. Thereby, Atg21 might represent the key factor to specify the lipidation site.

In the absence of Atg8, Atg21 and the otherwise hardly detectable Atg12Atg5/Atg16 complex colocalize at the PAS (see Figure 32), suggesting that the lack of Atg8 leads to the accumulation of both proteins. This would however indicate that Atg8 is lipidated at the PAS. In fact, it is still highly under debate, where the Atg8 lipidation occurs within the cell. Recent studies suggest, that Atg8 lipidation does not only occur at the PAS or the phagophore in response to autophagy-inducing signals but in addition constitutively and non-selectively at other sites in the cell (Nair et al., 2012; Nakatogawa et al., 2012; Yu et al., 2012). 
These results have been obtained in a strain lacking Atg4, leading to an artificial situation, where Atg8 cannot be delipidated. Furthermore, a model has been proposed, where Atg4 should subsequently deconjugate the Atg8-PE on inappropriate membranes, but not from the PAS, where it is protected against delipidation. This should maintain a cytoplasmic pool of unlipidated Atg8 that can undergo lipidation and participate in autophagosome formation the PAS (Nakatogawa et al., 2012). The mechanism that should restrict and regulate Atg 4 activity is not known. As mentioned before, Nair et al. (2010) speculated that both, Atg18 and Atg21, prevent premature cleavage of Atg8 from PE via regulating the access to Atg8. It remains to be determined, if Atg21 and Atg12Atg5/Atg16 were involved in the lipidation of Atg8 at another site than the PAS or if they were involved in the protection of Atg8 from cleavage by Atg4 as discussed before (see chapter 5.6). However, results from this study question the proposed model of a ubiquitous Atg8 lipidation within the cell. In the absence of Atg8, the Atg12-Atg5/Atg16 complex was restricted specifically to the PAS and no other site in the cell.

As already mentioned, recent studies revealed that Atg5 alone is capable to bind membranes in vitro. Fractionation experiments using different yeast deletion strains supported that Atg5 partially mediates membrane binding also in vivo (Romanov et al., 2012). Surprisingly, this membrane binding is inhibited by Atg12 alone, but recovered upon binding of Atg16 to the Atg12-Atg5 conjugate, indicating that Atg16 regulates association of the Atg12-Atg5 conjugate with membranes in vivo (Romanov et al., 2012). This study now revealed that the Atg12-Atg5/Atg16 membrane association is mediated via interaction of Atg16 with Atg21 (see chapter 5.7). The formation of the Atg12-Atg5 conjugate seems unaffected in $\operatorname{atg} 21 \Delta$ cells (Stromhaug et al., 2004). Atg16 and the Atg12-Atg5 conjugate could either form a complex in the cytosol, which is subsequently recruited to the PAS or Atg16 localizes independently to the PAS via binding to Atg21 and subsequently recruits the Atg12-Atg5 conjugate to that site. In the latter case, the binding of Atg21 to Atg16 would have to stimulate its interaction with the Atg12-Atg5 conjugate by an unknown mechanism to preserve the Atg12-Atg5/Atg16 complex formation in the cytosol. In another model, it is also possible, that Atg21 as a WD40 propeller facilitates the Atg12-Atg5/Atg16 
complex formation by binding both, Atg16 and the Atg12-Atg5 conjugate. As mentioned above, WD40 propeller were identified to bind to ubiquitin-like proteins (Pashkova et al., 2010). Atg12 contains one (Suzuki et al., 2005), whereas Atg5 exhibits two ubiquitin-like folds (Matsushita et al., 2007). As already shown before, Atg21 probably binds to Atg8, supporting the ability of Atg21 to bind to ubiquitin-like proteins. In contrast, the split-ubiquitin assay detected a specific interaction of Atg21 with Atg16, but neither with Atg12 nor Atg5 (see Figure 30), arguing against a potential additional interaction with Atg5 and/or Atg12. Anyhow, an interaction of Atg21 with Atg5 or Atg12 should be analyzed by further approaches like pull down or GFP-TRAP ${ }^{\circledR}$ S. Furthermore, the formation of the Atg12-Atg5/Atg16 complex can be visualized by gelfiltration chromatography as an approximately $350 \mathrm{kDa}$ complex (Kuma et al., 2002). The complex is most likely dimeric mediated by Atg16 (Fujioka et al., 2010). In the absence of Atg16, the Atg12-Atg5 conjugate remains monomeric and would be easily distinguishable from the Atg12-Atg5/Atg16 complex in gelfiltration chromatography. Therefore, determining the molecular weight range of the Atg12-Atg5 conjugate in atg21د cells by gelfiltration chromatography, should reveal an influence of Atg21 on the formation of the Atg12-Atg5/Atg16 complex. Furthermore, the Atg16- and Atg8-interacting site within the WD40 propeller Atg21 should be identified. WD40 propeller are commonly known to serve as a large interaction platform to mediate protein-protein interactions. Mapping of the Atg16- and Atg8-interacting site within Atg21 would elucidate the constitution of the proposed Atg8 lipidation complex. It is possible, that Atg21 recruits additional proteins of the Atg8 conjugation machinery in order to further facilitates the lipidation reaction. A candidate for additional interaction with Atg21 would be at least the E2 enzyme Atg3, which carries the activated Atg8. Atg12 directly binds and most likely activates Atg3 (Hanada et al., 2007; Sakoh-Nakatogawa et al., 2013). Thereby, Atg8 and Atg16 are already indirectly connected via the Atg12-Atg5 conjugate bound to Atg3. It can not be excluded that a simultaneous binding of Atg8 and Atg16 is not possible due to steric hinderance. As mentioned above, Atg5 is able to partially mediate membrane association after initial recruitment of Atg16 to autophagosomal membranes (Romanov et al., 2012). Therefore, it could be speculated, that Atg21 is only 
needed for the initial recruitment of the Atg12-Atg5/Atg16 complex to the autophagosomal membranes. Afterwards the complex would be able to remain membrane associated mediated by Atg5 and a simultaneous binding of Atg21 to Atg16 and Atg8 would not be absolutely necessary. However, identification of the Atg16- and Atg8-interacting site within Atg21 should shed light on this question. Chopping Atg21 in pieces, that could be used as Atg21 versions in GFP$\mathrm{TRAP}^{\circledR} \mathrm{S}$ or pull downs, is not possible, because of the rigid propeller fold. Alternatively, competition of short Atg21 peptides representing potential binding sites with full length Atg21 in pull down experiments with Atg8 or Atg16 could elucidate the binding site within Atg21.

In this study, the conserved surface residues D101 and E102 within the coiledcoil region of Atg16 were identified to bind Atg21 (see Figure 36). These residues originate from a set of mutants, that Fujioka et al. (2010) have tested for a defect in the Cvt pathway and autophagy. In addition to D101 and E102, mutation of the isoleucines at position 104 and 108 (I104 and I108) alone or in concert caused strong defects in both pathways. It would be interesting to test if these residues were additionally required for an interaction with Atg21. Results from this study and observations from Fujioka et al. (2010) revealed that the extreme C-terminus of Atg16, that is not required for the coiled-coil formation, might have a crucial but yet unidentified role in autophagy independent of Atg21 (see above). Similarly, it could be possible, that the I104 and I108 have a different role than binding to Atg21. Interaction studies with Atg16 mutants using GFP-TRAP ${ }^{\circledR} \mathrm{S}$ or further approaches should shed light on this question.

Atg21 is required for the Cvt pathway. All approaches in this study were therefore performed under growing conditions. Bulk autophagy is also severely affected in the absence of Atg21 under autophagy-inducing conditions, but not completely blocked (Barth et al., 2002; Stromhaug et al., 2004). Similar, Atg21 is required for the PAS recruitment of Atg8 only under growing conditions, but $\operatorname{atg} 21 \Delta$ cells accumulate unlipidated Atg8 even after $4 \mathrm{~h}$ of nitrogen starvation (Stromhaug et al., 2004). The lipidation is not completely blocked, but severely delayed. The amount of Atg8 is highly upregulated under autophagy-inducing conditions, suggesting that the small part of Atg8, that is lipidated in the absence of Atg21, is sufficient to be detected at the PAS and to maintain a particular level 
of bulk autophagy. However, the fact that Atg8 lipidation is impaired in the absence of Atg21 even under starvation, suggests that Atg21 is also needed for the recruitment of the Atg12-Atg5/Atg16 complex under those conditions. In contrast, Nair et al. (2010) observed perivacuolar Atg16-GFP puncta formation after $4 \mathrm{~h}$ starvation in wild type and $\operatorname{atg} 21 \Delta / \operatorname{atg} 18 \Delta$ single or $\operatorname{atg} 21 \Delta \operatorname{atg} 18 \Delta$ double deletion cells. Only the simultaneous loss of both Atg18 and Atg21 resulted in a significant reduction in the Atg16-GFP PAS localization (Nair et al., 2010), suggesting that both proteins are required in concert for the Atg12Atg5/Atg16 recruitment under starvation conditions. However, this finding seems to disagree with the observation that the loss of Atg18 has no effect on the Atg8 recruitment to the PAS or its lipidation, neither under growing nor under starvation conditions (Stromhaug et al., 2004; Suzuki et al., 2007). Further investigation on the specific role of each homologue under both growing and starvation conditions should shed light on these discrepancies. 


\section{Bibliography}

Alemu, E. A., Lamark, T., Torgersen, K. M., Birgisdottir, A. B., Larsen, K. B., et al. (2012). ATG8 family proteins act as scaffolds for assembly of the ULK complex: sequence requirements for LC3-interacting region (LIR) motifs. Journal of Biological Chemistry 287, 39275-39290.

Araki, Y., Ku, W. C., Akioka, M., May, A. I., Hayashi, Y., Arisaka, F., Ishihama, Y. and Ohsumi, Y. (2013). Atg38 is required for autophagy-specific phosphatidylinositol 3-kinase complex integrity. J Cell Biol 203, 299-313.

Baba, M., Takeshige, K., Baba, N. and Ohsumi, Y. (1994). Ultrastructural analysis of the autophagic process in yeast: detection of autophagosomes and their characterization. J Cell Biol 124, 903-913.

Barth, H., Meiling-Wesse, K., Epple, U. D. and Thumm, M. (2001). Autophagy and the cytoplasm to vacuole targeting pathway both require Aut10p. FEBS Letters 508, 23-28.

Barth, H., Meiling-Wesse, K., Epple, U. D. and Thumm, M. (2002). Mai1p is essential for maturation of proaminopeptidase I but not for autophagy. FEBS Letters 512, 173-179.

Baskaran, S., Ragusa, M. J. and Hurley, J. H. (2012). How Atg18 and the WIPIs sense phosphatidylinositol 3-phosphate. Autophagy 8, 1851-1852.

Budovskaya, Y., Stephan, J., Deminoff, S. and Herman, P. (2005). An evolutionary proteomics approach identifies substrates of the cAMP-dependent protein kinase. Proc Natl Acad Sci U S A 102, 13933-13938.

Cebollero, E., der Vaart, van, A., Zhao, M., Rieter, E., Klionsky, D. J., Helms, J. B. and Reggiori, F. (2012). Phosphatidylinositol-3-phosphate clearance plays a key role in autophagosome completion. Curr Biol 22, 1545-1553.

Chang, C.-Y. C. and Huang, W.-P. W. (2007). Atg19 mediates a dual interaction cargo sorting mechanism in selective autophagy. Mol Biol Cell 18, 919-929.

Chen, Y. and Klionsky, D. J. (2011). The regulation of autophagy - unanswered questions. J Cell Sci 124, 161-170.

Cheong, H., Nair, U., Geng, J. and Klionsky, D. J. (2008). The Atg1 kinase complex is involved in the regulation of protein recruitment to initiate sequestering vesicle formation for nonspecific autophagy in Saccharomyces cerevisiae. Mol Biol Cell 19, 668-681.

Chew, L. H., Setiaputra, D., Klionsky, D. J. and Yip, C. K. (2013). Structural characterization of the Saccharomyces cerevisiae autophagy regulatory complex Atg17-Atg31-Atg29. Autophagy 9.

Choi, A. M. K., Ryter, S. W. and Levine, B. (2013). Autophagy in human health and disease. N. Engl. J. Med. 368, 1845-1846.

Dove, S. K., Dong, K., Kobayashi, T., Williams, F. K. and Michell, R. H. (2009). Phosphatidylinositol 3,5-bisphosphate and Fab1p/PIKfyve underPPIn endo-lysosome function. Biochem J 419, 1.

Dove, S., Piper, R., McEwen, R., Yu, J., King, M., Hughes, D., Thuring, J., Holmes, A., Cooke, F., Michell, R., et al. (2004). Svp1p defines a family of phosphatidylinositol 3,5-bisphosphate effectors. EMBO J 23, 1922-1933. 
Efe, J., Botelho, R. and Emr, S. (2007). Atg18 regulates organelle morphology and Fab1 kinase activity independent of its membrane recruitment by phosphatidylinositol 3,5-bisphosphate. Mol Biol Cell 18, 4232-4244.

Fujioka, Y., Noda, N. N., Nakatogawa, H., Ohsumi, Y. and Inagaki, F. (2010). Dimeric coiled-coil structure of Saccharomyces cerevisiae Atg16 and its functional significance in autophagy. Journal of Biological Chemistry 285, 1508-1515.

Fujita, N., Itoh, T., Omori, H., Fukuda, M., Noda, T. and Yoshimori, T. (2008). The Atg16L complex specifies the site of LC3 lipidation for membrane biogenesis in autophagy. Mol Biol Cell 19, 2092-2100.

Geng, J., Baba, M., Nair, U. and Klionsky, D. J. (2008). Quantitative analysis of autophagyrelated protein stoichiometry by fluorescence microscopy. J Cell Biol 182, 129-140.

Gillooly, D. J., Morrow, I. C., Lindsay, M., Gould, R., Bryant, N. J., Gaullier, J. M., Parton, R. G. and Stenmark, H. (2000). Localization of phosphatidylinositol 3-phosphate in yeast and mammalian cells. EMBO J 19, 4577-4588.

Goffeau, A., Barrell, B., Bussey, H., Davis, R., Dujon, B., Feldmann, H., Galibert, F., Hoheisel, J., Jacq, C., Johnston, M., et al. (1996). Life with 6000 Genes. Science 1-7.

Guan, J., Stromhaug, P., George, M., Habibzadegah-Tari, P., Bevan, A., Dunn, W. and Klionsky, D. (2001). Cvt18/Gsa12 is required for cytoplasm-to-vacuole transport, pexophagy, and autophagy in Saccharomyces cerevisiae and Pichia pastoris. Mol Biol Cell 12, 3821-3838.

Hanada, T., Noda, N. N., Satomi, Y., Ichimura, Y., Fujioka, Y., Takao, T., Inagaki, F. and Ohsumi, Y. (2007). The Atg12-Atg5 conjugate has a novel E3-like activity for protein lipidation in autophagy. J Biol Chem 282, 37298-37302.

Hanahan, D. (1983). Studies on Transformation of Escherichia coli with Plasmids. J Mol Biol 166, 557-580.

Harding, T., Morano, K., Scott, S. and Klionsky, D. (1995). Isolation and characterization of yeast mutants in the cytoplasm to vacuole protein targeting pathway. J Cell Biol 131, 591602.

He, C. and Klionsky, D. J. (2009). Regulation mechanisms and signaling pathways of autophagy. Annu Rev Genet 43, 67-93.

He, C., Song, H., Yorimitsu, T., Monastyrska, I., Yen, W.-L., Legakis, J. E. and Klionsky, D. J. (2006). Recruitment of Atg9 to the preautophagosomal structure by Atg11 is essential for selective autophagy in budding yeast. J Cell Biol 175, 925-935.

Ho, H., Ogawa, M., Kapadia, R., Yoshikawa, Y., Al-Tahan, S., Kobayashi, T., Ahmad, S., Mimuro, H., Ganesan, A. K., Fukumatsu, M., et al. (2011). WIPI1 coordinates melanogenic gene transcription and melanosome formation via TORC1 inhibition. Journal of Biological Chemistry 286, 12509-12523.

Huang, W. P., Scott, S. V., Kim, J. and Klionsky, D. J. (2000). The itinerary of a vesicle component, Aut7p/Cvt5p, terminates in the yeast vacuole via the autophagy/Cvt pathways. $J$ Biol Chem 275, 5845-5851.

Hutchins, M. and Klionsky, D. (2001). Vacuolar localization of oligomeric alpha-mannosidase requires the cytoplasm to vacuole targeting and autophagy pathway components in Saccharomyces cerevisiae. J Biol Chem 276, 20491-20498.

Ichimura, Y., Kirisako, T., Takao, T., Satomi, Y., Shimonishi, Y., Ishihara, N., Mizushima, N., 
Tanida, I., Kominami, E., Ohsumi, M., et al. (2000). A ubiquitin-like system mediates protein lipidation. Nature 408, 488-492.

Inoue, Y. and Klionsky, D. J. (2010). Regulation of macroautophagy in Saccharomyces cerevisiae. Semin Cell Dev Biol 21, 664-670.

Ishihara, N., Hamasaki, M., Yokota, S., Suzuki, K., Kamada, Y., Kihara, A., Yoshimori, T., Noda, T. and Ohsumi, Y. (2001). Autophagosome requires specific early Sec proteins for its formation and NSF/SNARE for vacuolar fusion. Mol Biol Cell 12, 3690-3702.

Itakura, E. and Mizushima, N. (2010). Characterization of autophagosome formation site by a hierarchical analysis of mammalian Atg proteins. Autophagy 6, 764-776.

Janke, C., Magiera, M., Rathfelder, N., Taxis, C., Reber, S., Maekawa, H., Moreno-Borchart, A., Doenges, G., Schwob, E., Schiebel, E., et al. (2004). A versatile toolbox for PCR-based tagging of yeast genes: new fluorescent proteins, more markers and promoter substitution cassettes. Yeast 21, 947-962.

Jao, C. C., Ragusa, M. J., Stanley, R. E. and Hurley, J. H. (2013). A HORMA domain in Atg13 mediates PI 3-kinase recruitment in autophagy. Proc Natl Acad Sci U S A 110, 5486-5491.

Jeffries, T., Dove, S., Michell, R. and Parker, P. (2004). PtdIns-specific MPR pathway association of a novel WD40 repeat protein, WIPI49. Mol Biol Cell 15, 2652-2663.

Jin, N., Chow, C. Y., Liu, L., Zolov, S. N., Bronson, R., Davisson, M., Petersen, J. L., Zhang, Y., Park, S., Duex, J. E., et al. (2008). VAC14 nucleates a protein complex essential for the acute interconversion of PI3P and PI(3,5)P(2) in yeast and mouse. EMBO J 27, 3221-3234.

Kabeya, Y., Mizushima, N., Ueno, T., Yamamoto, A., Kirisako, T., Noda, T., Kominami, E., Ohsumi, Y. and Yoshimori, T. (2000). LC3, a mammalian homologue of yeast Apg8p, is localized in autophagosome membranes after processing. EMBO J 19, 5720-5728.

Kabeya, Y., Noda, N. N., Fujioka, Y., Suzuki, K., Inagaki, F. and Ohsumi, Y. (2009). Characterization of the Atg17-Atg29-Atg31 complex specifically required for starvationinduced autophagy in Saccharomyces cerevisiae. Biochem Biophys Res Commun 389, 612615.

Kakuta, S., Yamamoto, H., Negishi, L., Kondo-Kakuta, C., Hayashi, N. and Ohsumi, Y. (2012). Atg9 vesicles recruit vesicle-tethering proteins Trs85 and Ypt1 to the autophagosome formation site. Journal of Biological Chemistry 287, 44261-44269.

Kamada, Y., Funakoshi, T., Shintani, T., Nagano, K., Ohsumi, M. and Ohsumi, Y. (2000). Tormediated induction of autophagy via an Apg1 protein kinase complex. J Cell Biol 150, 15071513.

Kamada, Y., Yoshino, K. I., Kondo, C., Kawamata, T., Oshiro, N., Yonezawa, K. and Ohsumi, Y. (2010). Tor Directly Controls the Atg1 Kinase Complex To Regulate Autophagy. Mol Cell Biol 30, 1049-1058.

Kawamata, T., Kamada, Y., Kabeya, Y., Sekito, T. and Ohsumi, Y. (2008). Organization of the pre-autophagosomal structure responsible for autophagosome formation. Mol Biol Cell 19, 2039-2050.

Kihara, A., Noda, T., Ishihara, N. and Ohsumi, Y. (2001). Two Distinct Vps34 Phosphatidylinositol 3-Kinase Complexes Function in Autophagy and Carboxypeptidase Y Sorting in Saccharomyces cerevisiae. J Cell Biol 152, 519-530.

Kim, J. and Klionsky, D. (2000). Autophagy, cytoplasm-to-vacuole targeting pathway, and pexophagy in yeast and mammalian cells. Annu Rev Biochem 69, 303-342. 
Kim, J., Dalton, V., Eggerton, K., Scott, S. and Klionsky, D. (1999). Apg7p/Cvt2p is required for the cytoplasm-to-vacuole targeting, macroautophagy, and peroxisome degradation pathways. Mol Biol Cell 10, 1337-1351.

Kirisako, T., Baba, M., Ishihara, N., Miyazawa, K., Ohsumi, M., Yoshimori, T., Noda, T. and Ohsumi, Y. (1999). Formation process of autophagosome is traced with Apg8/Aut7p in yeast. J Cell Biol 147, 435-446.

Kirisako, T., Ichimura, Y., Okada, H., Kabeya, Y., Mizushima, N., Yoshimori, T., Ohsumi, M., Takao, T., Noda, T. and Ohsumi, Y. (2000). The reversible modification regulates the membrane-binding state of Apg8/Aut7 essential for autophagy and the cytoplasm to vacuole targeting pathway.J Cell Biol 151, 263-276.

Klionsky, D. J., Cregg, J. M., Dunn, W. A., Emr, S. D., Sakai, Y., Sandoval, I. V., Sibirny, A., Subramani, S., Thumm, M., Veenhuis, M., et al. (2003). A unified nomenclature for yeast autophagy-related genes. Developmental Cell 5, 539-545.

Klionsky, D., Cueva, R. and Yaver, D. (1992). Aminopeptidase I of Saccharomyces cerevisiae is localized to the vacuole independent of the secretory pathway. J Cell Biol 119, 287-299.

Knop, M., Siegers, K., Pereira, G., Zachariae, W., Winsor, B., Nasmyth, K. and Schiebel, E. (1999). Epitope tagging of yeast genes using a PCR-based strategy: more tags and improved practical routines. Yeast 15, 963-972.

Kraft, C., Kijanska, M., Kalie, E., Siergiejuk, E., Lee, S. S., Semplicio, G., Stoffel, I., Brezovich, A., Verma, M., Hansmann, I., et al. (2012). Binding of the Atg1/ULK1 kinase to the ubiquitin-like protein Atg8 regulates autophagy. EMBO J 31, 3691-3703.

Krick, R., Bremer, S., Welter, E., Schlotterhose, P., Muehe, Y., Eskelinen, E.-L. and Thumm, M. (2010). Cdc48/p97 and Shp1/p47 regulate autophagosome biogenesis in concert with ubiquitin-like Atg8. J Cell Biol 190, 965-973.

Krick, R., Busse, R. A., Scacioc, A., Stephan, M., Janshoff, A., Thumm, M. and Kühnel, K. (2012). Structural and functional characterization of the two phosphoinositide binding sites of PROPPINs, a $\beta$-propeller protein family. Proc Natl Acad Sci U S A 109, E2042-9.

Krick, R., Henke, S., Tolstrup, J. and Thumm, M. (2008a). Dissecting the localization and function of Atg18, Atg21 and Ygr223c. Autophagy 4, 896-910.

Krick, R., Muehe, Y., Prick, T., Bremer, S., Schlotterhose, P., Eskelinen, E.-L., Millen, J., Goldfarb, D. S. and Thumm, M. (2008b). Piecemeal microautophagy of the nucleus requires the core macroautophagy genes. Mol Biol Cell 19, 4492-4505.

Krick, R., Tolstrup, J., Appelles, A., Henke, S. and Thumm, M. (2006). The relevance of the phosphatidylinositolphosphat-binding motif FRRGT of Atg18 and Atg21 for the Cvt pathway and autophagy. FEBS Letters 580, 4632-4638.

Kuma, A., Mizushima, N., Ishihara, N. and Ohsumi, Y. (2002). Formation of the approximately 350-kDa Apg12-Apg5.Apg16 multimeric complex, mediated by Apg16 oligomerization, is essential for autophagy in yeast. J Biol Chem 277, 18619-18625.

Kvam, E. and Goldfarb, D. (2007). Nucleus-vacuole junctions and piecemeal microautophagy of the nucleus in S. cerevisiae. Autophagy 3, 85-92.

Laemmli, U.K. (1970). Cleavage of structural proteins during the assembly of the head of bacteriophage T4. Nature 227, 680-685.

Lang, T., Schaeffeler, E., Bernreuther, D., Bredschneider, M., Wolf, D. H. and Thumm, M. (1998). Aut2p and Aut7p, two novel microtubule-associated proteins are essential for 
delivery of autophagic vesicles to the vacuole. EMBO J 17, 3597-3607.

Laser, H., Bongards, C., Schuller, J., Heck, S., Johnsson, N. and Lehming, N. (2000). A new screen for protein interactions reveals that the Saccharomyces cerevisiae high mobility group proteins Nhp6A/B are involved in the regulation of the GAL1 promoter. Proc Natl Acad Sci U S A 97, 13732-13737.

Legakis, J. E., Yen, W.-L. and Klionsky, D. J. (2007). A cycling protein complex required for selective autophagy. Autophagy 3, 422-432.

Lipatova, Z., Belogortseva, N., Zhang, X. Q., Kim, J., Taussig, D. and Segev, N. (2012). Regulation of selective autophagy onset by a Ypt/Rab GTPase module. Proc Natl Acad Sci U S A 109, 6981-6986.

Loewith, R. and Hall, M. N. (2011). Target of Rapamycin (TOR) in Nutrient Signaling and Growth Control. Genetics 189, 1177-1201.

Longtine, M., McKenzie, A., Demarini, D., Shah, N., Wach, A., Brachat, A., Philippsen, P. and Pringle, J. (1998). Additional modules for versatile and economical PCR-based gene deletion and modification in Saccharomyces cerevisiae. Yeast 14, 953-961.

Lynch-Day, M. A. and Klionsky, D. J. (2010). The Cvt pathway as a model for selective autophagy. FEBS Letters 584, 1359-1366.

Lynch-Day, M. A., Bhandari, D., Menon, S., Huang, J., Cai, H., Bartholomew, C. R., Brumell, J. H., Ferro-Novick, S. and Klionsky, D. J. (2010). Trs85 directs a Ypt1 GEF, TRAPPIII, to the phagophore to promote autophagy. Proc Natl Acad Sci U S A 107, 7811-7816.

Mari, M., Griffith, J., Rieter, E., Krishnappa, L., Klionsky, D. J. and Reggiori, F. (2010). An Atg9-containing compartment that functions in the early steps of autophagosome biogenesis. J Cell Biol 190, 1005-1022.

Matsushita, M., Suzuki, N. N., Obara, K., Fujioka, Y., Ohsumi, Y. and Inagaki, F. (2007). Structure of Atg5.Atg16, a complex essential for autophagy. J Biol Chem 282, 6763-6772.

Matsuura, A., Tsukada, M., Wada, Y. and Ohsumi, Y. (1997). Apg1p, a novel protein kinase required for the autophagic process in Saccharomyces cerevisiae. Gene 192, 245-250.

Meijer, W. H., der Klei, van, I. J., Veenhuis, M. and Kiel, J. A. K. W. (2007). ATG genes involved in non-selective autophagy are conserved from yeast to man, but the selective Cvt and pexophagy pathways also require organism-specific genes. Autophagy 3, 106-116.

Meiling-Wesse, K., Barth, H., Voss, C., Eskelinen, E.-L., Epple, U. D. and Thumm, M. (2004). Atg21 is required for effective recruitment of Atg8 to the preautophagosomal structure during the Cvt pathway. J Biol Chem 279, 37741-37750.

Michell, R. H. and Dove, S. K. (2009). A protein complex that regulates PtdIns(3,5)P2 levels. EMBO J 28, 86-87.

Michell, R. H., Heath, V. L., Lemmon, M. A. and Dove, S. K. (2006). Phosphatidylinositol 3,5bisphosphate: metabolism and cellular functions. Trends Biochem Sci 31, 52-63.

Mijaljica, D., Nazarko, T. Y., Brumell, J. H., Huang, W.-P., Komatsu, M., Prescott, M., Simonsen, A., Yamamoto, A., Zhang, H., Klionsky, D. J., et al. (2012). Receptor protein complexes are in control of autophagy. Autophagy $\mathbf{8}$.

Mizushima, N., Noda, T. and Ohsumi, Y. (1999). Apg16p is required for the function of the Apg12p-Apg5p conjugate in the yeast autophagy pathway. EMBO J 18, 3888-3896. 
Mizushima, N., Noda, T., Yoshimori, T., Tanaka, Y., Ishii, T., George, M. D., Klionsky, D. J., Ohsumi, M. and Ohsumi, Y. (1998). A protein conjugation system essential for autophagy. Nature 395, 395-398.

Mizushima, N., Yamamoto, A., Hatano, M., Kobayashi, Y., Kabeya, Y., Suzuki, K., Tokuhisa, T., Ohsumi, Y. and Yoshimori, T. (2001). Dissection of autophagosome formation using Apg5deficient mouse embryonic stem cells. J Cell Biol 152, 657-68.

Morales Quinones, M., Winston, J. T. and Stromhaug, P. E. (2012). Propeptide of Aminopeptidase 1 Protein Mediates Aggregation and Vesicle Formation in Cytoplasm-toVacuole Targeting Pathway. Journal of Biological Chemistry 287, 10121-10133.

Motley, A. M., Nuttall, J. M. and Hettema, E. H. (2012). Pex3-anchored Atg36 tags peroxisomes for degradation in Saccharomyces cerevisiae. EMBO J 31, 2852-2868.

Mülhardt, Cornel: Der Experimentator Molekularbiologie/Genomics; 5. Auflage, München 2006, Seite 136

Mumberg, D., Muller, R. and Funk, M. (1995). Yeast vectors for the controlled expression of heterologous proteins in different genetic backgrounds. Gene 156, 119-122.

Müller, J. and Johnsson, N. (2008). Split-ubiquitin and the split-protein sensors: chessman for the endgame. ChemBioChem 9, 2029-2038.

Nair, U., Cao, Y., Xie, Z. and Klionsky, D. J. (2010). Roles of the lipid-binding motifs of Atg18 and Atg21 in the cytoplasm to vacuole targeting pathway and autophagy. Journal of Biological Chemistry 285, 11476-11488.

Nair, U., Yen, W.-L., Mari, M., Cao, Y., Xie, Z., Baba, M., Reggiori, F. and Klionsky, D. J. (2012). A role for Atg8-PE deconjugation in autophagosome biogenesis. Autophagy 8, 780-793.

Nakatogawa, H. (2013). Two ubiquitin-like conjugation systems that mediate membrane formation during autophagy. Essays Biochem. 55, 39-50.

Nakatogawa, H., Ichimura, Y. and Ohsumi, Y. (2007). Atg8, a ubiquitin-like protein required for autophagosome formation, mediates membrane tethering and hemifusion. Cell 130, 165178.

Nakatogawa, H., Ishii, J., Asai, E. and Ohsumi, Y. (2012). Atg4 recycles inappropriately lipidated Atg8 to promote autophagosome biogenesis. Autophagy 8, 177-186.

Noda, N. N., Fujioka, Y., Hanada, T., Ohsumi, Y. and Inagaki, F. (2012). Structure of the Atg12Atg5 conjugate reveals a platform for stimulating Atg8-PE conjugation. EMBO Rep.

Noda, N. N., Kumeta, H., Nakatogawa, H., Satoo, K., Adachi, W., Ishii, J., Fujioka, Y., Ohsumi, Y. and Inagaki, F. (2008). Structural basis of target recognition by Atg8/LC3 during selective autophagy. Genes Cells 13, 1211-1218.

Noda, N. N., Ohsumi, Y. and Inagaki, F. (2009). ATG systems from the protein structural point of view. Chem. Rev. 109, 1587-1598.

Noda, N. N., Ohsumi, Y. and Inagaki, F. (2010). Atg8-family interacting motif crucial for selective autophagy. FEBS Letters 584, 1379-1385.

Obara, K. and Ohsumi, Y. (2008). Dynamics and function of PtdIns(3)P in autophagy. Autophagy 4, 952-954.

Obara, K., Noda, T., Niimi, K. and Ohsumi, Y. (2008a). Transport of phosphatidylinositol 3phosphate into the vacuole via autophagic membranes in Saccharomyces cerevisiae. Genes 
Cells 13, 537-547.

Obara, K. and Ohsumi, Y. (2011). Atg14: a key player in orchestrating autophagy. International Journal of Cell Biology 2011, 713435.

Obara, K., Sekito, T. and Ohsumi, Y. (2006). Assortment of phosphatidylinositol 3-kinase complexes--Atg14p directs association of complex I to the pre-autophagosomal structure in Saccharomyces cerevisiae. Mol Biol Cell 17, 1527-1539.

Obara, K., Sekito, T., Niimi, K. and Ohsumi, Y. (2008b). The Atg18-Atg2 complex is recruited to autophagic membranes via phosphatidylinositol 3-phosphate and exerts an essential function. J Biol Chem 283, 23972-23980.

Ohashi, Y. and Munro, S. (2010). Membrane delivery to the yeast autophagosome from the Golgi-endosomal system. Mol Biol Cell 21, 3998-4008.

Pan, X., Roberts, P., Chen, Y., Kvam, E., Shulga, N., Huang, K., Lemmon, S. and Goldfarb, D. (2000). Nucleus-vacuole junctions in Saccharomyces cerevisiae are formed through the direct interaction of Vac8p with Nvj1p. Mol Biol Cell 11, 2445-2457.

Parzych, K. R. and Klionsky, D. (2013). An overview of autophagy: Morphology, mechanism and regulation. Antioxid Redox Signal.

Pashkova, N., Gakhar, L., Winistorfer, S. C., Yu, L., Ramaswamy, S. and Piper, R. C. (2010). WD40 repeat propellers define a ubiquitin-binding domain that regulates turnover of $\mathrm{F}$ box proteins. Mol Cell 40, 433-443.

Polson, H. E. J., De Lartigue, J., Rigden, D. J., Reedijk, M., Urbé, S., Clague, M. J. and Tooze, S. A. (2010). Mammalian Atg18 (WIPI2) localizes to omegasome-anchored phagophores and positively regulates LC3 lipidation. Autophagy 6.

Proikas-Cezanne, T. and Robenek, H. (2011). Freeze-fracture replica immunolabelling reveals human WIPI-1 and WIPI-2 as membrane proteins of autophagosomes. J. Cell. Mol. Med. 15, 2007-2010.

Proikas-Cezanne, T., Waddell, S., Gaugel, A., Frickey, T., Lupas, A. and Nordheim, A. (2004). WIPI-1alpha (WIPI49), a member of the novel 7-bladed WIPI protein family, is aberrantly expressed in human cancer and is linked to starvation-induced autophagy. Oncogene 23, 9314-9325.

Ptacek, J., Devgan, G., Michaud, G., Zhu, H., Zhu, X., Fasolo, J., Guo, H., Jona, G., Breitkreutz, A., Sopko, R., et al. (2005). Global analysis of protein phosphorylation in yeast. Nature 438, 679-684.

Ragusa, M. J., Ragusa, M. J., Stanley, R. E., Stanley, R. E., Hurley, J. H. and Hurley, J. H. (2012). Architecture of the Atg17 Complex as a Scaffold for Autophagosome Biogenesis. Cell.

Reggiori, F. and Klionsky, D. J. (2013). Autophagic Processes in Yeast: Mechanism, Machinery and Regulation. Genetics 194, 341-361.

Reggiori, F., Tucker, K., Stromhaug, P. and Klionsky, D. (2004). The Atg1-Atg13 complex regulates Atg9 and Atg23 retrieval transport from the pre-autophagosomal structure. Dev Cell 6, 79-90.

Rieter, E., Vinke, F., Bakula, D., Cebollero, E., Ungermann, C., et al. (2013). Atg18 function in autophagy is regulated by specific sites within its $\beta$-propeller. J Cell Sci 126, 593-604.

Roberts, P., Moshitch-Moshkovitz, S., Kvam, E., O'Toole, E., Winey, M. and Goldfarb, D. (2003). Piecemeal microautophagy of nucleus in Saccharomyces cerevisiae. Mol Biol Cell 14, 
129-141.

Romanov, J., Walczak, M., Ibiricu, I., Schüchner, S., Ogris, E., Kraft, C. and Martens, S. (2012). Mechanism and functions of membrane binding by the Atg5-Atg12/Atg16 complex during autophagosome formation. EMBO J.

Sakoh-Nakatogawa, M., Matoba, K., Asai, E., Kirisako, H., Ishii, J., Noda, N. N., Inagaki, F., Nakatogawa, H. and Ohsumi, Y. (2013). Atg12-Atg 5 conjugate enhances E2 activity of Atg3 by rearranging its catalytic site. Nat Struct Mol Biol 20, 433-439.

Schlumpberger, M., Schaeffeler, E., Straub, M., Bredschneider, M., Wolf, D. H. and Thumm, M. (1997). AUT1, a gene essential for autophagocytosis in the yeast Saccharomyces cerevisiae. J Bacteriol 179, 1068-1076.

Schu, P., Takegawa, K., Fry, M., Stack, J., Waterfield, M. and Emr, S. (1993).

Phosphatidylinositol 3-kinase encoded by yeast VPS34 gene essential for protein sorting. Science 260, 88-91.

Scott, S., Baba, M., Ohsumi, Y. and Klionsky, D. (1997). Aminopeptidase I is targeted to the vacuole by a nonclassical vesicular mechanism. J Cell Biol 138, 37-44.

Scott, S., Guan, J., Hutchins, M., Kim, J. and Klionsky, D. (2001). Cvt19 is a receptor for the cytoplasm-to-vacuole targeting pathway. Mol Cell 7, 1131-1141.

Scott, S., Hefner-Gravink, A., Morano, K., Noda, T., Ohsumi, Y. and Klionsky, D. (1996). Cytoplasm-to-vacuole targeting and autophagy employ the same machinery to deliver proteins to the yeast vacuole. Proceed. Nat. Acad. Sci. USA 93, 12304-12308.

Scott, S., Nice, D., Nau, J., Weisman, L., Kamada, Y., Keizer-Gunnink, I., Funakoshi, T., Veenhuis, M., Ohsumi, Y. and Klionsky, D. (2000). Apg13p and Vac8p are part of a complex of phosphoproteins that are required for cytoplasm to vacuole targeting. J Biol Chem 275, 25840-25849.

Sekito, T., Kawamata, T., Ichikawa, R., Suzuki, K. and Ohsumi, Y. (2009). Atg17 recruits Atg9 to organize the pre-autophagosomal structure. Genes Cells 14, 525-538.

Sheng, Y., Mancino, V. and Birren, B. (2005). Transformation of Escherichia coli with large DNA molecules by electroporation. nucleic acids research $23,1-7$.

Shintani, T. and Klionsky, D. J. (2004). Cargo proteins facilitate the formation of transport vesicles in the cytoplasm to vacuole targeting pathway.J Biol Chem 279, 29889-29894.

Shintani, T., Huang, W., Stromhaug, P. and Klionsky, D. (2002). Mechanism of cargo selection in the cytoplasm to vacuole targeting pathway. Dev Cell 3, 825-837.

Shintani, T., Mizushima, N., Ogawa, Y., Matsuura, A., Noda, T. and Ohsumi, Y. (1999). Apg10p, a novel protein-conjugating enzyme essential for autophagy in yeast. EMBO J 18, 52345241.

Skarp, K.-P., Zhao, X., Weber, M. and Jantti, J. (2008). Use of bimolecular fluorescence complementation in yeast Saccharomyces cerevisiae. Methods Mol Biol 457, 165-175.

Stanley, R. E., Ragusa, M. J. and Hurley, J. H. (2013). The beginning of the end: how scaffolds nucleate autophagosome biogenesis. Trends Cell Biol.

Straub, M., Bredschneider, M. and Thumm, M. (1997). AUT3, a serine/threonine kinase gene, is essential for autophagocytosis in Saccharomyces cerevisiae. J Bacteriol 179, 3875-3883.

Stromhaug, P. E., Reggiori, F., Guan, J., Wang, C.-W. and Klionsky, D. J. (2004). Atg21 is a 
phosphoinositide binding protein required for efficient lipidation and localization of Atg8 during uptake of aminopeptidase I by selective autophagy. Mol Biol Cell 15, 3553-3566.

Sugawara, K., Suzuki, N., Fujioka, Y., Mizushima, N., Ohsumi, Y. and Inagaki, F. (2004). The crystal structure of microtubule-associated protein light chain 3, a mammalian homologue of Saccharomyces cerevisiae Atg8. Genes Cells 9, 611-618.

Suzuki, K. (2012). Selective autophagy in budding yeast. Cell Death Differ-.

Suzuki, K. and Ohsumi, Y. (2007). Molecular machinery of autophagosome formation in yeast, Saccharomyces cerevisiae. FEBS Letters 581, 2156-2161.

Suzuki, K. and Ohsumi, Y. (2010). Current knowledge of the pre-autophagosomal structure (PAS). FEBS Letters 584, 1280-1286.

Suzuki, K., Akioka, M., Kondo-Kakuta, C., Yamamoto, H. and Ohsumi, Y. (2013). Fine mapping of autophagy-related proteins during autophagosome formation in Saccharomyces cerevisiae. J Cell Sci 126, 2534-2544.

Suzuki, K., Kirisako, T., Kamada, Y., Mizushima, N., Noda, T. and Ohsumi, Y. (2001). The preautophagosomal structure organized by concerted functions of APG genes is essential for autophagosome formation. EMBO J 20,5971-5981.

Suzuki, K., Kubota, Y., Sekito, T. and Ohsumi, Y. (2007). Hierarchy of Atg proteins in preautophagosomal structure organization. Genes Cells 12, 209-218.

Suzuki, N. N., Yoshimoto, K., Fujioka, Y., Ohsumi, Y. and Inagaki, F. (2005). The crystal structure of plant ATG12 and its biological implication in autophagy. Autophagy 1, 119-126.

Tamura, N., Oku, M., Ito, M., Noda, N. N., Inagaki, F., et al. (2013). Atg18 phosphoregulation controls organellar dynamics by modulating its phosphoinositide-binding activity. J Cell Biol 202, 685-698.

Tanida, I., Mizushima, N., Kiyooka, M., Ohsumi, M., Ueno, T., Ohsumi, Y. and Kominami, E. (1999). Apg7p/Cvt2p: A novel protein-activating enzyme essential for autophagy. Mol Biol Cell 10, 1367-1379.

Taylor, R., Taylor, R., Chen, P.-H., Chen, P.-H., Chou, C.-C., Chou, C.-C., Patel, J., Patel, J., Jin, S. V. and Jin, S. V. (2012). KCS1 deletion in Saccharomyces cerevisiae leads to a defect in translocation of autophagic proteins and reduces autophagosome formation. Autophagy $\mathbf{8}$, 19-18.

Thumm, M., Egner, R., Koch, B., Schlumpberger, M., Straub, M., Veenhuis, M. and Wolf, D. H. (1994). Isolation of autophagocytosis mutants of Saccharomyces cerevisiae. FEBS Letters 349, 275-280.

Wach, A., Brachat, A., Alberti-Segui, C., Rebischung, C. and Philippsen, P. (1997). Heterologous HIS3 marker and GFP reporter modules for PCR-targeting in Saccharomyces cerevisiae. Yeast 13, 1065-1075.

Wach, A., Brachat, A., Pohlmann, R. and Philippsen, P. (1994). New heterologous modules for classical or PCR-based gene disruptions in Saccharomyces cerevisiae. Yeast 10, 1793-1808.

Watanabe, Y., Kobayashi, T., Yamamoto, H., Hoshida, H., Akada, R., Inagaki, F., Ohsumi, Y. and Noda, N. N. (2012). Structure-based analyses reveal distinct binding sites for Atg2 and phosphoinositides in Atg18. Journal of Biological Chemistry 287, 31681-31690.

Wittke, S., Lewke, N., Müller, S. and Johnsson, N. (1999). Probing the molecular environment of membrane proteins in vivo. Mol Biol Cell 10, 2519-2530. 
Xie, Z., Nair, U. and Klionsky, D. J. (2008). Atg8 controls phagophore expansion during autophagosome formation. Mol Biol Cell 19, 3290-3298.

Xu, C. and Min, J. (2011). Structure and function of WD40 domain proteins. Protein Cell 2, 202214.

Yamaguchi, M., Noda, N. N., Yamamoto, H., Shima, T., Kumeta, H., Kobashigawa, Y., Akada, R., Ohsumi, Y. and Inagaki, F. (2012). Structural insights into Atg10-mediated formation of the autophagy-essential Atg12-Atg5 conjugate. Structure 20, 1244-1254.

Yamamoto, H., Kakuta, S., Watanabe, T. M., Kitamura, A., Sekito, T., Kondo-Kakuta, C., Ichikawa, R., Kinjo, M. and Ohsumi, Y. (2012). Atg9 vesicles are an important membrane source during early steps of autophagosome formation. J Cell Biol 198, 219-233.

Yen, W.-L. and Klionsky, D. J. (2007). Atg27 is a second transmembrane cycling protein. Autophagy 3, 254-256.

Yen, W.-L., Legakis, J. E., Nair, U. and Klionsky, D. J. (2007). Atg27 is required for autophagydependent cycling of Atg9. Mol Biol Cell 18, 581-593.

Yorimitsu, T. and Klionsky, D. (2005). Autophagy: molecular machinery for self-eating. Cell Death Differ 12 Suppl 2, 1542-1552.

Yorimitsu, T., He, C., Wang, K. and Klionsky, D. J. (2009). Tap42-associated protein phosphatase type 2A negatively regulates induction of autophagy. Autophagy 5, 616-624.

Yu, Z.-Q., Ni, T., Hong, B., Wang, H.-Y., Jiang, F.-J., Zou, S., Chen, Y., Zheng, X.-L., Klionsky, D. J., Liang, Y., et al. (2012). Dual roles of Atg8-PE deconjugation by Atg 4 in autophagy. Autophagy 8, 877-876.

Yuga, M., Gomi, K., Klionsky, D. J. and Shintani, T. (2011). Aspartyl aminopeptidase is imported from the cytoplasm to the vacuole by selective autophagy in Saccharomyces cerevisiae. Journal of Biological Chemistry 286, 13704-13713. 


\section{Acknowledgement}

Ganz besonders möchte ich Herrn Prof. Michael Thumm für die Bereitstellung dieses interessanten Themas, für die Möglichkeit zur Promotion und für die Unterstützung in sämtlichen Bereichen danken!

Prof. Volka Lipka möchte ich für die Übernahme des Zweitgutachtens danken!

Vielen Dank an alle Mitgliedern meines Thesis Committees: Herrn Prof. Thumm, Herrn Prof. Lipka und Frau Dr. Kühnel für alle wissenschaftlichen Ratschläge und wertvolle Anregungen!

Des Weiteren danke ich der Göttinger Graduate School for Neuroscience, Biophysics and Molecular Biosciences (DFG Grants GSC 226/1, GSC 226/2) für die finanzielle Unterstützung während der gesamten Promotionszeit!

Ein besonderer Dank geht an Rosi, die mir jederzeit unterstützend zur Seite stand. Vielen Dank für die vielen hilfreichen Ratschläge und dein offenes Ohr zu jeder Zeit!

Weiterhin möchte ich Klaus Neiffer für die geduldige Durchführung der zahlreichen Gelfiltrationen danken!

Ganz besonders möchte ich auch der gesamten Arbeitsgruppe Thumm: Petra, Rosi, Peter, Marco und auch der Ehemaligen Evelyn für das humorvolle Laborklima, die vielen netten Gespräche in- und außerhalb des Labors und die tolle Zeit bedanken!

Mein großer Dank geht an meinen Freund Benjamin Voß sowie an meine Familie und Freunde, die jederzeit an mich geglaubt haben und mich stets kräftig unterstützt haben!

Vielen Dank! 